\title{
On Infravacua and the Superselection Structure of Theories with Massless Particles
}

\author{
Dissertation \\ zur Erlangung des Doktorgrades \\ der Mathematisch-Naturwissenschaftlichen Fakultäten \\ der Georg-August-Universität zu Göttingen
}

vorgelegt von

Walter Kunhardt

aus Berchem-Ste.-Agathe

(Belgien)

Göttingen 2001 
D 7

Referent: Prof. Dr. D. Buchholz

Korreferent: Prof. Dr. H. Roos

Tag der mündlichen Prüfung: 27/06/2001 


\section{Contents}

1 Introduction 5

1.1 The Physical Context . . . . . . . . . . . . . . . . . . . . 5

I.2 The Mathematical Framework . . . . . . . . . . . . . . . 7

L.3 Structure of This Thesis . . . . . . . . . . . . . . . . . 9

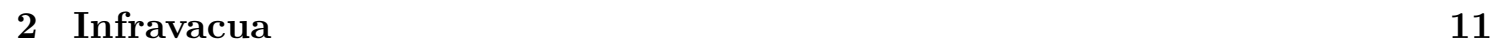

2.1 Energy Components . . . . . . . . . . . . . . . . . . . . . 11

2.2 Infravacuum Representations $\ldots \ldots \ldots \ldots \ldots$

3 Superselection Theory with General Localisation 17

3.1 Assumptions and Basic Properties . . . . . . . . . . . . . . . 18

3.2 Connectedness and Enlargement Properties of $\mathcal{X}] \ldots \ldots \ldots$

3.3 Composition of Sectors . . . . . . . . . . . . . . . . . . . 24

3.4 The Symmetry . . . . . . . . . . . . . . . . . . . . . . . . . . . . . . . . . . . . 28

3.5 Conjugates and Left Inverses $\ldots \ldots \ldots \ldots \ldots \ldots$

3.6 The Action of a Symmetry Group . . . . . . . . . . . . . . . . . . . . . . . . . . . . . . . 32

3.7 Charge Transporting Cocycles . . . . . . . . . . . . . . . . . . 34

\begin{tabular}{llll}
\hline & Spectral Properties & 43
\end{tabular}

4.1 Cocycles and Their Spectra . . . . . . . . . . . . . . . . . . . . . 43

4.2 Spectra of Pointlike Charges . . . . . . . . . . . . . . . . . . . . 46

4.3 The Role of the Vacuum Vector . . . . . . . . . . . . . . . . . . . 47

4.4 The Minimal Cocycles under Conjugation . . . . . . . . . . . . . . . . . 49

5 An Example for Background-Induced Localisation 53

5.1 The Model . . . . . . . . . . . . . . . . . . 54

5.2 Poor Localisation in Front of the Vacuum . . . . . . . . . . 56

5.3 Infravacuum Background States . . . . . . . . . . . . . . . . . . 57

5.4 Better Localisation in Front of KPR-like_nfravacua . . . . . . . . . . . 63

6 Conclusions and Outlook $\quad 69$

\begin{tabular}{ll}
\hline A Some Category Theory & 71
\end{tabular}

A.1 $\mathrm{C}^{*}-\mathrm{W}^{*}$ - and Monoidal Categories . . . . . . . . . . . . . . . . . 71

A.2 Conjugates, Left Inverses and Symmetry . . . . . . . . . . . . . . . . 75

A.3 Finite Statistics and the Existence of Conjugates . . . . . . . . . . . . . . 78

A.4 Standard Solutions and the Conjugation on Morphisms . . . . . . . . . . 80 
\begin{tabular}{lr}
\hline B A Homotopy Argument & 87
\end{tabular}

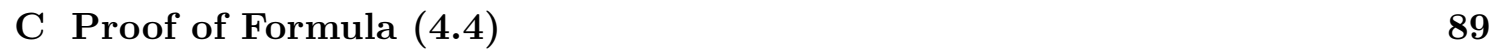

D On Distributions Which Vanish in a Wedge Region 93

D.1 The JLD Correspondence . . . . . . . . . . . . . . . . . . . . . 93

D.2 Criteria Making $f$ Vanish $\ldots \ldots \ldots \ldots \ldots$. . . . . . . . . . . . 97

D.3 Further Generalisations . . . . . . . . . . . . . . . . . . . . . . . . . . 99

$\begin{array}{ll}\text { Bibliography } & 103\end{array}$

$\begin{array}{ll}\text { Acknowledgements } & 107\end{array}$

$\begin{array}{ll}\text { Curriculum Vitae } & 109\end{array}$ 


\section{Chapter 1}

\section{Introduction}

\subsection{The Physical Context}

The known phenomena of high energy physics are described very successfully on the theoretical side by relativistic quantum field theories. On a formal level these theories arise by "quantisation" from a classical field theory, regarded as a mechanical system with infinitely many degrees of freedom. Introduced briefly after the invention of quantum mechanics itself, quantum electrodynamics (QED) is one of the oldest quantum field theories; it describes the interaction between electrons, positrons and photons, the quanta of the electromagnetic field. Technically, QED is a gauge theory, and as such it is the prototype of essential parts of the Standard Model of particle physics.

The great degree of precision to which QED (or rather, the Standard Model) is in agreement with experiments may be illustrated by the recent controversy about a $5 \mathrm{ppm}$ mismatch between theory and experiment regarding the anomaly of the magnetic moment of the muon [I]. It must however be contrasted with the mathematical difficulties connected with its very definition. As examples, let us just mention the (perturbative) treatment of interactions and the presence of unobservable gauge fields.

It has been clear for about forty years that the mathematical problems connected with quantum field theories can best be analysed in a model-independent setting. There exist several approaches in this spirit, all believed to be essentially equivalent. The oldest and the one most closely related to the recipe of quantising a classical field theory is Wightman's approach in terms of operator-valued distributions [2]; among its important successes are the PCT-theorem and the derivation of the spin-statistics connection. A bit more abstract is the algebraic approach of Haag and Kastler [3], which is also used in the subsequent work and therefore introduced in more detail in Section 1.2. Based on the algebra of local observables of the model under consideration, it does not presuppose the existence of unobservable (gauge) fields, and it is thus all the more remarkable that the structure of the charged states appearing in such a model (when applied to particle physics) can be shown to match that of a gauge theory. Beyond superselection theory (or DHR theory, after its inventors Doplicher, Haag and Roberts), as this area is called, the algebraic formulation of quantum field theory is also useful for the investigation of other kinds of questions such as renormalisation, the analysis of thermal states or quantum field theory on curved space-time. As these aspects are of no direct interest in the sequel, we refer the reader to the monograph [4].

Although concepts known form gauge theories have a natural place in algebraic quan- 
tum field theory, problems appear when it comes to QED, which at first sight might even seem to be among the simplest gauge theories. The problems are due to the fact that photons are massless particles (and in the present understanding of the Standard Model they are likely to be the only massless particles), or, put differently, that QED is governed by a long-range interaction. In field-theoretical terms, this manifests itself in Gauss' law: each electrically charged particle is accompanied by an electromagnetic field with $1 / r^{2}$ asymptotic behaviour in spacelike directions, and the total electric flux through an arbitrarily large sphere equals the charge of the particle.

For the mathematical formulation of the associated quantum theory, this means that an operator $\psi$ which creates in the underlying Hilbert space an electrically charged state from the vacuum $\Omega_{0}$, cannot be local. Since it must account for the electric field as well, this operator may at best be localised in a region which extends to spacelike infinity such as, e.g., a spacelike cone $\mathcal{C}$. In the causal complement $\mathcal{C}^{\prime}$ of $\mathcal{C}$, the state $\psi \Omega_{0}$ then is indistinguishable from the vacuum.

Now whereas the charge can be localised in this very restrictive way, another essential property necessary for superselection theory to be applicable still fails: the charge is not transportable in the sense that given some other spacelike cone $\mathcal{C}_{1}$, there is no vector $A \psi \Omega_{0}$ which belongs to the same superselection sector as $\psi \Omega_{0}$ (i.e., which can be prepared from the state $\psi \Omega_{0}$ by a local operation $A$ ) and which describes the same charge but with its electromagnetic field concentrated in $\mathcal{C}_{1}$. This is due to Einstein causality, by which any local observable $A$ must commute with the observable (formally given by $\mathcal{E}_{\text {as }}(\vec{n})=\lim _{r \rightarrow \infty} r^{2} \vec{n} \overrightarrow{\mathcal{E}}(r \vec{n})$ ) which describes the asymptotic form of the electric field $\overrightarrow{\mathcal{E}}$ in the direction $\vec{n}$. In other words, $\mathcal{E}_{\text {as }}(\vec{n})$ is a classical observable, and the superselection sector of $\psi \Omega_{0}$ is characterised by the asymptotic electric field rather than just its integral over the unit sphere.

In the above heuristic argument, it has tacitly been supposed that the asymptotic value of the electric field is well-defined in the states under consideration. While this is certainly true for the vacuum (and for states describing e.g. a single charged particle in uniform motion), it is unlikely to be the case for physically more realistic states. Collision processes between charged particles for instance are inevitably accompanied by the emission of bremsstrahlung. In the particle picture, this amounts to the creation of infinitely many photons. That this does not conflict with energy conservation is of course again due to the masslessness of the photon.

The mathematical counterpart of the creation of infinitely many photons is the fact that the representations of the incoming and the outgoing free electromagnetic fields in such a process cannot both be Fock representations. But there is still the possibility that these two representations are equivalent, namely if they are sufficiently chaotic so as to be stable under the addition of the bremsstrahlung photons. With a reasonable model of the latter, such representations of the free electromagnetic field have actually been constructed by Kraus, Polley and Reents [5].

Motivated by this picture, Buchholz has proposed in [6] that a possible way of improving the localisation properties of the electric charges (so as to make the analysis of superselection sectors available to an algebraic formulation of QED) should be to drop the over-idealisation inherent in describing the charges in front of the vacuum. If instead the charges are viewed together with a background with sufficiently high fluctuations, then the value of the asymptotic electric field will cease to be a meaningful quantity as far as "individual" directions (even after smearing) are concerned, while the total asymptotic 
electric flux still is well-defined. The mechanism behind this is that the statistical error in the measurement of $\mathcal{E}_{\text {as }}(\vec{n})$ diverges (in the limit $r \rightarrow \infty$ ) while through the long-range correlations this error vanishes in a measurement of $\int_{S^{2}} d \Omega(\vec{n}) \mathcal{E}_{\text {as }}(\vec{n})$. In particular, Gauss' law still holds in these states.

We will use the generic term "infravacua" to designate background states or the corresponding representations which comply with the intuitive idea of differing from the vacuum by some ("infrared") cloud of infinitely many massless particles leading to the above-mentioned fluctuations. The central task is of course to give a mathematical characterisation of such states and to show that their properties are indeed in accordance with the heuristic picture. A typical, yet very special class of such states should be provided in all theories with massless particles by the so-called KPR-states constructed along the lines of $[5]$.

In the following work, we will give a tentative definition of infravacuum states and derive some of their general properties. As a characteristic feature of infravacua, the associated spectrum of the energy-momentum operator is the entire forward light cone $\overline{\mathbf{V}}_{+}$, but is does not - in distinction to the vacuum — contain $\{0\}$ as a discrete point. We will then review the DHR theory of superselection sectors in a formulation suited to charges localisable in a restricted sense like the above-mentioned one in front of an infravacuum background. The algebraic aspects of this theory do not depend on the presence of a translation invariant vector, but when it comes to the analysis of energy-momentum spectra in the charged sectors, the traditional treatments $[\bar{z}, \underline{8}, \underline{9}]$ use the translation invariance of the vacuum at some technically crucial points. The most prominent example is perhaps the proof that a particle and its antiparticle have equal masses. Now this corresponds to an experimentally well-established fact, and it has certainly not been established in an environment totally void of infrared radiation. If infravacua are viable substitutes for the vacuum it should therefore be possible to derive the same spectral properties without relying on the existence of a translation invariant vector. We will investigate to what an extent this is actually possible and obtain an encouraging partial result in this direction.

Moreover we will see in a simple model of a free field that Buchholz' idea of improving the localisation properties of charges by using an infravacuum background is indeed successful.

The results of this thesis therefore support the more general belief that infravacua provide the basis for a realistic description of theories with massless particles. A more thorough verification of this hypothesis would of course also comprise an analysis of how numerical or structural results (such as e.g. masses of particles or the superselection structure) depend on the chosen infravacuum background. We will however not touch upon such questions here.

\subsection{The Mathematical Framework}

The present work is based on the algebraic approach to relativistic quantum field theory [3]. Generally, a system in quantum physics is described by a non-commutative unital $\mathrm{C}^{*}$-algebra $\mathfrak{A}$, the self-adjoint elements of which are interpreted as (idealised) observables. A state of that system is then given by a normalised positive linear functional

$$
\omega: \mathfrak{A} \longrightarrow \mathbb{C}
$$


which assigns to each observable $a \in \mathfrak{A}$ the number $\omega(a)$ interpreted as its expectation value.?

This abstract viewpoint is particularly useful in quantum field theory [10]. The systems considered there possess infinitely many degrees of freedom, and the algebra $\mathfrak{A}$ of observables therefore has infinitely many inequivalent irreducible Hilbert space representations. In distinction to quantum mechanics, it is thus not sufficient to restrict the attention to the normal states in one fixed (irreducible) representation only. Rather, the characterisation of physically meaningful representations becomes a nontrivial issue.

Relativistic quantum field theory has special relativity as another main ingredient. An essential feature of relativity is Einstein causality: physical effects cannot propagate faster than the speed of light. Measurements effectuated in two causally disjoint regions of spacetime therefore do not influence each other. By the principles of quantum physics this means that the operators describing such a pair of measurements must commute. As a consequence of this (and of the elementary observation that each measurement is localised somewhere in spacetime), the $\mathrm{C}^{*}$-algebra of observables describing in the algebraic framework a quantum field theory in $(1+s)$-dimensional Minkowski spacetime has a very rich structure. It is usually defined in its vacuum representation. Mathematically, this amounts to the following assumptions:

Let $\left(\mathcal{H}_{0}, U_{0}, \Omega_{0}\right)$ be a vacuum Hilbert space, that is a Hilbert space $\mathcal{H}_{0}$ which carries a strongly continuous unitary representation $U_{0}$ of the spacetime translation group $\mathbb{R}^{1+s}$ whose spectrum is contained in the forward light cone $\overline{\mathbf{V}}_{+}:=\left\{p \in \mathbb{R}^{1+s} \mid p^{2} \geq 0\right\}$ and which is such that the $U_{0}$-invariant subspace of $\mathcal{H}_{0}$ is spanned by a single unit vector $\Omega_{0}$, called the vacuum vector. The physical system is described by a Haag-Kastler net

$$
\mathcal{O} \longmapsto \mathfrak{A}(\mathcal{O}) \subset \mathcal{B}\left(\mathcal{H}_{0}\right)
$$

of von Neumann algebras on $\mathcal{H}_{0}$, i.e., a mapping which assigns to each bounded open set $\mathcal{O} \subset \mathbb{R}^{1+s}$ a von Neumann algebra whose self-adjoint elements are interpreted as quantities observable in the region $\mathcal{O}$. This net has to have the following properties:

$$
\begin{array}{rlll}
\mathfrak{A}\left(\mathcal{O}_{1}\right) \subset \mathfrak{A}\left(\mathcal{O}_{2}\right) & \text { if } & \mathcal{O}_{1} \subset \mathcal{O}_{2} & \text { (isotony), } \\
{\left[\mathfrak{A}\left(\mathcal{O}_{1}\right), \mathfrak{A}\left(\mathcal{O}_{2}\right)\right]=\{0\}} & \text { if } & \mathcal{O}_{1} \times \mathcal{O}_{2} & \text { (locality), } \\
\alpha_{x}^{0}(\mathfrak{A}(\mathcal{O}))=\mathfrak{A}(\mathcal{O}+x) & \text { if } & x \in \mathbb{R}^{1+s} & \text { (covariance). }
\end{array}
$$

Here $\alpha_{x}^{0}:=\operatorname{Ad} U_{0}(x)$ denotes the adjoint action of the translation group and $\mathbf{X}$ denotes the causal disjointness relation defined between arbitrary subsets $X_{j} \subset \mathbb{R}^{1+s}(j=1,2)$ by

$$
X_{1} \times X_{2} \quad: \Longleftrightarrow\left(x_{1}-x_{2}\right)^{2}<0 \text { for all } x_{1} \in X_{1}, x_{2} \in X_{2} .
$$

An unbounded region $R \subset \mathbb{R}^{1+s}$ gets associated the concrete $\mathrm{C}^{*}$-algebra

$$
\mathfrak{A}(R):=\overline{\mathcal{O} \subset R}_{\bigcup \mathfrak{A}(\mathcal{O})}{ }^{\|\cdot\|},
$$

\footnotetext{
${ }^{1}$ The contact to the more usual formulation on a Hilbert space is made via the Gelfand-NaimarkSegal (GNS) reconstruction theorem. It asserts that there is a (up to unitary equivalence, unique) triple $\left(\mathcal{H}_{\omega}, \pi_{\omega}, \Omega_{\omega}\right)$ consisting of a Hilbert space $\mathcal{H}_{\omega}$, a (unital $\left.{ }_{-}\right)$representation $\pi_{\omega}: \mathfrak{A} \longrightarrow \mathcal{B}\left(\mathcal{H}_{\omega}\right)$ and a cyclic vector $\Omega_{\omega} \in \mathcal{H}_{\omega}$ such that $\omega(a)=\left\langle\Omega_{\omega}, \pi_{\omega}(a) \Omega_{\omega}\right\rangle$ for all $a \in \mathfrak{A}$.
} 
and we denote with $\mathfrak{A}_{0}$ the quasilocal algebra of this net, i.e., the inductive limit

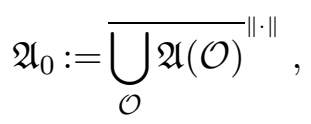

and assume that it acts irreducibly on $\mathcal{H}_{0}$, i.e.,

$$
\mathfrak{A}_{0}^{\prime}=\mathbb{C} \mathbf{1}_{\mathcal{H}_{0}} .
$$

(For any set $B$ of bounded operators on some Hilbert space, $B^{\prime}$ denotes its commutant, that is the set of all bounded linear operators which commute with each element of $B$.)

The last structural assumption to be listed here is called weak additivity and encodes the idea that there is some field theory which underlies the given net $\mathfrak{A}$ : for each open set $\mathcal{O}$, one has

$$
\mathfrak{A}_{0}^{\prime \prime}=\left(\bigcup_{x \in \mathbb{R}^{1+s}} \mathfrak{A}(\mathcal{O}+x)\right)^{\prime \prime} .
$$

By the arguments collected nicely in [II] it follows from these assumptions that $\mathfrak{A}_{0}$ is a simple $\mathrm{C}^{*}$-algebra, i.e., that is has no nontrivial *-ideals; in particular, every representation of $\mathfrak{A}_{0}$ is faithful.

In contrast to more specific additional assumption made only in certain parts, the above assumptions shall be valid throughout all of the following work.

For later use, let us introduce here some more terminology and notations: The identical representation $\pi_{0}: \mathfrak{A}_{0} \longrightarrow \mathcal{B}\left(\mathcal{H}_{0}\right)$ is also called the vacuum representation since it is the GNS representation of the vacuum state $\omega_{0}: \mathfrak{A}_{0} \longrightarrow \mathbb{C}: a \longmapsto \omega_{0}(a)=\left\langle\Omega_{0}, a \Omega_{0}\right\rangle$. The spectral family associated with the translations $U_{0}$ is denoted by $E_{0}$.

A very important criterion which selects other representations of interest for particle physics at zero temperature is what is called positivity of the energy: A representation $\pi: \mathfrak{A}_{0} \longrightarrow \mathcal{B}(\mathcal{H})$ of the quasilocal algebra on some Hilbert space $\mathcal{H}$ is said to have positive energy if there exists a strongly continuous unitary representation $U: \mathbb{R}^{1+s} \longrightarrow \mathcal{B}(\mathcal{H})$ of the translation group satisfying

$$
\pi \circ \alpha_{x}^{0}=\operatorname{Ad} U(x) \circ \pi \quad \text { and } \quad \operatorname{sp} U \subset \overline{\mathbf{V}}_{+} .
$$

In this situation, it is known [12] that the representation $U$ can be chosen such that $U(x) \in \pi\left(\mathfrak{A}_{0}\right)^{\prime \prime}$, and moreover [[13] that there is a unique such choice for which, on any subspace $\mathcal{H}^{\prime} \subset \mathcal{H}$ invariant under $\pi\left(\mathfrak{A}_{0}\right)$, the spectrum $\left.\operatorname{sp} U\right|_{\mathcal{H}^{\prime}}$ has a Lorentz-invariant lower boundary. This choice is called the minimal representation of the translations for the representation $(\mathcal{H}, \pi)$, and we will reserve for it the notation $U_{\pi}$ (and, correspondingly, for its spectral family the notation $E_{\pi}$ ). It has been shown in [14] that $\left.\operatorname{sp} U\right|_{\mathcal{H}^{\prime}}$ is actually a Lorentz-invariant subset of $\overline{\mathbf{V}}_{+}$. The monograph [15] is a standard reference for a thorough discussion of these and related topics.

\subsection{Structure of This Thesis}

The structure of this thesis is as follows. In Chapter 2 a notion of infravacuum representations is presented and some properties of such representations are described. Chapter 3 contains an account of the DHR theory of superselection sectors for charges with general 
localisation properties. We pay particular attention to the categorical aspects of the theory and to the description of covariant objects by means of charge transporting cocycles. In Chapter 4 we investigate the properties of energy-momentum spectra associated to translation covariant charges in front of an infravacuum background. We make a step towards showing that the properties of the spectra known in front of the vacuum continue to hold in the infravacuum case as well. In Chapter 5 we discuss in the example of the free massless scalar field a class of charges which become much better localised in front of a suitable infravacuum background than in front of the vacuum. Chapter 6, finally, is devoted to conclusions and perspectives.

The appendices discuss several mathematical topics. Appendix $\mathbf{A}$ represents the mathematical background of Chapter 3; we collect numerous definitions and notions in the context of monoidal $\mathrm{C}^{*}$-categories. It is intended to be a reasonably self-contained and pedagogical overview. In Appendix B we prove a technical lemma in connection with cocycles used at several places in Section 3.7. In Appendix $\mathbf{C}$ we give the proof in categorical terms of a formula crucial to Section 4.4. Appendix $\mathbf{D}$ too is related to Chapter 田, since it contains the rigorous derivation of an argument necessary there for connecting position and momentum space properties of distributions.

The content of Chapter 2 and parts of Chapter 4 represent the content of [16], whereas the example treated in Chapter 5 has been published in [IT]. 


\section{Chapter 2}

\section{Infravacua}

In this chapter we will introduce the notion of infravacuum representations of the algebra $\mathfrak{A}_{0}$. The idea behind this notion is that a vector state in such a representation should describe a physical situation where some infrared cloud but no total charge is present. Originally, infrared clouds appear in quantum electrodynamics and consist (for any energy threshold $\epsilon>0$ ) of a finite number $N_{\epsilon}$ of "hard" photons and an infinite number of "soft" photons with total energy less than $\epsilon$. They can thus be regarded as a certain finite energy limits (outside the vacuum sector) of states which do belong to the vacuum sector. The definition of infravacuum representations presented below tries to translate this characteristic feature into the model-independent framework. It is based upon the notion of energy components of positive energy representations.

\subsection{Energy Components}

Energy components are certain sets of states associated to positive energy representations of $\mathfrak{A}_{0}$. They have been introduced by Borchers and Buchholz [1.3] and discussed further by Wanzenberg [18]. This last reference being difficultly accessible, we repeat some of its results here.

Let $(\mathcal{H}, \pi)$ be a positive energy representation of $\mathfrak{A}_{0}$ and $E_{\pi}$ the spectral family of the associated minimal representation $U_{\pi}$ of the translations (cf. Section 1.2). The naturality of $U_{\pi}$ implies that there is a natural notion of energy contents of $\pi$-normal states: If $D \subset \overline{\mathbf{V}}_{+}$is a compact subset of the forward light cone,, $]$denote by

$$
\mathcal{S}_{\pi}(D):=\left\{\operatorname{tr} \rho \pi(\cdot) \mid \rho \in \mathcal{I}_{1}(\mathcal{H}), \rho \geq 0, \operatorname{tr} \rho=1, \rho E_{\pi}(D)=\rho\right\}
$$

the set of all $\pi$-normal states which have energy-momentum in $D$. Then $\mathcal{S}_{\pi}=\overline{\bigcup_{D} \mathcal{S}_{\pi}(D)}\|\cdot\|$ is the folium of $\pi$. A larger set $\tilde{\mathcal{S}}_{\pi}$ of states, called the energy component of $\pi$, is defined by

$$
\tilde{\mathcal{S}}_{\pi}:={\overline{\bigcup_{D} \tilde{\mathcal{S}}_{\pi}(D)}}^{\|\cdot\|},
$$

where $\tilde{\mathcal{S}}_{\pi}(D)$ stands for the set of all locally $\pi$-normal states in the weak closure of $\mathcal{S}_{\pi}(D)$. It can be shown that, like $\mathcal{S}_{\pi}$, also $\tilde{\mathcal{S}}_{\pi}$ is a folium, that is, a closed convex subset of the

\footnotetext{
${ }^{1}$ In this chapter, $D$ will always stand for a compact subset of $\overline{\mathbf{V}}_{+}$. Moreover we will use the notation $D_{q}:=\overline{\mathbf{V}}_{+} \cap\left(q+\overline{\mathbf{V}}_{-}\right)$for the double cone in momentum space with apices 0 and $q \in \mathbf{V}_{+}$.
} 
set of all states over $\mathfrak{A}_{0}$ stable under the operations $\omega \longmapsto \omega_{A}:=\omega\left(A^{*} \cdot A\right), A \in \mathfrak{A}_{0}$ such that $\omega\left(A^{*} A\right)=1$. Physically, $\tilde{\mathcal{S}}_{\pi}$ is interpreted as the set of states which can be reached from states in $\mathcal{S}_{\pi}$ by operations requiring only a finite amount of energy. Some important properties of the elements of $\tilde{\mathcal{S}}_{\pi}$ are collected in the following lemma, Parts ii and iii of which are due to Wanzenberg [18].

Lemma 2.1 Let $\omega \in \tilde{\mathcal{S}}_{\pi}$ and denote by $\left(\mathcal{H}_{\omega}, \pi_{\omega}, \Omega_{\omega}\right)$ its GNS triple. Then:

$i$. $\left(\mathcal{H}_{\omega}, \pi_{\omega}\right)$ is a locally $\pi$-normal positive energy representation of $\mathfrak{A}_{0}$.

ii. Moreover, if $\omega \in \tilde{\mathcal{S}}_{\pi}\left(D_{q}\right)$ for some $q \in \mathbf{V}_{+}$, then one has $\Omega_{\omega} \in E_{\pi_{\omega}}\left(D_{q}\right) \mathcal{H}_{\omega}$.

iii. In the situation of $i$, one has $\tilde{\mathcal{S}}_{\pi_{\omega}}(D) \subset \tilde{\mathcal{S}}_{\pi}\left(D+D_{q}-D_{q}\right)$ for any $D$.

Proof: i. Using the positivity of the energy in the representation $\pi$, Part i follows from the fact that $\omega$ is locally $\pi$-normal by arguments similar to those in [II.]. (In [I.9], these arguments are only applied to states $\omega \in \tilde{\mathcal{S}}_{\pi}\left(D_{q}\right)$, but they carry over to norm limits of such states as well.)

ii. For simplicity, Part ii will only be proved in the case where $\pi_{\omega}$ is factorial. For the general case, see [18]. Let $D_{q}$ be given. Then the idea is to show that $\Omega_{\omega}$ does not have momentum outside $D_{q}$. To this end, fix some $p \in \overline{\mathbf{V}}_{+} \backslash D_{q}$ and choose a neighbourhood $\mathcal{N}_{p} \subset \overline{\mathbf{V}}_{+} \backslash D_{q}$ of $p$ and an open set $\mathcal{N} \subset \operatorname{sp} U_{\pi_{\omega}}$ such that $\left(D_{q}+\mathcal{N}-\mathcal{N}_{p}\right) \cap \overline{\mathbf{V}}_{+}=\emptyset$. (Such a choice is always possible because $\operatorname{sp} U_{\pi_{\omega}}$ is Lorentz invariant.) Now choose a test function $f$ satisfying supp $\tilde{f} \subset \mathcal{N}-\mathcal{N}_{p}$ and take an arbitrary $A \in \mathfrak{A}_{0}$. Then $A(f):=\int d x f(x) \alpha_{x}(A)$ is an element of $\mathfrak{A}_{0}$ and satisfies $\pi(A(f)) E_{\pi}\left(D_{q}\right) \mathcal{H}_{\pi}=\{0\}$. Since $\omega \in \tilde{\mathcal{S}}_{\pi}\left(D_{q}\right)$, this implies $\omega\left(A(f)^{*} A(f)\right)=0$, hence $\pi_{\omega}(A(f)) \Omega_{\omega}=0$. This means that $\Omega_{\omega}$ is orthogonal to

$$
\mathcal{D}:=\operatorname{span}\left\{\pi_{\omega}(A(f))^{*} \Psi \mid \Psi \in \mathcal{H}_{\omega}, A \in \mathfrak{A}_{0}, \operatorname{supp} \tilde{f} \subset \mathcal{N}-\mathcal{N}_{p}\right\} .
$$

Since $\pi_{\omega}$ is factorial and $\mathcal{N}-\mathcal{N}_{p}$ is open, it follows by an argument explained in [9] (see, in particular, the proof of Prop. 2.2 therein) that the closure of $\mathcal{D}$ equals $E_{\pi_{\omega}}\left(\operatorname{sp} U_{\pi_{\omega}}+\right.$ $\left.\mathcal{N}_{p}-\mathcal{N}\right) \mathcal{H}_{\omega}$. Thus, $\Omega_{\omega} \in \mathcal{D}^{\perp}$ yields

$$
\left\{\Omega_{\omega}\right\}^{\perp} \supset \mathcal{D}^{\perp \perp}=\overline{\mathcal{D}}=E_{\pi_{\omega}}\left(\operatorname{sp} U_{\pi_{\omega}}+\mathcal{N}_{p}-\mathcal{N}\right) \mathcal{H}_{\omega} \supset E_{\pi_{\omega}}\left(\mathcal{N}_{p}\right) \mathcal{H}_{\omega}
$$

where the last inclusion holds because $\operatorname{sp} U_{\pi_{\omega}}-\mathcal{N} \ni 0$. From this, we get $E_{\pi_{\omega}}\left(\mathcal{N}_{p}\right) \Omega_{\omega}=0$ or, as $p \in \overline{\mathbf{V}}_{+} \backslash D_{q}$ was arbitrary, $\Omega_{\omega} \in E_{\pi_{\omega}}\left(D_{q}\right) \mathcal{H}_{\omega}$.

iii. To prove the last part, let $\Psi \in E_{\pi_{\omega}}(D) \mathcal{H}_{\omega}$. Part ii and the cyclicity of $\Omega_{\omega}$ imply that there exists in $\mathfrak{A}_{0}$ a sequence $\left(A_{n}\right)_{n \in \mathbb{N}}$ of operators with energy-momentum support in $D-D_{q}$ and normalised to $\omega\left(A_{n}^{*} A_{n}\right)=1$ such that $\Psi=\lim _{n \rightarrow \infty} \pi_{\omega}\left(A_{n}\right) \Omega_{\omega}$. From $\omega \in \tilde{\mathcal{S}}_{\pi}\left(D_{q}\right)$ it follows $\omega\left(A_{n}^{*} \cdot A_{n}\right) \in \tilde{\mathcal{S}}_{\pi}\left(D_{q}+\left(D-D_{q}\right)\right)=\tilde{\mathcal{S}}_{\pi}\left(D+D_{q}-D_{q}\right)$, i.e.

$$
\left\langle\Psi, \pi_{\omega}(\cdot) \Psi\right\rangle=\lim _{n \rightarrow \infty} \omega\left(A_{n}^{*} \cdot A_{n}\right) \in \tilde{\mathcal{S}}_{\pi}\left(D+D_{q}-D_{q}\right) .
$$

Thus any vector state from $\mathcal{S}_{\pi_{\omega}}(D)$ lies in $\tilde{\mathcal{S}}_{\pi}\left(D+D_{q}-D_{q}\right)$. One now gets the assertion by taking convex combinations, norm limits and locally normal weak limits.

Lemma 2.1 has shown that positivity of the energy is a property which "survives" the process of going from the representation $\pi$ to the GNS representation of a state in $\tilde{\mathcal{S}}_{\pi}$. Other properties survive as well, as for instance the compactness condition $\mathrm{C}_{\sharp}$ of Fredenhagen and Hertel [20, 21] which can be formulated as follows: 
Definition: Condition $\mathrm{C}_{\sharp}$ is said to be satisfied in the (positive energy) representation $\pi$ if, for any $D$ and any bounded region $\mathcal{O} \subset \mathbb{R}^{1+s}$, the set

$$
\left.\mathcal{S}_{\pi}(D)\right|_{\mathfrak{A}(\mathcal{O})} \equiv\left\{\left.\omega\right|_{\mathfrak{A}(\mathcal{O})} \mid \omega \in \mathcal{S}_{\pi}(D)\right\}
$$

is contained in a $\|\cdot\|$-compact subset of $\mathfrak{A}(\mathcal{O})^{*}$.

This condition controls the infrared properties of the model under consideration, cf. [21]. It has been established for the theory of a massive free particle (in any space-time dimension) and for the theory of a massless (scalar or vector) particle in at least $1+3$ space-time dimensions [22, 21] and is believed to hold in QED as well. In the present context, it will play a technical role in the proof of Prop. 2.4 since it allows (by Part i of the next lemma) a simplification in the definition of $\tilde{\mathcal{S}}_{\pi}(D)$.

Lemma 2.2 Let $\mathrm{C}_{\sharp}$ be satisfied in the representation $\pi$ and let $\omega$ be a state in the weak closure of $\mathcal{S}_{\pi}(D)$. Then:

$i$. The state $\omega$ is locally $\pi$-normal, i.e., $\omega \in \tilde{\mathcal{S}}_{\pi}(D)$.

ii. Condition $\mathrm{C}_{\sharp}$ is satisfied in the GNS representation of $\omega$.

Proof: Both parts follow from the fact that, in restriction to $\mathfrak{A}(\mathcal{O})$, any weak limit point of $\mathcal{S}_{\pi}(D)$ is, as a consequence of $\mathrm{C}_{\sharp}$, even a $\|\cdot\|$-limit point of $\mathcal{S}_{\pi}(D)$. Part i now follows

directly. For Part ii, we note that the above fact implies $\left.\tilde{\mathcal{S}}_{\pi}(D)\right|_{\mathfrak{A}(\mathcal{O})} \subset \overline{\left.\mathcal{S}_{\pi}(D)\right|_{\mathfrak{A}(\mathcal{O})}\|\cdot\|}$, which in view of Lemma 2.1, iii yields the assertion.

\section{$2.2 \quad$ Infravacuum Representations}

The physical idea of infrared clouds described at the beginning of this chapter is taken as a motivation of the following mathematical notion:

Definition: An irreducible representation $\left(\mathcal{H}_{I}, \pi_{I}\right)$ of $\mathfrak{A}_{0}$ is called an infravacuum representation if, for any $q \in \mathbf{V}_{+}$, the set $\tilde{\mathcal{S}}_{\pi_{0}}\left(D_{q}\right)$ contains some $\pi_{I^{-} \text {-normal state. }}$

In physical terms this means that, starting from the vacuum, one can create with an arbitrarily small amount $\epsilon=\sqrt{q^{2}}$ of energy some state in the infravacuum representation $\pi_{I}$. In the example of QED, such a state should be thought of as the soft photon part of an infrared cloud. The addition of the finitely many hard photons can be described by a quasilocal operation and thus does not change the infravacuum representation.

As an example for infravacuum representations, we mention the KPR representations, i.e., a class of non-Fock representations (of the free asymptotic electromagnetic field) devised by Kraus, Polley and Reents [5], cf. also [2:3], so as to be stable (up to unitary equivalence) under the bremsstrahlung produced in typical collision processes of charged particles. Infravacuum representations of this type will play a fundamental role in the example considered in Chapter 5 .

Remark: With the above definition, the vacuum $\pi_{0}$ itself is an infravacuum representation, which will be convenient in the sequel. However, the notion of infravacuum representations is tailored to theories with massless particles in the sense that in a purely massive theory (i.e., a theory where $\operatorname{sp} U_{0} \subset\{0\} \cup\left\{p \in \mathbf{V}_{+} \mid p^{2} \geq \mu^{2}\right\}$ for some $\mu>0$ ), $\pi_{0}$ would be the only infravacuum representation.

The following lemma collects some basic properties of any infravacuum representation. 
Lemma 2.3 Let $\pi_{I}$ be an infravacuum representation. Then:

$i$. For any $q \in \mathbf{V}_{+}, \tilde{\mathcal{S}}_{\pi_{0}}\left(D_{q}\right)$ contains a pure $\pi_{I}$-normal state.

ii. $\pi_{I}$ is a locally normal positive energy representation of $\mathfrak{A}_{0}$.

iii. For any bounded open set $\mathcal{O}$, the restriction of $\pi_{I}: \mathfrak{A}(\mathcal{O}) \longrightarrow \pi_{I}(\mathfrak{A}(\mathcal{O}))$ to uniformly bounded subsets of $\mathfrak{A}(\mathcal{O})$ is a homeomorphism with respect to the weak operator topologies.

Proof: Choose, for some $q \in \mathbf{V}_{+}$, a state $\omega \in \mathcal{S}_{\pi_{I}} \cap \tilde{\mathcal{S}}_{\pi_{0}}\left(D_{q}\right)$ and let $\left(\mathcal{H}_{\omega}, \pi_{\omega}, \Omega_{\omega}\right)$ be its GNS triple. Since $\omega \in \mathcal{S}_{\pi_{I}}, \pi_{\omega}$ is quasi-equivalent to the irreducible representation $\pi_{I}$. This implies that $\pi_{\omega}\left(\mathfrak{A}_{0}\right)^{\prime}$ is a type I factor and thus contains some minimal projection $P$. Obviously, $\left.\pi_{\omega}\right|_{P \mathcal{H}_{\omega}} \cong \pi_{I}$, which implies assertion ii by Lemma 2.1, i. Moreover, recalling $\Omega_{\omega} \in E_{\pi_{\omega}}\left(D_{q}\right) \mathcal{H}$, it also implies that the pure $\pi_{I^{-n o r m a l}}$ state $\omega_{P}:=\left(P \Omega_{\omega}, \pi_{\omega}(\cdot) P \Omega_{\omega}\right) /\left\|P \Omega_{\omega}\right\|^{2}$ fulfils

$$
\omega_{P} \in \mathcal{S}_{\pi_{I}}\left(D_{q}\right) \subset \mathcal{S}_{\pi_{\omega}}\left(D_{q}\right) \subset \tilde{\mathcal{S}}_{\pi_{\omega}}\left(D_{q}\right) \subset \tilde{\mathcal{S}}_{\pi_{0}}\left(2 D_{q}\right)
$$

Since $q$ was arbitrary, this proves Part i. Finally, Part iii follows by applying Corollary 7.1.16 of [24] to the maps $\pi_{I}: \mathfrak{A}(\mathcal{O}) \longrightarrow \pi_{I}(\mathfrak{A}(\mathcal{O}))$ which are isomorphisms of von Neumann algebras since $\pi_{I}$ is faithful and locally normal.

Let us remark that the property established in Part iii can even be strengthened to local unitary equivalence between $\pi_{0}$ and $\pi_{I}$ under the additional assumption that $\pi_{I}$ satisfies weak additivity.2] The reasoning for this goes along the following line: According to [II], there exist Reeh-Schlieder vectors in $\mathcal{H}_{0}$ and $\mathcal{H}_{I}$, i.e. the von Neumann algebras $\mathfrak{A}(\mathcal{O})$ and $\pi_{I}(\mathfrak{A}(\mathcal{O}))$ possess cyclic and separating vectors. From this, one obtains local unitary equivalence by applying Thm. 7.2.9 of [24]. One sees from these arguments that an attempt to establish unitary implementability of $\pi_{I}$ on an algebra pertaining to an unbounded region ( $\operatorname{such}$ as $\mathcal{O}^{\prime}$ ) would fail because $\pi_{I}$ need not be normal on such a region. In the applications to superselection theory we have in mind, this situation will indeed be the interesting case.

To conclude this section about general properties of infravacuum representations, we want to compare the nets $\tilde{\mathcal{S}}_{\pi_{0}}(\cdot)$ and $\tilde{\mathcal{S}}_{\pi_{I}}(\cdot)$ of states. In the next proposition, we will have to make the additional assumption that the vacuum state is unique in the sense that, for some $q \in \mathbf{V}_{+}, \omega_{0}$ is the only vacuum state in $\tilde{\mathcal{S}}_{\pi_{0}}\left(D_{q}\right)$.

Proposition 2.4 Let $\pi_{I}$ be an infravacuum representation. Then we have, for any $D$ and any $q \in \mathbf{V}_{+}$:

$$
\tilde{\mathcal{S}}_{\pi_{I}}(D) \subset \tilde{\mathcal{S}}_{\pi_{0}}\left(D+D_{q}-D_{q}\right)
$$

If, moreover, the defining representation $\pi_{0}$ satisfies $C_{\sharp}$ and if the vacuum state is unique in the sense explained above, then also the converse is true, namely

$$
\tilde{\mathcal{S}}_{\pi_{0}}(D) \subset \tilde{\mathcal{S}}_{\pi_{I}}\left(D+D_{q}-D_{q}\right)
$$

Proof: Using Lemma 2.3, the first statement follows immediately from Lemma 2.1, iii. To prove the second statement, we will first use the two additional assumptions to show $\omega_{0} \in$ $\tilde{\mathcal{S}}_{\pi_{I}}\left(D_{q}\right)$ for any $q \in \mathbf{V}_{+}$. This is done as follows. Take some $\omega \in \mathcal{S}_{\pi_{I}} \cap \tilde{\mathcal{S}}_{\pi_{0}}\left(D_{q}\right)$. It is easy

\footnotetext{
${ }^{2}$ If additivity and not merely weak additivity were assumed for $\pi_{0}$, additivity and hence weak additivity would follow for $\pi_{I}$ by local normality.
} 
to see that this implies $\omega \in \tilde{\mathcal{S}}_{\pi_{I}}\left(D_{q}\right)$. Now consider the family $\left(\omega_{L}\right)_{L>0}$ of states defined by $\omega_{L}:=\frac{1}{\left|V_{L}\right|} \int_{V_{L}} d^{1+s} x \omega \circ \alpha_{x}$, where $V_{L}:=[-L, L]^{(1+s)} \subset \mathbb{R}^{1+s}$. Since $\omega_{L} \in \tilde{\mathcal{S}}_{\pi_{I}}\left(D_{q}\right)$, any weak limit $\tilde{\omega}$ of this family is a weak limit of $\tilde{\mathcal{S}}_{\pi_{I}}\left(D_{q}\right)$. Now $\pi_{I}$ satisfies $\mathrm{C}_{\sharp}$ by Lemma 2.2, so the first part of that lemma can be applied to $\tilde{\omega}$ and yields $\tilde{\omega} \in \tilde{\mathcal{S}}_{\pi_{I}}\left(D_{q}\right)$. In particular, $\tilde{\omega}$ has positive energy. On the other hand, $\tilde{\omega}$ is translation invariant by construction, i.e. it is a vacuum state. Moreover, by the first part of the present proposition, $\tilde{\omega} \in \tilde{\mathcal{S}}_{\pi_{0}}\left(2 D_{q}\right)$. Now if $q$ is sufficiently small, the uniqueness assumption yields $\tilde{\omega}=\omega_{0}$ whence $\omega_{0} \in$ $\tilde{\mathcal{S}}_{\pi_{I}}\left(D_{q}\right)$. Trivially, this conclusion remains true for any $q$. With this information, the second statement follows again from Lemma 2.1, iii.

The above relation between the nets $\tilde{\mathcal{S}}_{\pi_{0}}(\cdot)$ and $\tilde{\mathcal{S}}_{\pi_{I}}(\cdot)$ can be expressed by saying that the transition energy (see [1:3] for this concept) between $\pi_{0}$ and $\pi_{I}$ vanishes.

Finally, let us note that Proposition 2.4 does not, in general, entail that one of the sets $\tilde{\mathcal{S}}_{\pi_{0}}(D)$ and $\tilde{\mathcal{S}}_{\pi_{I}}(D)$ is contained in the other because the nets $D \longmapsto \tilde{\mathcal{S}}_{\pi}(D)$ need not be regular from the outside (which would mean $\tilde{\mathcal{S}}_{\pi}(D)=\tilde{\mathcal{S}}_{\pi}(D):=\bigcap_{q} \tilde{\mathcal{S}}_{\pi}\left(D+D_{q}\right)$ ), but the outer regularised nets $\tilde{\mathcal{S}}_{\pi}$ coincide. (In contrast to this, the nets $\mathcal{S}_{\pi_{0}}$ and $\mathcal{S}_{\pi_{I}}$ are regular from the outside, as follows from the continuity of the spectral families $E_{0}$ and $E_{\pi_{I}}$.) 



\section{Chapter 3}

\section{Superselection Theory with General Localisation}

This chapter is devoted to a review of the mathematical description of superselection charges with general localisation properties. The objects of interest in superselection theory are the charged states of the given model. These states are subdivided into superselection sectors labeled by charge quantum numbers. Within one sector, the charge is fixed in the sense that its value cannot be modified by local operations.

A central aspect of the notion of charge in particle physics is that of localisation. It presupposes that there exist some states which are identified as uncharged and therefore serve as a background. Charged states are then defined in comparison to that background, and it is consistent to say that in such a state the charge is localised in some spacetime region $X$ if its effects cannot be distinguished from the background by measurements performed in the causal complement $X^{\prime}$ of $X$.

Within one sector the localisation region of the charge need of course not be fixed: for each member $X$ of some class of possible localisation regions, the sector contains states in which the charge is localised in $X$. This is expressed by saying that the charge is transportable.

Starting from the aspects of localisation and transportability of charges, superselection theory now describes several basic features of particle physics. In particular it provides a formulation of the addition (composition) of charges, it explains the particle-antiparticle symmetry (charge conjugation) and accounts for the Bose-Fermi alternative (classification of statistics). That the localisation properties are central to these issues may not be surprising from an operational point of view, and heuristic ideas based on the picture of charges as point particles enter into the theory at several places.

In their pioneering work [7, 8], Doplicher, Haag and Roberts considered pointlike charges. Compared to the vacuum, such charges can be localised in any compact region (at a given time). It has however been shown by Buchholz and Fredenhagen [ $\underline{9}]$ that even in purely massive theories, charges may in general only be localisable in spacelike cones. Such a localisation also is supposed to cover the physically interesting case of electric or magnetic charges, but only with the proviso that the vacuum is replaced with some other background containing a suitably fluctuating infrared cloud. As suggested by Buchholz in [6], the set of superselection sectors in front of such a background ought to be labeled by the value of the total electric charge (which corresponds to the total flux 
of the asymptotic Coulomb field by Gauss' law), but not - as it is the case in front of the vacuum - by the detailed shape of the asymptotic electric field. Such a phenomenon indeed occurs in the model of the massless free field, as will be seen in Chapter 5. This model possesses charges localisable in "upright" spacelike cones (in a fixed Lorentz system) and even contains charges which are only localisable in "upright opposite spacelike cones" (see Section 3.2 for a description of these classes of regions).

In view of the diversity of possible localisation properties of charges we believe it worthwhile to go through the analysis step by step paying special attention to the topological properties of localisation regions. The exposition will be quite similar to parts of [9], but we will put more emphasis on the functorial aspects of the construction. A nice account of superselection theory for pointlike charges can also be found in [25].

For the sake of clarity and self-containedness, the relevant categorical notions have been collected in Appendix A; it might be advisable to read this appendix parallel to the present chapter.

\subsection{Assumptions and Basic Properties}

As indicated above, the starting point for superselection theory is a background and a set $\mathcal{X}$ consisting of localisation regions. Each $X \in \mathcal{X}$ is assumed to be a nonempty causally complete open subset $X \subset \mathbb{R}^{1+s}$ of Minkowski spacetime. The set $\mathcal{X}$ is assumed to be sufficiently rich in the sense that that for each $X \in \mathcal{X}$ there is some $\tilde{X}$ contained in $X^{\prime}$ and that each bounded region $\mathcal{O}$ is contained in some $Y \in \mathcal{X}$. Moreover the connectedness assumptions $\mathbf{c 1}, \mathbf{c 2}, \mathbf{c 3}$ and the enlargement assumption e will be made for the set $\mathcal{X}$, but as these properties will not be needed in the very first steps, their explicit statement is postponed to Section 3.2.

The background is described by an irreducible representation $\pi_{I}: \mathfrak{A}_{0} \longrightarrow \mathcal{B}\left(\mathcal{H}_{I}\right)$ of the quasilocal algebra. It generates a net²

$$
X \longmapsto \mathfrak{A}_{I}(X):=\pi_{I}(\mathfrak{A}(X)), \quad X \in \mathcal{X}
$$

of concrete $\mathrm{C}^{*}$-algebras on $\mathcal{H}_{I}$ satisfying isotony and locality. We will write $\mathfrak{A}_{I}:=\pi_{I}\left(\mathfrak{A}_{0}\right)$ and $\mathfrak{A}_{I}\left(X^{\prime}\right):=\pi_{I}\left(\mathfrak{A}\left(X^{\prime}\right)\right)$. Notice that $\mathfrak{A}_{I}=\bar{\bigcup}_{X} \mathfrak{A}_{I}(X){ }^{\|\cdot\|}$.

The following algebraic assumptions assure that $\pi_{I}$ can be regarded as a background in a very general sense:

b1 The net $\mathfrak{A}_{I}$ has property B, i.e., for each $Y \in \mathcal{X}$ there is some $X \subset Y$ such that each nonzero projection in $\mathfrak{A}_{I}\left(X^{\prime}\right)^{\prime}$ is the final projection of some isometry in $\mathfrak{A}_{I}\left(Y^{\prime}\right)^{\prime}$.

b2 The net $\mathfrak{A}_{I}$ fulfils duality, i.e., $\mathfrak{A}_{I}\left(X^{\prime}\right)^{\prime}=\mathfrak{A}_{I}(X)^{\prime \prime}$ for all $X \in \mathcal{X}$.

The notation $\pi_{I}$ is chosen for the background representation since this might be an infravacuum representation in the sense of Chapter 2 (or the defining representation $\pi_{0}$ as a special case), but such a specific assumption will only be made in Chapter 1 . Property $\mathrm{B}$ is quite a mild assumption: it is fulfilled if $\pi_{I}$ has positive energy and if weak additivity holds for $\pi_{I}$, cf. [1T].

\footnotetext{
${ }^{1}$ In the sequel, symbols like $X, X_{0}, Y, \tilde{Y}, \ldots$ will always denote elements of $\mathcal{X}$ and $X^{\prime}, X_{0}^{\prime}, Y^{\prime}, \tilde{Y}^{\prime}, \ldots$ their spacelike complements.

${ }^{2}$ By abuse of language, we use the term "net" for families indexed by $\mathcal{X}$ although this need not be a directed set.
} 
The duality assumption, on the other hand, is more severe. Here it is made for simplicity, and it could be weakened to essential duality (i.e., the requirement that the net $X \longmapsto \mathfrak{A}_{I}\left(X^{\prime}\right)^{\prime}$ fulfil locality) for the purposes of superselection theory [26]. As long as the regions $X$ are intersections of wedge regions, essential duality can be deduced from duality for wedge regions, which in turn is a reasonable assumption, [27]. In other cases however (including that of opposite spacelike cones), there seem to be no general arguments implying essential duality automatically.

Starting from these data, one now studies the set of representations $\left(\mathcal{H}_{\pi}, \pi\right)$ of $\mathfrak{A}_{0}$ which are localisable in each $X \in \mathcal{X}$ in front of the background $\pi_{I}$, i.e., the set

$$
\operatorname{DHR}\left(\mathcal{X}, \pi_{I}\right):=\left\{\pi:\left.\mathfrak{A}_{0} \longrightarrow \mathcal{B}\left(\mathcal{H}_{\pi}\right)|\pi|_{\mathfrak{A}\left(X^{\prime}\right)} \cong \pi_{I}\right|_{\mathfrak{A}\left(X^{\prime}\right)} \text { for each } X \in \mathcal{X}\right\}
$$

As explained in [ [7], assumption b1 implies that $\operatorname{DHR}\left(\mathcal{X}, \pi_{I}\right)$ is closed under subrepresentations and finite direct sums. But due to the net structure of $X \longmapsto \mathfrak{A}_{I}(X)$ and the duality assumption b2, this set has a much richer structure than what appears at first sight. The great achievement of $[\boldsymbol{Z}, \boldsymbol{8}]$ is to make this structure manifest. The key idea which allows the local information encoded in the net $\mathfrak{A}_{I}(\cdot)$ to be used is to relate $\operatorname{DHR}\left(\mathcal{X}, \pi_{I}\right)$ to the set of transportable localised homomorphisms $\rho: \mathfrak{A}_{I} \longrightarrow \mathcal{B}\left(\mathcal{H}_{I}\right)$, a notion defined as follows:

Definition: Let $\operatorname{Hom}\left(\mathfrak{A}_{I}, \mathcal{B}\left(\mathcal{H}_{I}\right)\right)$ denote the set of all unital $\mathrm{C}^{*}$-algebra homomorphisms $\rho: \mathfrak{A}_{I} \longrightarrow \mathcal{B}\left(\mathcal{H}_{I}\right)$. Two such homomorphisms $\rho_{1}$ and $\rho_{2}$ are said to be equivalent iff there is a unitary $v$ on $\mathcal{H}_{I}$ such that $\rho_{1}=\operatorname{Ad} v \circ \rho_{2}$. A homomorphism $\rho$ is called localised in $X \in \mathcal{X}$ iff it acts trivially on the subalgebra $\mathfrak{A}_{I}\left(X^{\prime}\right) \subset \mathfrak{A}_{I}$, and it is called transportable iff, for any $\tilde{X} \in \mathcal{X}$, there exists some equivalent homomorphism $\tilde{\rho}$ which is localised in $\tilde{X}$. We will denote with $\Delta(X)$ the set of all transportable homomorphisms localised in $X$ and let $\Delta=\bigcup_{X \in \mathcal{X}} \Delta(X)$.

As explained in Appendix A.1 (where the relevant notions and notations have been summarised), $\operatorname{Hom}\left(\mathfrak{A}_{I}, \mathcal{B}\left(\mathcal{H}_{I}\right)\right)$ can be viewed in a natural way as the set of objects of a $\mathrm{W}^{*}$-category; the subsets $\Delta$ and $\Delta(X)$ then define full $\mathrm{W}^{*}$-subcategories. Without any risk of confusion, we shall denote these $\mathrm{W}^{*}$-categories with the same symbols $\operatorname{Hom}\left(\mathfrak{A}_{I}, \mathcal{B}\left(\mathcal{H}_{I}\right)\right)$, $\Delta$ and $\Delta(X)$, respectively. The sets of morphisms from an object $\sigma$ to an object $\tau$ will be denoted with $I(\sigma, \tau)$; we will use $t: \sigma \rightarrow \tau$ synonymously to $t \in I(\sigma, \tau)$. Such a morphism is a triple $t=(\tau, t, \sigma)$, where the intertwining operator $t \in \mathcal{B}\left(\mathcal{H}_{I}\right)$ has to satisfy $\tau(a) t=t \sigma(a)$ for all $a \in \mathfrak{A}_{I}$. Since we feel the need for a notational distinction between a morphism and the corresponding intertwiner, we consider $t \longmapsto t$ as an isometric linear map from $I(\sigma, \tau)$ into $\mathcal{B}\left(\mathcal{H}_{I}\right)$. If needed, the image of this map will be denoted with $I(\sigma, \tau)$.

In this new perspective the map $X \longmapsto \Delta(X)$, formerly an isotonous net of subsets of (the set) $\Delta$ now becomes an isotonous net of full subcategories of (the $\mathrm{W}^{*}$-category) $\Delta$. It follows (see Lemma 3.1 below) from the corresponding property of $\operatorname{DHR}\left(\mathcal{X}, \pi_{I}\right)$ that $\Delta$ is closed under subobjects and finite direct sums. Moreover, as the objects $\rho \in \Delta$ are transportable homomorphisms, all these subcategories $\Delta(X)$ are equivalent to $\Delta$. The object $\iota: \mathfrak{A}_{I} \hookrightarrow \mathcal{B}\left(\mathcal{H}_{I}\right)$ is the only object contained in each of these subcategories and is irreducible since, by the irreducibility of $\pi_{I}, I(\iota, \iota)=\mathcal{B}\left(\mathcal{H}_{I}\right) \cap \mathfrak{A}_{I}^{\prime}=\mathbb{C} \mathbf{1}$.

It is necessary at this point to verify that no information is lost in the process of going over from $\operatorname{DHR}\left(\mathcal{X}, \pi_{I}\right)$ to $\Delta$. This is the role of the following lemma: 


\section{Lemma 3.1}

i. Let $\pi \in \operatorname{DHR}\left(\mathcal{X}, \pi_{I}\right)$ be given and let $X \in \mathcal{X}$. Then there is some $\rho \in \Delta(X)$ such that $\pi \cong \rho \circ \pi_{I}$.

ii. Let $\rho \in \Delta$. Then $\rho \circ \pi_{I} \in \operatorname{DHR}\left(\mathcal{X}, \pi_{I}\right)$.

Proof: i. By assumption, there exists some unitary $V_{X}: \mathcal{H}_{\pi} \longrightarrow \mathcal{H}_{I}$ such that one has $\pi_{I}=\operatorname{Ad} V_{X} \circ \pi$ on $\mathfrak{A}\left(X^{\prime}\right)$. Since $\pi_{I}$ is faithful, $\rho:=\operatorname{Ad} V_{X} \circ \pi \circ \pi_{I}^{-1}$ is a well-defined element of $\operatorname{Hom}\left(\mathfrak{A}_{I}, \mathcal{B}\left(\mathcal{H}_{I}\right)\right)$ localised in $X$ and satisfying $\pi \cong \rho \circ \pi_{I}$. By repeating this argument for any other $\tilde{X} \in \mathcal{X}$, one obtains an equivalent homomorphism localised in $\tilde{X}$. Hence $\rho$ is transportable.

ii. Let $\rho \in \Delta$ and $X \in \mathcal{X}$. Then there is some $\tilde{\rho} \in \Delta(X)$ satisfying $\tilde{\rho} \cong \rho$. On $\mathfrak{A}\left(X^{\prime}\right)$, it follows in particular that $\rho \circ \pi_{I} \cong \tilde{\rho} \circ \pi_{I} \cong \pi_{I}$. Since $X$ was arbitrary, this proves the assertion.

Remark: In the obvious way, one can also consider $\operatorname{DHR}\left(\mathcal{X}, \pi_{I}\right)$ as the set of objects of a $\mathrm{W}^{*}$-category. Lemma 3.1 then amounts to saying that $\rho \longmapsto \rho \circ \pi_{I}$ is a $\mathrm{W}^{*}$-functor from $\Delta$ to $\operatorname{DHR}\left(\mathcal{X}, \pi_{I}\right)$ whose image is a full subcategory equivalent to (all of) $\operatorname{DHR}\left(\mathcal{X}, \pi_{I}\right)$. Thus if one is interested in isomorphism classes of objects only, $\Delta$ and $\operatorname{DHR}\left(\mathcal{X}, \pi_{I}\right)$ become indistinguishable. The crucial fact however is that $\Delta$ can be equipped with a much richer structure than $\operatorname{DHR}\left(\mathcal{X}, \pi_{I}\right)$. In the author's opinion, the analysis of $\Delta$ (instead of $\left.\operatorname{DHR}\left(\mathcal{X}, \pi_{I}\right)\right)$ is more than a mere trick with the scope of eventually equipping the quotient $\operatorname{DHR}\left(\mathcal{X}, \pi_{I}\right) / \cong$ with the corresponding additional structure, it seems rather that it is an instance of so-called categorification (cf. the remarks in [28] on that topic) which embodies the physical fact that differently localised charges are equivalent (in the sense of charge conservation) but not identical.

The duality requirement b2, finally, is of great technical importance since, via the following lemma, it will allow the monoidal products to be introduced.

\section{Lemma 3.2}

i. Let $\rho \in \Delta(X), X \in \mathcal{X}$. Then $\rho\left(\mathfrak{A}_{I}(X)\right) \subset \mathfrak{A}_{I}(X)^{\prime \prime}$.

ii. Let $\sigma, \tau \in \Delta(X), X \in \mathcal{X}$. Then $I(\sigma, \tau) \subset \mathfrak{A}_{I}(X)^{\prime \prime}$.

Proof: From the definition of the localisation properties and the locality of the net $X \longmapsto$ $\mathfrak{A}_{I}(X)$, it follows in a straightforward way that inclusions analogous to the asserted ones hold if the right-hand sides are replaced with $\mathfrak{A}_{I}\left(X^{\prime}\right)^{\prime}$. By postulating that this algebra not be larger than the weak closure of $\mathfrak{A}_{I}(X)$, the duality requirement implies the statement.

\subsection{Connectedness and Enlargement Properties of $\mathcal{X}$}

This section will deal with the properties of the set $\mathcal{X}$ which will be used in the subsequent analysis but which have not yet been stated explicitly. The connectedness properties rely on the notions of paths and pathwise connectedness which we briefly recall. First, note that the natural half-ordering of $\mathcal{X}$ by the inclusion of subsets of $\mathbb{R}^{1+s}$ induces a half-ordering on any subset of $\mathcal{X} \times \mathcal{X}$, namely $\left(X_{1}, X_{2}\right) \subset\left(Y_{1}, Y_{2}\right)$ iff $X_{1} \subset Y_{1}$ and $X_{2} \subset Y_{2}$. Second, recall that a path of length $N$ in a half-ordered set $(A, \subset$ ) (for the present purposes, $A$ will be either a subset of $\mathcal{X}$ or of $\mathcal{X} \times \mathcal{X})$ is a sequence $a_{0}, a_{1}, \ldots, a_{N}$ of elements of $A$ such 
that one has, for each $j=1, \ldots, N$, either $a_{j-1} \subset a_{j}$ or $a_{j} \subset a_{j-1}$. Such a path is said to go from $a_{0}$ to $a_{N}$, and the set $A$ is called connected iff any two of its elements can be joined by a path of finite length. Using, for any $R \subset \mathbb{R}^{1+s}$, the notations

$$
\begin{aligned}
\mathcal{X}(R) & :=\{X \in \mathcal{X} \mid X \subset R\}, \\
\mathcal{X}_{\times}^{(2)}(R) & :=\left\{\left(X_{1}, X_{2}\right) \in \mathcal{X}(R) \times \mathcal{X}(R) \mid X_{1} \times X_{2}\right\},
\end{aligned}
$$

the connectedness requirements imposed on the set $\mathcal{X}$ of localisation regions can be formulated as:

c1 The set $\mathcal{X}$ is connected.

c2 For any $X \in \mathcal{X}$, the set $\mathcal{X}_{\times}^{(2)}(X)$ is nonempty and connected.

c3 For any $X \in \mathcal{X}$, there exist sequences $X_{n}$ and $Y_{n}$ satisfying $\left(X, X_{n}\right) \in \mathcal{X}_{\times}^{(2)}\left(Y_{n}\right)$ $(n \in \mathbb{N})$ and such that $X_{n}$ tends to spacelike infinity in the following sense: For each bounded region $\mathcal{O}$, there is some $n_{\mathcal{O}} \in \mathbb{N}$ such that $X_{n_{\mathcal{O}}} \times \mathcal{O}$.

In view of its verification in concrete situations, assumption $\mathbf{c 2}$ can be reduced to two more elementary topological properties:

Proposition 3.3 For assumption c2 to be valid, it is sufficient that the following two properties be fulfilled:

c2 $_{1}$ For any $X \in \mathcal{X}$ and $X_{0} \subset X$, there exists some $X_{00} \subset X_{0}$ such that $\mathcal{X}\left(X \cap X_{00}^{\prime}\right)$ is nonempty and connected.

c2 $_{2}$ For any $X \in \mathcal{X}$ and $X_{1}, X_{2} \subset X$, there exist some $X_{10} \subset X_{1}$ and $X_{20} \subset X_{2}$ such that $\mathcal{X}\left(X \cap X_{10}^{\prime} \cap X_{20}^{\prime}\right)$ is nonempty.

Proof: It follows from $\mathbf{c 2}_{1}$ (with, say, $X_{0}=X$ ) that $\mathcal{X}_{\times}^{(2)}(X)$ is nonempty. Now let $\left(Y_{1}, \tilde{Y}_{1}\right),\left(Y_{2}, \tilde{Y}_{2}\right) \in \mathcal{X}_{\mathbf{x}}^{(2)}(X)$. By $\mathbf{c 2} 2_{2}$, there exist $Y_{10} \subset Y_{1}$ and $Y_{20} \subset Y_{2}$ such that the set $\mathcal{X}\left(X \cap Y_{10}^{\prime} \cap Y_{20}^{\prime}\right)=\mathcal{X}\left(X \cap Y_{10}^{\prime}\right) \cap \mathcal{X}\left(X \cap Y_{20}^{\prime}\right)$ is nonempty. By $\mathbf{c 2} 2_{1}, Y_{10}$ and $Y_{20}$ can be chosen sufficiently small such that, in addition, $\mathcal{X}\left(X \cap Y_{10}^{\prime}\right)$ and $\mathcal{X}\left(X \cap Y_{20}^{\prime}\right)$ are connected. This means that there exists some $\tilde{Y}_{12} \in \mathcal{X}\left(X \cap Y_{10}^{\prime}\right) \cap \mathcal{X}\left(X \cap Y_{20}^{\prime}\right)$ which can be joined from $\tilde{Y}_{j} \in \mathcal{X}\left(X \cap Y_{j}^{\prime}\right) \subset \mathcal{X}\left(X \cap Y_{j 0}^{\prime}\right)$ by a finite path in $\mathcal{X}\left(X \cap Y_{j 0}^{\prime}\right), j=1,2$, and $\tilde{Y}_{12}$ can be chosen so small that $\mathcal{X}\left(X \cap \tilde{Y}_{12}^{\prime}\right)$ is connected. But this implies there exist finite paths in $\mathcal{X}_{\times}^{(2)}(X)$ from $\left(Y_{10}, \tilde{Y}_{1}\right)$ to $\left(Y_{10}, \tilde{Y}_{12}\right)$, from $\left(Y_{10}, \tilde{Y}_{12}\right)$ to $\left(Y_{20}, \tilde{Y}_{12}\right)$ and from $\left(Y_{20}, \tilde{Y}_{12}\right)$ to $\left(Y_{20}, \tilde{Y}_{2}\right)$. Since $\left(Y_{j 0}, \tilde{Y}_{j}\right) \subset\left(Y_{j}, \tilde{Y}_{j}\right)$ this proves the assertion.

The last assumption regarding the set $\mathcal{X}$ is the enlargement property:

e The set $\mathcal{X}$ admits an enlargement $\left(e_{n}\right)_{n \in \mathbb{N}}$, that is, a sequence of isotonous maps $e_{n}: \mathcal{X} \longrightarrow \mathcal{X}, n \in \mathbb{N}$, such that one has, for each $X \in \mathcal{X}$,

$$
X \subset e_{1}(X) \subset e_{2}(X) \subset e_{3}(X) \subset \ldots \quad \text { and } \quad \bigcup_{n} e_{n}(X)=\mathbb{R}^{1+s} .
$$


Notice that by a compactness argument, one can show that (given $X$ ), each bounded set $\mathcal{O} \subset \mathbb{R}^{1+s}$ is contained in $e_{n}(X)$ for sufficiently large $n$.

The enlargement property $\mathbf{e}$ and the connectedness property $\mathbf{c 1}$ will enter the discussion of Section 3.3 where the monoidal product is defined. As it turns out, the above conditions imposed on $\left(e_{n}\right)_{n \in \mathbb{N}}$ could be weakened substantially, but we maintain them as stated, since they can be fulfilled in a wide class of examples (see below). Property c2 will be used in Section 3.4 for the construction of a symmetry; c3, finally, is needed for establishing the existence of conjugates in Section 3.5. Also notice that the formulation of these properties permits an immediate generalisation from Minkowski space to other (globally hyperbolic) spacetimes.

Some examples for sets $\mathcal{X}$ of localisation regions and for an enlargement are in order at this point. Besides the two classical ones, let us mention a less standard one which appears in the example discussed in Chapter 5 (see p. 66):

A The set of all double cones $c+\mathcal{O}_{a} \equiv \mathcal{O}_{c+a, c-a}=\left(c+a-\mathbf{V}_{+}\right) \cap\left(c-a+\mathbf{V}_{+}\right)$(where $\left.c \in \mathbb{R}^{1+s}, a \in \mathbf{V}_{+}\right)$if the spacetime dimension fulfils $1+s \geq 1+2$.

B The set of all (causally complete) spacelike cones $c+\mathcal{S}_{a_{ \pm}}=c+\mathbb{R}_{>0} \mathcal{O}_{a_{+}, a_{-}}$(where $c \in \mathbb{R}^{1+s}, a_{+}, a_{-} \in \mathbb{R}^{1+s}$ such that $a_{+}^{2}=a_{-}^{2}=-1$ and $\left.a_{+}-a_{-} \in \mathbf{V}_{+}\right)$if $1+s \geq 1+3$. (Notice that the condition $a_{+}^{2}=a_{-}^{2}$ guarantees that $\mathcal{S}_{a_{ \pm}}$is causally complete and that $a_{ \pm}^{2}=-1$ makes $a_{ \pm}$unique.)

C The set of all opposite spacelike cones $c+\mathcal{D}_{b, a_{ \pm}}:=\left(\left(c+b+\mathcal{S}_{a_{ \pm}}\right) \cup\left(c-b-\mathcal{S}_{a_{ \pm}}\right)\right)^{\prime \prime}$ (where $b, c \in \mathbb{R}^{1+s}$ and $a_{ \pm}$are as in B) again if $1+s \geq 1+3$.

(The shape of $\mathcal{D}_{b, a_{ \pm}}$is particularly simple in the special case when $b \in \mathbb{R}\left(a_{+}+a_{-}\right)$. Notice also that $-b \times \mathcal{S}_{a_{ \pm}}$is admitted. In this case, the set $\left(b+\mathcal{S}_{a_{ \pm}}\right) \cup\left(-b-\mathcal{S}_{a_{ \pm}}\right)$ has two connected components and, being causally complete, equals $\mathcal{D}_{b, a_{ \pm}}$.)

If a Lorentz system is fixed (e.g. by a given unit vector $e \in \mathbf{V}_{+}$), then one obtains other sets $\mathcal{X}$ of localisation regions by restriction to the upright elements. Localisation in such regions plays a role in models without Lorentz covariance.

A' The set of all upright double cones: like A, but with $a \in \mathbb{R}_{>0} e$.

B' The set of all upright spacelike cones: like $\mathrm{B}$, but with $a_{+}-a_{-} \in \mathbb{R}_{>0} e$.

C' The set of all upright opposite spacelike cones: like C, but with $a_{+}-a_{-} \in \mathbb{R}_{>0} e$.

As to the proofs that the properties $\mathbf{c 1}, \mathbf{c 2}, \mathbf{c 3}$ are satisfied in each of these six examples, the following remarks should be sufficient.

- In the cases A and A', c1 and c3 are verified directly, and c2 is established via Prop. 3.3. The properties $\mathbf{c 2} 2_{1}$ and $\mathbf{c} \mathbf{2}_{2}$ which appear there can in turn be reduced to topological properties of points (representing infinitesimally small double cones) in Minkowski spacetime. Finally, an enlargement in the sense of $\mathbf{e}$ is simply given by $e_{n}\left(c+\mathcal{O}_{a}\right)=c+n \mathcal{O}_{a}, n \in \mathbb{N}$.

- In the cases B and B' it is very convenient (cf. the Appendix of [2.: ]) to reduce the proof of $\mathbf{c 1}, \mathbf{c 2} 2_{1}, \mathbf{c 2} 2_{2}, \mathbf{c 3}$ to corresponding properties $\left.\}\right]$ of the set $\mathcal{X}_{0}:=\left\{\left(c+\mathcal{S}_{a_{ \pm}}\right) \in\right.$

\footnotetext{
${ }^{3}$ In the case of $\mathbf{c 3}$, the corresponding property reads $\mathbf{c 3}_{0}$ : For any $X \in \mathcal{X}_{0}$, there exist $\tilde{X}, Y \in \mathcal{X}_{0}$ such that $(X, \tilde{X}) \in \mathcal{X}_{0, \times}^{(2)}(Y)$.
} 
$\mathcal{X} \mid c=0\}$ of spacelike cones attached to the origin of Minkowski space. Since $\mathcal{X}_{0}$ can be identified in a natural way with the set of double cones on the hyperboloid $\left\{x \in \mathbb{R}^{1+s} \mid x^{2}=-1\right\}$ (or - in the upright case - more naturally with the set of open pointed convex cones in the euclidean space $\mathbb{R}^{s}$ ), the verification of $\mathbf{c 1}, \mathbf{c 2} \mathbf{2}_{1}$, $\mathbf{c 2} 2_{2}, \mathbf{c} 3_{0}$ for $\mathcal{X}_{0}$ is very similar to the cases $\mathrm{A}$ and $\mathrm{A}^{\prime}$ above. Using the connections between the relations $\subset$ and $\boldsymbol{X}$ in $\mathcal{X}_{0}$ and in $\mathcal{X}$, viz. (with $\mathcal{S}_{1}, \mathcal{S}_{2} \in \mathcal{X}_{0}$ )

$$
\begin{aligned}
& c_{1}+\mathcal{S}_{1} \subset c_{2}+\mathcal{S}_{2} \quad \Longleftrightarrow \quad c_{1}-c_{2} \in \overline{\mathcal{S}_{2}} \quad \text { and } \quad \mathcal{S}_{1} \subset \mathcal{S}_{2}, \\
& c_{1}+\mathcal{S}_{1} \times c_{2}+\mathcal{S}_{2} \quad \Longleftrightarrow \quad c_{1}-c_{2} \in-\mathcal{S}_{1}^{\prime} \cap \mathcal{S}_{2}^{\prime} \quad \text { and } \quad \mathcal{S}_{1} \times \mathcal{S}_{2},
\end{aligned}
$$

it is then possible to deduce $\mathbf{c 1}, \mathbf{c 2} \mathbf{2}_{1}, \mathbf{c 2} \mathbf{2}_{2}, \mathbf{c} \mathbf{3}$ for $\mathcal{X}$ from their respective counterparts for $\mathcal{X}_{0}$. (For instance $\mathbf{c 3}$ follows from $\mathbf{c 3}_{0}$ like this: assume $X=c+\mathcal{S}$ to be given, $\mathcal{S} \in \mathcal{X}_{0}$. By $\mathbf{c 3}_{0}$, one can choose $\mathcal{S}_{0}, \tilde{\mathcal{S}} \in \mathcal{X}_{0}$ such that $\left(\mathcal{S}, \mathcal{S}_{0}\right) \in \mathcal{X}_{0, \times}^{(2)}(\tilde{\mathcal{S}})$. For any $x \in \mathcal{S}_{0}$, the sequences $X_{n}:=c+n x+\mathcal{S}_{0}$ and $Y_{n}:=c+\tilde{\mathcal{S}}, n \in \mathbb{N}$ then have the properties claimed in $\mathbf{c 3}$.)

Moreover, an enlargement $\left(e_{n}\right)_{n \in \mathbb{N}}$ can be constructed explicitly: the assignment

$$
e_{n}\left(c+\mathcal{S}_{a_{ \pm}}\right):=c-n x_{a_{ \pm}}+\mathcal{S}_{a_{ \pm}}
$$

satisfies $X \subset e_{1}(X) \subset e_{2}(X) \subset \ldots$ and $\bigcup_{n} e_{n}(X)=\mathbb{R}^{1+s}$ for each $X \in \mathcal{X}$ whenever $x_{a_{ \pm}} \in \mathcal{S}_{a_{ \pm}}$, and choosing $x_{a_{ \pm}}:=\int_{\mathcal{O}_{a_{+}, a_{-}}} \mathrm{d}^{s+1} y y$ (which is an element of $\mathbb{R}_{>0} \mathcal{O}_{a_{+}, a_{-}}$ since $\mathcal{O}_{a_{+}, a_{-}}$is convex), one also obtains isotony. To see this, take $\mathcal{S}_{1}=\mathbb{R}_{>0} \mathcal{O}_{a_{+}, a_{-}}$ and $\mathcal{S}_{2}=\mathbb{R}_{>0} \mathcal{O}_{b_{+}, b_{-}}$and assume $c_{1}+\mathcal{S}_{1} \subset c_{2}+\mathcal{S}_{2}$, i.e., $c_{1}-c_{2} \in \overline{\mathcal{S}_{2}}$ and $\mathcal{O}_{a_{+}, a_{-}} \subset$ $\mathcal{O}_{b_{+}, b_{-}}$. It is then easily seen that $e_{n}\left(c_{1}+\mathcal{S}_{1}\right) \subset e_{n}\left(c_{2}+\mathcal{S}_{2}\right)$ for all $n \in \mathbb{N}$ is equivalent to $x_{b_{ \pm}}-x_{a_{ \pm}} \in \overline{\mathcal{S}_{2}}$. But this is indeed the case, since because of $\mathcal{O}_{a_{+}, a_{-}} \subset \mathcal{O}_{b_{+}, b_{-}}$ one has $x_{b_{ \pm}}-x_{a_{ \pm}}=\int_{\mathcal{O}_{b_{+}, b_{-}} \backslash \mathcal{O}_{a_{+}, a_{-}}} \mathrm{d}^{s+1} y y \in \mathbb{R}_{\geq 0} \operatorname{conv}\left(\mathcal{O}_{b_{+}, b_{-}} \backslash \mathcal{O}_{a_{+}, a_{-}}\right) \subset \overline{\mathcal{S}_{2}}$.

- In the cases C and C', the properties c1, c2, c3 can either be deduced from the cases B and B', or they can be established independently (but of course with similar methods as for B and B'). Regarding $\mathbf{c 1}$ and $\mathbf{c 2}$, the former way seems to be more direct. Denoting with $\mathcal{X}_{\mathrm{S}}$ the subset of all opposite spacelike cones $c+\mathcal{D}_{b, a_{ \pm}}$with two components (the index S stands for "separated"), it follows from the cases B and B' that the properties $\mathbf{c 1}$ and $\mathbf{c 2}$ are fulfilled for $\mathcal{X}_{\mathrm{S}}$. But since each $c+\mathcal{D}_{b, a_{ \pm}} \in$ $\mathcal{X}$ contains some $\mathcal{D}_{\tilde{b}, a_{ \pm}} \in \mathcal{X}_{\mathrm{S}}$ as a subset, it is readily seen that $\mathbf{c 1}$ and $\mathbf{c 2}$ are also fulfilled for $\mathcal{X}$. Property $\mathbf{c 3}$, on the other hand, can more easily be derived independently, drawing again on the property $\mathbf{c} \mathbf{3}_{0}$ for the set of ordinary spacelike cones attached to the origin.

Finally, the enlargement $\left(e_{n}\right)_{n \in \mathbb{N}}$ from cases B and B' can be used to define an enlargement $\left(\tilde{e}_{n}\right)_{n \in \mathbb{N}}$ in the present cases by setting

$$
\tilde{e}_{n}\left(c+\mathcal{D}_{b, a_{ \pm}}\right):=\left(e_{n}\left(c+b+\mathcal{S}_{a_{ \pm}}\right) \cup e_{n}\left(c-b-\mathcal{S}_{a_{ \pm}}\right)\right)^{\prime \prime} .
$$

It follows immediately from the corresponding properties of $e_{n}$ that $n \longmapsto \tilde{e}_{n}(X)$ is a sequence in $\mathcal{X}$ which increases monotonously to $\mathbb{R}^{1+s}$ for each $X=c+\mathcal{D}_{b, a_{ \pm}} \in$ $\mathcal{X}$. The issue of isotony of each map $\tilde{e}_{n}: \mathcal{X} \longrightarrow \mathcal{X}$ is a bit more subtle, since $\left(c_{1}+b_{1}+\mathcal{S}_{1}\right) \vee\left(c_{1}-b_{1}-\mathcal{S}_{1}\right) \subset\left(c_{2}+b_{2}+\mathcal{S}_{2}\right) \vee\left(c_{2}-b_{2}-\mathcal{S}_{2}\right)$ (with the notation $\left.A \vee B:=(A \cup B)^{\prime \prime}\right)$ does not entail termwise inclusion, in general. One has of course 
$\left(c_{1} \pm\left(b_{1}+\mathcal{S}_{1}\right)\right) \subset\left(c_{2}+b_{2}+\mathcal{S}_{2}\right) \vee\left(c_{2}-b_{2}-\mathcal{S}_{2}\right)$ and (after a possible substitution $\left.\left(b_{1}, \mathcal{S}_{1}\right) \mapsto\left(-b_{1},-\mathcal{S}_{1}\right)\right)$ also $\mathcal{S}_{1} \subset \mathcal{S}_{2}$. It is then the explicit form of $e_{n}$ (with $x_{j} \in \mathcal{S}_{j}$ defined as above by $x_{j}:=x_{a_{ \pm}}$if $\mathcal{S}_{j} \equiv \mathcal{S}_{a_{ \pm}}$) which indeed implies (using $x_{2}-x_{1} \in \overline{\mathcal{S}_{2}}$ and $x_{2}+x_{1} \in \mathcal{S}_{2}$ in the second inclusion)

$$
\begin{aligned}
e_{n}\left(c_{1}+b_{1}+\mathcal{S}_{1}\right)=c_{1}+b_{1}+\mathcal{S}_{1}-n x_{1} & \subset\left(c_{2}+b_{2}+\mathcal{S}_{2}-n x_{1}\right) \vee\left(c_{2}-b_{2}-\mathcal{S}_{2}-n x_{1}\right) \\
& \subset\left(c_{2}+b_{2}+\mathcal{S}_{2}-n x_{2}\right) \vee\left(c_{2}-b_{2}-\mathcal{S}_{2}+n x_{2}\right) \\
& =e_{n}\left(c_{2}+b_{2}+\mathcal{S}_{2}\right) \vee e_{n}\left(c_{2}-b_{2}-\mathcal{S}_{2}\right) .
\end{aligned}
$$

Since $e_{n}\left(c_{1}-\left(b_{1}+\mathcal{S}_{1}\right)\right)$ can be treated similarly, this proves the isotony of $\tilde{e}_{n}$.

\subsection{Composition of Sectors}

The aim of the present section is to recall how the categories $\Delta(X)$ can be equipped with a monoidal structure. That this is possible relies on the properties of the homomorphisms $\rho$ listed in Lemma 3.2 which reflect their spacetime localisation. The main idea is best illustrated in the simplest example when the set $\mathcal{X}$ is directed. In this case, Lemma 3.2 implies that each $\rho \in \Delta$ can be extended to the $\mathrm{C}^{*}$-algebra $\mathcal{E}:={\overline{\bigcup \mathfrak{A}_{I}(X)^{\prime \prime}}}^{\|\cdot\|}$; hence $\Delta$ can be regarded in a natural way as a full subcategory of End $\mathcal{E}$. The latter is a monoidal $\mathrm{C}^{*}$-category, and because each $\rho \in \Delta$ is transportable, $\Delta$ is readily seen to be closed under the monoidal product of End $\mathcal{E}$, thus becoming a monoidal $\mathrm{C}^{*}$-category on its own.

The situation is more complicated in the cases corresponding to non-compactly localised charges. The reason is that all the objects $\rho \in \Delta$ cannot be regarded in a natural way as endomorphisms of some fixed $\mathrm{C}^{*}$-algebra which also contains the morphisms of $\Delta$. It therefore constituted a considerable progress that Buchholz and Fredenhagen [9] found a way to extend the formalism to this more general situation. (See also [30], where a slightly different approach has been chosen.) From the categorical point of view, the main idea is to endow each $\Delta(X)$ with a monoidal structure by embedding it into the monoidal $\mathrm{C}^{*}$-category $\operatorname{End} \mathcal{E}(X)$, where $\mathcal{E}(X)$ is a suitable $\mathrm{C}^{*}$-algebra which may depend on $X$, and by showing that the image of this inclusion is closed under the monoidal product of End $\mathcal{E}(X)$. Thus in this case, each $\Delta(X)$ becomes a monoidal $\mathrm{C}^{*}$-category, but $\Delta$ does not.

In [9], certain auxiliary cones $\mathcal{S}_{a}$ play a technically important role, leading to auxiliary algebras $\mathcal{B}^{\mathcal{S}_{a}}$. Here the corresponding role is played by the choice of an enlargement in the sense of assumption e. More specifically, let us assume that we are given an enlargement $\left(e_{n}\right)_{n \in \mathbb{N}}$. We will write $X^{(n)}:=e_{n}(X)$ in the sequel, thus suppressing an explicit notational reference to the chosen enlargement. This will be justified at the very end of this section (Prop. 3.10) where it is verified that the monoidal structure to be put on $\Delta(X)$ actually

is independent of that enlargement. Now let $\mathcal{E}(X):=\overline{\bigcup_{n} \mathfrak{A}_{I}\left(X^{(n)}\right)^{\prime \prime}}{ }^{\|\cdot\|}$. The properties of an enlargement imply that $X \longmapsto \mathcal{E}(X)$ is an isotonous net of $\mathrm{C}^{*}$-algebras which fulfils

$$
\mathfrak{A}_{I} \subset \mathcal{E}(X) \subset \mathcal{B}\left(\mathcal{H}_{I}\right) \quad \text { and } \quad \mathfrak{A}_{I}(X)^{\prime \prime} \subset \mathcal{E}(X) .
$$

Moreover one can show directly that $\rho\left(\mathfrak{A}_{I}\right) \subset \mathcal{E}(X)$ if $\rho \in \operatorname{Hom}\left(\mathfrak{A}_{I}, \mathcal{B}\left(\mathcal{H}_{I}\right)\right)$ is localised in $X$. The first part of the following lemma uses the transportability of $\rho$ to strengthen this statement. 


\section{Lemma 3.4}

i. For each object $\rho \in \Delta(X)$, there is a unique endomorphism $\rho^{X}$ of the $C^{*}$-algebra $\mathcal{E}(X)$ which extends $\rho$ and is normal on every subalgebra $\mathfrak{A}_{I}\left(X^{(n)}\right)^{\prime \prime}$.

ii. If $(\tau, t, \sigma)$ is a morphism (from $\sigma$ to $\tau$ ) in $\Delta(X)$, then $\left(\tau^{X}, t, \sigma^{X}\right)$ is a morphism in End $\mathcal{E}(X)$, and any morphism from $\sigma^{X}$ to $\tau^{X}$ is of this form.

Proof: i. Since $\rho$ is transportable, it is unitarily implementable on each subalgebra $\mathfrak{A}_{I}\left(X^{(n)}\right) \subset \mathcal{E}(X)$, i.e., $\left.\rho\right|_{\mathfrak{A}_{I}\left(X^{(n)}\right)}=\operatorname{Ad} w_{n}$ for some unitary $w_{n} \in \mathcal{B}\left(\mathcal{H}_{I}\right)$. Normality is therefore established by setting $\left.\rho^{X}\right|_{\mathfrak{A}_{I}\left(X^{(n)}\right)^{\prime \prime}}:=\mathrm{Ad} w_{n}$. This definition does not, in fact, depend on the choice of $w_{n}$ since, if $\tilde{w}_{n}$ were another unitary satisfying $\left.\rho\right|_{\mathfrak{A}_{I}\left(X^{(n)}\right)}=\operatorname{Ad} \tilde{w}_{n}$, then one would have $\tilde{w}_{n}^{*} w_{n} \in \mathfrak{A}_{I}\left(X^{(n)}\right)^{\prime}$ and hence $\left.\rho^{X}\right|_{\mathfrak{A}_{I}\left(X^{(n)}\right)^{\prime \prime}}=\operatorname{Ad} \tilde{w}_{n}$. One therefore has $\rho^{X}\left(\mathfrak{A}_{I}\left(X^{(n)}\right)^{\prime \prime}\right)=\rho\left(\mathfrak{A}_{I}\left(X^{(n)}\right)\right)^{\prime \prime}$. Since $\rho$ is localised in $X^{(n)} \supset X$, Lemma 3.2, i implies $\rho^{X}\left(\mathfrak{A}_{I}\left(X^{(n)}\right)^{\prime \prime}\right) \subset \mathfrak{A}_{I}\left(X^{(n)}\right)^{\prime \prime}$ for all $n$. It then follows by norm continuity that $\rho^{X}$ is an endomorphism of $\mathcal{E}(X)$.

ii. If $\sigma, \tau \in \Delta(X)$ then the operator $t$ is contained in $\mathfrak{A}_{I}(X)^{\prime \prime} \subset \mathcal{E}(X)$ by Lemma 3.2, ii and satisfies $t \sigma(a)=\tau(a) t$ for all $a \in \mathfrak{A}_{I}$. But then one has $t \sigma^{X}(a)=\tau^{X}(a) t$ for all $a \in \mathfrak{A}_{I}\left(X^{(n)}\right)^{\prime \prime}$ (and any $n$ ) by weak continuity and hence also for all $a \in \mathcal{E}(X)$ by norm continuity. The converse statement is obvious.

In other words, $\Delta(X)$ can be mapped onto a full subcategory of End $\mathcal{E}(X)$. Composing the inclusion functor $\Delta(X) \hookrightarrow \operatorname{End} \mathcal{E}(X)$ with the monoidal product $(\cdot, \otimes)$ of $\operatorname{End} \mathcal{E}(X)$ and regarding the result as lying in $\operatorname{Hom}\left(\mathfrak{A}_{I}, \mathcal{B}\left(\mathcal{H}_{I}\right)\right)$, one thus has a bilinear *-functor

$$
m_{X}: \Delta(X) \times \Delta(X) \longrightarrow \operatorname{Hom}\left(\mathfrak{A}_{I}, \mathcal{B}\left(\mathcal{H}_{I}\right)\right) .
$$

Writing $\dot{X}_{X}$ on the objects and $\underset{X}{\otimes}$ on the morphisms, we can recast this definition of $m_{X}$ as

$$
\begin{aligned}
\rho_{1} \dot{X}_{X} \rho_{2} & :=\left.\left(\rho_{1}^{X} \cdot \rho_{2}^{X}\right)\right|_{\mathfrak{A}_{I}} \\
\left(\tau_{1}, t_{1}, \sigma_{1}\right)_{X}^{\otimes}\left(\tau_{2}, t_{2}, \sigma_{2}\right) & :=\left(\tau_{1} \dot{\chi}_{X}, t_{1} \sigma_{1}^{X}\left(t_{2}\right), \sigma_{1} \dot{X}_{X} \sigma_{2}\right) .
\end{aligned}
$$

Notice that due to $\iota^{X}=\operatorname{id}_{\mathcal{E}(X)}$, the neutral elements of $\dot{X}_{X}$ and ${ }_{X}^{\otimes}$ are $\iota$ and $\mathbf{1}_{\iota}$.

(Here, $\Delta(X) \times \Delta(X)$ denotes the cartesian product of $\Delta(X)$ with itself, equipped with the componentwise *-operation. It suffices to consider each set of morphisms $I\left(\sigma_{1}, \tau_{1}\right) \times I\left(\sigma_{2}, \tau_{2}\right)$ of this category as the cartesian product of two complex vector spaces. The above-mentioned properties of $m_{X}$ are then self-explanatory, and their verification is straightforward.)

In order to show that $\Delta(X)$ equipped with $m_{X}$ as the product is a monoidal subcategory of $\operatorname{End} \mathcal{E}(X)$, it has first of all to be shown that the image of $m_{X}$ is contained in $\Delta(X)$. As a step towards that, it is convenient to check that the family $\left(m_{X}\right)_{X \in \mathcal{X}}$ is compatible with the inclusions $X_{1} \subset X_{2}$ :

Lemma 3.5 Let $X_{1} \subset X_{2}$. For any $\rho \in \Delta\left(X_{1}\right) \subset \Delta\left(X_{2}\right)$, one then has $\left.\rho^{X_{2}}\right|_{\mathcal{E}\left(X_{1}\right)}=\rho^{X_{1}}$.

Proof: Since $\rho^{X_{2}}$ is normal on any $\mathfrak{A}_{I}\left(X_{2}^{(n)}\right)^{\prime \prime}$ and $X_{1}^{(n)} \subset X_{2}^{(n)}$ by the isotony of each $e_{n}$, it follows that $\left.\rho^{X_{2}}\right|_{\mathcal{E}\left(X_{1}\right)}$ is an extension of $\rho$ which is normal on any subalgebra $\mathfrak{A}_{I}\left(X_{1}^{(n)}\right)^{\prime \prime}$. It must therefore coincide with $\rho^{X_{1}}$. 
Thus as a consequence the operations $\underset{X_{2}}{\dot{2}}$ and $\underset{X_{2}}{\otimes}$ restrict to ${\dot{X_{1}}}^{\cdot}$ and $\underset{X_{1}}{\otimes}$, respectively, and one may reformulate this by saying that

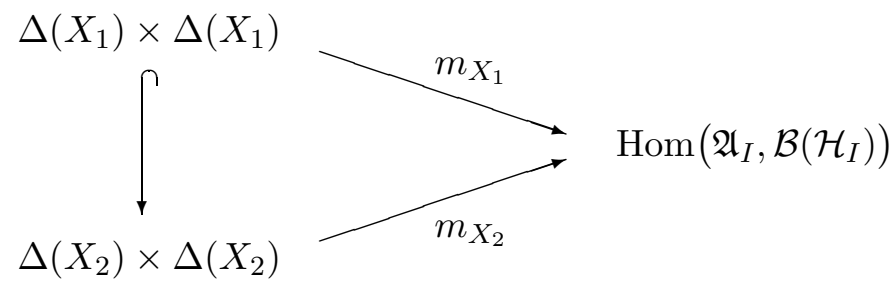

is a commutative diagram (in the category of all categories equipped with a $\mathbb{C}$-linear structure and an antilinear ${ }^{*}$-operation on the morphisms, say.)

We are now ready for the proof of

Lemma 3.6 Let $\rho, \sigma \in \Delta(X)$. Then $\rho_{X} \sigma \in \Delta(X)$, and $\underset{X}{(\rho \cdot \sigma)^{X}}=\rho^{X} \cdot \sigma^{X}$.

Proof: It has to be shown that $\rho_{X} \sigma$ is localised in $X$ and transportable. The former is true due to $\left.(\rho \cdot \sigma)\right|_{\mathfrak{A}_{I}\left(X^{\prime}\right)}=\left.\rho^{X} \cdot \sigma^{X}\right|_{\mathfrak{A}_{I}\left(X^{\prime}\right)}=\left.\rho^{X}\right|_{\mathfrak{A}_{I}\left(X^{\prime}\right)}=\left.\mathrm{id}\right|_{\mathfrak{A}_{I}\left(X^{\prime}\right)}$, since both $\rho$ and $\sigma$ are localised in $X$. To see the transportability, choose some $X_{0} \in \mathcal{X}$. By assumption $\mathbf{c 1}, \mathcal{X}$ is connected, so there exists in $\mathcal{X}$ a finite path $X_{0} \supset X_{1} \subset X_{2} \supset X_{3} \subset \cdots \supset X_{2 N-1} \subset$ $X_{2 N}=X$. As $\rho$ and $\sigma$ are transportable, there exist $\rho_{j}, \sigma_{j} \in \Delta\left(X_{j}\right), \rho_{j} \cong \rho, \sigma_{j} \cong \sigma$ for $j=0, \ldots, 2 N$. For any odd $j$, one then has $X_{j} \subset X_{j \pm 1}, \rho_{j} \cong \rho_{j \pm 1}, \sigma_{j} \cong \sigma_{j \pm 1}$ and hence $\rho_{j} \dot{X}_{j} \sigma_{j}=\rho_{j} \underset{X_{j \pm 1}}{\cdot} \sigma_{j} \cong \rho_{j \pm 1} \cdot \dot{X_{j \pm 1}} \sigma_{j \pm 1}$, where the equality holds because of Lemma 3.5, and

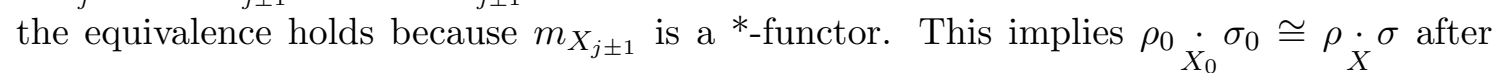
finitely many steps. Since $X_{0}$ was arbitrary, this proves $\rho_{X} \sigma \in \Delta(X)$. Therefore $\left(\rho_{X} \sigma\right)^{X}$ is a well-defined endomorphism of $\operatorname{End} \mathcal{E}(X)$ which coincides with $\rho^{X} \cdot \sigma^{X}$ by Lemma 3.4, i.

As the subcategory $\Delta(X)$ of $\operatorname{Hom}\left(\mathfrak{A}_{I}, \mathcal{B}\left(\mathcal{H}_{I}\right)\right)$ is full, the image of $m_{X}$ is thus contained in $\Delta(X)$, so we have at this point a bilinear ${ }^{*}$-functor $m_{X}: \Delta(X) \times \Delta(X) \longrightarrow \Delta(X)$ which makes the following diagram commute. (On the level of objects this is due to Lemma 3.6, on the level of morphisms, it is due to the inclusion being trivial.)

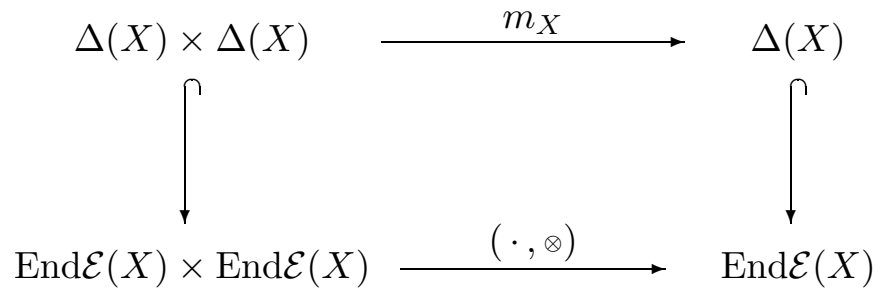

Lemma 3.7 Equipped with $m_{X}: \Delta(X) \times \Delta(X) \longrightarrow \Delta(X)$ as the monoidal product, $\Delta(X)$ is a monoidal $C^{*}$-category. The monoidal unit is the object $\iota \in \Delta(X)$.

Proof: From the above discussion, it follows that $m_{X}$ is the restriction of $(\cdot, \otimes)$ to the $\mathrm{C}^{*}$-subcategory $\Delta(X)$. But this already implies the assertion since the monoidal unit $\operatorname{id}_{\mathcal{E}(X)}=\iota^{X}$ is in the image of the inclusion $\Delta(X) \hookrightarrow \operatorname{End} \mathcal{E}(X)$. 
From now on, we will abbreviate the notation by omitting the subscript $X$ on the symbols for the monoidal products, but it should be emphasised that these operations are only defined within a fixed category $\Delta(X)$.

Remark: It might happen that, given two endomorphisms $\rho, \sigma \in \Delta$, the set of regions $\{X \in \mathcal{X} \mid \rho, \sigma \in \Delta(X)\}$ is nonempty but not connected. In this case, $\rho \cdot \sigma$ could possibly depend on the choice of a connected component of this set. Examples for the underlying geometric situation can easily be obtained if $\mathcal{X}$ is, e.g., the set of all opposite spacelike cones: just choose $\rho \in \Delta\left(X_{1}\right)$ and $\sigma \in \Delta\left(X_{2}\right)$, where $X_{1}$ and $X_{2}$ are sufficiently small neighbourhoods of two spacelike lines at time zero, say.

We notice a continuity property of the product $m_{X}$ on $\Delta(X)$ considered as a $\mathrm{W}^{*}$ category, namely:

Lemma 3.8 Let $\rho, \sigma, \tau \in \Delta(X)$. Then the maps

$$
I(\sigma, \tau) \longrightarrow I(\sigma \cdot \rho, \tau \cdot \rho): t \longmapsto t \otimes \mathbf{1}_{\rho} \quad \text { and } \quad I(\sigma, \tau) \longrightarrow I(\rho \cdot \sigma, \rho \cdot \tau): t \longmapsto \mathbf{1}_{\rho} \otimes t
$$

are $w^{*}$-continuous.

Proof: Considered as maps on the set $I(\sigma, \tau) \subset \mathfrak{A}_{I}(X)^{\prime \prime} \subset \mathcal{B}\left(\mathcal{H}_{I}\right)$, the above maps read $t \longmapsto t$ and $t \longmapsto \rho^{X}(t)$, respectively. Since the $\mathrm{w}^{*}$-topologies on the sets of morphisms are induced from the $\mathrm{w}^{*}$-topology on $\mathcal{B}\left(\mathcal{H}_{I}\right)$, the assertion is trivial for the first of these maps and follows for the second one from the normality of $\rho^{X}$ on $\mathfrak{A}_{I}(X)^{\prime \prime}$, cf. Lemma 3.4.

In Appendix A, we have chosen to include this continuity into our definition of a monoidal $\mathrm{W}^{*}$-category, and we decided to coin the term " $\mathrm{rW}^{*}$-category" for a monoidal $\mathrm{W}^{*}$-category with irreducible monoidal unit which is closed under subobjects and finite direct sums. With these notions, the state of affairs can be summarised as follows (cf. Thm. 4.11 of $[2.9])$ :

\section{Proposition 3.9}

i. The map $X \longmapsto \Delta(X)$ is an isotonous net of $r W^{*}$-categories.

ii. For $X_{1} \subset X_{2}$, the inclusion $\Delta\left(X_{1}\right) \hookrightarrow \Delta\left(X_{2}\right)$ is a full monoidal $W^{*}$-functor, and $\Delta\left(X_{1}\right)$ is equivalent to $\Delta\left(X_{2}\right)$.

We conclude this section with the proof that the monoidal product on $\Delta(X)$ does not depend on the chosen enlargement of $\mathcal{X}$. To this end, let $\left(e_{n}^{(j)}\right)_{n \in \mathbb{N}}(j=1,2)$ be two enlargements of $\mathcal{X}$, put $X^{(j, n)}:=e_{n}^{(j)}(X)$, denote with $\mathcal{E}^{(j)}(X)$ the norm closures of $\mathcal{E}_{0}^{(j)}(X):=\bigcup_{n} \mathfrak{A}_{I}\left(X^{(j, n)}\right)^{\prime \prime}$ and let, for any object $\rho \in \Delta(X), \rho^{(j, X)} \in \operatorname{End}^{(j)}(X)$ be the corresponding endomorphism of the $\mathrm{C}^{*}$-algebra $\mathcal{E}^{(j)}(X)$, cf. Lemma 3.4. Finally, let $\mathcal{E}^{(1,2)}(X):={\overline{\mathcal{E}_{0}^{(1)}(X) \cap \mathcal{E}_{0}^{(2)}(X)}}^{\|\cdot\|} \subset \mathcal{E}^{(1)}(X) \cap \mathcal{E}^{(2)}(X)$. With these notations, one can prove:

Proposition 3.10 Let $X \in \mathcal{X}$. Then:

i. $\rho^{(1, X)}$ and $\rho^{(2, X)}$ define the same endomorphism of $\mathcal{E}^{(1,2)}(X)$.

ii. The monoidal product on $\Delta(X)$ does not depend on the chosen enlargement of $\mathcal{X}$.

Proof: i. For each $j, n$ choose some $\tilde{X}_{n}^{(j)} \times X^{(j, n)}$, some $\tilde{\rho}_{n}^{(j)} \in \Delta\left(\tilde{X}_{n}^{(j)}\right)$ equivalent to $\rho$ and a unitary intertwiner $w_{n}^{(j)} \in \mathcal{B}\left(\mathcal{H}_{I}\right)$ from $\tilde{\rho}_{n}^{(j)}$ to $\rho$. Then $\left.\rho^{(j, X)}\right|_{\mathfrak{A}_{I}\left(X^{(j, n)}\right)^{\prime \prime}}=\operatorname{Ad} w_{n}^{(j)}$. Now 
$w_{n}^{(2) *} w_{n}^{(1)}$ intertwines from $\tilde{\rho}_{n}^{(1)}$ to $\tilde{\rho}_{n}^{(2)}$, hence it is contained in $\left(\mathfrak{A}_{I}\left(\tilde{X}_{n}^{(1) \prime}\right) \cap \mathfrak{A}_{I}\left(\tilde{X}_{n}^{(2) \prime}\right)\right)^{\prime}$ (by the localisation of $\tilde{\rho}_{n}^{(j)}$ ), which is a subalgebra of $\left(\mathfrak{A}_{I}\left(X^{(1, n)}\right)^{\prime \prime} \cap \mathfrak{A}_{I}\left(X^{(2, n)}\right)^{\prime \prime}\right)^{\prime}$ (due to the choice of $\left.\tilde{X}_{n}^{(j)}\right)$. Therefore one has for any $a \in \mathfrak{A}_{I}\left(X^{(1, n)}\right)^{\prime \prime} \cap \mathfrak{A}_{I}\left(X^{(2, n)}\right)^{\prime \prime}$ the relation $w_{n}^{(2) *} w_{n}^{(1)} a=a w_{n}^{(2) *} w_{n}^{(1)}$, i.e., $\rho^{(1, X)}(a)=\rho^{(2, X)}(a)$. As $n$ was arbitrary, this means that $\rho^{(1, X)}$ and $\rho^{(2, X)}$ agree on $\bigcup_{n}\left(\mathfrak{A}_{I}\left(X^{(1, n)}\right)^{\prime \prime} \cap \mathfrak{A}_{I}\left(X^{(2, n)}\right)^{\prime \prime}\right)=\mathcal{E}_{0}^{(1)}(X) \cap \mathcal{E}_{0}^{(2)}(X)$. Because each $\rho^{(j, X)}$ maps $\mathcal{E}_{0}^{(j)}(X)$ into itself, this implies that $\rho^{(1, X)}$ and $\rho^{(2, X)}$ map $\mathcal{E}_{0}^{(1)}(X) \cap \mathcal{E}_{0}^{(2)}(X)$ into itself; by norm continuity, this yields Part i.

ii. For each double cone $\mathcal{O}$, one has $\mathfrak{A}_{I}(\mathcal{O}) \subset \mathcal{E}_{0}^{(1)}(X) \cap \mathcal{E}_{0}^{(2)}(X)$, whence $\mathfrak{A}_{I} \subset \mathcal{E}^{(1,2)}(X)$, cf. the remark after assumption e on p. 22. Therefore Part i implies that the product $\sigma \cdot \tau=\left.\left(\sigma^{(j, X)} \cdot \tau^{(j, X)}\right)\right|_{\mathfrak{A}_{I}}$ of two objects in $\Delta(X)$ does not depend on the enlargement. As to the product of morphisms, recall that $t \in \mathfrak{A}_{I}(X)^{\prime \prime}$ whenever $t$ is a morphism in $\Delta(X)$. Hence the normality of the extension of $\rho$ to $\mathfrak{A}_{I}(X)^{\prime \prime}$ yields $\rho^{(1, X)}(t)=\rho^{(2, X)}(t)$.

\subsection{The Symmetry}

In this section, we will show that each monoidal $\mathrm{C}^{*}$-category $\Delta(X), X \in \mathcal{X}$ can be equipped in a natural way with a symmetry $\epsilon^{X}$ (cf. Appendix A.2). This will be done by invoking the slightly more abstract result of Lemma A.2, the premises of which we thus have to check first. We begin with a classical lemma establishing a form of local commutativity of the net $X \longmapsto \Delta(X)$ with respect to the monoidal product:

Lemma 3.11 Let $\left(X_{1}, X_{2}\right) \in \mathcal{X}_{\mathbf{x}}^{(2)}(X)$.

i. If $\rho_{j} \in \Delta\left(X_{j}\right), j=1,2$, then $\rho_{1} \cdot \rho_{2}=\rho_{2} \cdot \rho_{1}$.

ii. If $\sigma_{j}, \tau_{j} \in \Delta\left(X_{j}\right)$ and $t_{j}: \sigma_{j} \rightarrow \tau_{j}$, then $t_{1} \otimes t_{2}=t_{2} \otimes t_{1}$.

Proof: i. We will show $\rho_{1}^{X} \cdot \rho_{2}^{X}=\rho_{2}^{X} \cdot \rho_{1}^{X}$ on all of $\mathcal{E}(X)$. For any $n \in \mathbb{N}$, one has by definition $\left.\rho_{j}^{X}\right|_{\mathfrak{A}_{I}\left(X^{(n)}\right)^{\prime \prime}}=\operatorname{Ad} w_{j, n}$ for suitable unitaries $w_{j, n}$ which satisfy $w_{j, n} \in \mathfrak{A}_{I}\left(X_{j}^{\prime}\right)^{\prime}=\mathfrak{A}_{I}\left(X_{j}\right)^{\prime \prime}$ because of duality and the localisation of $\rho_{j}$. Locality now implies $\left[w_{1, n}, w_{2, n}\right]=0$ and hence $\rho_{1}^{X} \cdot \rho_{2}^{X}=\rho_{2}^{X} \cdot \rho_{1}^{X}$ on $\mathfrak{A}_{I}\left(X^{(n)}\right)^{\prime \prime}$. Since $n$ was arbitrary, this implies the assertion by norm continuity.

ii. By Part i we have $\sigma_{1} \cdot \sigma_{2}=\sigma_{2} \cdot \sigma_{1}$ and $\tau_{1} \cdot \tau_{2}=\tau_{2} \cdot \tau_{1}$, and it only has to be shown that $t_{1} \sigma_{1}^{X}\left(t_{2}\right)=t_{2} \sigma_{2}^{X}\left(t_{1}\right)$. Now $t_{j} \in \mathfrak{A}_{I}\left(X_{j}\right)^{\prime \prime}$ and therefore $\left[t_{1}, t_{2}\right]=0$ by locality, so the assertion follows from the relations $\sigma_{1}^{X}\left(t_{2}\right)=t_{2}$ and $\sigma_{2}^{X}\left(t_{1}\right)=t_{1}$ which in turn can be seen as follows: $X_{1} \subset X_{2}^{\prime}$ implies $\left.\sigma_{1}\right|_{\mathfrak{A}_{I}\left(X_{2}\right)}=\mathrm{id}$, hence $\left.\sigma_{1}^{X}\right|_{\mathfrak{A}_{I}\left(X_{2}\right)^{\prime \prime}}=\mathrm{id}$ and thus $\sigma_{1}^{X}\left(t_{2}\right)=t_{2}$; similarly, one obtains $\sigma_{2}^{X}\left(t_{1}\right)=t_{1}$.

Let us now introduce on the set of objects of each $\Delta(X)$ the relation

$$
\rho_{1} \perp \rho_{2}: \Longleftrightarrow \text { there exists }\left(X_{1}, X_{2}\right) \in \mathcal{X}_{\times}^{(2)}(X) \text { such that } \rho_{j} \in \Delta\left(X_{j}\right) .
$$

With this notation and with the assumption $\mathbf{c 2}$ as only new ingredient, the previous lemma can be strengthened:

Lemma 3.12 Let $t_{j}: \sigma_{j} \rightarrow \tau_{j}(j=1,2)$ be morphisms in $\Delta(X)$ such that $\sigma_{1} \perp \sigma_{2}$ and $\tau_{1} \perp \tau_{2}$. Then $t_{1} \otimes t_{2}=t_{2} \otimes t_{1}$. 
Proof: By the definition of $\perp$, there exist pairs $\left(X_{1}, X_{2}\right)$ and $\left(\tilde{X}_{1}, \tilde{X}_{2}\right)$ in $\mathcal{X}_{\mathbf{x}}^{(2)}(X)$ such that $\sigma_{j} \in \Delta\left(X_{j}\right)$ and $\tau_{j} \in \Delta\left(\tilde{X}_{j}\right)$. By assumption c2 $\mathcal{X}_{\times}^{(2)}(X)$ is connected; it is therefore possible to choose a path $\left(Y_{1, \nu}, Y_{2, \nu}\right)_{\nu=0, \ldots, N}$ in $\mathcal{X}_{\mathbf{x}}^{(2)}(X)$ going from $\left(Y_{1,0}, Y_{2,0}\right)=\left(X_{1}, X_{2}\right)$ to $\left(Y_{1, N}, Y_{2, N}\right)=\left(\tilde{X}_{1}, \tilde{X}_{2}\right)$. Then there exist objects $\rho_{j, \nu} \in \Delta\left(Y_{j, \nu}\right), \nu=1, \ldots, N$ equivalent to $\rho_{j, 0}:=\sigma_{j}$ and unitaries $v_{j, \nu}: \rho_{j, \nu-1} \rightarrow \rho_{j, \nu}$ such that $t_{j}$ can be factorised as $t_{j}=\tilde{t}_{j} \circ v_{j, N} \circ \cdots \circ v_{j, 2} \circ v_{j, 1}$. This means $t_{1} \otimes t_{2}=\tilde{t}_{1} \otimes \tilde{t}_{2} \circ v_{1, N} \otimes v_{2, N} \circ \cdots \circ v_{1,1} \otimes v_{2,1}$. Part ii of Lemma 3.11 can now be applied to each of the $N+1$ terms of this product since $\left(Y_{1, \nu} \cup Y_{1, \nu-1}, Y_{2, \nu} \cup Y_{2, \nu-1}\right) \in \mathcal{X}_{\mathbf{x}}^{(2)}(X)$ for each $\nu=1, \ldots, N$ as a consequence of the definition of a path in $\mathcal{X}_{\times}^{(2)}(X)$. But then $t_{1} \otimes t_{2}=\tilde{t}_{2} \otimes \tilde{t}_{1} \circ v_{2, N} \otimes v_{1, N} \circ \cdots \circ v_{2,1} \otimes v_{1,1}=t_{2} \otimes t_{1}$, which was the assertion.

After these preparations, Lemma A.2 can be applied to each $(\Delta(X), \perp)$, and we thus obtain:

\section{Proposition 3.13}

i. Each category $\Delta(X)$ possesses a unique symmetry $\epsilon^{X}$ with the property that $\epsilon^{X}\left(\rho_{1}, \rho_{2}\right)=\mathbf{1}_{\rho_{1} \rho_{2}}$ if $\rho_{1} \perp \rho_{2}$.

ii. $X \longmapsto\left(\Delta(X), \epsilon^{X}\right)$ is an isotonous net of symmetric monoidal $C^{*}$-categories.

Proof: Part i follows from the previous discussion; note in particular that the third of the conditions on $\perp$ listed before Lemma A.2 is fulfilled by any pair $\Delta\left(X_{1}\right), \Delta\left(X_{2}\right)$, where $\left(X_{1}, X_{2}\right) \in \mathcal{X}_{\mathbf{\times}}^{(2)}(X)$. For Part ii, it has to be shown that $\epsilon^{X_{2}}$ restricts to $\epsilon^{X_{1}}$ if $X_{1} \subset X_{2}$. But this is obvious since both symmetries have the property that $\epsilon^{X_{j}}\left(\rho_{1}, \rho_{2}\right)=\mathbf{1}_{\rho_{1} \rho_{2}}$ if $\rho_{1}, \rho_{2} \in \Delta\left(X_{1}\right)$ fulfil $\rho_{1} \perp \rho_{2}$ which fixes them uniquely.

We will omit the superscript $X$ on $\epsilon^{X}$ in the sequel. Again, it should be kept in mind that a morphism $\epsilon\left(\rho_{1}, \rho_{2}\right)$ is defined with respect to some $X \in \mathcal{X}$ such that $\rho_{1}, \rho_{2} \in \Delta(X)$.

\subsection{Conjugates and Left Inverses}

In Appendix A.3, we review the classification of statistics and the existence of conjugates in a symmetric $\mathrm{rW}^{*}$-category. The analysis presented there is very general and rests on the following two assumptions:

- each object has a left inverse;

- the simple objects (i.e., those objects $\gamma$ with statistical dimension $d(\gamma)=1$ ) possess conjugates.

We will now verify these assumptions in the case of the categories $\Delta(X)$. Since the above notions are intimately related to the monoidal product, it is not surprising that the verification of these items involves detailed information on how this product was defined. Accordingly, it will be necessary to recall the steps which led to the definition of the functors $m_{X}$ and, in particular, the proof (and not merely the statement) of Lemma 3.4.

The question of left inverses is handled as follows [7].

Lemma 3.14 Let $X \in \mathcal{X}$ and $\rho \in \Delta(X)$. Then:

i. There exists a positive linear map $\Phi: \mathcal{B}\left(\mathcal{H}_{I}\right) \longrightarrow \mathcal{B}\left(\mathcal{H}_{I}\right)$ satisfying $\Phi(\mathbf{1})=\mathbf{1}$ and $\Phi\left(\rho^{X}\left(a_{1}\right) b \rho^{X}\left(a_{2}\right)\right)=a_{1} \Phi(b) a_{2}$ for all $a_{1}, a_{2} \in \mathcal{E}(X), b \in \mathcal{B}\left(\mathcal{H}_{I}\right)$. 


\section{ii. The set $\operatorname{LI}(\rho)$ of left inverses of $\rho$ is nonempty.}

Proof: i. In the proof of Lemma 3.4 the homomorphism $\rho^{X}: \mathcal{E}(X) \longrightarrow \mathcal{B}\left(\mathcal{H}_{I}\right)$ was defined by $\left.\rho^{X}\right|_{\mathfrak{A}_{I}\left(X^{(n)}\right)^{\prime \prime}}=\operatorname{Ad} w_{n}$ with a suitable sequence $\left(w_{n}\right)_{n \in \mathbb{N}}$ of unitaries in $\mathcal{B}\left(\mathcal{H}_{I}\right)$. Now consider the maps $\mathrm{Ad} w_{n}^{*}$, which are positive elements of the unit ball $B_{1}$ of the Banach space $B:=\mathcal{B}\left(\mathcal{B}\left(\mathcal{H}_{I}\right), \mathcal{B}\left(\mathcal{H}_{I}\right)\right)$ of bounded linear maps from $\mathcal{B}\left(\mathcal{H}_{I}\right)$ to itself. As the unit ball of $\mathcal{B}\left(\mathcal{H}_{I}\right)$ is compact in the weak operator topology, it follows that $B_{1}$ is compact when equipped with the pointwise weak operator topology, cf. e.g. [3T]. Hence there exist limit points of $\left\{\operatorname{Ad} w_{n}^{*} \mid n \in \mathbb{N}\right\}$, and any such limit point $\Phi$ is a (positive and unit-preserving) element of $B_{1}$. Due to the positivity, the equation asserted in i need only be checked for the case $a_{2}=1$. Now if $a \in \mathfrak{A}_{I}\left(X^{\left(n_{0}\right)}\right)^{\prime \prime}$ for some $n_{0} \in \mathbb{N}$, one has $\rho^{X}(a)=\operatorname{Ad} w_{n}(a)$ for all $n \geq n_{0}$ and hence $\Phi\left(\rho^{X}(a) b\right)=\mathrm{w}-\lim w_{n}^{*} \rho^{X}(a) b w_{n}=\mathrm{w}-\lim a w_{n}^{*} b w_{n}=a \Phi(b)$ (the limit being taken on some appropriate subnet of $\mathbb{N})$. As $n_{0}$ was arbitrary, this implies $\Phi\left(\rho^{X}(a) b\right)=a \Phi(b)$ for any $a \in \mathcal{E}(X)$ by norm continuity.

ii. A left inverse $\varphi=\left(\varphi_{\sigma, \tau}\right)_{\sigma, \tau \in \Delta(X)}$ of $\rho$ can now easily be obtained from the map $\Phi$ by letting $\varphi_{\sigma, \tau}((\rho \tau, t, \rho \sigma)):=(\tau, \Phi(t), \sigma)$. First, it has to be checked that this defines a map from $I(\rho \sigma, \rho \tau)$ to $I(\sigma, \tau)$. But if $t: \rho \sigma \rightarrow \rho \tau$ in $\Delta(X)$, then $t: \rho^{X} \cdot \sigma^{X} \rightarrow \rho^{X} \cdot \tau^{X}$ in $\operatorname{End} \mathcal{E}(X)$ by Lemma 3.4, ii. In particular, $t \rho^{X}(\sigma(a))=\rho^{X}(\tau(a)) t$ for all $a \in \mathfrak{A}_{I}$, which implies by Part i that $\Phi(t) \in \mathcal{B}\left(\mathcal{H}_{I}\right)$ satisfies $\Phi(t) \sigma(a)=\tau(a) \Phi(t)$, i.e., $\Phi(t) \in I(\sigma, \tau)$. Second, it has to be verified that the family $\left(\varphi_{\sigma, \tau}\right)_{\sigma, \tau \in \Delta(X)}$ of linear maps is indeed a left inverse of $\rho$. As the corresponding computations are elementary, we leave them to the reader.

Remark: Although $\Delta(X)$ is embedded into $\operatorname{End} \mathcal{E}(X)$ as a full subcategory, it does not follow automatically that the image $\rho^{X} \in \operatorname{End} \mathcal{E}(X)$ of some $\rho \in \Delta(X)$ possesses a left inverse. The reason is that $\Phi$ might not map $\mathcal{E}(X)$ to itself; as a consequence, there are no candidates for maps $\varphi_{\tilde{\sigma}, \tilde{\tau}}$ if $\tilde{\sigma}, \tilde{\tau} \in \operatorname{End} \mathcal{E}(X)$ are not in the image of the embedding.

The next item is the existence of conjugates. It relies on the assumption $\mathbf{c 3}$, which enters into Lemma 3.15. This lemma involves the definition of the $\mathrm{C}^{*}$-algebras $\mathcal{E}(X)$ in terms of an enlargement. We try to separate this aspect from those issues (treated in Prop. 3.16) which are based on the full monoidal inclusion $\Delta(X) \subset \operatorname{End} \mathcal{E}(X)$.

Lemma 3.15 Let $\gamma \in \Delta(X)$ satisfy $\epsilon_{\gamma} \in\left\{ \pm \mathbf{1}_{\gamma^{2}}\right\}$. Then $\gamma^{X} \in \operatorname{Aut} \mathcal{E}(X)$.

Proof: The proof of this lemma proceeds in three steps. For the first step, consider a situation where $(X, \tilde{X}) \in \mathcal{X}_{\times}^{(2)}(Y)$ for some $\tilde{X}, Y \in \mathcal{X}$. If $\tilde{\gamma} \in \Delta(\tilde{X})$, then $\epsilon(\gamma, \tilde{\gamma})=\mathbf{1}_{\gamma \tilde{\gamma}}$ and $\epsilon(\gamma, \gamma)=\epsilon_{\gamma}= \pm \mathbf{1}_{\gamma^{2}}$ imply (for any $\left.t: \tilde{\gamma} \rightarrow \gamma\right) \mathbf{1}_{\gamma} \otimes t=\epsilon(\gamma, \tilde{\gamma}) \circ \mathbf{1}_{\gamma} \otimes t=t \otimes \mathbf{1}_{\gamma} \circ \epsilon(\gamma, \gamma)=$ $\pm t \otimes \mathbf{1}_{\gamma}$. For the operators in question, this means $\gamma^{Y}(t)= \pm t$.

In a second step, one shows $\mathfrak{A}_{I}(X) \subset \gamma\left(\mathfrak{A}_{I}(X)\right)^{\prime \prime}$. Assumption c3 implies that one can choose $Z, X_{0} \in \mathcal{X}$ such that $\left(X, X_{0}\right) \in \mathcal{X}_{\times}^{(2)}(Z)$ and that there exist sequences $X_{n}, Y_{n}$ in $\mathcal{X}$ with $X_{n}$ tending to spacelike infinity such that $\left(X_{n}, Z\right) \in \mathcal{X}_{\mathbf{\times}}^{(2)}\left(Y_{n}\right)$. Since $X \subset Z$, one has $\left(X_{n}, X\right) \in \mathcal{X}_{\mathbf{x}}^{(2)}\left(Y_{n}\right)$ for each $n \in \mathbb{N}$, and setting $Y_{0}:=Z$, one has this last relation for $n=0$ also. Now take for each $n \in \mathbb{N}_{0}$ some $\gamma_{n} \in \Delta\left(X_{n}\right)$ equivalent to $\gamma$ and some unitary $v_{n} \in \mathcal{B}\left(\mathcal{H}_{I}\right)$ which intertwines from $\gamma$ to $\gamma_{n}$. Since this is the situation considered in the first step, it follows that $\gamma^{Y_{n}}\left(v_{n}\right)= \pm v_{n}, n \in \mathbb{N}_{0}$. Choosing an arbitrary element $a \in \mathfrak{A}_{I}(X)$, one has $a=\gamma_{n}(a)=v_{n} \gamma(a) v_{n}^{*}=\gamma^{Y_{n}}\left(v_{n} a v_{n}^{*}\right)=\gamma^{Y_{n}}\left(b_{n}\right)$ for each $n \in \mathbb{N}_{0}$, where $b_{n}:=v_{n} a v_{n}^{*} \in \mathfrak{A}_{I}\left(X^{\prime}\right)^{\prime} \vee \mathfrak{A}_{I}\left(X_{n}^{\prime}\right)^{\prime}$. But $\gamma^{Y_{n}}\left(b_{n}\right)=a=\gamma^{Y_{0}}\left(b_{0}\right)=\gamma^{Y_{n}}\left(b_{0}\right)$ 
since $Y_{0} \subset Y_{n}$, implying $b_{n}=b_{0}$ for each $n \in \mathbb{N}$ because $\gamma^{Y_{n}}$ is one-to-one. But this means $b_{0} \in \bigcap_{n}\left(\mathfrak{A}_{I}\left(X^{\prime}\right)^{\prime} \vee \mathfrak{A}_{I}\left(X_{n}^{\prime}\right)^{\prime}\right)=\mathfrak{A}_{I}(X)^{\prime \prime} \vee\left(\bigcap_{n} \mathfrak{A}_{I}\left(X_{n}^{\prime}\right)^{\prime}\right)$. Now $\left(X_{n}\right)_{n \in \mathbb{N}}$ tends to spacelike infinity, so $\mathfrak{A}_{I}(\mathcal{O}) \subset \bigcup_{n} \mathfrak{A}_{I}\left(X_{n}^{\prime}\right)$ for any bounded $\mathcal{O} \subset \mathbb{R}^{1+s}$. Since $\pi_{I}$ is irreducible, this implies $\bigcap_{n} \mathfrak{A}_{I}\left(X_{n}^{\prime}\right)^{\prime}=\mathbb{C} \mathbf{1}$. Therefore $b_{0} \in \mathfrak{A}_{I}(X)^{\prime \prime}$, which means $a=\gamma^{Y_{0}}\left(b_{0}\right)=\gamma^{X}\left(b_{0}\right) \in \gamma^{X}\left(\mathfrak{A}_{I}(X)^{\prime \prime}\right)=\gamma\left(\mathfrak{A}_{I}(X)\right)^{\prime \prime}$. As $a \in \mathfrak{A}_{I}(X)$ was arbitrary, one concludes $\mathfrak{A}_{I}(X) \subset \gamma\left(\mathfrak{A}_{I}(X)\right)^{\prime \prime}$.

In the third step, finally, this result is applied to $\gamma$ considered as an object of $\Delta\left(X^{(n)}\right),\left(X^{(n)}\right)_{n \in \mathbb{N}}$ being the enlargement which generates $\mathcal{E}(X)$. One has $\mathfrak{A}_{I}\left(X^{(n)}\right)^{\prime \prime} \subset$ $\gamma\left(\mathfrak{A}_{I}\left(X^{(n)}\right)\right)^{\prime \prime}=\gamma^{X}\left(\mathfrak{A}_{I}\left(X^{(n)}\right)^{\prime \prime}\right)$, which yields $\mathcal{E}(X) \subset \gamma^{X}(\mathcal{E}(X))$. Since $\gamma^{X}$ is an endomorphisms of $\mathcal{E}(X)$ and preserves the norm, this implies $\gamma^{X} \in \operatorname{Aut} \mathcal{E}(X)$.

Lemma 3.15 essentially amounts to $\mathrm{iii} \Rightarrow \mathrm{i}$ in the following proposition. In contrast to $\mathrm{i} \Rightarrow \mathrm{ii} \Rightarrow \mathrm{iii}$, this implication is specific to the present context. The assertion mainly aimed at, namely that all simple objects have a conjugate, is of course covered by $\mathrm{i} \Rightarrow \mathrm{i}$.

Proposition 3.16 Let $\gamma \in \Delta(X)$. Then the following properties are equivalent:

i. $\gamma$ has a monoidal inverse $\bar{\gamma} \in \Delta(X)$, i.e. $\gamma \cdot \bar{\gamma}=\iota=\bar{\gamma} \cdot \gamma$;

ii. $\gamma$ is simple, i.e. $d(\gamma)=1$;

iii. $\epsilon_{\gamma} \in\left\{ \pm \mathbf{1}_{\gamma^{2}}\right\}$.

Proof: The implications $\mathrm{i} \Rightarrow \mathrm{ii} \Rightarrow$ iii rely on the multiplicativity of the dimension and are valid in any symmetric $\mathrm{rC}^{*}$-category, cf. Appendix A.3. We therefore only prove $\mathrm{iii} \Rightarrow \mathrm{i}$ here. Thus, assume $\epsilon_{\gamma} \in\left\{ \pm \mathbf{1}_{\gamma^{2}}\right\}$. In Lemma 3.15, this has been shown to entail $\gamma^{X} \in \operatorname{Aut} \mathcal{E}(X)$. The assertion i therefore follows if the monoidal inverse of $\gamma^{X}$, viz. $\left(\gamma^{X}\right)^{-1}$, is the image $\bar{\gamma}^{X}$ of some $\bar{\gamma} \in \Delta(X)$. Necessarily, $\bar{\gamma}$ is given by $\left.\left(\gamma^{X}\right)^{-1}\right|_{\mathfrak{A}_{I}} \in \operatorname{Hom}\left(\mathfrak{A}_{I}, \mathcal{B}\left(\mathcal{H}_{I}\right)\right)$, and because of $\left.\gamma^{X}\right|_{\mathfrak{A}_{I}\left(X^{\prime}\right)}=\left.\gamma\right|_{\mathfrak{A}_{I}\left(X^{\prime}\right)}=\operatorname{id}_{\mathfrak{A}_{I}\left(X^{\prime}\right)}, \bar{\gamma}$ is localised in $X$. So what is left is the transportability of $\bar{\gamma}$, which follows from that of $\gamma$ : Let $X_{0} \in \mathcal{X}$ and $\gamma_{0} \in \Delta\left(X_{0}\right)$ be equivalent to $\gamma$. Then $\gamma_{0}$ too is simple, and $\bar{\gamma}_{0}:=\left.\left(\gamma_{0}^{X_{0}}\right)^{-1}\right|_{\mathfrak{A}_{I}}$ is localised in $X_{0}$. We will show that $\bar{\gamma}_{0}$ is equivalent to $\bar{\gamma}$. As $\mathcal{X}$ is connected, it suffices to consider the case $X \cup X_{0} \subset Y$, for some $Y \in \mathcal{X}$. But then $\gamma^{Y}, \gamma_{0}^{Y} \in \operatorname{Aut} \mathcal{E}(Y)$, and any unitary $v \in I\left(\gamma, \gamma_{0}\right)$ yields a unitary $\left(\gamma^{Y}\right)^{-1}(v) \in I\left(\bar{\gamma}_{0}, \bar{\gamma}\right)$, as is verified by a straightforward computation. This completes the proof.

As explained above, the preceding results guarantee that we are in a situation where Proposition A.3 is applicable to each $\Delta(X)$. Thus in particular, an object $\rho \in \Delta(X)$ is finite-dimensional (i.e., possesses a conjugate) iff it has finite statistics. It is therefore natural to denote with $\Delta(X)_{\mathrm{f}}$ the full subcategory of these objects (and with $\Delta_{\mathrm{f}}$ the full subcategory of $\Delta$ consisting of all objects contained in some $\left.\Delta(X)_{\mathrm{f}}\right)$ and to summarise the situation as follows:

Proposition 3.17 The map $X \longmapsto\left(\Delta(X)_{\mathrm{f}}, \epsilon\right)$ is an isotonous net of (mutually equivalent) symmetric $r C^{*}$-categories with conjugates.

The importance of this structure lies in the fact that it is possible to construct from it (by a deep result of Doplicher and Roberts [2.9, 30]) a compact group $\mathcal{G}$ and an isotonous field net $\mathcal{F}(X), X \in \mathcal{X}$ with normal commutation relations whose gauge invariant part contains in a suitable sense representatives of all (irreducible finite-dimensional) elements of $\operatorname{DHR}\left(\mathcal{X}, \pi_{I}\right) / \cong$. (We do not want want to explain these notions here but merely mention that the construction of $\mathcal{G}$ and $\mathcal{F}(X)$ from $\left(\Delta(X)_{\mathrm{f}}, \epsilon\right)$ is performed separately for each $X$; 
the isotony of $X \longmapsto \mathcal{F}(X)$ then follows from that of $X \longmapsto \Delta(X)_{\mathrm{f}}$, and the property of all $\Delta(X)_{\mathrm{f}}$ being equivalent implies that $\mathcal{G}$ does not depend on $X$.) Physically, this result means that the field net describes all states containing a charge which is localisable in each $X \in \mathcal{X}$ in front of the chosen background, and that the values of that charge are described by the representation theory of $\mathcal{G}$. This is the situation usually formulated as a gauge field theory with global gauge group $\mathcal{G}$.

\subsection{The Action of a Symmetry Group}

We now discuss the algebraic consequences of the presence of a group of spacetime symmetries. While we will specialise later on to the case of the translation group and questions related to the spectrum of the energy-momentum operator, the following assumptions are sufficient for the time being:

g1 $G$ is a group which acts on the set $\mathcal{X}$ of localisation regions in such a way that each $g: \mathcal{X} \longrightarrow \mathcal{X}$ satisfies

$$
\begin{array}{lll}
g X_{1} \subset g X_{2} & \text { if } & X_{1} \subset X_{2} \\
g X_{1} \times g X_{2} & \text { if } & X_{1} \times X_{2}
\end{array}
$$

g2 The group $G$ acts by automorphisms $\alpha_{g}, g \in G$ on the $\mathrm{C}^{*}$-algebra $\mathcal{B}\left(\mathcal{H}_{I}\right)$, and the net $X \longmapsto \mathfrak{A}_{I}(X)$ is covariant under this action, i.e.,

$$
\mathfrak{A}_{I}(g X)=\alpha_{g}\left(\mathfrak{A}_{I}(X)\right), \quad g \in G, X \in \mathcal{X} .
$$

(Since all automorphisms of $\mathcal{B}\left(\mathcal{H}_{I}\right)$ are inner, there exists for each $g \in G$ a unitary $U_{I}(g)$ such that $\alpha_{g}=\operatorname{Ad} U_{I}(g)$; in general, $g \longmapsto U_{I}(g)$ is a group homomorphism only up to a phase.)

g3 There exists a $G$-covariant enlargement $\left(e_{n}\right)_{n \in \mathbb{N}}$, i.e., an enlargement that fulfils

$$
e_{n}(g X)=g e_{n}(X), \quad g \in G, X \in \mathcal{X}
$$

These assumptions permit to amend the structures established in the previous sections with natural symmetries under $G$, and the various nets of categories constructed in Prop. 3.9, 3.13 and 3.17 turn out to be covariant with respect to $G$.

To begin with, we notice that (as a consequence of $\mathbf{g 2}$ ) each $\alpha_{g}$ leaves the $\mathrm{C}^{*}$-subalgebra $\mathfrak{A}_{I}=\bar{\bigcup}_{X} \mathfrak{A}_{I}(X){ }^{\|\cdot\|} \subset \mathcal{B}\left(\mathcal{H}_{I}\right)$ invariant. Therefore the $\mathrm{W}^{*}$-category $\operatorname{Hom}\left(\mathfrak{A}_{I}, \mathcal{B}\left(\mathcal{H}_{I}\right)\right)$ carries an action of $G$ by autofunctors $\beta_{g}$ defined (on objects $\rho$ resp. on morphisms $t \in I(\sigma, \tau)$ ) by

$$
\beta_{g}(\rho):=\alpha_{g} \circ \rho \circ \alpha_{g}^{-1} \equiv \rho_{g} \quad \text { and } \quad \beta_{g}(t):=\left(\tau_{g}, \alpha_{g}(t), \sigma_{g}\right) .
$$

It is easily checked that this action indeed respects all structures involved (addition, composition, norm, *-operation and $\mathrm{w}^{*}$-topology). Moreover g1 and g2 (and duality) also imply $\mathfrak{A}_{I}\left((g X)^{\prime}\right)=\alpha_{g}\left(\mathfrak{A}_{I}\left(X^{\prime}\right)\right)$, which entails that an object $\rho$ is localised in $X$ iff $\rho_{g}$ is localised in $g X$. From this one concludes that $\rho$ is transportable iff $\rho_{g}$ is. (Notice that $\rho$ 
and $\rho_{g}$ need not be equivalent.) Therefore $\beta_{g}$ is an automorphism ( $\mathrm{C}^{*}$-autofunctor $)$ of $\Delta$ and maps the subcategory $\Delta(X)$ onto $\Delta(g X)$; thus, the net $X \longmapsto \Delta(X)$ of $\mathrm{W}^{*}$-categories is covariant under $G$.

In a next step, we want to convince ourselves that in restriction to $\Delta(X)$, each $\beta_{g}$ is a monoidal (iso-)functor, i.e., that the functor $\beta_{g}: \Delta(X) \longrightarrow \Delta(g X)$ is compatible with the monoidal products. The following lemma shows that this is the case, drawing upon the existence of a covariant enlargement $\left(e_{n}\right)_{n \in \mathbb{N}}$ demanded in $\mathbf{g} \mathbf{3}$ :

\section{Lemma 3.18}

i. The net $X \longmapsto \mathcal{E}(X)$ of $C^{*}$-algebras is covariant, $\alpha_{g}(\mathcal{E}(X))=\mathcal{E}(g X)$.

ii. The inclusions $\Delta(X) \hookrightarrow \operatorname{End} \mathcal{E}(X)$ are compatible with the symmetries $g \in G$, i.e., for any $\rho \in \Delta(X)$, the endomorphisms $\alpha_{g} \circ \rho^{X} \circ \alpha_{g}^{-1}$ and $\left(\rho_{g}\right)^{g X}$ (of $\mathcal{E}(g X)$ ) coincide.

iii. For any $X \in \mathcal{X}, g \in G$, one has

$$
\begin{aligned}
\beta_{g}\left(\rho_{1} \underset{X}{\rho_{2}}\right)=\beta_{g}\left(\rho_{1}\right) \underset{g X}{\stackrel{\beta}{\beta_{g}}\left(\rho_{2}\right)} & \text { if } \rho_{1}, \rho_{2} \text { are objects in } \Delta(X), \\
\beta_{g}\left(t_{1} \underset{X}{\otimes} t_{2}\right)=\beta_{g}\left(t_{1}\right) \underset{g X}{\otimes} \beta_{g}\left(t_{2}\right) & \text { if } t_{1}, t_{2} \text { are morphisms in } \Delta(X) .
\end{aligned}
$$

Proof: i. Since the net $\mathfrak{A}_{I}$ and the given enlargement $\left(e_{n}\right)_{n \in \mathbb{N}}$ are covariant, one has $\alpha_{g}\left(\mathfrak{A}_{I}\left(X^{(n)}\right)\right)=\mathfrak{A}_{I}\left((g X)^{(n)}\right)$. Taking the weak closures, then the union over all $n \in \mathbb{N}$ and finally the norm closures, one obtains from this the equality $\alpha_{g}(\mathcal{E}(X))=\mathcal{E}(g X)$.

ii. Let $\rho \in \Delta(X)$. We want to show that $\left(\rho_{g}\right)^{g X}$ and $\alpha_{g} \circ \rho^{X} \circ \alpha_{g}^{-1}$ coincide on $\mathfrak{A}_{I}\left(g X^{(n)}\right)^{\prime \prime}$ for all $n \in \mathbb{N}$. From Lemma 3.4 it follows that $\rho^{X}$ coincides on $\mathfrak{A}_{I}\left(X^{(n)}\right)^{\prime \prime}$ with $\operatorname{Ad} w$, where $w$ is any unitary which implements $\rho$ on $\mathfrak{A}_{I}\left(X^{(n)}\right)$. Therefore $\alpha_{g} \circ \rho^{X} \circ \alpha_{g}^{-1}$ coincides on $\alpha_{g}\left(\mathfrak{A}_{I}\left(X^{(n)}\right)\right)^{\prime \prime}$ with $\operatorname{Ad} \alpha_{g}(w)$, and thus, equivalently, with $\operatorname{Ad} \tilde{w}$, where $\tilde{w}$ is any unitary which implements $\alpha_{g} \circ \rho \circ \alpha_{g}^{-1}=\rho_{g}$ on $\alpha_{g}\left(\mathfrak{A}_{I}\left(X^{(n)}\right)\right)=\mathfrak{A}_{I}\left((g X)^{(n)}\right)$. But with such a $\tilde{w}$, Ad $\tilde{w}$ also coincides on $\mathfrak{A}_{I}\left((g X)^{(n)}\right)^{\prime \prime}$ with $\left(\rho_{g}\right)^{g X}$, by the definition of the latter. This proves the asserted equality.

iii. For given $g \in G$ and $X \in \mathcal{X}$, the isomorphism $\alpha_{g}: \mathcal{E}(X) \longrightarrow \mathcal{E}(g X)$ induces an isomorphism $\tilde{\beta}_{g}: \operatorname{End} \mathcal{E}(X) \longrightarrow \operatorname{End} \mathcal{E}(g X)$ (defined with formulae analogous to (3.1)), and Part ii implies that

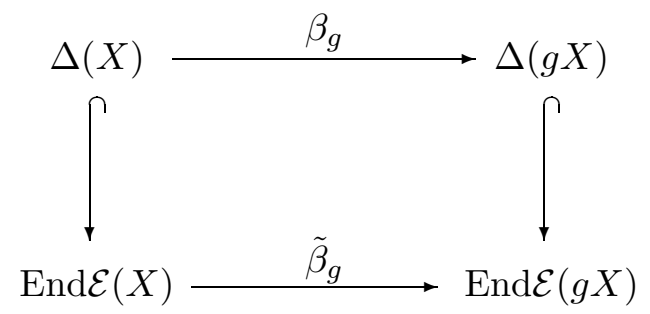

is a commutative diagram in the category of $\mathrm{C}^{*}$-categories. Since the inclusions as well as $\tilde{\beta}_{g}$ are monoidal functors, the functor $\beta_{g}$ is monoidal too, which was to be shown.

As to the next piece of structure, namely the symmetries $\epsilon^{X}$ on the monoidal categories $\Delta(X)$, we observe that these are preserved under the monoidal functors $\beta_{g}: \Delta(X) \longrightarrow$ $\Delta(g X)$ as well. Indeed, as the action of $G$ on $\mathcal{X}$ was assumed to respect the causal disjointness relation $X$, it also respects the relation $\perp$ introduced before Lemma 3.12. This fact allows one to invoke the uniqueness property in Prop. 3.13 in order to conclude 
that the symmetry $(\sigma, \tau) \longmapsto \beta_{g}^{-1}\left(\epsilon^{g X}\left(\sigma_{g}, \tau_{g}\right)\right)$ obtained on $\Delta(X)$ by pulling back the one from $\Delta(g X)$ via $\beta_{g}$, is identical to the symmetry $\epsilon^{X}$. Summarising, we thus obtain:

Proposition 3.19 The net $X \longmapsto\left(\Delta(X), \epsilon^{X}\right)$ of symmetric $r W^{*}$-categories is covariant under the action of $G$, i.e., each $\beta_{g}: \Delta(X) \longrightarrow \Delta(g X)$ is an isomorphism of symmetric $r W^{*}$-categories.

As an immediate consequence, $\beta_{g}$ restricts to an isofunctor $\beta_{g}: \Delta(X)_{\mathrm{f}} \longrightarrow \Delta(g X)_{\mathrm{f}}$ between the subcategories of finite-dimensional objects.

\subsection{Charge Transporting Cocycles}

In a net of $\mathrm{rW}^{*}$-categories covariant under the action of a group $G$, it is natural to introduce the notions of covariant objects and of charge transporting cocycles. Certain continuity properties are necessary, and we therefore make the following additional assumptions:

g4 $G$ is a finite-dimensional connected Lie group. Its action $\alpha$ on $\mathcal{B}\left(\mathcal{H}_{I}\right)$ is weakly continuous (i.e., each function $g \longmapsto \alpha_{g}(a), a \in \mathcal{B}\left(\mathcal{H}_{I}\right)$ is continuous in the weak operator topology).

g5 The action $g: X \longmapsto g X$ of $G$ on the set $\mathcal{X}$ has the property that for each $X \in \mathcal{X}$ there exists some neighbourhood $K_{X}$ of the unit $\mathbf{1} \in G$ such that the set of regions $\left\{Y \in \mathcal{X} \mid Y \supset g X, g \in K_{X}\right\}$ is nonempty and connected.

Remarks: 1 . The weak continuity of $\alpha$ implies for the action $\beta: G \longrightarrow$ Aut $\Delta$ on the category $\Delta$ that each function $g \longmapsto \beta_{g}(t), \quad t \in$ Mor $\Delta$ is continuous when Mor $\Delta$ is equipped with the pullback of the weak operator topology via the map Mor $\Delta \longrightarrow$ $\mathcal{B}\left(\mathcal{H}_{I}\right): t \longmapsto t$. (This was called the "overall w*-topology" in Section A.1.) In the sequel, continuity of Mor $\Delta$-valued functions (on $G$ ) will always be understood with respect to this topology.

2. The assumption $\mathbf{g} \mathbf{5}$ may also be viewed as a continuity property in the sense that the image of some $X \in \mathcal{X}$ under a "small" group element $g \in K_{X}$ still remains close to $X$, i.e., $g X \cup X \subset Y$. As main examples for such an action, one may think of any combination of one of the examples of sets $\mathcal{X}$ discussed in Section 3.2 (double cones, spacelike cones, opposite spacelike cones - arbitrary or upright in some given Lorentz system) with some subgroup $G \subset \mathcal{P}_{+}^{\uparrow}$ of the Poincaré group acting pointwise (i.e., $g X=\{g x \mid x \in X\}$ ) and leaving $\mathcal{X}$ invariant. As an aside, we notice that this indeed makes the enlargements discussed there $G$-covariant, so $\mathbf{g} 3$ holds. If $G$ contains translations only, then any compact (and convex, say) neighbourhood of 0 can be chosen for the neighbourhoods $K_{X}$ appearing in $\mathbf{g} 5$; a bit more care is required in the other cases.

Now let $\tilde{G}$ be the covering group of $G$. It acts in an obvious way on $\mathcal{B}\left(\mathcal{H}_{I}\right), \Delta$ and on $\mathcal{X}$, and without risk of confusion these actions of $\tilde{G}$ will be denoted with the same symbols as those of $G$. Notice that the properties assumed for $G$ in $\mathbf{g} \mathbf{4}$ and $\mathbf{g} \mathbf{5}$ carry over to $\tilde{G}$.

Definition: Let $\rho \in \Delta$. A charge transporting cocycle for $\rho$ is a continuous function $\Gamma: \tilde{G} \longrightarrow$ Mor $\Delta$ such that

- for each $g \in \tilde{G}, \Gamma(g)$ is a unitary morphism from $\rho_{g}$ to $\rho$; 
- for all $g, g^{\prime} \in \tilde{G}$, the cocycle equation $\Gamma(g) \circ \beta_{g}\left(\Gamma\left(g^{\prime}\right)\right)=\Gamma\left(g g^{\prime}\right)$ holds.

The set of all these cocycles will be denoted with $Z(\rho)$. The object $\rho$ is called covariant if $Z(\rho) \neq \emptyset$.

Remarks: 1. As the monoidal unit $\iota$ is invariant under $\beta$, it is trivially covariant, and the constant function $g \longmapsto \mathbf{1}_{\iota}$ is an element of $Z(\iota)$. Other objects need not be covariant, in general. Of course, covariance is a property invariant under unitary equivalence; each unitary $u: \rho \rightarrow \tilde{\rho}$ induces a bijection from $Z(\rho)$ to $Z(\tilde{\rho})$ via $\Gamma \longmapsto{ }^{u} \Gamma ;{ }^{u} \Gamma(g)=u \Gamma(g) \beta_{g}\left(u^{*}\right)$.

2. If $\alpha: G \longrightarrow \operatorname{Aut} \mathcal{B}\left(\mathcal{H}_{I}\right)$ is the adjoint action of a strongly continuous unitary group $U_{I}(g), g \in G$, then the above formulation of covariance coincides with the traditional one: The group $U_{I}$ induces (for each object $\rho$ ) a bijective correspondence (via the formula $\left.V(g)=\Gamma(g) U_{I}(g)\right)$ between $Z(\rho)$ and the set of strongly continuous unitary representations $V: \tilde{G} \longrightarrow \mathcal{B}\left(\mathcal{H}_{I}\right)$ which satisfy $\operatorname{Ad} V(g) \circ \rho=\rho \circ \alpha_{g}$.

3. In the definition of covariant objects, $\tilde{G}$ instead of $G$ has been used in order not to exclude fermionic objects from the outset. In the above-mentioned examples with $G \subset \mathcal{P}_{+}^{\uparrow}$, a difference between $G$ and $\tilde{G}$ arises when $G$ contains a subgroup of rotations. Moreover on a technical level, the property of $\tilde{G}$ being simply connected will be of central importance at two different points in the sequel (namely in the proofs of Lemmas 3.20 and 3.21).

We denote with $\Delta_{\mathrm{f}, \mathrm{c}}\left(\right.$ resp. $\left.\Delta(X)_{\mathrm{f}, \mathrm{c}}\right)$ the full subcategory of all covariant objects in $\Delta_{\mathrm{f}}$ (resp. $\Delta(X)_{\mathrm{f}}$ ). It will be shown that each $\Delta(X)_{\mathrm{f}, \mathrm{c}}$ is - like $\Delta(X)_{\mathrm{f}}-$ an $\mathrm{rW}^{*}$-category with conjugates. As far as the monoidal operations are concerned, it has to be checked that the covariant objects are closed under the product and under conjugates. Regarding the former, it seems straightforward to associate to each pair of cocycles $\Gamma_{j} \in Z\left(\rho_{j}\right)$, $\rho_{j} \in \Delta(X)_{\mathrm{f}, \mathrm{c}}, j=1,2$, a cocycle $\Gamma_{1} \otimes \Gamma_{2} \in Z\left(\rho_{1} \rho_{2}\right)$ via the formula

$$
\left(\Gamma_{1} \otimes \Gamma_{2}\right)(g)=\Gamma_{1}(g) \otimes \Gamma_{2}(g) .
$$

However, the right-hand side of this formula is only a valid expression if $\Gamma_{1}(g)$ and $\Gamma_{2}(g)$ are morphisms in some monoidal category $\Delta(Y)$. This need not be fulfilled for every $g \in \tilde{G}$, but according to assumption $\mathbf{g} 5$ it is the case in some neighbourhood of $\mathbf{1} \in \tilde{G}$.

Similarly, given a pair $(\rho, \bar{\rho})$ of conjugate objects in $\Delta(X)_{\mathrm{f}, \mathrm{c}}$ and a standard solution $(r, \bar{r}) \in \mathcal{R}(\rho, \bar{\rho})$ of the conjugate equations, it is natural to expect a bijection between $Z(\rho)$ and $Z(\bar{\rho})$ via the formula

$$
\Gamma \longmapsto \bar{\Gamma}, \quad \bar{\Gamma}(g)=(\Gamma(g))^{\dagger},
$$

where $\dagger: I\left(\rho_{g}, \rho\right) \longrightarrow I\left(\bar{\rho}_{g}, \bar{\rho}\right)$ denotes the conjugation induced by the solutions $(r, \bar{r})$ and $\left(\beta_{g}(r), \beta_{g}(\bar{r})\right)$ (cf. Appendix A.4 for a summary of these notions), i.e.,

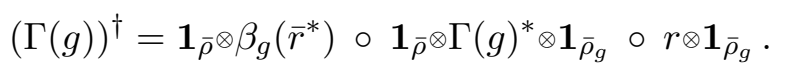

The same kind of restriction on $g \in \tilde{G}$ applies here. Nevertheless the above (well-known) formulae yield the desired result and are compatible, since one has for each region $X \in \mathcal{X}$ : 


\section{Lemma 3.20}

i. An associative binary operation on $\bigcup\left\{Z(\rho) \mid \rho \in \Delta(X)_{\mathrm{f}, \mathrm{c}}\right\}$ is given by the maps

$$
Z\left(\rho_{1}\right) \times Z\left(\rho_{2}\right) \longrightarrow Z\left(\rho_{1} \rho_{2}\right):\left(\Gamma_{1}, \Gamma_{2}\right) \longmapsto \Gamma_{1} \otimes \Gamma_{2},
$$

where $\Gamma_{1} \otimes \Gamma_{2}$ is uniquely defined by (3.2) (in some neighbourhood of $\mathbf{1} \in \tilde{G}$ ).

ii. If $\rho, \bar{\rho} \in \Delta(X)_{\mathrm{f}, \mathrm{c}}$ and if $(r, \bar{r}) \in \mathcal{R}(\rho, \bar{\rho})$ is standard, then one has an involution

$$
Z(\rho) \longrightarrow Z(\bar{\rho}): \quad \Gamma \longmapsto \bar{\Gamma},
$$

where $\bar{\Gamma}$ is uniquely defined by (3.3) (in some neighbourhood of $\mathbf{1} \in \tilde{G}$ ).

iii. Let $\left(r_{j}, \bar{r}_{j}\right) \in \mathcal{R}\left(\rho_{j}, \bar{\rho}_{j}\right), j=1,2$ be standard solutions and let $(r, \bar{r}) \in \mathcal{R}\left(\rho_{1} \rho_{2}, \bar{\rho}_{2} \bar{\rho}_{1}\right)$ be their product. If the conjugations $\dagger$ are taken with respect to these solutions, then one has for all $\Gamma_{j} \in Z\left(\rho_{j}\right)$ :

$$
\overline{\Gamma_{1} \otimes \Gamma_{2}}=\bar{\Gamma}_{2} \bar{\Gamma}_{1} .
$$

Proof: In Parts i and ii, the subtle point is to verify that the expressions for $\Gamma_{1} \otimes \Gamma_{2}$ and $\bar{\Gamma}$ yield well-defined elements of $Z\left(\rho_{1} \rho_{2}\right)$ (resp. $\left.Z(\bar{\rho})\right)$. To this end, let $K \subset \tilde{G}$ be a (connected) neighbourhood of $\mathbf{1} \in \tilde{G}$, and let $Y \in \mathcal{X}$ be such that $Y \supset K X$. For $g \in K, \Gamma_{1}(g) \otimes \Gamma_{2}(g)$ and $(\Gamma(g))^{\dagger}$ exist as morphisms in the monoidal category $\Delta(Y)$. As morphisms in $\Delta$, they are independent of $Y$ due to the connectedness assumption in $\mathbf{g} \mathbf{4}$ and Lemma 3.5. It is now immediately checked that the morphisms thus defined are unitary (in the case of $\Gamma(g)^{\dagger}$, this relies on the solution $(r, \bar{r})$ being standard, cf. Lemma A.5) and that the cocycle equation is satisfied for arguments $g$ and $g^{\prime}$ such that $\left\{g, g^{\prime}, g g^{\prime}\right\} \subset K$. To obtain the continuity properties (on $K$ ), first notice that for any continuous Mor $\Delta$-valued function $g \longmapsto t(g)$, the functions $g \longmapsto \mathbf{1}_{\tau} \otimes t(g)$ (for any fixed object $\tau \in \Delta(Y)$ ) and $g \longmapsto t(g) \otimes \mathbf{1}_{\sigma(g)}$ (for any (!) object-valued function $\left.g \longmapsto \sigma(g) \in \Delta(Y)\right)$ are continuous, cf. Lemma 3.8 and its proof. Second notice that if $g \longmapsto t_{1}(g)$ and $g \longmapsto t_{2}(g)$ are two continuous uniformly bounded functions (with matching sources and targets) one of which is unitary, then $g \longmapsto t_{1}(g) \circ t_{2}(g)$ is continuous. Combining all these facts, one obtains that $\left(\Gamma_{1} \otimes \Gamma_{2}\right)(g)=\mathbf{1}_{\rho_{1}} \otimes \Gamma_{2}(g) \circ \Gamma_{1}(g) \otimes \mathbf{1}_{\rho_{2, g}}$ is continuous. In the case of $\bar{\Gamma}$ one obtains similarly (from the continuity of $g \longmapsto \Gamma(g)^{*}$ ) that the product of the first two terms appearing in $(\Gamma(g))^{\dagger}$, namely $\mathbf{1}_{\bar{\rho} \otimes}\left(\beta_{g}\left(\bar{r}^{*}\right) \circ \Gamma(g)^{*} \otimes \mathbf{1}_{\bar{\rho}_{g}}\right)$ is continuous. But since composition (on the right) with $r \otimes \mathbf{1}_{\bar{\rho}_{g}}=\left(\bar{\rho} \rho \bar{\rho}_{g}, r, \bar{\rho}_{g}\right)$ preserves this property, $g \longmapsto \bar{\Gamma}(g)$ is continuous too. We have thus obtained the restrictions $\left.\left(\Gamma_{1} \otimes \Gamma_{2}\right)\right|_{K}$ and $\left.\bar{\Gamma}\right|_{K}$ of the desired cocycles. The homotopy argument presented in Appendix B (drawing on the cocycle equation and on $\tilde{G}$ being simply connected) can now be invoked in order to conclude that these restrictions actually define unique cocycles $\Gamma_{1} \otimes \Gamma_{2} \in Z\left(\rho_{1} \rho_{2}\right)$ and $\bar{\Gamma} \in Z(\bar{\rho})$. The remaining assertions, namely the associativity in Part i, the involution property in Part ii and the equality asserted in Part iii are readily verified for $g \in K$ (see Appendix A.4 for the algebraic arguments concerning the conjugation). By the homotopy argument they therefore hold for every $g \in \tilde{G}$.

Next, we consider the behaviour of the charge transporting cocycles under direct sums and subobjects. As to the former, the situation is easy: if $\rho=\bigoplus \rho_{j}$ is a finite direct sum (with isometries $w_{j}: \rho_{j} \hookrightarrow \rho$ ), of covariant objects $\rho_{j}, j=1, \ldots, J$, then $\rho$ is covariant too: a cocycle $\Gamma \in Z(\rho)$ can be obtained from cocycles $\Gamma_{j} \in Z\left(\rho_{j}\right)$ by setting

$$
\Gamma(g):=\sum_{j} w_{j} \Gamma_{j}(g) \beta_{g}\left(w_{j}^{*}\right)
$$


(The algebraic properties are obvious, and the continuity follows from an argument like in the proof of Lemma 3.20.) This cocycle, called the direct sum of the family $\left(\Gamma_{j}\right)_{j=1, \ldots, J}$ (via the isometries $w_{j}$ ), satisfies $E_{j} \Gamma(g)=\Gamma(g) \beta_{g}\left(E_{j}\right)$ for the projections $E_{j}:=w_{j} w_{j}^{*}$, but an analogous identity will not be true for arbitrary projections $E \in I(\rho, \rho)$. Similarly, given an isometry $w: \sigma \hookrightarrow \rho$ identifying $\sigma$ as a subobject of $\rho$, one might be tempted to define for $\Gamma \in Z(\rho)$ a projected cocycle,

$$
w^{*} \Gamma(g)=w^{*} \Gamma(g) \beta_{g}(w),
$$

but $w^{*} \Gamma \in Z(\sigma)$ if and only if the final projection $E:=w w^{*}$ fulfils

$$
E \Gamma(g)=\Gamma(g) \beta_{g}(E) ;
$$

in this case we will say that the isometry $w$ projects the cocycle $\Gamma$. Notice in particular that it is not immediately obvious whether covariance is inherited by subobjects.

The above-mentioned difficulties motivate to introduce the notion of natural cocycles: Definition: Let $\rho$ be an object of $\Delta_{\mathrm{f}, \mathrm{c}}$. An element of

$$
Z_{0}(\rho):=\left\{\Gamma \in Z(\rho) \mid \Gamma(g) \beta_{g}(t) \Gamma(g)^{*}=t \quad \text { for all } g \in \tilde{G}, t \in I(\rho, \rho)\right\}
$$

is called a natural charge transporting cocycle.

For irreducible objects, all charge transporting cocycles are natural, but for reducible ones, the natural cocycles have better properties than general ones in several (related) respects:

- It has already been noticed above that each unitary $u: \rho \rightarrow \tilde{\rho}$ induces a map $Z(\rho) \longrightarrow Z(\tilde{\rho}): \Gamma \longmapsto{ }^{u} \Gamma$. If (and only if) $\Gamma \in Z_{0}(\rho)$, then ${ }^{u} \Gamma \in Z_{0}(\tilde{\rho})$ is independent of the unitary $u$, so the bijection between the subsets of natural cocycles belonging to equivalent objects is canonical.

- If $\sigma$ is a subobject of $\rho$, then all isometries $w: \sigma \hookrightarrow \rho$ project a given $\Gamma \in Z_{0}(\rho)$ on the same element ${ }^{w^{*}} \Gamma$ of $Z_{0}(\sigma)$. Thus there exists a canonical map from $Z_{0}(\rho)$ to $Z_{0}(\sigma)$. This map is onto, as will follow from Lemma 3.21.

In order to conclude that the subcategory $\Delta_{\mathrm{f}, \mathrm{c}} \subset \Delta_{\mathrm{f}}$ is closed under subobjects, it must be guaranteed that natural cocycles exist for all covariant objects $\rho$ of $\Delta_{\mathrm{f}}$. This is done in Part $\mathrm{i}$ of the next lemma, which is based on the fact that the $\mathrm{C}^{*}$-algebras $I(\rho, \rho)$ are finite-dimensional and — once again — on $\tilde{G}$ being simply connected. Other structural properties of the sets of natural cocycles are put on record as well:

Lemma 3.21 Let $\rho$ be an object of $\Delta_{\mathrm{f}, \mathrm{c}}$. Then:

i. $Z_{0}(\rho) \neq \emptyset$.

ii. $Z_{0}(\rho)$ is canonically isomorphic to the cartesian product $\times_{j} Z_{0}\left(\rho_{j}\right)$, where $\rho_{j}(j=$ $1, \ldots, J)$ are pairwise disjoint (!) objects of $\Delta$ such that $\rho \cong \bigoplus_{j} \rho_{j}$.

iii. For any number $N \in \mathbb{N}$, the sets $Z_{0}(N \rho)$ and $Z_{0}(\rho)$ are canonically isomorphic.

Proof: i. Let $\rho$ be covariant and $\Gamma \in Z(\rho)$. Then $\psi_{g}(t):=\Gamma(g) \beta_{g}(t) \Gamma(g)^{*}$ defines an action of $\tilde{G}$ by automorphisms of the $\mathrm{C}^{*}$-algebra $I(\rho, \rho)$. Since both $I(\rho, \rho)$ and $\tilde{G}$ are finite-dimensional, the weak continuity of each $g \longmapsto \beta_{g}(t), \quad t \in I(\rho, \rho)$ implies that $g \longmapsto \psi_{g}$ is uniformly continuous. As such it is of the form $\psi_{g}(t)=B(g) t B(g)^{*}$ with 
a uniformly continuous representation $g \longmapsto B(g) \in I(\rho, \rho)$, cf. [32], Ch. 2.6. Setting $\Gamma_{0}(g):=B(g)^{*} \Gamma(g)$, it is then readily verified that $\Gamma_{0}$ is an element of $Z_{0}(\rho)$.

ii. Let $\rho$ and the family $\rho_{j}$ be as in the assertion and choose a family $w_{j}: \rho_{j} \hookrightarrow \rho$ of pairwise orthogonal isometries performing the direct sum decomposition. Then a map from $Z_{0}(\rho)$ to $X_{j} Z_{0}\left(\rho_{j}\right)$ is given by $\Gamma \longmapsto\left({ }^{*}{ }^{*} \Gamma\right)_{j=1, \ldots, J}$. Due to the mutual disjointness of the $\rho_{j}$, it is easily seen that its inverse is the map which sends each family $\Gamma_{j} \in Z_{0}\left(\rho_{j}\right)$ on its direct sum (via the isometries $w_{j}$ ) and that both maps actually do not depend on the chosen family of isometries.

iii. The canonical map from $Z_{0}(N \rho)$ to $Z_{0}(\rho)$ is the projection $\Gamma \longmapsto{ }^{*} \Gamma$ via any isometry $w: \rho \hookrightarrow N \rho$, and its inverse is the direct sum of $N$ copies of one element of $Z_{0}(\rho)$ via any family of $N$ pairwise orthogonal isometries $w_{j}: \rho \hookrightarrow N \rho$. It is straightforward to verify that these maps have the asserted properties and that they are independent of the chosen isometries.

In view of Lemma 3.21 and the preceding discussion, it might seem advantageous to consider natural cocycles only and to define the covariance of $\rho$ by the condition $Z_{0}(\rho) \neq \emptyset$. With such a definition, it would however be far from obvious why the set of covariant objects is closed under the monoidal product. This is due to the difficulty that the product of natural cocycles need not be natural any more, cf. the example below. Nevertheless, the next lemma shows that at least as far as the conjugation is concerned, the natural cocycles are well-behaved.

Lemma 3.22 Let $\rho, \bar{\rho} \in \Delta(X)_{\mathrm{f}, \mathrm{c}}$ be conjugate to each other.

$i$. The conjugation $\Gamma \longmapsto \bar{\Gamma}$ maps $Z_{0}(\rho)$ onto $Z_{0}(\bar{\rho})$ and is independent of the standard solution $(r, \bar{r}) \in \mathcal{R}(\rho, \bar{\rho})$ used for its definition.

ii. If $\Gamma \in Z_{0}(\rho)$, then every isometry $w: \iota \hookrightarrow \bar{\rho} \rho$ projects the cocycle $\bar{\Gamma} \otimes \Gamma$ onto the trivial element of $Z_{0}(\iota)$.

Proof: Like in the proof of Lemma 3.20 we may restrict the attention to $g \in K$, where $K$ is a sufficiently small neighbourhood of $\mathbf{1} \in \tilde{G}$. Let $(r, \bar{r}) \in \mathcal{R}(\rho, \bar{\rho})$ be a standard solution and denote with $\dagger$ all conjugations induced by $(r, \bar{r})$ and $\left(\beta_{g}(r), \beta_{g}(\bar{r})\right)$. Then one has $\beta_{g}\left(t^{\dagger}\right)=\beta_{g}(t)^{\dagger}$ for any $t \in I(\bar{\rho}, \bar{\rho})$. Since $\bar{\Gamma}(g)=\Gamma(g)^{\dagger}$, the rules listed in Section A.4 yield $\left(\Gamma(g) \beta_{g}\left(t^{\dagger}\right) \Gamma(g)^{*}\right)^{\dagger}=\bar{\Gamma}(g) \beta_{g}(t) \bar{\Gamma}(g)^{*}$. Thus if $\Gamma$ is natural, then the left-hand side simplifies to $t$, so $\bar{\Gamma}$ is natural too. The independence of $\bar{\Gamma}$ of the chosen standard solution is seen as follows: According to Lemma A.6, any other standard solution has the form $u \star(r, \bar{r})$ with some unitary $u \in I(\rho, \rho)$, and the conjugation $\dagger^{\prime}$ induced by it (and its image under $\beta_{g}$ ) yields $\Gamma(g)^{\dagger^{\prime}}=u^{* \dagger} \Gamma(g)^{\dagger} \beta_{g}\left(u^{* \dagger}\right)^{-1}=u^{\dagger *} \bar{\Gamma}(g) \beta_{g}\left(u^{\dagger}\right)=u^{\dagger *} u^{\dagger} \bar{\Gamma}(g)=\bar{\Gamma}(g)$. This proves Part i.

ii. Let $w: \iota \hookrightarrow \bar{\rho} \rho$ be given and set $t:=\bar{r}^{*} \otimes \mathbf{1}_{\rho} \circ \mathbf{1}_{\rho} \otimes w \in I(\rho, \rho)$. With a computation analogous to (C.3), one sees that $\mathbf{1}_{\bar{\rho} \otimes} \beta_{g}(t) \Gamma(g)^{*} \circ r=\bar{\Gamma}(g) \otimes \mathbf{1}_{\rho_{g}} \circ \beta_{g}\left(\mathbf{1}_{\bar{\rho} \otimes t} \circ r\right)$. Since $\Gamma$ is natural, composing with $\mathbf{1}_{\bar{\rho} \otimes \Gamma}(g)$ on the left yields $\mathbf{1}_{\bar{\rho} \otimes t} \circ r=\bar{\Gamma}(g) \otimes \Gamma(g) \circ \beta_{g}\left(\mathbf{1}_{\bar{\rho} \otimes t} \circ r\right)$. But this implies $\mathbf{1}_{\iota}=w^{*} \circ(\bar{\Gamma} \otimes \Gamma)(g) \circ \beta_{g}(w)$ because $\mathbf{1}_{\bar{\rho} \otimes t} \circ r=w$ is an isometry.

Before giving the example which shows that the class of natural cocycles is not closed under the product, we briefly mention the well-known description (for each covariant object $\rho$ ) of the whole set $Z(\rho)$ if one natural cocycle $\Gamma_{0} \in Z_{0}(\rho)$ is fixed. In this lemma, if $A$ is a (finite-dimensional) $\mathrm{C}^{*}$-algebra, $\operatorname{Rep}(\tilde{G}, A)$ denotes the set of all continuous homomorphisms from $\tilde{G}$ to the group of unitaries in $A$, and $\mathfrak{Z} A$ denotes the centre of $A$. 
Lemma 3.23 Let $\rho \in \Delta_{\mathrm{f}, \mathrm{c}}$ and let $\Gamma_{0} \in Z_{0}(\rho)$ be given. Then the map

$$
\Gamma \longmapsto B: \quad B(g)=\Gamma(g) \Gamma_{0}(g)^{*}
$$

establishes a bijection between $Z(\rho)$ and $\operatorname{Rep}(\tilde{G}, I(\rho, \rho))$. The subset $Z_{0}(\rho)$ of natural cocycles corresponds to $\operatorname{Rep}(\tilde{G}, \mathfrak{Z} I(\rho, \rho))$.

(We omit a detailed proof and merely stress that since $g \longmapsto \Gamma_{0}(g)$ is continuous and unitary, the continuity of $g \longmapsto \Gamma(g)$ and that of $g \longmapsto B(g)$ are equivalent. Also, since $\Gamma_{0}$ is natural and satisfies the cocycle equation, the group law of $B$ is equivalent to the cocyle equation for $\Gamma$.)

Example: We will construct a cocycle $\Gamma \in Z_{0}(\rho)$ such that $\bar{\Gamma} \otimes \Gamma \notin Z_{0}(\bar{\rho} \rho)$. For definiteness, we choose the group $G=\tilde{G}=\mathbb{R}^{1+s}$ here, but any other group having nontrivial one-dimensional continuous unitary representations would be suited as well. Assume that there exists a cocycle $\Gamma_{0} \in Z_{0}(\rho)$ such that $\bar{\Gamma}_{0} \otimes \Gamma_{0} \in Z_{0}(\bar{\rho} \rho)$. Then any other cocycle $\Gamma \in Z_{0}(\rho)$ has the form $\Gamma(x)=B(x) \Gamma_{0}(x)$ with $B \in \operatorname{Rep}\left(\mathbb{R}^{1+s}, \mathfrak{Z} I(\rho, \rho)\right)$. Hence $(\bar{\Gamma} \otimes \Gamma)(x)=B(x)^{\dagger} \otimes B(x) \circ\left(\bar{\Gamma}_{0} \otimes \Gamma_{0}\right)(x)$, and $\bar{\Gamma} \otimes \Gamma$ is natural iff each $B(x)^{\dagger} \otimes B(x)$ is in the centre of $I(\bar{\rho} \rho, \bar{\rho} \rho)$.

Now let $w_{j}: \sigma_{j} \hookrightarrow \rho$ (resp. $\bar{w}_{j}: \bar{\sigma}_{j} \hookrightarrow \bar{\rho}$ ) be a family of pairwise orthogonal isometries which performs a decomposition $\rho=\bigoplus_{j} \sigma_{j}$ (resp. $\bar{\rho}=\bigoplus_{j} \bar{\sigma}_{j}$ ) into mutually disjoint primary subobjects. Then the centre of $I(\rho, \rho)$ (resp. $I(\bar{\rho}, \bar{\rho}))$ is spanned by the projections $E_{j}:=w_{j} w_{j}^{*}\left(\right.$ resp. $\bar{E}_{j}:=\bar{w}_{j} \bar{w}_{j}^{*}$ ), and the general form for $B$ reads $B(x)=\sum_{j} e^{i p_{j} x} E_{j}$ with constant vectors $p_{j} \in \mathbb{R}^{1+s}$. Assuming - as already suggested by the notation - that $\sigma_{j}$ and $\bar{\sigma}_{j}$ are conjugate for each $j$, this implies $B(x)^{\dagger}=\sum_{j} e^{-i p_{j} x} \bar{E}_{j}$, and therefore $B(x)^{\dagger} \otimes B(x)=\sum_{k, l} e^{-i\left(p_{k}-p_{l}\right) x} \bar{E}_{k} \otimes E_{l}$, and we want to see why this last expression need not be an element of the centre of $I(\bar{\rho} \rho, \bar{\rho} \rho)$.

Assume that there exists an object $\tau$ which is a subobject of both $\bar{\sigma}_{i} \sigma_{j}$ and $\bar{\sigma}_{k} \sigma_{l}$ for some indices $i, j, k, l$ satisfying $i \neq k$ or $j \neq l$. Then there is some nonzero morphism $a: \bar{\sigma}_{k} \sigma_{l} \rightarrow \bar{\sigma}_{i} \sigma_{j}$. Setting $q:=\bar{w}_{i} \otimes w_{j} \circ a \circ \bar{w}_{k}^{*} \otimes w_{l}^{*}$, one verifies that

$$
B(x)^{\dagger} \otimes B(x) \circ q=e^{-i\left(p_{i}-p_{j}\right) x} q, \quad q \circ B(x)^{\dagger} \otimes B(x)=e^{-i\left(p_{k}-p_{l}\right) x} q .
$$

Thus if $i \neq j$ or $k \neq l$, then $B(x)^{\dagger} \otimes B(x)$ is not in the centre of $I(\bar{\rho} \rho, \bar{\rho} \rho)$ for generic values of the vectors $p_{j}$.

The above example makes use of the property of $\rho$ being reducible, but even in the case when $\rho \in \Delta_{\mathrm{f}, \mathrm{c}}$ is irreducible (and thus every $\Gamma \in Z(\rho)$ is natural), there seems to be no general argument showing that $\bar{\Gamma} \otimes \Gamma \in Z(\bar{\rho} \rho)$ is natural. (The difficulty which arises is that $\bar{\rho} \rho$ may contain some irreducible object $\sigma$ with multiplicity $N>1$; in this case an isometry $w_{N}: N \sigma \hookrightarrow \bar{\rho} \rho$ of course projects $\bar{\Gamma} \otimes \Gamma$, but there might exist isometries $w_{1}: \sigma \hookrightarrow \bar{\rho} \rho$ which do not. To cite two concrete cases, recall that if the gauge group is $\mathrm{SU}(2)$, then every product of two irreducible objects is free of multiplicities, whereas in the case of $\mathrm{SU}(3)$, one has $\rho_{8} \oplus \rho_{8} \subset \rho_{8} \rho_{8}$, where $\rho_{8}$ denotes some (irreducible, selfconjugate) object corresponding to the representation $\underline{8}$.) In view of this situation, it is legitimate to ask whether there exists a subclass of natural cocycles which is closed under the product. This suggests the following definition, closely related to what is called a "coherently covariant family of [objects]" in [30, 33]. 
Definition: A natural family of cocycles is a family $\Gamma_{\rho} \in Z_{0}(\rho), \rho \in \Delta_{\mathrm{f}, \mathrm{c}}$ of cocycles such that one has for all $g \in \tilde{G}$, all $\sigma, \tau \in \Delta_{\mathrm{f}, \mathrm{c}}$ and all $t: \sigma \rightarrow \tau$ :

$$
\Gamma_{\tau}(g) \beta_{g}(t)=t \Gamma_{\sigma}(g)
$$

A natural family $\left(\Gamma_{\rho}\right)_{\rho \in \Delta_{\mathrm{f}, \mathrm{c}}}$ is called monoidal if $\Gamma_{\rho_{1}} \otimes \Gamma_{\rho_{2}}=\Gamma_{\rho_{1} \rho_{2}}$ for all $\rho_{1}, \rho_{2} \in \Delta_{\mathrm{f}, \mathrm{c}}$.

Remarks: 1. Due to the behaviour of natural cocycles with respect to direct sums (cf. Lemma 3.21 and the discussion preceding it), a natural family of cocycles is determined by the choice of a cocycle for one representative of each equivalence class of irreducible objects.

2. If the natural family $\left(\Gamma_{\rho}\right)_{\rho \in \Delta_{\mathrm{f}, \mathrm{c}}}$ is monoidal, then it necessarily fulfils $\Gamma_{\iota}=\mathbf{1}_{\iota}$ and $\overline{\Gamma_{\rho}}=\Gamma_{\bar{\rho}}$. (The second identity follows for irreducible objects from Lemma 3.22 and carries over to reducible ones by naturality.)

With these notions, one may thus ask whether there exists a monoidal natural family of cocycles and how such families are classified. The answer to these questions depends of course on both the symmetry group $\tilde{G}$ and the gauge group $\mathcal{G}$, the latter appearing in the guise of the monoidal structure of $\Delta_{\mathrm{f}, \mathrm{c}}$. We will not discuss these issues further, but just illustrate them in two specific cases:

The most prominent (in physics) of the trivial examples is that where $G=\mathcal{P}_{+}^{\uparrow}$ is the Poincaré group. Since $\tilde{G}$ has no nontrivial finite-dimensional continuous unitary representations, the set $Z(\rho)$ of cocycles has exactly one element $\Gamma_{\rho}$ if $\rho$ is covariant. Therefore the unique natural family is a fortiori monoidal.

The situation is far less trivial if $G=\mathbb{R}^{1+s}$ is the spacetime translation group. Under physically reasonable assumptions concerning the action $\beta$ of $\tilde{G}$ (and using the net structure of $\Delta$ ), one can show the existence of a monoidal natural family of cocycles (cf. Chapter 4). One such family is completely characterised by a very different property (viz. the minimality of the energy-momentum spectrum), but depending on the gauge group, there may in general exist other monoidal natural families besides this minimal one. (For instance, if the gauge group is abelian, then each $\Gamma_{\rho}, \rho$ irreducible, can be multiplied by a phase function $x \longmapsto e^{i Q_{\rho} x}$, the vectors $Q_{\rho} \in \mathbb{R}^{1+s}$ being chosen suitably. Notice that the situation is not drastically different when $G=\mathbb{R} \oplus\left(\mathbb{R}^{s} \rtimes \mathrm{SO}(s)\right) \subset \mathcal{P}_{+}^{\uparrow}$ is the subgroup of $\mathcal{P}_{+}^{\uparrow}$ leaving a particular Lorentz system fixed; the possible shift $Q_{\rho} \in \mathbb{R}^{1+s}$ of the zero point of energy-momentum is replaced by a shift $Q_{\rho} \in \mathbb{R}$ of the energy only.)

The present section would not be complete without an explanation for choosing the terms "natural cocycle" and "natural family" in the above definitions. The reason is that for fixed $g \in \tilde{G}$, the property

$$
\Gamma_{\tau}(g) \beta_{g}(t)=t \Gamma_{\sigma}(g) \quad \text { for all } t: \sigma \rightarrow \tau
$$

means that the family $\Gamma_{\bullet}(g):=\left(\Gamma_{\rho}(g)\right)_{\rho \in \Delta_{\mathrm{f}, \mathrm{c}}}$ of unitaries is precisely what is known as a unitary natural transformation (or as a natural isomorphism)

$$
\Gamma_{\bullet}(g): \beta_{g} \Rightarrow \operatorname{Id}_{\Delta_{\mathrm{f}, \mathrm{c}}}
$$

from the autofunctor $\beta_{g}: \Delta_{\mathrm{f}, \mathrm{c}} \longrightarrow \Delta_{\mathrm{f}, \mathrm{c}}$ to the identity functor on the $\mathrm{rW}^{*}$-category $\Delta_{\mathrm{f}, \mathrm{c}}$. (See page 74 in Section A.1 for a brief sketch of these terms.) If moreover the natural 
family $\left(\Gamma_{\rho}\right)_{\rho \in \Delta_{\mathrm{f}, \mathrm{c}}}$ is monoidal, then $\Gamma_{\bullet}(g)$ is a monoidal natural transformation . The above reformulations do not depend on the validity of the cocycle equation (let alone on the continuity of the cocycles), but we note as an aside that each $\Gamma_{\rho}$ fulfilling the cocycle equation is equivalent to the following relation between natural transformations (for all $\left.g_{1}, g_{2} \in \tilde{G}\right)$ :

$$
\Gamma_{\bullet}\left(g_{1}\right) \otimes \Gamma_{\bullet}\left(g_{2}\right)=\Gamma_{\bullet}\left(g_{1} g_{2}\right) .
$$

Thus $g \longmapsto \Gamma_{\bullet}(g)$ is a group homomorphism from $\tilde{G}$ to the group (equipped with the "horizontal multiplication" $\bowtie)$ of unitary monoidal transformations between autofunctors of $\Delta_{\mathrm{f}, \mathrm{c}}$.

\footnotetext{
${ }^{4}$ Some caution is necessary here since $\Delta_{\mathrm{f}, \mathrm{c}}$ is not a monoidal category. But if $X, Y \in \mathcal{X}$ and $g \in \tilde{G}$ satisfy $X \cup g X \subset Y$ then $\beta_{g}$ and $\operatorname{Id}_{\Delta_{\mathrm{f}, \mathrm{c}}}$ restricted to $\Delta(X)_{\mathrm{f}, \mathrm{c}}$ and composed with the inclusion into $\Delta(Y)_{\mathrm{f}, \mathrm{c}}$ are monoidal functors between a pair of monoidal categories, and between such functors the notion of monoidal natural transformations is meaningful.
} 



\section{Chapter 4}

\section{Spectral Properties}

In the algebraic description of covariant objects and of charge transporting cocycles the actual form of the symmetry group (and to a certain extent that of the underlying spacetime) played no direct role. We now want to consider translation covariant charges and investigate the properties of their energy-momentum spectra.

The framework of the present chapter is basically the same as that of Chapter 3, but we consider the following special case:

- The background representation $\pi_{I}: \mathfrak{A}_{0} \longrightarrow \mathcal{B}\left(\mathcal{H}_{I}\right)$ is an infravacuum representation which fulfils the assumptions b1 (property B) and b2 (duality) for the set $\mathcal{X}$.

- The symmetry group $G$ is the group $\mathbb{R}^{1+s}$ of translations of Minkowski space, acting on $\mathcal{B}\left(\mathcal{H}_{I}\right)$ with the adjoint action $\alpha_{x}=\operatorname{Ad} U_{I}(x)$ of the minimal representation $U_{I}$ for $\left(\mathcal{H}_{I}, \pi_{I}\right)$. ( $E_{I}$ will denote the spectral family of $U_{I}$.)

As to the set $\mathcal{X}$ of localisation regions, we will restrict the attention to the two most important cases, namely either the set of all double cones in $\mathbb{R}^{1+s}$ (with $s \geq 2$ ) or the set of all spacelike cones $\mathbb{R}^{1+s}$ (with $s \geq 3$ ). (A further restriction to the subset of upright elements in some Lorentz system would be possible too.) The second case is naturally the one of main interest, but it is useful to recall the situation for pointlike charges first.

In both situations, all other assumptions of Chapter 3 are fulfilled, and we have thus the net $X \longmapsto \Delta(X)_{\mathrm{f}, \mathrm{c}}$ of symmetric $\mathrm{rW}^{*}$-categories with conjugates and for each object $\rho \in \Delta_{\mathrm{f}, \mathrm{c}}$ the sets $Z_{0}(\rho) \subset Z(\rho)$ of natural and general charge transporting cocycles.

\subsection{Cocycles and Their Spectra}

With the unitary group $U_{I}$ being fixed, one has for each object $\rho \in \Delta_{\mathrm{f}, \mathrm{c}}$ a unique identification between cocycles $\Gamma \in Z(\rho)$ and strongly continuous unitary representations

$$
x \longmapsto V_{\Gamma}(x):=\Gamma(x) U_{I}(x)
$$

which implement the action of $\alpha$ of $\mathbb{R}^{1+s}$ in the representation $\rho: \mathfrak{A}_{I} \longrightarrow \mathcal{B}\left(\mathcal{H}_{I}\right)$ (cf. the remarks in Section 3.7). The spectrum of $V_{\Gamma}$ is thus a set associated to $\Gamma$, and we write

$$
s(\Gamma):=\operatorname{sp} V_{\Gamma}=\operatorname{sp}\left(\Gamma(\cdot) U_{I}(\cdot)\right) \subset \mathbb{R}^{1+s} .
$$

\footnotetext{
${ }^{1}$ For simplicity we will frequently omit in this chapter the notational distinction between the morphisms $\Gamma(x)$ and the intertwining operators $\Gamma(x)$.
} 
Definition: The set $s(\Gamma)$ is called the spectrum of $\Gamma$. If $s(\Gamma) \subset \overline{\mathbf{V}}_{+}$for some $\Gamma \in Z(\rho)$, then the object $\rho \in \Delta_{\mathrm{f}, \mathrm{c}}$ is said to have positive energy. By the theorem of Borchers and Buchholz cited in the Introduction, one has in this case a unique minimal representation $U_{\rho \pi_{I}}: \mathbb{R}^{1+s} \longrightarrow \mathcal{B}\left(\mathcal{H}_{I}\right)$ for $\left(\mathcal{H}_{I}, \rho \pi_{I}\right)$, and it is natural to refer to the charge transporting cocycle corresponding to it (via $\Gamma_{\rho}(x)=U_{\rho \pi_{I}}(x) U_{I}(x)^{*}$ ) as the minimal cocycle for $\rho$. We will from now on reserve for it the notation $\Gamma_{\rho}$. Moreover, we put in this case $s(\rho):=s\left(\Gamma_{\rho}\right)$ and call $s(\rho)$ the spectrum of $\rho$. (Notice that $\Gamma_{\iota}=\mathbf{1}_{\iota}$.)

We list (without proof) some immediate properties of the map $\Gamma \longmapsto s(\Gamma)$. (Those parts which connect cocycles associated to different objects are trivial in the sense that they refer to the additive structure of $\Delta_{\mathrm{f}, \mathrm{c}}$ or to unitarily invariant concepts.)

\section{Lemma 4.1}

i. $s\left(\Gamma_{\iota}\right)=\operatorname{sp} U_{I}$.

ii. $s\left({ }^{u} \Gamma\right)=s(\Gamma)$ if $u$ is a unitary.

iii. $s(\Gamma)=\bigcup_{j} s\left(\Gamma_{j}\right)$ if $\Gamma$ is the direct sum of the family $\left(\Gamma_{j}\right)$.

iv. $s\left({ }^{w^{*}} \Gamma\right) \subset s(\Gamma)$ if $w$ is an isometry which projects $\Gamma$.

v. $s(\tilde{\Gamma})=q+s(\Gamma) \quad$ if $\tilde{\Gamma}(x)=e^{i q x} \Gamma(x), q \in \mathbb{R}^{1+s}$.

It follows in particular that the set of objects with positive energy is closed under subobjects and finite direct sums and that the corresponding family $\left(\Gamma_{\rho}\right)$ of minimal cocycles is natural.

In contrast, the nontrivial properties of the map $\Gamma \longmapsto s(\Gamma)$ are those connected with the monoidal structure of $\Delta_{\mathrm{f}, \mathrm{c}}$. They arise as a consequence of the local structure of the net $\mathfrak{A}_{I}$ and specific properties of the background $\pi_{I}$.

In the simplest case, namely when the background is the vacuum, then the following facts are well known [8,, 9$]$ :

\section{Proposition 4.2}

$i$. Each object $\rho \in \Delta_{\mathrm{f}, \mathrm{c}}$ has positive energy.

ii. $s(\rho)=s(\bar{\rho})$.

iii. $s\left(\rho_{1}\right)+s\left(\rho_{2}\right) \subset s(\sigma)$ if $\sigma$ is a subobject of $\rho_{1} \rho_{2}$.

These properties are in accordance with the idea that charged states are local excitations of the vacuum. As pointed out by Haag, iii for instance embodies the fact that if $\omega_{j}$ $(j=1,2)$ are states with charges $\left[\rho_{j}\right]$ and energy-momenta $p_{j}$ then there is also a state $\omega_{12}$ with the combined charge and with energy-momentum $p_{1}+p_{2}$; it may correspond to a situation which looks in one space-time region like $\omega_{1}$ and in a region very far apart (such that the interaction is negligible), like $\omega_{2}$. Other energy-momentum states (e.g. bound states) may of course exist in the combined sector due to an interaction; equality in iii is thus not to be expected in general. Property ii, on the other hand, is a manifestation of PCT symmetry; if the charges are carried by particles, it implies in particular that a particle and its antiparticle have equal masses.

We will briefly recall the derivation of these results in Section 4.3 . Here it should only be noted that it uses the translation invariance of the vacuum vector at technically crucial points. On the other hand, it is insensitive to whether the localisation regions are double cones or spacelike cones. (It would not carry over to the case of opposite spacelike cones, however.)

It is an important question whether the properties listed in Prop. 4.2 remain valid for the superselection theory in front of a true infravacuum. The picture that a background 
does not affect the dynamics of the charges in an essential way suggests that this should indeed be true.

We will see in the sequel that the answer to this question depends heavily on the localisation properties of the charges. In the case of compact localisation it will turn out to be positive and rather straightforward, whereas for charges localised in spacelike cones only a partial answer can be given.

For the discussion of compactly localised charges, it is useful to describe the spectrum $s(\Gamma)$ of a cocycle directly in terms of the operator-valued functions

$$
x \longmapsto \Gamma(x) E_{I}(D), \quad D \subset \mathbb{R}^{1+s}, \quad D \text { compact } .
$$

As a preparation, we recall that the spectrum $\operatorname{sp} A$ of a uniformly bounded, strongly continuous operator-valued function $A: \mathbb{R}^{1+s} \longrightarrow \mathcal{B}\left(\mathcal{H}_{I}\right)$ is, by definition, the support of its Fourier transform in the sense of operator-valued distributions. One has in particular (see [16])

- $\int d x f(x) A(x)=0$ if $f \in \mathcal{S}\left(\mathbb{R}^{1+s}\right)$ satisfies $\operatorname{supp} \tilde{f} \cap \operatorname{sp} A=\emptyset$;

- $\operatorname{sp} A^{*}=-\operatorname{sp} A, \quad$ where $A^{*}(x)=(A(x))^{*}$;

- $\operatorname{sp}\left(A_{1}+A_{2}\right) \subset \operatorname{sp} A_{1} \cup \operatorname{sp} A_{2}$;

- $\operatorname{sp}\left(A_{1} A_{2}\right) \subset \operatorname{sp} A_{1}+\operatorname{sp} A_{2}$ if $\operatorname{sp} A_{1}$ or $\operatorname{sp} A_{2}$ is bounded.

These properties will enter into the proof of the following lemma. (No results in this spirit seem to have appeared in the literature; the present formulation is due to Buchholz.)

Lemma 4.3 Let $C$ be a closed subset of $\mathbb{R}^{1+s}$. Then one has $s(\Gamma) \subset C$ iff

$$
\operatorname{sp}\left(\Gamma(\cdot) E_{I}(D)\right) \subset C-D \quad \text { for all compact sets } D \subset \mathbb{R}^{1+s} .
$$

Proof: Let $s(\Gamma) \subset C$. For any compact set $D \subset \mathbb{R}^{1+s}$, one then has

$$
\operatorname{sp}\left(\Gamma(\cdot) E_{I}(D)\right)=\operatorname{sp}\left(V_{\Gamma}(\cdot) U_{I}^{*}(\cdot) E_{I}(D)\right) \subset \operatorname{sp} V_{\Gamma}+\operatorname{sp}\left(U_{I}^{*}(\cdot) E_{I}(D)\right) .
$$

But $\operatorname{sp} V_{\Gamma}=s(\Gamma) \subset C$ and $\operatorname{sp}\left(U_{I}^{*}(\cdot) E_{I}(D)\right) \subset-D$, whence $\operatorname{sp}\left(\Gamma(\cdot) E_{I}(D)\right) \subset C-D$, showing the "only if" part of the assertion. To prove the converse, assume that $\Gamma$ fulfils (4.1). Let $D \subset \mathbb{R}^{1+s}$ be compact and choose a cover of $D$ by a finite number of pairwise disjoint Borel sets $\left(D_{j}\right)_{j=1, \ldots, N}$. By the properties of the spectrum noticed above, one then gets

$$
\begin{aligned}
\operatorname{sp}\left(V_{\Gamma}(\cdot) E_{I}(D)\right) & \subset \operatorname{sp}\left(V_{\Gamma}(\cdot) \sum_{j} E_{I}\left(D_{j}\right)\right)=\operatorname{sp} \sum_{j}\left(\Gamma(\cdot) E_{I}\left(D_{j}\right) E_{I}\left(D_{j}\right) U_{I}(\cdot)\right) \\
& \subset \bigcup_{j}\left(\operatorname{sp}\left(\Gamma(\cdot) E_{I}\left(D_{j}\right)\right)+\operatorname{sp}\left(E_{I}\left(D_{j}\right) U_{I}(\cdot)\right)\right) \\
& \subset \bigcup_{j}\left(C-\overline{D_{j}}+\overline{D_{j}}\right)=C+\bigcup_{j}\left(\overline{D_{j}}-\overline{D_{j}}\right) .
\end{aligned}
$$

Since the cover may be chosen such that the maximal diameter of the sets $\overline{D_{j}}$ is arbitrarily small, this implies $\operatorname{sp}\left(V_{\Gamma}(\cdot) E_{I}(D)\right) \subset C$ for any compact $D \subset \mathbb{R}^{1+s}$. In view of $\mathrm{s}^{-\lim _{D} / \mathbb{R}^{1+s}} E_{I}(D)=\mathbf{1}_{\mathcal{H}_{I}}$, this yields $s(\Gamma)=\operatorname{sp} V_{\Gamma}(\cdot) \subset C$. 


\subsection{Spectra of Pointlike Charges}

We will now discuss the spectral properties of pointlike charges and thus assume that $\mathcal{X}$ is the set of all double cones. What lies at the heart of the following is that the infravacuum representation $\pi_{I}$ is locally normal (see Lemma 2.3). In particular $\mathfrak{A}_{I}(\mathcal{O})$ is a von Neumann algebra, and the duality property of $\pi_{I}$ reads $\mathfrak{A}_{I}\left(\mathcal{O}^{\prime}\right)^{\prime}=\mathfrak{A}_{I}(\mathcal{O})$. As is well known, this implies that the $\mathrm{C}^{*}$-category $\Delta$ is a subcategory of End $\mathfrak{A}_{I}$ (Lemma 3.2). It can be made into a symmetric $\mathrm{rC}^{*}$-category $(\Delta, \epsilon)$ without an explicit introduction of the (directed!) net of its local subcategories $\mathcal{O} \longmapsto \Delta(\mathcal{O})$ of which it is the inductive limit.

Let us now assume that not only $\pi_{I}$ but also the vacuum representation $\pi_{0}$ is a valid background for superselection theory. (Since Property B for $\pi_{I}$ and for $\pi_{0}$ are equivalent due to local normality, this only amounts to assuming duality for $\pi_{0}$.) Then one has two symmetric $\mathrm{rC}^{*}$-categories $\left(\Delta^{(0)}, \epsilon^{(0)}\right)$ and $\left(\Delta^{(I)}, \epsilon^{(I)}\right)$ which describe the superselection theories in front of the vacuum and in front of the infravacuum, respectively. (The notations $\Delta_{\mathrm{f}}^{\#}, \Delta_{\mathrm{f}, \mathrm{c}}^{\#}, I^{\#}(\sigma, \tau), \beta_{x}^{\#}, Z^{\#}(\rho), Z_{0}^{\#}(\rho)$ with $\# \in\{(0),(I)\}$ should be self-explanatory. $)$

A priori these categories are different, but the following proposition shows that they actually contain the same information. Its proof is based on the fact that, as the algebra $\mathfrak{A}_{0}$ is simple, the homomorphism $\varphi:=\pi_{I} \circ \pi_{0}^{-1}: \mathfrak{A}_{0} \longrightarrow \mathfrak{A}_{I}$ is bijective and defines a net isomorphism (i.e., satisfies $\left.\varphi(\mathfrak{A}(\mathcal{O}))=\mathfrak{A}_{I}(\mathcal{O})\right)$.

\section{Proposition 4.4}

i. Let $\Delta^{(0)}$ and $\Delta^{(I)}$ be as above. Then a bijective monoidal functor $F: \Delta^{(0)} \longrightarrow \Delta^{(I)}$ is defined (on objects $\rho \in \Delta^{(0)}$ resp. on morphisms $t \in I^{(0)}(\sigma, \tau)$ ) by

$$
\begin{aligned}
\rho \longmapsto F(\rho) & :=\varphi \circ \rho \circ \varphi^{-1}, \\
t \longmapsto F(t) & :=(F(\tau), \varphi(t), F(\sigma)) .
\end{aligned}
$$

This functor respects the symmetries and restricts to a bijection $F: \Delta_{\mathrm{f}}^{(0)} \longrightarrow \Delta_{\mathrm{f}}^{(I)}$ between the subcategories of finite dimensional objects.

ii. The functor $F$ maps the covariant objects in $\Delta_{\mathrm{f}}^{(0)}$ onto the covariant ones in $\Delta_{\mathrm{f}}^{(I)}$. For each pair of corresponding covariant objects, it induces a bijection between the sets of general and of natural charge transporting cocycles.

Proof: i. Being an isomorphism of $\mathrm{C}^{*}$-algebras, $\varphi: \mathfrak{A}_{0} \longrightarrow \mathfrak{A}_{I}$ defines a monoidal isofunctor $F: \operatorname{End}_{0} \longrightarrow \operatorname{End}_{I}$. Since $\varphi$ is a net isomorphism, it follows that $F(\rho)$ is localised in $\mathcal{O}$ iff $\rho$ is. As a consequence, the transportability of $F(\rho)$ is equivalent to that of $\rho$, and one has $F\left(\Delta^{(0)}(\mathcal{O})\right)=\Delta^{(I)}(\mathcal{O})$. In particular $F$ is an isomorphism between the two symmetric monoidal categories $\Delta^{(0)}$ and $\Delta^{(I)}$ (cf. Prop. 3.13, i for the symmetries).

ii. The map between $Z^{(0)}(\rho)$ and $Z^{(I)}(F(\rho))$ is of course given by $(\Gamma(x))_{x \in \mathbb{R}^{1+s}} \longmapsto$ $(F(\Gamma(x)))_{x \in \mathbb{R}^{1+s}}$. In view of Part $\mathrm{i}$ and because one has $F \circ \beta_{x}^{(0)}=\beta_{x}^{(I)} \circ F$, the only subtle point is to verify that $F$ respects the continuity properties. But this is indeed the case: Given $\rho \in \Delta_{\mathrm{f}, \mathrm{c}}^{(0)}$, a point $x \in \mathbb{R}^{1+s}$ and some compact neighbourhood $K$ of $x$, there is always a double cone $\mathcal{O}$ such that $\Gamma(y) \in \mathfrak{A}(\mathcal{O})$ and $\varphi(\Gamma(y)) \in \mathfrak{A}_{I}(\mathcal{O})$ for all $y \in K$. Therefore due to Part iii of Lemma 2.3, the continuity of $x \longmapsto \Gamma(x)$ is equivalent to that of $x \longmapsto F(\Gamma(x))$.

Notice that the spectral properties of $U_{0}$ and $U_{I}$ have not been used for this result; it 
remains valid for any pair of background representations (i.e., fulfilling $\mathbf{b} \mathbf{1}$ and $\mathbf{b 2}$ ) which are locally normal to each other.

Notation: It is useful to write $\rho^{(0)}$ and $\rho^{(I)}$ for a pair of objects of $\Delta^{(0)}$ and $\Delta^{(I)}$ related by $\rho^{(I)}=F\left(\rho^{(0)}\right)$. Similarly, we denote with $\Gamma^{(0)} \in Z^{(0)}\left(\rho^{(0)}\right)$ and $\Gamma^{(I)} \in Z^{(I)}\left(\rho^{(I)}\right)$ a pair of cocycles satisfying $\Gamma^{(I)}(x)=F\left(\Gamma^{(0)}(x)\right)$.

The next natural question is how the spectra of $\Gamma^{(0)}$ and $\Gamma^{(I)}$ are related. This is the point where the property of $\pi_{I}$ being an infravacuum representation enters. We have seen in Prop. 2.4 that under reasonable physical assumptions the nets $\tilde{\mathcal{S}}_{\pi_{0}}(\cdot)$ and $\tilde{\mathcal{S}}_{\pi_{I}}(\cdot)$ of states are closely related to each other. This relation is used in the following proposition. The idea for its proof is due to Buchholz.

Proposition 4.5 Let the nets $\tilde{\mathcal{S}}_{\pi_{0}}(\cdot)$ and $\tilde{\mathcal{S}}_{\pi_{I}}(\cdot)$ of states fulfil the two inclusions of Prop. 2.4. Then any pair of cocycles $\Gamma^{(0)} \in Z^{(0)}\left(\rho^{(0)}\right)$ and $\Gamma^{(I)} \in Z^{(I)}\left(\rho^{(I)}\right)$ related by F satisfies

$$
s\left(\Gamma^{(I)}\right)=s\left(\Gamma^{(0)}\right) .
$$

Proof: We will show the inclusion " $\subset$ " using the characterisation of the spectra given in Lemma 4.3. Let $D \subset \mathbb{R}^{1+s}$ be compact and let $D_{0}$ be any compact neighbourhood of $D$. From Prop. 2.4 one has $\tilde{\mathcal{S}}_{\pi_{I}}(D) \subset \tilde{\mathcal{S}}_{\pi_{0}}\left(D_{0}\right)$. Now choose a test function $f$ satisfying $\operatorname{supp} \tilde{f} \cap\left(s\left(\Gamma^{(0)}\right)-D_{0}\right)=\emptyset$. Then $\operatorname{sp}\left(\Gamma^{(0)}(\cdot) E_{0}\left(D_{0}\right)\right) \subset s\left(\Gamma^{(0)}\right)-D_{0}$ implies for $\Gamma^{(0)}(f):=\int d x f(x) \Gamma^{(0)}(x)$ that $\Gamma^{(0)}(f) E_{0}\left(D_{0}\right)=0$. Therefore $\omega\left(\pi_{0}^{-1}\left(\Gamma^{(0)}(f)^{*} \Gamma^{(0)}(f)\right)\right)=$ 0 for any vector state $\omega \in \mathcal{S}_{\pi_{0}}\left(D_{0}\right)$, hence (by weak continuity) for any $\omega \in \tilde{\mathcal{S}}_{\pi_{0}}\left(D_{0}\right)$ and in particular for any $\omega \in \tilde{\mathcal{S}}_{\pi_{I}}(D)$. We thus have $\varphi\left(\Gamma^{(0)}(f)\right) E_{I}(D)=0$. By the local normality of $\varphi$, one has $\varphi\left(\Gamma^{(0)}(f)\right)=\Gamma^{(I)}(f)$, which implies $\Gamma^{(I)}(f) E_{I}(D)=0$. As $f$ was arbitrary, this means $\operatorname{sp}\left(\Gamma^{(I)}(\cdot) E_{I}(D)\right) \subset s\left(\Gamma^{(0)}\right)-D_{0}$. Now $D_{0}$ may be any neighbourhood of $D$, and since $D$ itself was arbitrary, Lemma 4.3 yields $s\left(\Gamma^{(I)}\right) \subset s\left(\Gamma^{(0)}\right)$. The converse inclusion is proved similarly.

The last two propositions show that in the case of pointlike charges the superselection structure can be described equally well in front of the vacuum as in front of any infravacuum. Exactly the same charges are seen in front of both backgrounds; their fusion structure and their statistics are the same. More importantly, also the spectral properties of these charges are unaffected. This can be interpreted by saying that the background has a very weak influence on the charged particles and their interaction. It excludes for instance effects which might yield some background-dependent "effective" masses.

\subsection{The Role of the Vacuum Vector}

In Section 4.1 we have already cited the most important properties of the spectra $s(\rho)$ for charges in front of the vacuum, and we have seen in the previous section that they carry over to any infravacuum background if the charges are compactly localised. This was a rather trivial consequence of local normality; the nontrivial part of the input was hidden in the inclusions of between the nets $\tilde{\mathcal{S}}_{\pi_{0}}(\cdot)$ and $\tilde{\mathcal{S}}_{\pi_{I}}(\cdot)$ of states.

For non-compactly localised charges a similar situation will not be present. It is therefore of interest how an alternative proof of the properties in question can be obtained which does not rely on the existence of a translation invariant vector. To this end it is instructive to analyse exactly at which points this invariance is used, and we thus want to recall the main points of the proof of Prop. 4.2, following [ $[8,9]$. 
In a first step, the invariance of the vacuum vector together with the cluster property is used to deduce a property called the additivity of the spectra. In our formulation in terms of charge transporting cocycles it reads as follows:

add Whenever $\Gamma_{j} \in Z_{0}\left(\rho_{j}\right), j=1,2$ are cocycles for irreducible objects $\rho_{j} \in \Delta(X)_{\mathrm{f}, \mathrm{c}}$, then one has $s\left(w^{*}\left(\Gamma_{1} \otimes \Gamma_{2}\right)\right) \supset s\left(\Gamma_{1}\right)+s\left(\Gamma_{2}\right)$ for any isometry $w: \sigma \hookrightarrow \rho_{1} \rho_{2}$ which projects $\Gamma_{1} \otimes \Gamma_{2}$.

(The formal condition on $w$ is necessary at this stage since it is not yet clear that $\Gamma_{1} \otimes \Gamma_{2} \in$ $Z_{0}\left(\rho_{1} \rho_{2}\right)$. This will however follow from Lemma 4.6.)

In the special case of $\iota \subset \bar{\rho} \rho$ (cf. Lemma 3.22) the property add implies for any (irreducible) object $\rho \in \Delta(X)_{\mathrm{f}, \mathrm{c}}$ and any cocycle $\Gamma \in Z(\rho)=Z_{0}(\rho)$ that

$$
\overline{\mathbf{V}}_{+} \supset s(\bar{\Gamma})+s(\Gamma) \text {. }
$$

Since the spectra are nonempty, this means $s(\Gamma) \cup s(\bar{\Gamma}) \subset \overline{\mathbf{V}}_{+}-q$ for some $q \in \mathbb{R}^{1+s}$ and thus shows that $\rho$ and $\bar{\rho}$ have positive energy, which is Part i of Prop. 4.2. The minimal cocycles $\Gamma_{\rho}$ and $\Gamma_{\bar{\rho}}$ are thus well-defined, and since $\bar{\rho}$ is irreducible, one has $\overline{\Gamma_{\rho}}(x)=e^{i Q x} \Gamma_{\bar{\rho}}(x)$ for some $Q \in \mathbb{R}^{1+s}$. Inserting $\Gamma_{\rho}$ into equation (4.2) now yields

$$
\overline{\mathbf{V}}_{+} \supset s\left(\overline{\Gamma_{\rho}}\right)+s\left(\Gamma_{\rho}\right)=Q+s(\bar{\rho})+s(\rho),
$$

which shows that $Q \in \overline{\mathbf{V}}_{+}$since the lower boundaries of $s(\rho)$ and $s(\bar{\rho})$ are Lorentz-invariant.

In a second step the invariance of the vacuum vector is used again (together with the net structure of $\mathfrak{A}_{0}$ and the information $\left.Q \in \overline{\mathbf{V}}_{+}\right)$for proving the relation $s\left(\overline{\Gamma_{\rho}}\right)=s\left(\Gamma_{\rho}\right)$. It implies in particular that $Q=0$, whence $\overline{\Gamma_{\rho}}=\Gamma_{\bar{\rho}}$ and therefore yields $s(\rho)=s(\bar{\rho})$, i.e., Part ii of Prop. 4.2. We do not comment on this step further here since we will show in the next section how $\overline{\Gamma_{\rho}}=\Gamma_{\bar{\rho}}$ (albeit not $s(\rho)=s(\bar{\rho})$ ) could as well be obtained at this stage without using the invariance of the vacuum vector.

Finally, Part iii of Prop. 4.2 is a direct consequence of the fact that the natural family $\left(\Gamma_{\rho}\right)_{\rho \in \Delta_{\mathrm{f}, \mathrm{c}}}$ of the minimal cocycles is monoidal. As will be shown in the following lemma, this in turn relies on $\overline{\Gamma_{\rho}}=\Gamma_{\bar{\rho}}$ (which is easily seen to carry over to reducible objects) and on the additivity property add.

Lemma 4.6 Assume that the additivity property add holds and that the minimal cocycles satisfy $\overline{\Gamma_{\rho}}=\Gamma_{\bar{\rho}}$. For any $\rho_{1}, \rho_{2} \in \Delta(X)_{\mathrm{f}, \mathrm{c}}$, one then has $\Gamma_{\rho_{1}} \otimes \Gamma_{\rho_{2}}=\Gamma_{\rho_{1} \rho_{2}}$.

Proof: Let $\Gamma_{0} \in Z_{0}\left(\rho_{1} \rho_{2}\right)$ be the minimal cocycle for $\rho_{1} \rho_{2}$. Then there exists by Lemma 3.23 a continuous unitary representation $B: \mathbb{R}^{1+s} \longrightarrow I\left(\rho_{1} \rho_{2}, \rho_{1} \rho_{2}\right)$ satisfying $\left(\Gamma_{\rho_{1}} \otimes \Gamma_{\rho_{2}}\right)(x)=B(x) \Gamma_{0}(x)$. From spectral theory it follows that $B(x)=\sum_{j} e^{i Q_{j} x} E_{j}$ with a complete family of 1-dimensional projections $E_{j} \in I\left(\rho_{1} \rho_{2}, \rho_{1} \rho_{2}\right)$ and vectors $Q_{j} \in \mathbb{R}^{1+s}, j=1, \ldots, J$. Then any family $w_{j}: \sigma_{j} \hookrightarrow \rho_{1} \rho_{2}$ of isometries with final projections $E_{j}$ performs a decomposition of $\rho_{1} \rho_{2}=\bigoplus \sigma_{j}$ into irreducible subobjects $\sigma_{j}$, and each of these isometries projects $\Gamma_{\rho_{1}} \otimes \Gamma_{\rho_{2}}$, yielding $w_{j}^{*}\left(\Gamma_{\rho_{1}} \otimes \Gamma_{\rho_{2}}\right)(x)=e^{i Q_{j} x} \Gamma_{\sigma_{j}}(x)$. Due to the additivity property add one has $Q_{j}+s\left(\sigma_{j}\right) \supset s\left(\rho_{1}\right)+s\left(\rho_{2}\right)$, and in particular $Q_{j} \in \overline{\mathbf{V}}_{-}$. On the other hand, one obtains (under the conjugation $\dagger$ ) from the above one the identity $w_{j}^{\dagger *}\left(\overline{\Gamma_{\rho_{2}}} \otimes \overline{\Gamma_{\rho_{1}}}\right)(x)=e^{-i Q_{j} x} \overline{\Gamma_{\sigma_{j}}}(x)$. Because of $\overline{\Gamma_{\rho}}=\Gamma_{\bar{\rho}}$ this reads $w_{j}^{\dagger *}\left(\Gamma_{\bar{\rho}_{2}} \otimes \Gamma_{\bar{\rho}_{1}}\right)(x)=e^{-i Q_{j} x} \Gamma_{\bar{\sigma}_{j}}(x)$. Property add (for $w_{j}^{\dagger}: \bar{\sigma}_{j} \hookrightarrow \bar{\rho}_{2} \bar{\rho}_{1}$ this time) now implies by the same reasoning that $-Q_{j} \in \overline{\mathbf{V}}_{-}$. Taken together, this means $Q_{j}=0$, so $B$ is trivial and $\Gamma_{\rho_{1}} \otimes \Gamma_{\rho_{2}}$ is minimal. 


\subsection{The Minimal Cocycles under Conjugation}

In the previous section we have recalled how Prop. 4.2 is derived in the case of charges in front of the vacuum. The invariance of the vacuum vector enters twice into that proof, namely first into the additivity property add and second into the proof of the relation $s\left(\overline{\Gamma_{\rho}}\right)=s\left(\Gamma_{\rho}\right)$. The latter implies in particular that the minimal cocycles are invariant under conjugation, i.e.,

$$
\overline{\Gamma_{\rho}}=\Gamma_{\bar{\rho}} .
$$

This is necessary if the minimal family of cocycles is to be monoidal, and it is even sufficient if add is known.

In this section we consider superselection charges with spacelike cone localisation in front of an infravacuum background. Assuming (4.2), which is a special case of add, we will show that in this case (4.3) can be obtained from the property $\operatorname{sp} U_{I}=\overline{\mathbf{V}}_{+}$only. The latter serves as a substitute for the presence of a truly invariant vector, since it implies that there are vectors in $\mathcal{H}_{I}$ which vary arbitrarily slowly under translations.

In just postulating the additivity of the energy, we regard this condition as a (strong) form of the assumption that the infravacuum background does not affect the energymomentum properties of the charges, i.e., in a certain sense as a strengthening of the tight relation (found in Prop. 2.4) between the nets $\tilde{\mathcal{S}}_{\pi_{0}}(\cdot)$ and $\tilde{\mathcal{S}}_{\pi_{I}}(\cdot)$ of states. It would be highly desirable to derive it from suitable assumptions in the spirit of this relation, that is, by exploiting the idea that an infravacuum representation is "energetically close" to the vacuum. Alas no such result is presently available.

Stated precisely, we assume $\mathcal{X}$ to be the set of all spacelike cones and $\pi_{I}$ to be a background representation fulfilling b1 (Property B), b2 (duality) and to be such that $\operatorname{sp} U_{I}=\overline{\mathbf{V}}_{+}$. We then prove:

Proposition 4.7 Let $\rho, \bar{\rho} \in \Delta(X)_{\mathrm{f}, \mathrm{c}}$ be irreducible objects conjugate to each other and $\Gamma \in Z(\rho)$. Assume that $s(\bar{\Gamma})+s(\Gamma) \subset \overline{\mathbf{V}}_{+}$. Then $\rho$ and $\bar{\rho}$ have positive energy, and one has $\overline{\Gamma_{\rho}}=\Gamma_{\bar{\rho}}$.

The first steps of the proof are exactly as in Section 4.3; we thus already know that $\overline{\Gamma_{\rho}}(x)=e^{i Q x} \Gamma_{\bar{\rho}}(x)$ for some vector $Q \in \overline{\mathbf{V}}_{+}$. The aim of obtaining $Q=0$ will now be reached by showing $Q \in \overline{\mathbf{V}}_{-}$(or, more precisely, by constructing a contradiction from the hypothesis $Q \notin \overline{\mathbf{V}}_{-}$).

Remark: The main technical problem is to deal with the case $Q^{2}=0$. If we knew $Q \in\{0\} \cup \mathbf{V}_{+}$rather than $Q \in \overline{\mathbf{V}}_{+}$- for instance, if $\rho$ and $\bar{\rho}$ were rotation covariant in some Lorentz frame - , then we could derive $Q=0$ from $\operatorname{sp} U_{I}=\overline{\mathbf{V}}_{+}$along the classical lines of $[\boldsymbol{\varepsilon}]$ without the more general technique presented below.

Thus assume that $Q \notin \overline{\mathbf{V}}_{-}$. Then there exist vectors $q_{0} \in \mathbf{V}_{+}$and $q_{1} \in\left\{q_{0}\right\}^{\perp}$ such that $Q=4 q_{0}+q_{1}$ and a wedge region $W$ whose edge contains the subspace $\mathbb{R} q_{1}$. Moreover there exist spacelike cones $X_{1}, X_{2}, Y \in \mathcal{X}$ such that $X_{1} \cup X_{2} \subset Y$ and

$$
X_{1} \times\left(X_{2}+x\right) \quad \text { for every } x \in \bar{W} \text {. }
$$

Since the sets $s\left(\overline{\Gamma_{\rho}}\right)$ and $s\left(\Gamma_{\bar{\rho}}\right)$ and (as a consequence) the vector $Q$ are unitary invariants, it means no loss of generality to assume in addition that the localisation region $X$ of the objects $\rho$ and $\bar{\rho}$ is contained in $Y$. 
Remarks: 1. Although $q_{1}$ is in momentum space, the wedge $W$ is thought of as a subset of position space. A wedge region $W$ is by definition a (degenerate) open spacelike cone bounded by two lightlike (half) hyperplanes; its edge is the (spacelike, $(s-1)$ dimensional) affine subspace $\bar{W} \cap \overline{W^{\prime}}$. The above-mentioned condition on $W$ thus means that $W$ is invariant under the translations by $\mathbb{R} q_{1}$ and that the edge contains the origin. 2. The geometry of $Q, q_{0}, q_{1}$ and $W$ is easy to visualise in a Lorentz system where $Q$ has positive time component. In such a system, one can take $q_{0}$ along the 0 -axis, assume $q_{1}$ to be on the 1-axis and take for $W$ the wedge $\left\{\left(x^{\mu}\right)_{\mu=0, \ldots, s}|| x^{0} \mid<x^{2}\right\}$.

3 . It is essential that $q_{0}$ is contained in the interior of the forward light cone.

4. We show the existence of the spacelike cones $X_{1}$ and $X_{2}$ in the following separate lemma which allows for the additional constraint that only upright spacelike cones in some given Lorentz system are available. Notice that this lemma would fail in the case of the opposite spacelike cones introduced in Section 3.2.

Lemma 4.8 Let $W$ be a wedge region and let $e \in \mathbf{V}_{+}, e^{2}=1$ define a Lorentz system. Then there exist upright spacelike cones $X_{1}, X_{2}$ and $Y$ satisfying $X_{1} \cup X_{2} \subset Y$ and $X_{1} \times\left(X_{2}+\bar{W}\right)$.

Proof: It is convenient to choose coordinates such that $0 \in \bar{W} \cap \overline{W^{\prime}}$ and $e=(1, \overrightarrow{0})$. The wedge region $W$ has the general form $W=\left\{x \in \mathbb{R}^{1+s} \mid k_{ \pm} x<0\right\}$ for some vectors $k_{ \pm}=\left( \pm 1, \vec{n}_{ \pm}\right)$; here $\vec{n}_{ \pm} \in \mathbb{R}^{s}$ are two unit vectors not opposite to each other. The wedge $W$ intersects the time 0 hyperplane $\{e\}^{\perp}=\{0\} \times \mathbb{R}^{s}$ in the set $W \cap\{e\}^{\perp}=\{0\} \times C$, where $C=\left\{\vec{x} \in \mathbb{R}^{s} \mid \vec{n}_{ \pm} \vec{x}>0\right\}$. Since $C$ is an open convex cone, there exists some open (pointed) circular cone $S$ which intersects both $C$ and $-C$, and there exist some open (pointed) circular cones $S_{1} \subset-C \cap S$ and $S_{2} \subset C \cap S$. Setting $X_{j}:=\left(\{0\} \times S_{j}\right)^{\prime \prime}$ and $Y:=(\{0\} \times S)^{\prime \prime}$, one obtains upright spacelike cones which satisfy $X_{1} \cup X_{2} \subset Y$. Now $\{0\} \times S_{2} \subset\{0\} \times C \subset W$ implies $X_{2} \subset W$ and therefore $X_{2}+\bar{W} \subset \bar{W}$ (since $\bar{W}$ is convex), whereas $\{0\} \times S_{1} \subset\{0\} \times(-C) \subset-W=\bar{W}^{\prime}$ yields $X_{1} \subset \bar{W}^{\prime}$. Taken together, this means $X_{1} \subset\left(X_{2}+\bar{W}\right)^{\prime}$, as desired.

Starting from the above geometrical data (induced by the hypothetical position of $Q$ ), choose objects $\sigma_{j} \in \Delta\left(X_{j}\right)$ and arbitrary morphisms $t_{j}: \sigma_{j} \rightarrow \rho$. With the notations

$$
V(x):=\Gamma_{\rho}(x) U_{I}(x) \quad \text { and } \quad \bar{V}(x):=\overline{\Gamma_{\rho}}(x) U_{I}(x)
$$

one then has the following identity in $\mathcal{B}\left(\mathcal{H}_{I}\right)$, proved in Appendix C:

$$
t_{1}^{*} V(x) t_{2} U_{I}(x)^{*}=\kappa U_{I}(x)\left(\bar{\rho}^{Y}\left(t_{2}^{*}\right) r\right)^{*} \bar{V}(x)^{*}\left(\bar{\rho}^{Y}\left(t_{1}^{*}\right) r\right) \quad \text { for every } x \in \bar{W} \text {. }
$$

Here $\bar{\rho}^{Y}$ is the extension of $\bar{\rho}$ to $\mathfrak{A}_{I}(Y)^{\prime \prime}$, say (cf. Lemma 3.4),$\kappa \in\{ \pm 1\}$ is the statistical phase of $\rho$ and $\bar{\rho}$, and $(r, \bar{r}) \in \mathcal{R}(\rho, \bar{\rho})$ is some standard solution of the conjugate equations.

Let now $\Phi_{j} \in \mathcal{H}_{I}(j=1,2)$ be two arbitrary vectors and define the continuous functions $f_{ \pm}: \mathbb{R}^{1+s} \longrightarrow \mathbb{C}$ by

$$
\begin{aligned}
& f_{+}(x):=\left\langle V(x)^{*} t_{1} \Phi_{1}, t_{2} U_{I}(x)^{*} \Phi_{2}\right\rangle \\
& f_{-}(x):=\kappa\left\langle\bar{\rho}^{Y}\left(t_{2}^{*}\right) r U_{I}(x)^{*} \Phi_{1}, \bar{V}(x)^{*} \bar{\rho}^{Y}\left(t_{1}^{*}\right) r \Phi_{2}\right\rangle
\end{aligned}
$$


The identity (4.4) implies that the function

$$
f: \mathbb{R}^{1+s} \longrightarrow \mathbb{C}: x \longmapsto f(x):=f_{+}(x)-f_{-}(x)
$$

vanishes in the wedge region $W$. This information will be combined with the momentum space properties of $f$ in order to show that much more is true, namely that both $f_{+}$and $f_{-}$vanish identically in all of $\mathbb{R}^{1+s}$ if the vectors $\Phi_{j}$ are chosen appropriately. (If there

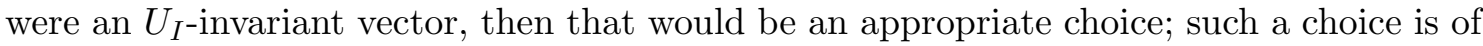
course impossible here, but we will see that vectors whose energy-momentum support are close enough to the origin are still good enough.)

We begin by describing how the momentum space properties of the functions $f_{ \pm}$depend on the vectors $\Phi_{j}$. Notice that $f_{ \pm}$are continuous bounded functions, hence their Fourier transforms $\hat{f}_{ \pm}$exist in the sense of tempered distributions. In the sequel, we call the spectrum of such a function (which may also be vector- or operator-valued) the support of its Fourier transform. In the case of a strongly continuous unitary group this coincides of course with the common spectrum of its generators.

Lemma 4.9 Let $D_{j} \subset \overline{\mathbf{V}}_{+}$be compact sets $(j=1,2)$. If $\Phi_{j} \in E_{I}\left(D_{j}\right) \mathcal{H}_{I}$, then one has

$$
\operatorname{supp} \hat{f}_{+} \subset s(\rho)-D_{2}, \quad \operatorname{supp} \hat{f}_{-} \subset-Q-s(\bar{\rho})+D_{1} .
$$

Proof: The spectrum of $x \longmapsto V(x)=\Gamma_{\rho}(x) U_{I}(x)$ is $s(\rho)$. Therefore the (uniformly continuous bounded vector-valued) function $x \longmapsto V(x)^{*} \Psi$ has (for any $\Psi \in \mathcal{H}_{I}$, and in particular for $\left.\Psi=t_{1} \Phi_{1}\right)$ its spectrum in $-s(\rho)$; on the other hand $x \longmapsto B U_{I}(x)^{*} \Phi_{2}$ has (for any $B \in \mathcal{B}\left(\mathcal{H}_{I}\right)$, and especially for $B=t_{2}$ ) its spectrum in $-D_{2}$. For the (pointwise) scalar product $f_{+}$of these functions, this means $\operatorname{supp} \hat{f}_{+} \subset s(\rho)-D_{2}$.

Similar arguments apply to the case of $f_{-}$; the main difference is that the spectrum of $x \longmapsto \bar{V}(x)=e^{i Q x} \Gamma_{\bar{\rho}}(x) U_{I}(x)$ is $Q+s(\bar{\rho})$.

Let us now choose $\Phi_{j} \in E_{I}\left(D_{q_{0}}\right) \mathcal{H}_{I}$, where $D_{q_{0}}:=\overline{\mathbf{V}}_{+} \cap\left(q_{0}+\overline{\mathbf{V}}_{-}\right)$. Then Lemma 4.9 implies

$$
\begin{aligned}
& \operatorname{supp} \hat{f}_{+} \subset s(\rho)-D_{q_{0}} \subset-q_{0}+\overline{\mathbf{V}}_{+}, \\
& \operatorname{supp} \hat{f}_{-} \subset-Q-s(\bar{\rho})+D_{q_{0}} \subset q_{0}-Q+\overline{\mathbf{V}}_{-} .
\end{aligned}
$$

Setting $a_{+}:=-q_{0}$ and $a_{-}:=q_{0}-Q$, this reads supp $\hat{f}_{ \pm} \subset\left(a_{ \pm}+\overline{\mathbf{V}}_{ \pm}\right)$, and we thus have

$$
\operatorname{supp} \hat{f} \subset G:=\left(a_{+}+\overline{\mathbf{V}}_{+}\right) \cup\left(a_{-}+\overline{\mathbf{V}}_{-}\right) \quad \text { and }\left.\quad f\right|_{W}=0 \text {. }
$$

The apices of the two components of $G$ are separated by $a_{+}-a_{-}=Q-2 q_{0}=2 q_{0}+q_{1}$. As this vector need not be in $\mathbf{V}_{+}$(assuming $q_{1} \neq 0$ ), one cannot apply the so-called Jost-Lehmann-Dyson method to $f$ in order to obtain $f=0$. However the intersection of $G$ with any hyperplane perpendicular to $q_{1}$ (i.e., of the form $\mathcal{M}_{\xi}:=\xi q_{1}+\left\{q_{1}\right\}^{\perp}$ with $\xi \in \mathbb{R}$ ) does consist of two components separated by the timelike vector $2 q_{0}$. Now the restriction of $\hat{f}$ to $\mathcal{M}_{\xi}$ is the Fourier transform of a "function" $f_{\xi}$ in the $s$-dimensional Minkowski space $\mathbb{R}^{1+s} /\left(\mathbb{R} q_{1}\right) \cong \mathbb{R}^{1+(s-1)}$ which arises from $f$ by multiplication with a phase ( $x \longmapsto e^{i \xi q_{1} x}$ to be precise) and subsequent integration along the $q_{1}$-direction. But the wedge $W$ is invariant under the translations in $\mathbb{R} q_{1}$, so $\left.f\right|_{W}=0$ implies that $f_{\xi}$ vanishes in the projected wedge $W /\left(\mathbb{R} q_{1}\right)$. By applying the Jost-Lehmann-Dyson method to this lower-dimensional situation, one can therefore conclude that $f_{\xi}=0$, and since this works for all $\xi \in \mathbb{R}$, it follows that $f$ vanishes identically. 
Remark: The above heuristic argument would work as it stands if $f$ were e.g. a test function. Nevertheless, the result is still true in present case of distributions, but since its proof requires some preparations, we postpone it to Appendix D. The exact statement relevant here is that of Prop. D.6. The main technical tool will be to fatten the hyperplane $\mathcal{M}_{\xi}$ in the $q_{1}$-direction, and we will even obtain some additional generality by replacing $\mathcal{M}_{\xi}$ with suitable lower-dimensional timelike planes.

Thus, we have the following result:

Lemma 4.10 If $\Phi_{j} \in E_{I}\left(D_{q_{0}}\right) \mathcal{H}_{I}$, then $f_{+}(x)=0$ and $f_{-}(x)=0$ for all $x \in \mathbb{R}^{1+s}$.

Proof: By combining Prop. D.6 with the properties of $f$ listed in (4.5), one has $f=0$. Thus $\hat{f}_{+}=\hat{f}_{-}$, and since the sets supp $\hat{f}_{ \pm}$are contained in the two disjoint components of $G$, this implies $\hat{f}_{+}=0$ and $\hat{f}_{-}=0$ separately. This proves the assertion.

The desired contradiction is now obtained in a standard manner: Lemma 4.10 means in particular that

$$
0=f_{+}(0)=\left\langle t_{1} \Phi_{1}, t_{2} \Phi_{2}\right\rangle
$$

for all vectors $\Phi_{j} \in E_{I}\left(D_{q_{0}}\right) \mathcal{H}_{I}$ and all $t_{j} \in I\left(\sigma_{j}, \rho\right)$ with arbitrary $\sigma_{j} \in \Delta\left(X_{j}\right)$. Now the set of operators $t_{j}$ fitting this description is invariant under multiplication from the right by unitaries $V_{j} \in \mathfrak{A}_{I}\left(X_{j}\right)^{\prime \prime}$ (because $t_{j} \in I\left(\sigma_{j}, \rho\right)$ with $\sigma_{j} \in \Delta\left(X_{j}\right)$ implies $t_{j} V_{j} \in I\left(\tilde{\sigma}_{j}, \rho\right)$ with $\left.\tilde{\sigma}_{j}:=\operatorname{Ad} V_{j}^{*} \cdot \sigma_{j} \in \Delta\left(X_{j}\right)\right)$, whence one has

$$
\left\langle t_{1} V_{1} \Phi_{1}, t_{2} V_{2} \Phi_{2}\right\rangle=0
$$

for all $t_{j}$ and $\Phi_{j}$ as above and arbitrary $V_{j} \in \mathfrak{A}_{I}\left(X_{j}\right)^{\prime \prime}$. (The latter need not be unitary any more since each element of $\mathfrak{A}_{I}\left(X_{j}\right)^{\prime \prime}$ is a linear combination of four unitaries.) On the other hand the assumption $\operatorname{sp} U_{I}=\overline{\mathbf{V}}_{+}$implies that the vectors $\Phi_{j} \in E_{I}\left(D_{q_{0}}\right) \mathcal{H}_{I}$ can be chosen nonzero, in which case they are cyclic for the algebras $\mathfrak{A}_{I}\left(X_{j}\right)^{\prime \prime}$. (Notice that as $X_{j}$ is a spacelike cone and therefore contains arbitrary large bounded regions, the cyclicity of each vector analytic for the energy does not need weak additivity of the representation $\pi_{I}$, cf. [17].) If in addition the morphisms $t_{j}$ are chosen to be unitary, then the sets $\left\{t_{j} V_{j} \Phi_{j} \mid V_{j} \in \mathfrak{A}_{I}\left(X_{j}\right)^{\prime \prime}\right\}$ are dense in $\mathcal{H}_{I}$. Therefore equation (4.6) means that $\mathcal{H}_{I}$ must be zero-dimensional, in obvious contradiction with the assumptions.

This establishes relation (4.3) and thus shows that the minimal cocycles are wellbehaved under charge conjugation. Obviously, this is necessary if the spectra $s\left(\Gamma_{\rho}\right)$ and $s\left(\overline{\Gamma_{\rho}}\right)$ are to be equal. From the physical point of view this ought to be the case if $\pi_{I}$ deserves the name of a background. Alas the method by which (4.3) was derived is not powerful enough to derive the equality of $s(\rho)$ and $s(\bar{\rho})$, so it remains as an open problem whether e.g. a particle and its antiparticle carrying charges with spacelike cone localisation in front an infravacuum necessarily have equal masses.

The formal reason for this difficulty is that if $Q=0$, then any two light cones of the form $a_{ \pm}+\overline{\mathbf{V}}_{ \pm}$enveloping supp $\hat{f}_{ \pm}$intersect in some set which contains an open neighbourhood of the origin (as long as the vectors $\Phi_{j}$ are not $U_{I}$-invariant), cf. Lemma 4.9. Therefore one cannot use Prop. D.3 any more for comparing as in [ [] ] the spectra of $f_{+}$and $f_{-}$. It is conceivable, however, that $s(\rho)=s(\bar{\rho})$ can actually be obtained in the present situation with more refined methods from the theory of functions of several complex variables. 


\section{Chapter 5}

\section{An Example for Background-Induced Localisation}

In the preceding chapters we have pointed out on several occasions that charges in theories with massless particles are expected to have better localisation properties in front of a suitably fluctuating background than in front of the vacuum. This idea has been put forward by Buchholz in [6], motivated by what is likely to happen in quantum electrodynamics. There the background states should correspond to clouds of infrared radiation. An appropriate mathematical description of such infrared clouds has been introduced by Kraus, Polley and Reents in [5].

Here we want to verify the localisation mechanism in one of the simplest conceivable models suited for that purpose, namely in the theory of the free massless scalar field in $(1+3)$-dimensional Minkowski space [34]. We will consider a certain class of (non-Lorentz invariant) sectors described by automorphisms of the observable algebra $\mathfrak{A}$, and we will show that these sectors have very poor localisation properties when seen in front of the vacuum, while they become localised in arbitrary upright spacelike cones when compared to a class of KPR-like background states. (Calling the background fields "KPR-like" should indicate that they are very similar, yet not identical, to those of [5].)

The free massless scalar field is of course much simpler than the physically interesting case of QED, but it shares with the latter the property that there is an analogue of Gauss' law by which the electric charge localised (in the naive sense) in a compact region of space (at every instant of time) is related to the flux of the asymptotic Coulomb field. In QED, the long-range degrees of freedom have no mutual interaction, and it is therefore legitimate to study effects related to them in a free theory.

The same sectors as those which will be studied below have been considered by Buchholz et al. in [34], and we will therefore stick quite closely to the notations introduced there. It has to be emphasised however that our point of view is different from the one adopted in [34]. Buchholz et al. achieve a better localisation by restricting the automorphisms to a (non-Lorentz invariant) subnet $\mathfrak{A}_{0} \subset \mathfrak{A}$ of the observable net. The sectors then even become localised in double cones, and the asymptotic commutation relations of the intertwining operators permit a DHR-like analysis to be carried through, compensating for the fact that the subnet $\mathfrak{A}_{0}$ does not fulfil duality. It therefore comes a bit as a disappointment that recent results of the same authors show that this way of improving the localisation of charged states is an artefact of free theories [35]. In physically realistic 
theories like QED, the simultaneous presence of interaction and of quantum effects entails that the distinction between the "charge support" and the "field support" (in the sense of localisation with respect to $\mathfrak{A}_{0}$ and $\mathfrak{A}$, respectively) of a charge cannot be maintained.

\subsection{The Model}

To begin with, let us recall the definition of the model under consideration. The observable algebra of the free massless scalar field is defined in its vacuum representation. More precisely, let $\mathcal{K}:=L^{2}\left(\mathbb{R}^{3}, d^{3} k\right)$ be the Hilbert space of momentum space wave functions, $\omega(\vec{k}):=|\vec{k}|$ the one-particle energy and $U(t, \vec{x})=e^{i(\omega(\vec{k}) t-\vec{k} \vec{x})}$ the usual representation of the spacetime translations. The vacuum Hilbert space of the model is the bosonic Fock space $\mathcal{H}$ over $\mathcal{K}$; the induced unitary representation of the translations will still be denoted by $U(t, \vec{x})$ without any risk of confusion. For any $v \in \mathcal{K}, W(v) \in \mathcal{B}(\mathcal{H})$ denotes the corresponding Weyl operator; the normalisation of $v$ is chosen such that the Weyl relations read $W(u) W(v)=e^{-\frac{i}{2} \operatorname{Im}\langle u, v\rangle} W(u+v)$. For any real linear subspace $\mathcal{L} \subset \mathcal{K}$ of the oneparticle space, $\mathcal{W}(\mathcal{L})$ denotes the $\mathrm{C}^{*}$-subalgebra of $\mathcal{B}(\mathcal{H})$ generated by the operators $W(u)$, $u \in \mathcal{L}$.

Like in any theory of a free bosonic field, the net of observables is best defined as the second quantisation of a net $\mathcal{O} \longmapsto \mathcal{L}(\mathcal{O})$ of symplectic subspaces of $\mathcal{K}$. Here this net is given as follows: If $\mathcal{O}:=(\{t\} \times O)^{\prime \prime}$ is the causal completion of an open ball $O \subset \mathbb{R}^{3}$ at time $t$, then

$$
\mathcal{L}(\mathcal{O}):=e^{i \omega t}\left(\omega^{-\frac{1}{2}} \widehat{\mathcal{D}_{\mathbb{R}}(O)}+i \omega^{+\frac{1}{2}} \widehat{\mathcal{D}_{\mathbb{R}}(O)}\right)
$$

where $\mathcal{D}_{\mathbb{R}}(O)$ is the set of all real-valued smooth functions with support in $O$ and ^ denotes the Fourier transform. For other open subsets $\mathcal{O} \subset \mathbb{R}^{1+3}$, the space $\mathcal{L}(\mathcal{O})$ is defined by additivity. Each $\mathcal{L}(\mathcal{O})$ is a subspace of $\mathcal{L}:=\omega^{-\frac{1}{2}} \widehat{\mathcal{D}_{\mathbb{R}}\left(\mathbb{R}^{3}\right)}+i \omega^{+\frac{1}{2}} \widehat{\mathcal{D}_{\mathbb{R}}\left(\mathbb{R}^{3}\right)}$, which is a real linear dense subspace of $\mathcal{K}$ invariant under the translations $U(t, \vec{x})$. Endowing $\mathcal{L}$ with the symplectic form

$$
\sigma\left(f_{1}, f_{2}\right):=-\operatorname{Im}\left\langle f_{1}, f_{2}\right\rangle,
$$

one obtains a net $\mathcal{O} \longmapsto \mathcal{L}(\mathcal{O}) \subset \mathcal{L}$ of symplectic spaces. This net fulfils covariance $(U(x) \mathcal{L}(\mathcal{O})=\mathcal{L}(\mathcal{O}+x))$, isotony $\left(\mathcal{L}\left(\mathcal{O}_{1}\right) \subset \mathcal{L}\left(\mathcal{O}_{2}\right)\right.$ if $\left.\mathcal{O}_{1} \subset \mathcal{O}_{2}\right)$ and symplectic locality $\left(\sigma\left(\mathcal{L}\left(\mathcal{O}_{1}\right), \mathcal{L}\left(\mathcal{O}_{2}\right)\right)=\{0\}\right.$ if $\left.\mathcal{O}_{1} \times \mathcal{O}_{2}\right)$

(Whereas covariance is almost trivial with the above definition, isotony follows from the elementary fact that $e^{i \omega t} \mathcal{L}(\mathcal{O}) \subset \mathcal{L}\left(\mathcal{O}+K_{|t|}\right)$, where $K_{r}:=\left\{\vec{x} \in \mathbb{R}^{3}|| \vec{x} \mid<r\right\}$, due to the support properties of the fundamental solution of the wave equation. Since the number of space dimensions is odd, the right-hand side can even be replaced with $\mathcal{L}\left(\mathcal{O}+\partial K_{|t|}\right)$, a fact referred to as Huygens' principle. Locality of the net $\mathcal{L}(\cdot)$, finally, is best seen by writing elements $f_{j} \in \mathcal{L}, j=1,2$ in the standard form $f_{j}=\omega^{-\frac{1}{2}} \hat{h}_{j}+i \omega^{+\frac{1}{2}} \hat{g}_{j}$ with $h_{j}, g_{j} \in \mathcal{D}_{\mathbb{R}}\left(\mathbb{R}^{3}\right)$. Then $\sigma\left(f_{1}, f_{2}\right)=\int d^{3} x\left(g_{1}(\vec{x}) h_{2}(\vec{x})-h_{1}(\vec{x}) g_{2}(\vec{x})\right)$, which vanishes if $\operatorname{supp} g_{j}, h_{j} \subset O_{j}$ for disjoint balls $O_{j} \subset \mathbb{R}^{3}$. Thus $\sigma\left(\mathcal{L}\left(\mathcal{O}_{1}\right), \mathcal{L}\left(\mathcal{O}_{2}\right)\right)=\{0\}$ in the special case when $\mathcal{O}_{j}=\left(\{0\} \times O_{j}\right)^{\prime \prime}$; this carries over to the general case $\mathcal{O}_{1} \times \mathcal{O}_{2}$ due to covariance, isotony and additivity.)

\footnotetext{
${ }^{1}$ By dropping for simplicity the indices 0 from $\mathcal{H}, U$ and (later) from $\mathfrak{A}$ and $\alpha_{x}$, we deviate from the convention set up in the Introduction.
} 
The net of observables is now given as

$$
\mathcal{O} \longmapsto \mathfrak{A}(\mathcal{O}):=\mathcal{W}(\mathcal{L}(\mathcal{O}))^{\prime \prime},
$$

where $\mathcal{O}$ runs through all bounded open subsets of $\mathbb{R}^{1+3}$. As a consequence of the corresponding properties of the net $\mathcal{L}$, it is isotonous, local and translation covariant with respect to $\alpha_{x}:=\operatorname{Ad} U(x)$. Since $\mathcal{L}$ is dense in $\mathcal{K}$, the quasilocal algebra $\mathfrak{A}$ of this net acts irreducibly on $\mathcal{H}$.

The charges under consideration are given in terms of automorphisms $\gamma \in$ AutA which are labeled uniquely by elements of the real vector space

$$
\mathcal{V}:=\omega^{-\frac{1}{2}} \widehat{\mathcal{D}_{\mathbb{R}}\left(\mathbb{R}^{3}\right)}+i \omega^{-\frac{3}{2}} \widehat{\mathcal{D}_{\mathbb{R}}\left(\mathbb{R}^{3}\right)} .
$$

Any element $\gamma \in \mathcal{V}$ gives rise to a linear form

$$
l_{\gamma}: \mathcal{L} \longrightarrow \mathbb{C}: f \longmapsto l_{\gamma}(f):=-\operatorname{Im} \int d^{3} k \overline{\gamma(\vec{k})} f(\vec{k})
$$

which is locally normal in the following sense: for each bounded region $\mathcal{O} \subset \mathbb{R}^{1+3}$, there is some vector $v_{\gamma}^{\mathcal{O}} \in \mathcal{K}$ such that

$$
l_{\gamma}(f)=-\operatorname{Im}\left\langle v_{\gamma}^{\mathcal{O}}, f\right\rangle \quad \text { for all } \quad f \in \mathcal{L}(\mathcal{O})
$$

(It is indeed not difficult to find such vectors $v_{\gamma}^{\mathcal{O}}$; a very elegant solution is the one from [34], where Huygens' principle is invoked for showing that $v_{\gamma}^{\mathcal{O}}:=\frac{1-e^{i \omega T}}{i \omega} i \omega \gamma$ (with $T=$ const $\left._{\gamma}+\operatorname{diam} \mathcal{O}\right)$ does the job. Notice that $i \omega \gamma \in \mathcal{L} \subset \mathcal{K}$ and that $\left\|\frac{1-e^{i \omega T}}{i \omega}\right\|=|T|$.) As a consequence, the automorphism $\gamma$ of $\mathcal{W}(\mathcal{L})$ defined by

$$
\gamma(W(f)):=e^{i l_{\gamma}(f)} W(f), \quad f \in \mathcal{L}
$$

is implemented on each algebra $\mathcal{W}(\mathcal{L}(\mathcal{O}))$ by the unitary $W\left(v_{\gamma}^{\mathcal{O}}\right)$ and therefore defines a unique automorphism $\gamma$ of the quasilocal algebra $\mathfrak{A}$ normal on each local von Neumann algebra $\mathfrak{A}(\mathcal{O})$.

There will be no risk of confusion in viewing the vector space $\mathcal{V}$ as an abelian subgroup of Aut $\mathfrak{A}$, and we therefore make no notational distinction between the two meanings of $\gamma$. A sum $\gamma_{1}+\gamma_{2}$ in $\mathcal{V}$ corresponds to the composition $\gamma_{1} \circ \gamma_{2}$ in AutA. Moreover, $\gamma_{1}$ and $\gamma_{2}$ define the same sector of $\mathfrak{A}$, i.e., they are unitarily equivalent in $\mathcal{B}(\mathcal{H})$, iff $\gamma_{1}-\gamma_{2} \in \mathcal{V} \cap \mathcal{K}$. In this case, the Weyl operator $W\left(\gamma_{1}-\gamma_{2}\right)$ is well defined and implements the unitary equivalence $\gamma_{1} \cong \gamma_{2}$ between the automorphisms of $\mathfrak{A}$.

Any $\gamma \in \mathcal{V}$ can be written uniquely in the standard form $\gamma=\omega^{-\frac{1}{2}} \hat{\sigma}+i \omega^{-\frac{3}{2}} \hat{\rho}$ with functions $\sigma, \rho \in \mathcal{D}_{\mathbb{R}}\left(\mathbb{R}^{3}\right)$. Since $\hat{\sigma}$ and $\hat{\rho}$ are test functions, it is obvious that $\gamma$ is square integrable, i.e., $\gamma \in \mathcal{K}$, iff $\hat{\rho}(0)=0$. As a consequence, the equivalence classes of charges are labeled by a single real parameter

$$
q_{\gamma}:=\hat{\rho}(0)=\int d^{3} x \rho(\vec{x})
$$

interpreted as the charge of the sector $[\gamma]$.

The unitary action $U(x)$ of the spacetime translations on $\mathcal{K}$ extends in the obvious way to an action on $\mathcal{V}$; the element $U(x) \gamma$ of $\mathcal{V}$ corresponds to the automorphism $\alpha_{x} \circ \gamma \circ \alpha_{x}^{-1}$ 
of $\mathfrak{A}$, and it is consistent to denote with $\gamma \longmapsto \gamma_{x}$ both actions of the translation by $x$. It is easily seen that $q_{\gamma_{x}}=q_{\gamma}$ and that the sectors are translation covariant. As a matter of fact, they even have positive energy [34].

Remark: The vector space $\mathcal{V}$ can be given a local structure analogous to the one of $\mathcal{L}$. A generic element $\gamma \in \mathcal{V}\left((\{0\} \times O)^{\prime \prime}\right)$ then reads $\gamma=\omega^{-\frac{1}{2}} \hat{\sigma}+i \omega^{-\frac{3}{2}} \hat{\rho}$ with $\operatorname{supp} \sigma, \rho \subset O$. Now if one evaluates the linear form $l_{\gamma}$ on $f=\omega^{-\frac{1}{2}} \hat{h}+i \omega^{+\frac{1}{2}} \hat{g}$, one gets

$$
l_{\gamma}(f)=\int d^{3} k\left(\frac{1}{\omega^{2}} \overline{\hat{\rho}(\vec{k})} \hat{h}(\vec{k})-\overline{\hat{\sigma}(\vec{k})} \hat{g}(\vec{k})\right)=\int d^{3} x\left(\Phi_{\rho}(\vec{x}) h(\vec{x})-\sigma(\vec{x}) g(\vec{x})\right),
$$

where $\Phi_{\rho}(\vec{x}):=\frac{1}{4 \pi} \int d^{3} y \frac{\rho(\vec{y})}{|\vec{x}-\vec{y}|}$ is the Coulomb potential of the charge distribution $\rho$. Apart from very special cases, the support of $\Phi_{\rho}$ is all of $\mathbb{R}^{3}$ and $l_{\gamma}$ does not vanish identically on any of the subspaces $\mathcal{L}(\mathcal{O})$. Viewed as an automorphism of $\mathfrak{A}, \gamma$ is thus localised everywhere. The observation that $(\{0\} \times O)^{\prime \prime}$ can nevertheless be regarded as the localisation region ("charge support") of $\gamma$ in a suitable sense is the starting point of [34].

\subsection{Poor Localisation in Front of the Vacuum}

It has been shown in [34] that the automorphisms $\gamma \in \mathcal{V}$ are not localisable in double cones in the sense of superselection theory. In Prop. 5.1 we will strengthen this result and show with closely related methods that their behaviour is much worse: they are not even localisable in causal complements of spacelike cones. Notice that this is a stronger statement than saying that the sectors are not localisable in spacelike cones.

Proposition 5.1 Let $C \subset \mathbb{R}^{3}$ be a pointed open convex cone having 0 as its apex and denote with $\mathcal{C}:=(\{0\} \times C)^{\prime \prime}$ its causal completion. Then, for any $\gamma \in \mathcal{V}$,

$$
\left.\left.\gamma\right|_{\mathfrak{A}(\mathcal{C})} \cong \mathrm{id}\right|_{\mathfrak{A}(\mathcal{C})} \quad \text { iff } \quad q_{\gamma}=0 .
$$

In view of the remarks in Section 5.1, the "if" part of this proposition is trivial: $q_{\gamma}=0$ implies $\gamma \in \mathcal{K}$, hence the unitary $W(\gamma)$ implements $\gamma$ on the whole algebra $\mathfrak{A}$. For the "only if" part, it has to be shown that $q_{\gamma} \neq 0$ entails that $\gamma$ and id $_{\mathfrak{A}}$ are inequivalent in restriction to the subalgebra $\mathfrak{A}(\mathcal{C})$. The standard method for proving such a statement is to exhibit a sequence of elements in $\mathfrak{A}(\mathcal{C})$ which converges weakly to different scalar multiples of the unit operator in the two representations. In the present case, this is facilitated by the fact that $\mathcal{C}$ is a cone. In order to take advantage of this, we first recall some facts about the dilation covariance of the model.

The dilation group $\mathbb{R}_{>0}$ acts unitarily on $\mathcal{K}$ and leaves the space $\mathcal{L}$ invariant. More precisely, $f \in \mathcal{L}(\mathcal{O})$ is mapped onto $f_{\lambda} \in \mathcal{L}(\lambda \mathcal{O})$, where $f_{\lambda}(\vec{k}):=\lambda^{\frac{3}{2}} f(\lambda \vec{k})$. Writing $f$ as $f=\omega^{-\frac{1}{2}} \hat{h}+i \omega^{+\frac{1}{2}} \hat{g}$, it is verified by a straightforward computation that this entails for the linear form $l_{\gamma}, \gamma \in \mathcal{V}$ :

$$
l_{\gamma}\left(f_{\lambda}\right)=\int \frac{d^{3} k}{\omega^{2}} \overline{\hat{\rho}(\vec{k} / \lambda)} \hat{h}(\vec{k})-\frac{1}{\lambda} \int d^{3} k \overline{\hat{\sigma}(\vec{k} / \lambda)} \hat{g}(\vec{k}) .
$$

In the limit $\lambda \rightarrow \infty, \vec{k} \longmapsto \overline{\hat{\rho}(\vec{k} / \lambda)} \hat{h}(\vec{k})$ converges to $\hat{\rho}(0) \hat{h}$ in the space of test functions, and since $\frac{2 \pi^{2}}{r}$ is the Fourier transform of $\frac{1}{\omega^{2}}$ in the sense of distributions, one obtains

$$
\lim _{\lambda \rightarrow \infty} l_{\gamma}\left(f_{\lambda}\right)=q_{\gamma} \kappa_{f} \quad \text { with } \quad \kappa_{f}:=2 \pi^{2} \int \frac{d^{3} x}{|\vec{x}|} h(\vec{x}) .
$$


This is used for proving the following lemma which will then allow us to complete the proof of Prop. 5.1.

Lemma 5.2 Let $f \in \mathcal{L}\left(\mathcal{O}^{\prime}\right)$, where $\mathcal{O} \subset \mathbb{R}^{1+3}$ is a neighbourhood of 0 . For any $\gamma \in \mathcal{V}$, one then has

$$
\underset{\lambda \rightarrow \infty}{\mathrm{w}-\lim _{i}} \gamma\left(W\left(f_{\lambda}\right)\right)=e^{i q_{\gamma} \kappa_{f}} e^{-\frac{1}{4}\|f\|^{2}} \mathbf{1} .
$$

Proof: Since the dilations act geometrically, it follows by locality from the special form of the localisation region of $f$ that $\lim _{\lambda \rightarrow \infty} \sigma\left(f_{\lambda}, f^{\prime}\right)=0$ for any $f^{\prime} \in \mathcal{L}$. Hence $\left(W\left(f_{\lambda}\right)\right)_{\lambda>0}$ is a central sequence of unitaries in $\mathcal{W}(\mathcal{L})$ whose set of weak limits is, by the irreducibility of the vacuum representation, a (nonempty) subset of $\mathbb{C} 1$. On the other hand, unitarity of the dilations permits us to evaluate this limit in the vacuum state: $\omega_{0}\left(W\left(f_{\lambda}\right)\right) \stackrel{\lambda \rightarrow \infty}{\longrightarrow} e^{-\frac{1}{4}\|f\|^{2}}$. But this means that $W\left(f_{\lambda}\right)$ has $e^{-\frac{1}{4}\|f\|^{2}} \mathbf{1}$ as its unique weak limit for $\lambda \rightarrow \infty$. From this and from equation (5.2) it now follows that $\gamma\left(W\left(f_{\lambda}\right)\right)=e^{i l_{\gamma}\left(f_{\lambda}\right)} W\left(f_{\lambda}\right)$ converges weakly to $e^{i q_{\gamma} \kappa_{f}} e^{-\frac{1}{4}\|f\|^{2}} \mathbf{1}$, as claimed.

Proof of Prop. 5.1: Let $\gamma \in \mathcal{V}$ with $q_{\gamma} \neq 0$ be given. Choose a nonvanishing, nonnegative test function $h \in \mathcal{D}_{\mathbb{R}}(C)$. Letting $f:=\omega^{-\frac{1}{2}} \hat{h}$, this implies $\kappa_{f} \neq 0$ and $f \in \mathcal{L}\left(\mathcal{C} \cap \mathcal{O}^{\prime}\right)$ for some neighbourhood $\mathcal{O} \subset \mathbb{R}^{1+3}$ of 0 . Since $e^{i q_{\gamma} \kappa_{f}} \neq 1$ can always be obtained by rescaling $h$, Lemma 5.2 shows that the weak limits (as $\lambda \rightarrow \infty)$ of $W\left(f_{\lambda}\right)$ and $\gamma\left(W\left(f_{\lambda}\right)\right)$ are different scalar multiples of the unit operator. But since $W\left(f_{\lambda}\right) \in \mathfrak{A}(\mathcal{C})$ for all $\lambda>0$, this implies $\left.\left.\gamma\right|_{\mathfrak{A}(\mathcal{C})} \nRightarrow \mathrm{id}\right|_{\mathfrak{A}(\mathcal{C})}$.

The physical interpretation of the family $\left(W\left(f_{\lambda}\right)\right)_{\lambda \rightarrow \infty}$ is that of a measurement of the asymptotic behaviour (in the spatial directions determined by the smearing function $h$ ) of the Coulomb potential $\Phi_{\rho}$ of the charge density $\rho$. In QED, one expects that operators measuring the asymptotic electric flux distribution play a similar role, cf. [6]. In the present model, the leading $1 / r$ part of the Coulomb potential is isotropic in all sectors $[\gamma]$. Mathematically, this is reflected by the factorising of the $\operatorname{limit} \lim l_{\gamma}\left(f_{\lambda}\right)$ as seen in equation (5.2).

Remark: With quite similar methods and based on the same physical picture, Prop. 5.1 can be strengthened still a bit further: if $q_{\gamma} \neq 0$, then $\pi_{0} \circ \gamma$ and $\pi_{0}$ are inequivalent in restriction to an algebra $\mathfrak{A}(\mathcal{N})$, where $\mathcal{N}:=\bigcup_{\lambda>0}(\mathcal{O}+(0, \lambda \vec{e}))($ with $\vec{e} \neq 0)$ is a uniform neighbourhood of a half-line.

\subsection{Infravacuum Background States}

In this section we introduce a class of background states in front of which the automorphisms $\gamma$ will be shown (in Section 5.4) to have better localisation properties. Apart from two modifications necessitated by the present model, these background states are of the same type as those introduced by Kraus, Polley and Reents [5] for describing infrared clouds in QED or, more generally, in any theory containing massless particles.

\subsubsection{Preliminaries on quasifree states}

First, we recall that a quasifree state on $\mathfrak{A}$ is a locally normal state $\omega_{T}$ of the form

$$
\omega_{T}(W(f))=e^{-\frac{1}{4}\|T f\|^{2}} \quad \text { for all } \quad f \in \mathcal{L} .
$$


Here $T: D_{T} \longrightarrow \mathcal{K}$ is a real linear, symplectic (i.e., fulfilling $\operatorname{Im}\langle T v, T w\rangle=\operatorname{Im}\langle v, w\rangle$, $\left.v, w \in D_{T}\right)$ operator defined on a dense, real linear subspace $D_{T} \supset \mathcal{L}$. In the case at hand, we will have in addition $\overline{T \mathcal{L}}=\mathcal{K}$ (see Lemma 5.5), which entails that $\omega_{T}$ is a pure state. Its GNS representation $\pi_{T}$ is irreducible; it can be taken to act on the vacuum Hilbert space $\mathcal{H}$, in which case it reads $\pi_{T}(W(f))=W(T f), f \in \mathcal{L}$.

Next, we express the real linear operator $T$ in terms of a pair of complex linear operators $T_{1}, T_{2}$ defined on complex linear subspaces $D_{T_{j}}$ of $\mathcal{K}$.

Lemma 5.3 Let $\Gamma: \mathcal{K} \longrightarrow \mathcal{K}$ be an antiunitary involution. Then the formulae

$$
\begin{aligned}
T & :=T_{2} \frac{1+\Gamma}{2}+T_{1} \frac{1-\Gamma}{2} \\
D_{T} & :=\left\{v \in \mathcal{K} \mid \frac{1+\Gamma}{2} v \in D_{T_{2}}, \frac{1-\Gamma}{2} v \in D_{T_{1}}\right\}
\end{aligned}
$$

establish a bijection between

- densely defined, $\Gamma$-invariant $\square^{2} \mathbb{R}$-linear operators $T: D_{T} \longrightarrow \mathcal{K}$ and

- densely defined, $\Gamma$-invariant $\mathbb{C}$-linear operators $T_{j}: D_{T_{j}} \longrightarrow \mathcal{K}, j=1,2$.

Moreover, $T$ is symplectic iff $\left\langle T_{1} u_{1}, T_{2} u_{2}\right\rangle=\left\langle u_{1}, u_{2}\right\rangle$ for all $u_{j} \in D_{T_{j}}$.

Since all these assertions can be checked by simple computations, we omit the formal proof of this lemma and merely point out that the converse formulae expressing $T_{1}$ and $T_{2}$ in terms of $T$ read

$$
\begin{array}{ll}
D_{T_{2}}=\left\{v \in \mathcal{K} \mid \frac{1+\Gamma}{2} \mathbb{C} v \subset D_{T}\right\}, & T_{2}=T \frac{1+\Gamma}{2}-i T \frac{1+\Gamma}{2} i, \\
D_{T_{1}}=\left\{v \in \mathcal{K} \mid \frac{1-\Gamma}{2} \mathbb{C} v \subset D_{T}\right\}, & T_{1}=T \frac{1-\Gamma}{2}+i T \frac{1-\Gamma}{2} i .
\end{array}
$$

Remark: The involution $\Gamma$ induces the notion of real and imaginary parts of vectors $v \in \mathcal{K}: \operatorname{Re} v=\frac{1+\Gamma}{2} v, \operatorname{Im} v=\frac{1-\Gamma}{2 i} v$. Then $T_{2}$ acts on the real and $T_{1}$ on the imaginary parts:

$$
\operatorname{Re} T v=T_{2} \operatorname{Re} v, \quad \operatorname{Im} T v=T_{1} \operatorname{Im} v, \quad v \in D_{T} .
$$

From now on, we fix $\Gamma$ to be pointwise complex conjugation in position space. In terms of momentum space wave functions $v \in \mathcal{K}$, this means

$$
(\Gamma v)(\vec{k}):=\overline{v(-\vec{k})} \text {. }
$$

For the sake of completeness, we point out that Kraus et al. used pointwise conjugation in momentum space for defining their background states in [5]. In their case as well as in ours, the choice of the involution $\Gamma$ is dictated by the set of sectors under consideration.

\subsubsection{Quasifree states with positive energy}

Before describing in detail the operators $T_{1}, T_{2}$, we introduce some notation: For any $\epsilon>0$, let $P_{\epsilon}: \mathcal{K} \longrightarrow \mathcal{K}$ be the projector onto the subspace $P_{\epsilon} \mathcal{K}=\{v \in \mathcal{K} \mid v(\vec{k})=0$ if $|\vec{k}|<\epsilon\}$ and denote with

$$
D_{0}:=\bigcup_{\epsilon>0} P_{\epsilon} \mathcal{K}
$$

\footnotetext{
${ }^{2}$ Here, $T: D_{T} \longrightarrow \mathcal{K}$ being $\Gamma$-invariant means $\Gamma D_{T}=D_{T}$ and $[\Gamma, T]=0$ on $D_{T}$.
} 
the dense subspace of functions vanishing in some neighbourhood of $\vec{k}=0$. Note that $\left[P_{\epsilon}, \Gamma\right]=0$ and $\Gamma D_{0}=D_{0}$. The subspace $D_{0}$ serves as a provisional domain for the operators $T_{1}$ and $T_{2}$.

Following [5] we now choose

- a sequence $\left(\epsilon_{i}\right)_{i \in \mathbb{N}}$ in $\mathbb{R}_{>0}$ satisfying $\epsilon_{i+1}<\epsilon_{i}$ and $\epsilon_{i} \stackrel{i \rightarrow \infty}{\longrightarrow} 0$.

This sequence induces a decomposition of momentum space into concentric spherical shells. The projections onto the associated spectral subspaces of $\mathcal{K}$ will be denoted by $P_{i}:=P_{\epsilon_{i+1}}-P_{\epsilon_{i}}$. For notational convenience, we also put $P_{0}:=P_{\epsilon_{1}}$.

- a sequence $\left(Q_{i}\right)_{i \in \mathbb{N}}$ of orthogonal projections in $\mathcal{K}$ with finite rank $\operatorname{rk} Q_{i}$ satisfying $Q_{i} \Gamma=\Gamma Q_{i}$ and $Q_{i} P_{i}=Q_{i}$.

- a sequence $\left(b_{i}\right)_{i \in \mathbb{N}}$ in ]0,1[ satisfying $b_{i} \stackrel{i \rightarrow \infty}{\longrightarrow} 0$ and $\sum_{i} \frac{\epsilon_{i}}{b_{i}^{2}} \operatorname{rk} Q_{i}<\infty$.

If, e.g., the $\epsilon_{i}$ decrease exponentially and $\operatorname{rk} Q_{i}$ is polynomially bounded, this can be satisfied by $b_{i} \propto i^{-\alpha}, \alpha>0$.

With these data, define $\mathbb{C}$-linear operators $T_{1}, T_{2}$ on the subspace $D_{0}$ by

$$
T_{1}:=\mathbf{1}+\operatorname{s-lim}_{n \rightarrow \infty} \sum_{i=1}^{n}\left(b_{i}-1\right) Q_{i}, \quad T_{2}:=\mathbf{1}+\underset{n \rightarrow \infty}{\operatorname{s}-\lim _{i=1}} \sum_{i=1}^{n}\left(\frac{1}{b_{i}}-1\right) Q_{i} .
$$

Since on every $v \in D_{0}$ the number of terms which contribute on the right-hand side is finite, these operators are well defined and map $D_{0}$ into itself. Moreover, the relations

$$
T_{1} P_{i}=\left(\left(\mathbf{1}-Q_{i}\right)+b_{i} Q_{i}\right) P_{i}, \quad T_{2} P_{i}=\left(\left(\mathbf{1}-Q_{i}\right)+\frac{1}{b_{i}} Q_{i}\right) P_{i}
$$

show that the subspace $P_{i} \mathcal{K}$ decomposes into a subspace $\left(\mathbf{1}-Q_{i}\right) P_{i} \mathcal{K}$ where both $T_{1}$ and $T_{2}$ act trivially and an orthogonal (finite dimensional) subspace $Q_{i} P_{i} \mathcal{K}=Q_{i} \mathcal{K}$ where they act as multiplications with the scalars $b_{i}$ and $\frac{1}{b_{i}}$, respectively. As a consequence, $T_{1}$ and $T_{2}$ are inverses of each other. Because of $\lim _{i \rightarrow \infty} b_{i}=0, T_{1}$ is bounded (with norm 1 ), whereas $T_{2}$ is not. Also, it is clear that $T_{1}$ and $T_{2}$ are $\Gamma$-invariant and symmetric. It follows in particular that $\left\langle T_{1} u_{1}, T_{2} u_{2}\right\rangle=\left\langle u_{1}, T_{1} T_{2} u_{2}\right\rangle=\left\langle u_{1}, u_{2}\right\rangle$ for any $u_{1}, u_{2} \in D_{0}$. We are thus in the situation of Lemma 5.3 and obtain an unbounded symplectic operator

$$
T: D_{0} \longrightarrow \mathcal{K}, \quad T=T_{2} \frac{1+\Gamma}{2}+T_{1} \frac{1-\Gamma}{2} .
$$

In the next step, $T$ has to be extended to a larger domain $D_{T} \supset \mathcal{L}$. To this end, we analyse its singular behaviour for $|\vec{k}| \rightarrow 0$ by comparing it with powers of (a regularised version $\omega_{\mathrm{r}}$ of) the one-particle energy $\omega$. Setting

$$
\omega_{\mathrm{r}}:=\omega\left(\mathbf{1}-P_{0}\right)+\epsilon_{1} P_{0}= \begin{cases}\omega & \text { on }\left(\mathbf{1}-P_{0}\right) \mathcal{K} \\ \epsilon_{1} \mathbf{1} & \text { on } P_{0} \mathcal{K}\end{cases}
$$

and noting that $\omega_{\mathrm{r}}^{1 / 2} D_{0}=D_{0}$, we have:

Lemma 5.4 The operator $T_{2} \omega_{\mathrm{r}}^{1 / 2}$ is bounded. 
Proof: Making use of $\left\|\omega_{\mathrm{r}} P_{i}\right\|=\epsilon_{i}$ for $i \in \mathbb{N}$, one gets for $v \in D_{0}$ the estimate

$$
\begin{aligned}
\left\|\left(T_{2}-\mathbf{1}\right) \omega_{\mathrm{r}}^{\frac{1}{2}} v\right\|^{2} & =\left\|\sum_{i}\left(\frac{1}{b_{i}}-1\right) Q_{i} \omega_{\mathrm{r}}^{\frac{1}{2}} v\right\|^{2}=\sum_{i}\left(\frac{1}{b_{i}}-1\right)^{2}\left\langle\omega_{\mathrm{r}}^{\frac{1}{2}} v, Q_{i} \omega_{\mathrm{r}}^{\frac{1}{2}} v\right\rangle \\
& \leq \sum_{i}\left(\frac{1}{b_{i}}-1\right)^{2}\left\|Q_{i}\right\|\left\langle\omega_{\mathrm{r}}^{\frac{1}{2}} v, P_{i} \omega_{\mathrm{r}}^{\frac{1}{2}} v\right\rangle \leq \sum_{i}\left(\frac{1}{b_{i}}-1\right)^{2} \mathrm{rk} Q_{i} \epsilon_{i}\|v\|^{2} .
\end{aligned}
$$

From the conditions imposed on the $b_{i}$ it follows that $\sum_{i}\left(\frac{1}{b_{i}}-1\right)^{2} \operatorname{rk} Q_{i} \epsilon_{i}$ is finite. Thus $\left(T_{2}-\mathbf{1}\right) \omega_{\mathrm{r}}^{1 / 2}$ is bounded, hence also $T_{2} \omega_{\mathrm{r}}^{1 / 2}$.

One can now extend $T_{1}$ by continuity to all of $\mathcal{K}=: D_{T_{1}}$ and $T_{2}$ by the formula

$$
T_{2} v:=T_{2} \omega_{\mathrm{r}}^{\frac{1}{2}} \omega_{\mathrm{r}}^{-\frac{1}{2}} v, \quad v \in \omega_{\mathrm{r}}^{\frac{1}{2}} \mathcal{K}
$$

to the dense subspace $\omega_{\mathrm{r}}^{\frac{1}{2}} \mathcal{K}=: D_{T_{2}}$. (Strictly speaking, the symbol $T_{2} \omega_{\mathrm{r}}^{\frac{1}{2}}$ on the right-hand side stands for the continuous extension to $\mathcal{K}$ of the operator considered in Lemma 5.4.) Notice that $T_{1}$ and $T_{2}$ still are $\Gamma$-invariant. The relevant properties of the resulting symplectic operator $T$ are collected in the next lemma:

\section{Lemma 5.5}

i. $D_{T}:=\left\{v \in \mathcal{K} \mid \frac{1+\Gamma}{2} v \in \omega_{\mathrm{r}}^{1 / 2} \mathcal{K}\right\}$ is a real linear dense subspace of $\mathcal{K}$.

ii. $T=T_{2} \frac{1+\Gamma}{2}+T_{1} \frac{1-\Gamma}{2}$ is well defined on $D_{T}$.

iii. $T: D_{T} \longrightarrow \mathcal{K}$ is a symplectic operator.

iv. $\mathcal{L} \subset D_{T}$, and $T \mathcal{L}$ is dense in $\mathcal{K}$.

Proof: Part i is obvious, since $D_{0} \subset D_{T}$; Part ii has been shown in the previous paragraph. For iii, we have to show that $\left\langle T_{1} u_{1}, T_{2} u_{2}\right\rangle=\left\langle u_{1}, u_{2}\right\rangle$ remains true for all $u_{1} \in D_{T_{1}}$ and $u_{2} \in D_{T_{2}}$. First, assume $u_{1} \in D_{0}$. Since $D_{0}$ is dense in $\mathcal{K}$ and invariant under $\omega_{\mathrm{r}}^{1 / 2}$, there exists a sequence $u_{2}^{(n)} \in D_{0}, n \in \mathbb{N}$ such that $\omega_{\mathrm{r}}^{-\frac{1}{2}} u_{2}=\lim \omega_{\mathrm{r}}^{-\frac{1}{2}} u_{2}^{(n)}$, implying $u_{2}=\lim u_{2}^{(n)}$. Using the boundedness of $T_{2} \omega_{\mathrm{r}}^{1 / 2}$, we can compute

$$
\begin{aligned}
\left\langle T_{1} u_{1}, T_{2} u_{2}\right\rangle & =\left\langle T_{1} u_{1}, T_{2} \omega_{\mathrm{r}}^{\frac{1}{2}} \omega_{\mathrm{r}}^{-\frac{1}{2}} u_{2}\right\rangle=\left\langle T_{1} u_{1}, T_{2} \omega_{\mathrm{r}}^{\frac{1}{2}} \lim _{n \rightarrow \infty} \omega_{\mathrm{r}}^{-\frac{1}{2}} u_{2}^{(n)}\right\rangle \\
& =\lim _{n \rightarrow \infty}\left\langle T_{1} u_{1}, T_{2} \omega_{\mathrm{r}}^{\frac{1}{2}} \omega_{\mathrm{r}}^{-\frac{1}{2}} u_{2}^{(n)}\right\rangle=\lim _{n \rightarrow \infty}\left\langle u_{1}, u_{2}^{(n)}\right\rangle=\left\langle u_{1}, u_{2}\right\rangle .
\end{aligned}
$$

Since $T_{1}$ is bounded, the restriction on $u_{1}$ can now be dropped by continuity, thus yielding the assertion. Finally, $\mathcal{L} \subset D_{T}$ is obvious, and the remaining part of iv is equivalent, in terms of $T_{1}$ and $T_{2}$, to

$$
\begin{array}{clc}
\frac{1+\Gamma}{2} T \mathcal{L}=T_{2} \frac{1+\Gamma}{2} \mathcal{L}=T_{2} \omega^{-\frac{1}{2}} \widehat{\mathcal{D}_{\mathbb{R}}\left(\mathbb{R}^{3}\right)} & \text { is dense in } & \frac{1+\Gamma}{2} \mathcal{K}, \\
\frac{1-\Gamma}{2 i} T \mathcal{L}=T_{1} \frac{1-\Gamma}{2 i} \mathcal{L}=T_{1} \omega^{\frac{1}{2}} \widehat{\mathcal{D}_{\mathbb{R}}\left(\mathbb{R}^{3}\right)} & \text { is dense in } & \frac{1-\Gamma}{2 i} \mathcal{K} .
\end{array}
$$

By $\mathbb{C}$-linearity, this in turn is equivalent to $T_{2} \omega^{-\frac{1}{2}} \widehat{\mathcal{D}_{\mathbb{C}}\left(\mathbb{R}^{3}\right)}=T_{2} \omega_{\mathrm{r}}^{\frac{1}{2}} \omega_{\mathrm{r}}^{-\frac{1}{2}} \omega^{-\frac{1}{2}} \widehat{\mathcal{D}_{\mathbb{C}}\left(\mathbb{R}^{3}\right)}$ and $T_{1} \omega^{\frac{1}{2}} \widehat{\mathcal{D}_{\mathbb{C}}\left(\mathbb{R}^{3}\right)}$ both being dense in $\mathcal{K}$. But this is implied by the fact that, on the one hand, both operators $T_{2} \omega_{\mathrm{r}}^{1 / 2}$ and $T_{1}$ are bounded and have dense images (since they are 
invertible on the dense, invariant subspace $\left.D_{0}\right)$ and that, on the other hand, the subspaces $\omega_{\mathrm{r}}^{-\frac{1}{2}} \omega^{-\frac{1}{2}} \widehat{\mathcal{D}_{\mathbb{C}}\left(\mathbb{R}^{3}\right)}$ and $\omega^{\frac{1}{2}} \widehat{\mathcal{D}_{\mathbb{C}}\left(\mathbb{R}^{3}\right)}$ are dense in $\mathcal{K}$ (by the spectral calculus of $\omega$ ).

With the above preparations, we can define a state $\omega_{T}: \mathfrak{A} \longrightarrow \mathbb{C}$ and analyse its main properties.

Proposition 5.6 The quasifree state $\omega_{T}$, defined on $\mathcal{W}(\mathcal{L})$ by

$$
\omega_{T}(W(f))=e^{-\frac{1}{4}\|T f\|^{2}}, \quad f \in \mathcal{L},
$$

extends to a unique locally normal state $\omega_{T}$ on the quasilocal algebra $\mathfrak{A}$. This state is pure and has positive energy.

Proof: The difficult part of this proof is to obtain local normality of $\omega_{T}$ on the net $\mathcal{O} \longmapsto \mathcal{W}(\mathcal{L}(\mathcal{O}))$ of Weyl algebras. To this end, recall that $T$ is $($ on $\mathcal{L})$ the strong limit of symplectic operators $T_{n}$ such that $T_{n}-\mathbf{1}$ have finite rank. As a consequence, the associated quasifree states $\omega_{T_{n}}$ are vector states in the vacuum representation and converge weakly to $\omega_{T}$ on $\mathcal{W}(\mathcal{L})$. Now since the Fredenhagen-Hertel compactness condition $\mathrm{C}_{\sharp}[20,2 \mathbb{2}]$ is known to be fulfilled in the present model, one can conclude that $\omega_{T}$ is locally normal if the sequence $\left(\omega_{T_{n}}\right)_{n \in \mathbb{N}}$ is bounded with respect to some exponential energy norm $\|\cdot\|_{\beta}$, $\beta>0$. (These norms are defined by $\|\omega\|_{\beta}^{2}:=\omega\left(e^{2 \beta H}\right), H$ denoting the Hamiltonian on $\mathcal{H}$.) But this follows from $\sum_{i} \frac{\epsilon_{i}}{b_{i}^{2}} \operatorname{rk} Q_{i}<\infty$, as F. Hars has shown in [2:3], adapting ideas from [5]. (Although our involution $\Gamma$ differs from that of [5, [2.3], the arguments leading to this conclusion are still valid.) Thus $\omega_{T}$ is indeed locally normal on $\mathcal{W}(\mathcal{L})$ and therefore extends uniquely to a locally normal state on $\mathfrak{A}$. Since it is a weak limit of states in the vacuum representation with positive energy, the arguments of Buchholz and Doplicher [I.9] can be applied to show that $\omega_{T}$ has positive energy, too. Finally, the relation $\overline{T \mathcal{L}}=\mathcal{K}$, established in Lemma 5.5, implies that $\omega_{T}$ is pure, as has been noted at the very beginning of this section.

Remark: The inequality $\sum_{i} \frac{\epsilon_{i}}{b_{i}^{2}} \operatorname{rk} Q_{i}<\infty$, which played a crucial role in the preceding proof, has a direct physical interpretation. Indeed, performing the limit $\omega_{T_{n}} \rightarrow \omega_{T}$ corresponds to the excitation of more and more low-energy "photon" modes in comparison to the vacuum, namely those singled out by the projections $Q_{i}, i=1, \ldots, n$ which appear in $T$. Since $\frac{1}{b_{i}}$ measures the amplitude of these modes, each of them carries an energy of about $\frac{\epsilon_{i}}{b_{i}^{2}}$. Hence the modes in the energy interval $\left[\epsilon_{i+1}, \epsilon_{i}\right]$ contribute with (at most) $\frac{\epsilon_{i}}{b_{i}^{2}} \operatorname{rk} Q_{i}$ to the mean energy of the state $\omega_{T}$, and the above inequality thus means that $\omega_{T}$ describes an infrared cloud with finite total energy. We conjecture that these arguments can be sharpened in order to prove that the GNS representation $\pi_{T}$ of $\omega_{T}$ is an infravacuum representation in the sense of Section 2.2. We therefore also refer to the states $\omega_{T}$ of the present form as infravacuum states.

\subsubsection{KPR-like quasifree states}

We reach our goal of improving the localisation of the automorphisms $\gamma$ by considering a special class of infravacuum representations. The main idea, due to [5], is to control the angular momentum carried by the low-energy modes. It may be formalised as follows. 
Definition: The symplectic operator $T$ based on the sequences $\epsilon_{i}, Q_{i}, b_{i}$ as described above, the corresponding state $\omega_{T}$ and its GNS representation $\pi_{T}$ are called KPR-like if the following additional conditions are fulfilled:

1. The sequence $\left(\ln \frac{\epsilon_{i}}{\epsilon_{i+1}}\right)_{i \in \mathbb{N}}$ is polynomially bounded, and $\sum_{i} b_{i}^{2} \ln \frac{\epsilon_{i}}{\epsilon_{i+1}}<\infty$.

2. With respect to the tensor product structure of the subspaces $P_{i} \mathcal{K}$ given by $P_{i} \mathcal{K} \cong L^{2}\left(\left[\epsilon_{i+1}, \epsilon_{i}\right], \omega^{2} d \omega\right) \otimes L^{2}\left(S^{2}\right)$, the projections $Q_{i}$ read

$$
Q_{i}=\frac{\left|\xi_{i}\right\rangle\left\langle\xi_{i}\right|}{\left\langle\xi_{i} \mid \xi_{i}\right\rangle} \otimes \tilde{Q}_{i} \quad \text { with } \quad \tilde{Q}_{i}:=\sum_{0<\ell \leq i} \sum_{m=-\ell}^{\ell}\left|Y_{\ell m}\right\rangle\left\langle Y_{\ell m}\right| ;
$$

here the vector $\xi_{i} \in L^{2}\left(\left[\epsilon_{i+1}, \epsilon_{i}\right], \omega^{2} d \omega\right)$ is given by $\xi_{i}(\omega)=\omega^{-\frac{3}{2}}$ and $Y_{\ell m} \in L^{2}\left(S^{2}\right)$ are the spherical harmonics.

This definition has been formulated so as to imply the regularity property of the bounded operator $T_{1}$ formulated in Lemma 5.7. It is only through this result that the two additional properties of KPR-like infravacua enter the analysis of Section 5.4. It is apparent from the ensuing proof that the above definition may be generalised in several respects. However, we refrain from discussing these possibilities here.

In contrast, we draw the reader's attention to the following crucial difference between our KPR-like states and the "true" KPR states as defined in [5]: In our case, the projection $\tilde{Q}_{i}$ contains no summand $\left|Y_{00}\right\rangle\left\langle Y_{00}\right|$. In physical terms, this means that the infrared cloud does not contain any spherically symmetric low-energy modes. Such a restriction is necessary, since it is precisely by such modes or, equivalently, by the isotropic longrange behaviour of the Coulomb potential, that the sectors $[\gamma]$ differ from each other. Too strong an $\ell=0$ contribution to the infrared cloud would therefore render the sectors indistinguishable in front of that background (see the remarks at the end of Section 5.4). This seemingly artificial restriction on the background states mimics the situation in QED, where the Coulomb field $\overrightarrow{\mathcal{E}}(\vec{k}) \sim i \vec{k} / \omega^{2}$ cannot be compensated by transverse photons.

Lemma 5.7 Let the sequences $\epsilon_{i}, Q_{i}, b_{i}$ be such that $\omega_{T}$ is a KPR-like state. Let $u \in \mathcal{K}$ be a vector which has in a neighbourhood of $\vec{k}=0$ the form $u(\vec{k})=\eta(\vec{k} /|\vec{k}|)$, where $\eta \in C^{\infty}\left(S^{2}\right) \subset L^{2}\left(S^{2}\right)$. Then the sequence $\left(T_{1} \omega^{-\frac{3}{2}} P_{\epsilon_{n}} u\right)_{n \in \mathbb{N}}$ converges iff $\left\langle Y_{00}, \eta\right\rangle=0$.

Proof: Without any restriction, one may assume $u=c \otimes \eta$ with $c(\omega)=1$ if $\omega<\epsilon_{1}$. Then one has for $0<m<n$

$$
\begin{aligned}
T_{1} \omega^{-\frac{3}{2}} P_{\epsilon_{n}} u & -T_{1} \omega^{-\frac{3}{2}} P_{\epsilon_{m}} u=T_{1} \omega^{-\frac{3}{2}} \sum_{i=m}^{n-1} P_{i}(c \otimes \eta)=\sum_{i=m}^{n-1} T_{1} P_{i}\left(\xi_{i} \otimes \eta\right) \\
= & \sum_{i=m}^{n-1}\left(\left(\mathbf{1}-Q_{i}\right)+b_{i} Q_{i}\right)\left(\xi_{i} \otimes \eta\right)=\sum_{i=m}^{n-1} \xi_{i} \otimes\left(\left(\mathbf{1}-\tilde{Q}_{i}\right) \eta+b_{i} \tilde{Q}_{i} \eta\right) .
\end{aligned}
$$

To estimate the norm of this sum, one uses $\left\|\xi_{i}\right\|^{2}=\int_{\epsilon_{i+1}}^{\epsilon_{i}} \omega^{2} d \omega \frac{1}{\omega^{3}}=\ln \frac{\epsilon_{i}}{\epsilon_{i+1}}$ and obtains

$$
\left\|T_{1} \omega^{-\frac{3}{2}} P_{\epsilon_{n}} u-T_{1} \omega^{-\frac{3}{2}} P_{\epsilon_{m}} u\right\|^{2} \leq \sum_{i=m}^{n-1} \ln \frac{\epsilon_{i}}{\epsilon_{i+1}}\left(\left\|\left(\mathbf{1}-\tilde{Q}_{i}\right) \eta\right\|^{2}+b_{i}^{2}\|\eta\|^{2}\right) .
$$


Now assume that $\left\langle Y_{00}, \eta\right\rangle=0$. Then $\left\|\left(\mathbf{1}-\tilde{Q}_{i}\right) \eta\right\|^{2}=\sum_{\ell>i} \sum_{m}\left|\left\langle Y_{\ell m}, \eta\right\rangle\right|^{2}$, and since $\eta$ is smooth (and thus contained in the domain of any power of the angular momentum operator), one has for any $N \in \mathbb{N}$ some constant $c_{N}$ such that $\left\|\left(\mathbf{1}-\tilde{Q}_{i}\right) \eta\right\|^{2} \leq \frac{c_{N}}{i^{N}}$ for all $i \in \mathbb{N}$. Therefore

$$
\left\|T_{1} \omega^{-\frac{3}{2}} P_{\epsilon_{n}} u-T_{1} \omega^{-\frac{3}{2}} P_{\epsilon_{m}} u\right\|^{2} \leq \sum_{i=m}^{n-1} \ln \frac{\epsilon_{i}}{\epsilon_{i+1}}\left(\frac{c_{N}}{i^{N}}+b_{i}^{2}\|\eta\|^{2}\right) .
$$

With suitably chosen $N$, the right-hand side vanishes as $m, n \rightarrow \infty$ due to the conditions imposed on $\epsilon_{i}$ and $b_{i}$. Hence $\left(T_{1} \omega^{-\frac{3}{2}} P_{\epsilon_{n}} u\right)_{n \in \mathbb{N}}$ is a Cauchy sequence and thus convergent. Conversely, assume $\left\langle Y_{00}, \eta\right\rangle \neq 0$. Writing $\eta$ as $\eta=\left\langle Y_{00}, \eta\right\rangle Y_{00}+\eta_{1}$, the above argument shows that $\left(T_{1} \omega^{-\frac{3}{2}} P_{\epsilon_{n}}\left(c \otimes \eta_{1}\right)\right)_{n \in \mathbb{N}}$ is convergent. Hence $\left(T_{1} \omega^{-\frac{3}{2}} P_{\epsilon_{n}} u\right)_{n \in \mathbb{N}}$ is divergent because $\left(T_{1} \omega^{-\frac{3}{2}} P_{\epsilon_{n}}\left(c \otimes Y_{00}\right)\right)_{n \in \mathbb{N}}=\left(\omega^{-\frac{3}{2}} P_{\epsilon_{n}}\left(c \otimes Y_{00}\right)\right)_{n \in \mathbb{N}}$ is.

We end this section with a result which shows that the KPR-like infravacua do not affect the superselection structure of the present model. Like the previous lemma, it makes essential use of the absence of a $\left|Y_{00}\right\rangle\left\langle Y_{00}\right|$ term in $\tilde{Q}_{i}$, now reflected by the fact that $T f=f$ for all rotation invariant elements $f \in D_{T}$.

Proposition 5.8 Let $\pi_{T}$ be a KPR-like infravacuum representation. Then one has for any $\gamma_{1}, \gamma_{2} \in \mathcal{V}$

$$
\pi_{0} \circ \gamma_{1} \cong \pi_{0} \circ \gamma_{2} \quad \text { iff } \quad \pi_{T} \circ \gamma_{1} \cong \pi_{T} \circ \gamma_{2}
$$

Proof: Let $\pi_{0} \circ \gamma_{1} \cong \pi_{0} \circ \gamma_{2}$. Then $\gamma:=\gamma_{1}-\gamma_{2} \in \mathcal{V}$ has charge $q_{\gamma}=0$, as noted in Section 5.1, which does not only yield $\gamma \in \mathcal{K}$, but even $\gamma \in D_{T}$. Hence, the unitary $W(T \gamma)$ is well defined and intertwines the representations $\pi_{T} \circ \gamma_{1}$ and $\pi_{T} \circ \gamma_{2}$. Conversely, assume $\pi_{0} \circ \gamma_{1} \neq \pi_{0} \circ \gamma_{2}$, i.e., $q_{\gamma_{1}} \neq q_{\gamma_{2}}$. For any rotation invariant test function $h \in \mathcal{D}_{\mathbb{R}}\left(\mathbb{R}^{3} \backslash\{0\}\right)$, one has $\omega^{-\frac{1}{2}} \hat{h}=: f \in \mathcal{L}\left(\mathcal{O}^{\prime}\right)$ for some open neighbourhood $\mathcal{O} \subset \mathbb{R}^{1+3}$ of 0 and (if $f_{\lambda}$ is the image of $f$ under a dilation by $\left.\lambda \in \mathbb{R}_{>0}\right) T f_{\lambda}=f_{\lambda}$. Lemma 5.2 therefore implies $\pi_{T} \circ \gamma_{j}\left(W\left(f_{\lambda}\right)\right)=\gamma_{j}\left(W\left(f_{\lambda}\right)\right) \stackrel{\lambda \rightarrow \infty}{\longrightarrow} e^{i q_{\gamma_{j}} \kappa_{f}} e^{-\frac{1}{4}\|f\|^{2}}$ 1. As it is always possible to obtain $e^{i q_{\gamma_{1}} \kappa_{f}} \neq e^{i q_{\gamma_{2}} \kappa_{f}}$ by a suitable choice of $h$, the same argument as in the proof of Prop. 5.1 yields $\pi_{T} \circ \gamma_{1} \neq \pi_{T} \circ \gamma_{2}$.

\subsection{Better Localisation in Front of KPR-like Infravacua}

The main aim of this section is to prove Proposition 5.9 about the localisation of the sectors $[\gamma]$ in front of KPR-like infravacuum backgrounds. We then add some comments on variants of the localisation mechanism. In the sequel, $\mathcal{C}=(\{t\} \times C)^{\prime \prime}$ denotes an upright spacelike cone whose basis is the open convex cone $C \subset \mathbb{R}^{3}$ at time $t$.

Proposition 5.9 Let $\pi_{T}$ be a KPR-like infravacuum representation, and let $\gamma \in \mathcal{V}$. Then one has for any upright spacelike cone $\mathcal{C}$ :

$$
\left.\left.\pi_{T} \circ \gamma\right|_{\mathfrak{A}\left(\mathcal{C}^{\prime}\right)} \cong \pi_{T}\right|_{\mathfrak{A}\left(\mathcal{C}^{\prime}\right)}
$$


To prove this assertion, we will first deal with a special case in which the relevant computations can be carried out quite explicitly. The formal proof will eventually be completed by reducing the general case to this special one.

The case to be discussed first amounts to the following two assumptions:

- $\mathcal{C}=(\{0\} \times C)^{\prime \prime}$ and the apex of $C$ is the origin $0 \in \mathbb{R}^{3}$;

- $\gamma \in \mathcal{V}$ has the special form $\gamma=i \omega^{-\frac{3}{2}} \hat{\rho}$, where $\rho \in \mathcal{D}_{\mathbb{R}}\left(\mathbb{R}^{3}\right)$ satisfies $\rho=-\Delta \Phi$ with a rotation invariant function $\Phi \in C_{\mathbb{R}}^{\infty}\left(\mathbb{R}^{3}\right)$ obeying, for some $0<r_{1}<r_{2}<\infty$,

$$
\Phi(\vec{x})= \begin{cases}0 & \text { if }|\vec{x}|<r_{1} \\ \frac{q_{\gamma}}{4 \pi|\vec{x}|} & \text { if }|\vec{x}|>r_{2}\end{cases}
$$

To begin with, we note that the cone $C \subset \mathbb{R}^{3}$ determines, by projection onto the unit sphere $S^{2}$, a subset of $S^{2}$ which we denote by $C$, too. Then we choose a function $\chi^{C} \in C_{\mathbb{R}}^{\infty}\left(S^{2}\right)$ with the properties

$$
\text { (i) }\left.\chi^{C}\right|_{S^{2} \backslash C}=1 \quad \text { and } \quad \text { (ii) }\left\langle Y_{00}, \chi^{C}\right\rangle=0 \text {, }
$$

and denote with $\Phi^{C} \in C_{\mathbb{R}}^{\infty}\left(\mathbb{R}^{3}\right)$ the product ${ }^{\beta}$

$$
\Phi^{C}(\vec{x}):=\left(\Phi \cdot \chi^{C}\right)(\vec{x}):=\Phi(\vec{x}) \chi^{C}\left(\frac{\vec{x}}{|\vec{x}|}\right) .
$$

This function will now be used to construct a vector $v_{T}^{C} \in \mathcal{K}$ such that the unitary $W\left(v_{T}^{C}\right)$ intertwines from $\pi_{T}$ to $\pi_{T} \circ \gamma$ on the $\mathrm{C}^{*}$-algebra $\mathcal{W}\left(\mathcal{L}\left(\mathcal{C}^{\prime}\right)\right)$. Notice that if there were a vector $v_{\gamma}^{C} \in D_{T}$ such that $-\operatorname{Im}\left\langle v_{\gamma}^{C}, \cdot\right\rangle=l_{\gamma}(\cdot)$ on $\mathcal{L}\left(\mathcal{C}^{\prime}\right)$, then $T v_{\gamma}^{C}$ could be taken for $v_{T}^{C}$. Such a $v_{\gamma}^{C}$ of course does not exist (unless $q_{\gamma}=0$ ), but there exists a sequence $v_{n}^{C}$ of vectors in $D_{0}$ approximating it. To obtain this sequence, we notice that the function

$$
-\Delta \Phi^{C}=\rho \cdot \chi^{C}+\frac{\Phi}{r^{2}} \cdot \vec{L}^{2} \chi^{C}
$$

is square-integrable. $\left(\vec{L}^{2}: \mathcal{C}_{\mathbb{R}}^{\infty}\left(S^{2}\right) \longrightarrow \mathcal{C}_{\mathbb{R}}^{\infty}\left(S^{2}\right)\right.$ is the square of the angular momentum operator.) Hence its Fourier transform $u^{C}:=-\widehat{\Delta \Phi^{C}}$ is in $\mathcal{K}$, and

$$
v_{n}^{C}:=i \omega^{-\frac{3}{2}} P_{\epsilon_{n}} u^{C}, \quad n \in \mathbb{N}
$$

is the above-mentioned sequence. Before checking that (due to property (i) of $\chi^{C}$ ) it really does approximate $l_{\gamma}$ on $\mathcal{L}\left(\mathcal{C}^{\prime}\right)$, we observe how property (ii) affects the behaviour of $u^{C}$ in a neighbourhood of the origin.

Lemma 5.10 There exists a smooth function $\eta \in C^{\infty}\left(S^{2}\right)$ with $\left\langle Y_{00}, \eta\right\rangle=0$ and an analytic function $R: \mathbb{R}^{3} \longrightarrow \mathbb{C}$ with $R(0)=0$ such that

$$
u^{C}(\vec{k})=\eta\left(\frac{\vec{k}}{|\vec{k}|}\right)+R(\vec{k}) \quad \text { for } \quad \vec{k} \neq 0
$$

\footnotetext{
${ }^{3}$ We use the notation $\Psi \cdot \eta$ for the pointwise product of a rotation invariant function $\Psi$ and the function $\vec{u} \longmapsto \eta\left(\frac{\vec{u}}{|\vec{u}|}\right)$, where $\eta \in C^{\infty}\left(S^{2}\right)$. For definiteness, we let $(\Psi \cdot \eta)(0):=0$.
} 
Proof: Let $\mathcal{S}_{00}$ denote the set of all rotation invariant test functions. Since $\left\langle Y_{00}, \vec{L}^{2} \chi^{C}\right\rangle=0$, there exists a unique distribution $F_{1}$ on $\mathbb{R}^{3}$ which is homogeneous of degree -3 , coincides on $\mathbb{R}^{3} \backslash\{0\}$ with $\frac{q_{\gamma}}{4 \pi} \frac{1}{r^{3}} \cdot \vec{L}^{2} \chi^{C}$ and satisfies $\left.F_{1}\right|_{\mathcal{S}_{00}}=0$. By Thms. 7.1.16 and 18 of [36] it follows that its Fourier transform $\hat{F}_{1}$ is homogeneous of degree 0 and restricts on $\mathbb{R}^{3} \backslash\{0\}$ to a smooth function, i.e., $\hat{F}_{1}(\vec{k})=\eta\left(\frac{\vec{k}}{|\vec{k}|}\right)$ for $\vec{k} \neq 0$ with some $\eta \in C^{\infty}\left(S^{2}\right)$. Moreover, since $\mathcal{S}_{00}$ is stable under the Fourier transform, one has $\left\langle Y_{00}, \eta\right\rangle=0$. Now consider the distribution $F_{2}:=-\Delta \Phi^{C}-F_{1}$. For $r \neq 0$, it is given by $F_{2}=\rho \cdot \chi^{C}+\left(\Phi-\frac{q_{\gamma}}{4 \pi r}\right) \frac{1}{r^{2}} \cdot \vec{L}^{2} \chi^{C}$ and thus has compact support. Hence its Fourier transform defines an analytic function $R(\vec{k})=\hat{F}_{2}(\vec{k})$ on $\mathbb{R}^{3}$. Thus both terms on the right-hand side of $-\widehat{\Delta \Phi^{C}}=\hat{F}_{1}+\hat{F}_{2}$ are smooth functions on $\mathbb{R}^{3} \backslash\{0\}$, which proves (for $\vec{k} \neq 0$ ) the identity $u^{C}(\vec{k})=-\widehat{\Delta \Phi^{C}}(\vec{k})=\eta(\vec{k} /|\vec{k}|)+R(\vec{k})$. To complete the proof, notice that $R(0)=\int d^{3} x\left(\rho \cdot \chi^{C}\right)(\vec{x})=0$, since $\chi^{C}$ was assumed to fulfil $\left\langle Y_{00}, \chi^{C}\right\rangle=0$.

Lemma 5.11 For any $f \in \mathcal{L}\left(\mathcal{C}^{\prime}\right)$, one has $l_{\gamma}(f)=-\lim _{n \rightarrow \infty} \operatorname{Im}\left\langle v_{n}^{C}, f\right\rangle$.

Proof: Any element $f \in \mathcal{L}\left(\mathcal{C}^{\prime}\right)$ has the form $f=\omega^{-\frac{1}{2}} \hat{h}+i \omega^{+\frac{1}{2}} \hat{g}$ with $h, g \in \mathcal{D}_{\mathbb{R}}\left(\mathbb{R}^{3} \backslash \bar{C}\right)$, whence $-\operatorname{Im}\left\langle v_{n}^{C}, f\right\rangle=\int_{|\vec{k}|>\epsilon_{n}} d^{3} k \omega^{-2} \overline{u^{C}(\vec{k})} \hat{h}(\vec{k})$. From Lemma 5.10 it follows in particular that $\widehat{\Phi^{C}}=\omega^{-2} u^{C}$ is locally integrable, so $-\operatorname{Im}\left\langle v_{n}^{C}, f\right\rangle$ converges for $n \rightarrow \infty$ to

$$
\int_{\mathbb{R}^{3}} d^{3} k \overline{\widehat{\Phi^{C}}(\vec{k})} \hat{h}(\vec{k})=\Phi^{C}(h)=\Phi(h) .
$$

Here we have viewed $\Phi^{C}$ and $\Phi$ as distributions and made use of the fact that they coincide on supph. The assertion now follows since $\Phi(h)=l_{\gamma}(f)$, as is easily seen.

With these preparations we obtain the following lemma which constitutes the main step in the proof of Prop. 5.9:

Lemma 5.12 Let $T$ be a KPR-like symplectic operator. Then:

i. The limit $v_{T}^{C}:=\lim _{n \rightarrow \infty} T v_{n}^{C}$ exists in $\mathcal{K}$.

ii. The unitary $W\left(v_{T}^{C}\right)$ satisfies

$$
\operatorname{Ad} W\left(v_{T}^{C}\right) \circ \pi_{T}=\pi_{T} \circ \gamma \quad \text { on } \quad \mathfrak{A}\left(\mathcal{C}^{\prime}\right) .
$$

Proof: i. Since $\Delta \Phi^{C}$ is real-valued, one has $\Gamma u^{C}=u^{C}$ and hence $\Gamma v_{n}^{C}=-v_{n}^{C}$. Therefore $T v_{n}^{C}=T_{1} v_{n}^{C}=i T_{1} \omega^{-\frac{3}{2}} P_{\epsilon_{n}} u^{C}$. Now $u^{C}$ can be written as a sum of vectors $u_{1}^{C}, u_{2}^{C} \in \mathcal{K}$ defined by $u_{1}^{C}:=\left(\mathbf{1}-P_{0}\right)(1 \cdot \eta)$ and $u_{2}^{C}:=u^{C}-u_{1}^{C}=P_{0}(1 \cdot \eta)+R$. Here $\eta$ and $R$ are as in Lemma 5.10. We show that the sequences $\left(T_{1} \omega^{-\frac{3}{2}} P_{\epsilon_{n}} u_{j}^{C}\right)_{n \in \mathbb{N}}(j=1,2)$ converge separately. For $j=1$, this is exactly the content of Lemma 5.7 (since $\left\langle Y_{00}, \eta\right\rangle=0$ ). For $j=2$, the behaviour of $R$ at the origin implies that $u_{2}^{C} \in D_{\omega^{-3 / 2}}$, whence it follows that $T_{1} \omega^{-\frac{3}{2}} P_{\epsilon_{n}} u_{2}^{C}=T_{1} P_{\epsilon_{n}} \omega^{-\frac{3}{2}} u_{2}^{C} \stackrel{n \rightarrow \infty}{\longrightarrow} T_{1} \omega^{-\frac{3}{2}} u_{2}^{C}$, since $T_{1}$ is bounded.

ii. Let $f \in \mathcal{L}\left(\mathcal{C}^{\prime}\right)$. Then $\operatorname{Im}\left\langle T v_{n}^{C}, T f\right\rangle=\operatorname{Im}\left\langle v_{n}^{C}, f\right\rangle$, which becomes in the limit $n \rightarrow \infty$ the equation $\operatorname{Im}\left\langle v_{T}^{C}, T f\right\rangle=-l_{\gamma}(f)$, due to Part i and to Lemma 5.11. This implies

$$
\begin{aligned}
\operatorname{Ad} W\left(v_{T}^{C}\right)\left(\pi_{T}(W(f))\right) & =W\left(v_{T}^{C}\right) W(T f) W\left(v_{T}^{C}\right)^{*}=e^{-i \operatorname{Im}\left\langle v_{T}^{C}, T f\right\rangle} W(T f) \\
& =e^{i l_{\gamma}(f)} W(T f)=\pi_{T} \circ \gamma(W(f)) .
\end{aligned}
$$


The stated equivalence is thus established on $\mathcal{W}\left(\mathcal{L}\left(\mathcal{C}^{\prime}\right)\right)$ and therefore (by the local normality of both $\pi_{T}$ and $\gamma$ ) also on $\mathfrak{A}\left(\mathcal{C}^{\prime}\right)$.

Proof of Prop. 5.9: Let $\gamma \in \mathcal{V}$ be arbitrary and let $\mathcal{C}$ be a spacelike cone with apex $x \in \mathbb{R}^{1+3}$. Then $\gamma \cong \tilde{\gamma}$ and $\mathcal{C}=x+\tilde{\mathcal{C}}$, where $\tilde{\gamma}$ and $\tilde{\mathcal{C}}$ have the special form considered above. Therefore one has $\operatorname{Ad} W\left(\tilde{v}_{T}^{C}\right) \circ \pi_{T}=\pi_{T} \circ \tilde{\gamma}$ on $\mathfrak{A}\left(\tilde{\mathcal{C}}^{\prime}\right)$ with some $\tilde{v}_{T}^{C} \in \mathcal{K}$. Now $\pi_{T}$ is translation covariant, so $\operatorname{Ad} U_{T}(x) \circ \pi_{T}=\pi_{T} \circ \alpha_{x}$ with a unitary $U_{T}(x)$, which implies $\operatorname{Ad}\left(U_{T}(x) W\left(\tilde{v}_{T}^{C}\right) U_{T}(x)^{*}\right) \circ \pi_{T}=\pi_{T} \circ \tilde{\gamma}_{x}$ on $\mathfrak{A}\left(\mathcal{C}^{\prime}\right)=\alpha_{x}\left(\mathfrak{A}\left(\tilde{\mathcal{C}}^{\prime}\right)\right)$. But since $\tilde{\gamma}_{x}=\alpha_{x} \circ \tilde{\gamma} \circ \alpha_{x}^{-1}$ has charge $q_{\gamma}$, it follows that $\left(\gamma-\tilde{\gamma}_{x}\right) \in D_{T}$, whence $\pi_{T} \circ \gamma=\operatorname{Ad} W\left(T\left(\gamma-\tilde{\gamma}_{x}\right)\right) \circ \pi_{T} \circ \tilde{\gamma}_{x}$ (on all of $\mathfrak{A}$ ). We thus have $\operatorname{Ad}\left(W\left(T\left(\gamma-\tilde{\gamma}_{x}\right)\right) U_{T}(x) W\left(\tilde{v}_{T}^{C}\right) U_{T}(x)^{*}\right) \circ \pi_{T}=\pi_{T} \circ \gamma$ on the algebra $\mathfrak{A}\left(\mathcal{C}^{\prime}\right)$, which was to be shown.

We have seen that choosing a background different from the vacuum can improve the localisability properties of the superselection sectors under consideration. For this to happen, the background must however be chosen carefully in order to match the sectors. Let us illustrate this by describing how a different choice of the symplectic operator $T$ would affect the result of Prop. 5.9:

- Assume that a $\left|Y_{00}\right\rangle\left\langle Y_{00}\right|$ term were present in each projection $\tilde{Q}_{i}$. Then the analogue of Lemma 5.7 would say that $\left(T_{1} \omega^{-\frac{3}{2}} P_{\epsilon_{n}} u\right)_{n \in \mathbb{N}}$ converges for arbitrary $\eta \in C^{\infty}\left(S^{2}\right)$. As a consequence, $\lim _{n} T P_{\epsilon_{n}} \gamma$ would exist for any $\gamma \in \mathcal{V}$, and one would immediately obtain $\pi_{T} \circ \gamma \cong \pi_{T}$ (on all of $\left.\mathfrak{A}\right)$ with the arguments of Lemma 5.12.

- Consider $\hat{T}=T_{2} \frac{1+\hat{\Gamma}}{2}+T_{1} \frac{1-\hat{\Gamma}}{2}$ instead of $T$, where $\hat{\Gamma}$ is complex conjugation in momentum space. Notice that $\hat{\Gamma}=\Gamma \circ(-1)^{\ell}$, where $(-1)^{\ell}$ is the parity operator (with eigenvalues \pm 1 on even/odd functions). If one tries to repeat the proof of Lemma 5.12 with $\hat{T}$, one sees from

$$
\hat{T} v_{n}^{C}=T_{2} \frac{1-(-1)^{\ell}}{2} v_{n}^{C}+T_{1} \frac{1+(-1)^{\ell}}{2} v_{n}^{C}
$$

that the odd part of $v_{n}^{C}$ is acted upon by the unbounded operator $T_{2}$. Thus $\hat{T} v_{n}^{C}$ can only converge if $v_{n}^{C}$ is even. This can be obtained by replacing $\chi^{C}$ with its even part $\chi^{ \pm C}$. As the latter satisfies $\left.\chi^{ \pm C}\right|_{S^{2} \backslash(C \cup-C)}=1$, it is clear from Lemmas 5.11 and 5.12 that the unitary $W\left(v_{\hat{T}}^{ \pm C}\right.$ ) (with $v_{\hat{T}}^{ \pm C}=\lim _{n} \hat{T} v_{n}^{ \pm C}$ defined in the obvious way) intertwines $\pi_{\hat{T}} \circ \gamma$ and $\pi_{\hat{T}}$ on $\mathfrak{A}\left(\mathcal{C}^{\prime} \cap-\mathcal{C}^{\prime}\right)$. This indicates that in front of the background $\pi_{\hat{T}}$ the sectors $[\gamma]$ can only be localised in (upright) opposite spacelike cones. A rigorous proof of this assertion can indeed be obtained along these lines.

For completeness, it has of course to be checked that the sectors are definitely not localisable in spacelike cones in front of $\pi_{\hat{T}}$. This is done with the standard method used in the proofs of Propositions 5.1 and 5.8: If $f=\omega^{-1 / 2} \hat{h} \in \mathcal{L}\left(\mathbb{R}^{3} \backslash C\right)$ has a rotation invariant even part, then $\left\|\hat{T} f_{\lambda}\right\| \leq\|f\|$ for all dilations $\lambda>0$, so the family $W\left(f_{\lambda}\right) \in \mathfrak{A}\left(\mathcal{C}^{\prime}\right)$ has a subsequence which converges weakly to different scalar multiples of 1 in the representations $\pi_{\hat{T}} \circ \gamma$ and $\pi_{\hat{T}}$.

- Localisation in upright opposite spacelike cones can also be obtained in front of $\pi_{T}$ (i.e., with the conjugation $\Gamma$ ): One has just to change the operators $T_{1}$ and $T_{2}$ by omitting in the projections $\tilde{Q}_{i}$ all summands $\left|Y_{\ell m}\right\rangle\left\langle Y_{\ell m}\right|$ with even angular momentum $\ell$. 
As a last remark we point out that the localisation property proved in Prop. 5.9 does not mean that $\pi_{T} \circ \gamma$ and $\pi_{T}$ are equivalent when restricted to causal complements of arbitrary spacelike cones. This is not surprising, since neither the KPR-like infravacuum representations $\pi_{T}$ nor the set $\mathcal{V}$ of automorphisms have a Lorentz symmetry, and it does in no way restrict the applicability of superselection theory, as we have seen in Chapter 3. It is quite likely, in fact, that $\pi_{T} \circ \gamma$ and $\pi_{T}$ are inequivalent (provided $q_{\gamma} \neq 0$ ) upon restriction to $\mathcal{C}^{\prime}$ if $\mathcal{C}$ is a spacelike cone which does not contain an upright one. It may be interesting to have an explicit proof of this along the standard arguments repeatedly used above. More speculatively, one might see from such a proof whether there exist modified KPRlike states in front of which the sectors $[\gamma]$ actually are localisable in arbitrary spacelike cones. 



\section{Chapter 6}

\section{Conclusions and Outlook}

In the present work it has been investigated which role infravacuum states may have for the description of quantum field theories with massless particles. Motivated from a qualitative understanding of QED, the basic hypothesis is that in these theories infravacua should replace the vacuum as a background in front of which charged states are being considered. In particular they ought to yield better localisability properties of the charges so as to render the DHR theory of superselection sectors applicable.

We have tested this hypothesis successfully in the example of the free massless scalar field. This model possesses a class of charged sectors which mimic the behaviour of electric charges. In particular they fulfil a sort of Gauss' law, which entails that they have very poor localisation properties in front of the vacuum. In contrast, they become localised in upright spacelike cones when seen in front of suitable background states of KPR type. The example shows clearly that the chosen background states must fit the sectors, otherwise either no improvement of the localisation is obtained, or (if the background is too strong) the charge becomes completely invisible. In special cases, pathologies such as localisation in opposite spacelike cones may arise as well.

The model of the free field is certainly very simple, and it would beyond any doubt be interesting to observe a similar localisation-improving mechanism in a theory whose kinematics is closer to that of QED. Possible candidates might be based on a model proposed by Herdegen [46].

In Chapter 2 we have analysed a possible definition of infravacuum states in arbitrary theories with massless particles. This definition expresses the idea that such states describe infrared clouds which may be approximated, up to arbitrarily small energy differences, by states belonging to the vacuum sector, i.e., by states with finitely many massless particles. As a consequence of this definition, one obtains a tight relation between the nets $\tilde{\mathcal{S}}_{\pi_{0}}(\cdot)$ and $\tilde{\mathcal{S}}_{\pi_{I}}(\cdot)$ of states with finite energy. It might be of technical interest to establish such a relation also in terms of exponential energy bounds (instead of the strict ones). This could lead to an alternative definition of infravacuum representations from which one can read off directly that it is fulfilled by the KPR representations. (We have seen in Chapter 5 that this is very likely to be the case, but a complete proof is still lacking.) Moreover one can ask whether such a definition can be refined so as to incorporate some quantitative notion measuring how "strong" a given infravacuum background is.

In Chapter 3 we have reviewed the DHR theory of superselection sectors for charges with general localisation properties. We have paid particular attention to the topological properties of the system of localisation regions, and chosen a consequently functorial for- 
mulation. Charges covariant under some spacetime symmetry group are best described by charge transporting cocycles, the algebraic properties of which we have collected. The classification of these cocycles seems to lead to interesting questions in nonabelian group cohomology.

Finally, we have investigated whether the energy-momentum properties of superselection charges in front of an infravacuum background are the ones known from the vacuum case. Here a fundamental distinction has to be made between compactly localised charges and charges localised in spacelike cones, say. For the former, the superselection structure is independent of the chosen background. This concerns not only the algebraic structure (thus there are no infravacuum backgrounds in front of which some charges are invisible), but more importantly also the energy-momentum spectra (under mild assumptions on the infravacuum). For instance, the masses of charge-carrying particles do not depend on the background.

In the case of non-compactly localised charges, the situation is radically different: there is no a priori way to even compare the charges appearing in front of different backgrounds. As a consequence the very question whether the mass of a given charge-carrying particle or the interaction between two charges depends on the background does not make sense. A question which is meaningful, on the contrary, is whether (like in front of the vacuum) the existence of a conjugate implies for a sector to have positive energy. This is to be expected as a consequence of the infravacuum background containing a finite amount of energy. A proof of this however requires a better control of the spacelike asymptotic properties of infravacuum states than what is known at present.

Another interesting question is if there is some PCT symmetry in front of infravacuum backgrounds in the sense that the energy-momentum spectra associated to a sector and to its conjugate coincide. This cannot yet be answered in an affirmative way, but what could be established is that the minimal energy-momentum operators associated to pairs of conjugate sectors are transformed into each other under charge conjugation. (This is a nontrivial issue since the charge conjugation is defined algebraically, whereas the energymomentum operator is characterised by analytic properties.) To obtain this result, we have generalised in Appendix D the Jost-Lehmann-Dyson method from [8]. It seems likely that related methods of analytic continuation can actually be used to give a positive answer to the above question.

Summarising, we have seen that infravacua have important properties which make them well-suited for the description of quantum field theories with long-range forces. Their precise mathematical properties, in particular the ones regarding the sense in which they are "energetically close" to the vacuum nevertheless still need further investigation. 


\section{Appendix A}

\section{Some Category Theory}

We want to collect in this appendix the definitions and basic properties of certain monoidal $\mathrm{C}^{*}$-categories and introduce the notions of conjugates, symmetry and left inverses. Most of these topics are covered by [2.9, 37, 38]. For elementary general notions such as, e.g., that of categories, functors and natural transformations, we refer to the standard monograph [39].

\section{A.1 $\mathrm{C}^{*}-, \mathrm{W}^{*}$ - and Monoidal Categories}

Let $\mathcal{C}$ be a (small) category. We will write $\rho \in \mathcal{C}$ if $\rho$ is an object of $\mathcal{C}$ and denote (for $\sigma, \tau \in \mathcal{C}$ ) with $I(\sigma, \tau)$ the set of morphisms (or arrows) from $\sigma$ to $\tau$. The notation $t: \sigma \rightarrow \tau$ will be used synonymously to $t \in I(\sigma, \tau)$. The identity morphisms are denoted with $\mathbf{1}_{\rho}$, $\rho \in \mathcal{C}$, and the composition of morphisms with $\circ$ or, occasionally, with no symbol at all. Invertible morphisms are called isomorphisms, and two objects $\sigma$ and $\tau$ are said to be isomorphic (written $\sigma \cong \tau$ ) if there exists an isomorphism between them.

If $\mathcal{C}$ is a category and $\mathcal{C}_{0}$ is a collection of objects and morphisms of $\mathcal{C}$ closed under o and containing all $\mathbf{1}_{\rho}, \rho \in \mathcal{C}_{0}$, then $\mathcal{C}_{0}$ is called a subcategory of $\mathcal{C}$. It is called a full subcategory if it contains all morphisms $t \in I(\sigma, \tau), \quad \sigma, \tau \in \mathcal{C}_{0}$. Thus a full subcategory of $\mathcal{C}$ is specified by a subset of the objects only, and we therefore write $\mathcal{C}_{0} \subset \mathcal{C}$ in this case.

Definition: A category $\mathcal{C}$ is a $C^{*}$-category if all sets $I(\sigma, \tau), \sigma, \tau \in \mathcal{C}$ are Banach spaces over $\mathbb{C}$ (with addition + and norm $\|\cdot\|$ ) and if there is an antilinear involutive *-operation $*: I(\sigma, \tau) \longrightarrow I(\tau, \sigma): t \longmapsto t^{*}$ satisfying $(s \circ t)^{*}=t^{*} \circ s^{*}$ in such a way that

- the composition $\circ$ is bilinear,

- $\|s \circ t\| \leq\|s\|\|t\|$,

- $\left\|t^{*} \circ t\right\|=\|t\|^{2}$.

(Taken together, $(+,\|\cdot\|, *)$ is sometimes called the $C^{*}$-structure of $\mathcal{C}$.)

The notions of self-adjoint morphisms and (orthogonal) projections, partial isometries, isometries and unitaries are defined in an obvious way. For any $\rho \in \mathcal{C}, I(\rho, \rho)$ is a $\mathrm{C}^{*}$ algebra, and $\rho$ is said to be irreducible if $I(\rho, \rho)=\mathbb{C} \mathbf{1}_{\rho}$. Two objects $\rho_{1}, \rho_{2} \in \mathcal{C}$ are called equivalent if $I\left(\rho_{1}, \rho_{2}\right)$ contains a unitary $u: \rho_{1} \rightarrow \rho_{2}$, and $\sigma$ is said to be a subobject of $\rho$ if $I(\sigma, \rho)$ contains an isometry $w: \sigma \hookrightarrow \rho$. (By polar decomposition, it is easy to see that 
Appendix A. Some Category Theory

equivalence of two objects it tantamount to isomorphism and that the above definition of subobjects reproduces the more general one in terms of a certain universal property.)

An object $\rho$ is called a direct sum of $\rho_{1}, \ldots, \rho_{N}$ if there exist isometries $w_{j}: \rho_{j} \hookrightarrow \rho$ $(j=1, \ldots, N)$ whose final projections sum up to the identity, i.e., $\sum_{j} w_{j} w_{j}^{*}=\mathbf{1}_{\rho}$. The $\mathrm{C}^{*}$-category $\mathcal{C}$ is said to be closed under subobjects if any projection is the final projection of some isometry and closed under finite direct sums if, given $\rho_{1}, \ldots, \rho_{N} \in \mathcal{C}$, there is some $\rho \in \mathcal{C}$ which is their direct sum. We will write $\bigoplus_{j} \rho_{j}$ for such an object, bearing in mind that it is unique only up to equivalence.

Definition: A $\mathrm{C}^{*}$-category $\mathcal{C}$ is called a $W^{*}$-category if each Banach space $I(\sigma, \tau)$ has a predual, i.e., if it is the dual of some Banach space $I(\sigma, \tau)_{*}$.

It can be shown that these preduals $I(\sigma, \tau)_{*}$ are unique (as subspaces of the dual $I(\sigma, \tau)^{*}$ of $\left.I(\sigma, \tau)\right)$. As a consequence, the spaces $I(\sigma, \tau)$ possess a natural topology, usually denoted with $\sigma\left(I(\sigma, \tau), I(\sigma, \tau)_{*}\right)$, induced from this predual, which we will refer to in the sequel as the $\mathrm{w}^{*}$-topology. (Also, it is sometimes useful to subsume under the name $\mathrm{W}^{*}$-structure the $\mathrm{C}^{*}$-structure and the $\mathrm{w}^{*}$-topologies on all spaces $I(\sigma, \tau)$.) It should be noted that the maps $t \longmapsto t^{*}, t \longmapsto t \circ y$ and $t \longmapsto y^{\prime} \circ t$ (for fixed morphisms $y$ and $y^{\prime}$ ) are $\mathrm{w}^{*}$-continuous, which is analogous to the situation in $\mathrm{W}^{*}$-algebras.

Example: The basic example of $\mathrm{C}^{*}$ - and $\mathrm{W}^{*}$-categories relevant to the main text is of the following type. If $\mathcal{A}$ and $\mathcal{B}$ are unital $\mathrm{C}^{*}$-algebras, let $\operatorname{Hom}(\mathcal{A}, \mathcal{B})$ be the category whose objects are the unital ${ }^{*}$-homomorphisms $\rho: \mathcal{A} \longrightarrow \mathcal{B}$ and whose sets of morphisms are given by

$$
I(\sigma, \tau):=\{t=(\tau, t, \sigma) \mid t \in \mathcal{B}, \tau(a) t=t \sigma(a) \quad \text { for all } a \in \mathcal{A}\} .
$$

Morphisms are composed according to $(\tau, t, \sigma) \circ(\sigma, s, \rho)=(\tau, \underline{t}, \rho, \rho)$, and the identity morphisms are $\mathbf{1}_{\rho}=(\rho, \mathbf{1}, \rho)$. For each pair $\sigma, \tau$, the image $I(\sigma, \tau)$ of the canonical map $I(\sigma, \tau) \longrightarrow \mathcal{B}: t \longmapsto t$ is a Banach subspace of $\mathcal{B}$, and $I(\sigma, \tau)$ can thus be made into a Banach space in the obvious way. With $(\tau, t, \sigma)^{*}=\left(\sigma, t^{*}, \tau\right)$ as the ${ }^{*}$-operation, $\operatorname{Hom}(\mathcal{A}, \mathcal{B})$ is readily seen to be a $\mathrm{C}^{*}$-category. In particular, note that $I(\rho, \rho)=\mathcal{B} \cap \rho(\mathcal{A})^{\prime}$.

Now if $\mathcal{B}$ is even a $\mathrm{W}^{*}$-algebra, i.e., the dual of a Banach space $\mathcal{B}_{*}$, then each $I(\sigma, \tau)$ is the dual of the Banach space $I(\sigma, \tau)_{*}:=\mathcal{B}_{*} / I^{\circ}$, where $I^{\circ}:=\left\{l \in \mathcal{B}_{*}|l|_{I(\sigma, \tau)}=0\right\}$. Therefore each $I(\sigma, \tau)$, too, has a predual, which means that $\operatorname{Hom}(\mathcal{A}, \mathcal{B})$ is a $\mathrm{W}^{*}$-category. The $\mathrm{w}^{*}$-topology on $I(\sigma, \tau)$ can be characterised directly by saying that a net $\left(t_{\alpha}\right)$ in $I(\sigma, \tau)$ converges to some $t \in I(\sigma, \tau)$ iff $l\left(t_{\alpha}\right) \stackrel{\alpha}{\longrightarrow} l(t)$ for every $l \in \mathcal{B}_{*}$. It is thus the pullback of the $\mathrm{w}^{*}$-topology of $\mathcal{B}$ via the map $I(\sigma, \tau) \longrightarrow \mathcal{B}: t \longmapsto t$. Notice that we have a slightly more powerful structure here not covered by the definition of $\mathrm{W}^{*}$-categories: if we denote with MorC $=\bigcup_{\sigma, \tau} I(\sigma, \tau)$ the (disjoint) union of all sets of morphisms, then the pullback of the $\mathrm{w}^{*}$-topology of $\mathcal{B}$ via the map MorC $\longrightarrow \mathcal{B}: t \longmapsto t$ defines a topology on MorC (we might call it the overall $\mathrm{w}^{*}$-topology) which generates the $\mathrm{w}^{*}$-topology of each "fibre" $I(\sigma, \tau)$ as a subspace topology, but which is smaller than the "fibrewise discrete" topology. In particular there exist $\mathrm{w}^{*}$-continuous morphism-valued functions $\xi \longmapsto t_{\xi}=\left(\tau_{\xi}, t_{\xi}, \sigma_{\xi}\right)$, $\xi \in \mathbb{R}$ with non-constant functions $\xi \longmapsto \sigma_{\xi}, \tau_{\xi}$. (This plays a certain role in Section 3.7). It should be remarked that the overall $\mathrm{w}^{*}$-topology is non-Hausdorff (not even $\mathrm{T}_{0}$ ); in restriction to the set $\left\{\mathbf{1}_{\rho} \mid \rho \in \mathcal{C}\right\}$, it merely generates the chaotic topology.

Definition: A category $\mathcal{C}$ equipped with an associative binary operation $\cdot$ on the set of objects, $\left(\rho_{1}, \rho_{2}\right) \longmapsto \rho_{1} \cdot \rho_{2}$, and an associative binary operation $\otimes$ on the set of 
morphisms, $\left(t_{1}, t_{2}\right) \longmapsto t_{1} \otimes t_{2}$, is called a monoidal category if the following conditions are fulfilled:

- for $t_{1}: \sigma_{1} \rightarrow \tau_{1}$ and $t_{2}: \sigma_{2} \rightarrow \tau_{2}$, one has $t_{1} \otimes t_{2}: \sigma_{1} \cdot \sigma_{2} \rightarrow \tau_{1} \cdot \tau_{2}$

- $\mathbf{1}_{\rho_{1} \otimes} \mathbf{1}_{\rho_{2}}=\mathbf{1}_{\rho_{1} \cdot \rho_{2}}$;

- $\left(s_{1} \circ t_{1}\right) \otimes\left(s_{2} \circ t_{2}\right)=\left(s_{1} \otimes s_{2}\right) \circ\left(t_{1} \otimes t_{2}\right)$ whenever the left-hand side is defined;

- there exists some $\iota \in \mathcal{C}$ which is the neutral element of $\cdot$ and such that $\mathbf{1}_{\iota}$ is the neutral element of $\otimes$.

The operations $\cdot$ and $\otimes$ are called the monoidal products since they make the sets of objects (resp. of morphisms) into monoids (i.e., semigroups with unit); the object $\iota$ is called the monoidal unit. The triple $(\cdot, \otimes, \iota)$ is referred to as the monoidal structure of $\mathcal{C}$. It is easily seen that both $\otimes$ and $\circ$ coincide and are abelian on $I(\iota, \iota)$.

To economize on brackets, it is customary to evaluate $\otimes$ before $\circ$. Moreover, we adopt the additional convention that an omitted composition sign is to be evaluated before $\otimes$. Thus, $r \otimes s t \circ u=(r \otimes(s \circ t)) \circ u$, and $\left(s_{1} \circ t_{1}\right) \otimes\left(s_{2} \circ t_{2}\right)=\left(s_{1} \otimes s_{2}\right) \circ\left(t_{1} \otimes t_{2}\right)$ becomes $s_{1} t_{1} \otimes s_{2} t_{2}=$ $s_{1} \otimes s_{2} \circ t_{1} \otimes t_{2}$. Also, we will frequently omit the symbol $\cdot$, writing $\rho_{1} \rho_{2}$ for $\rho_{1} \cdot \rho_{2}$.

If a category $\mathcal{C}$ has a $\mathrm{C}^{*}$-structure as well as a monoidal structure, the following compatibility conditions are natural:

Definition: A category $\mathcal{C}$ which is both a $\mathrm{C}^{*}$-category and a monoidal category is called a monoidal $C^{*}$-category if the monoidal product $\otimes$ is bilinear and fulfils

$$
\left(t_{1} \otimes t_{2}\right)^{*}=t_{1}^{*} \otimes t_{2}^{*}
$$

Note that the monoidal product is automatically $\|\cdot\|$-continuous since $\left\|t_{1} \otimes t_{2}\right\| \leq$ $\left\|t_{1}\right\|\left\|t_{2}\right\|$. (This inequality follows from the $\mathrm{C}^{*}$-property of the norm and from the fact that endomorphisms of $\mathrm{C}^{*}$-algebras are contracting.) In contrast to this (and in the more special case that the $\mathrm{C}^{*}$-category is even a $\mathrm{W}^{*}$-category), $\mathrm{w}^{*}$-continuity of the monoidal product is not automatically fulfilled. We therefore introduce the following notion:

Definition: A category $\mathcal{C}$ which is both a $\mathrm{W}^{*}$-category and a monoidal $\mathrm{C}^{*}$-category is called a monoidal $W^{*}$-category if for any $\rho \in \mathcal{C}$, the maps

$$
t \longmapsto t \otimes \mathbf{1}_{\rho} \quad \text { and } \quad t \longmapsto \mathbf{1}_{\rho} \otimes t
$$

are $\mathrm{w}^{*}$-continuous on each set $I(\sigma, \tau)$ of morphisms.

As a consequence, in a monoidal $\mathrm{W}^{*}$-category the product

$$
\left(t_{1}, t_{2}\right) \longmapsto t_{1} \otimes t_{2}=t_{1} \otimes \mathbf{1}_{\tau_{2}} \circ \mathbf{1}_{\sigma_{1}} \otimes t_{2}
$$

(where $\sigma_{1}$ is the source of $t_{1}$ and $\tau_{2}$ the target of $t_{2}$ ) is $\mathrm{w}^{*}$-continuous in each entry. Notice that the two continuity properties demanded in the definition are unrelated in general; they are equivalent, however, if $\mathcal{C}$ admits a symmetry (cf. below, or more generally a braiding).

Example: Let us illustrate these notions in the setting of the above example. If $\mathcal{A}$ is a unital $\mathrm{C}^{*}$-algebra, then it can be verified in a straightforward manner that the

\footnotetext{
${ }^{1}$ Since relaxed monoidal categories will not be considered, the term "monoidal" will be taken as synonymous to "strict monoidal".
} 
$\mathrm{C}^{*}$-category End $\mathcal{A}:=\operatorname{Hom}(\mathcal{A}, \mathcal{A})$ becomes a monoidal $\mathrm{C}^{*}$-category when equipped with the composition of endomorphisms of $\mathcal{A}$ as the monoidal product of objects and with the operation

$$
\left(\tau_{1}, t_{1}, \sigma_{1}\right) \otimes\left(\tau_{2}, t_{2}, \sigma_{2}\right):=\left(\tau_{1} \tau_{2}, t_{1} \sigma_{1}\left(t_{2}\right), \sigma_{1} \sigma_{2}\right)
$$

as that of morphisms. If $\mathcal{A}$ is even a $\mathrm{W}^{*}$-algebra, then the $\mathrm{w}^{*}$-continuity of the map $t=(\tau, t, \sigma) \longmapsto t \otimes \mathbf{1}_{\rho}=(\tau \rho, t, \sigma \rho)$ is trivial while that of $t \longmapsto \mathbf{1}_{\rho} \otimes t=(\rho \tau, \rho(t), \rho \sigma)$ may fail unless $\rho$ is normal. So in general only the full subcategory of normal endomorphisms of $\mathcal{A}$ is a monoidal $\mathrm{W}^{*}$-category.

Another example almost of the same spirit (and of some peripheral importance at the very end of Section 3.7) is that of endofunctors of a given category $\mathcal{C}$. An endofunctor of $\mathcal{C}$ is nothing but a functor $\beta: \mathcal{C} \longrightarrow \mathcal{C}$, and a natural transformation $\mu: \beta \Rightarrow \beta^{\prime}$ between two such functors is a family $\left(\mu_{\rho}\right)_{\rho \in \mathcal{C}}$ of morphisms $\mu_{\rho}: \beta(\rho) \rightarrow \beta^{\prime}(\rho)$ which intertwines these functors in the sense that $\mu_{\tau} \circ \beta(t)=\beta^{\prime}(t) \circ \mu_{\sigma}$ for all $t: \sigma \rightarrow \tau$. The endofunctors of $\mathcal{C}$ as objects together with the natural transformations as morphisms form the category EndC . The latter can be equipped with the monoidal structure $\left(\cdot, \otimes, \operatorname{Id}_{\mathcal{C}}\right)$ : $\operatorname{Id}_{\mathcal{C}}$ is the identity functor, · the composition of functors, and the "horizontal product" $\mu \otimes \nu: \beta \gamma \Rightarrow \beta^{\prime} \gamma^{\prime}$ of two natural transformation $\mu: \beta \Rightarrow \beta^{\prime}$ and $\nu: \gamma \Rightarrow \gamma^{\prime}$ is given by the formula $(\mu \otimes \nu)_{\rho}=\mu_{\gamma^{\prime}(\rho)} \circ \beta\left(\nu_{\rho}\right)=\beta^{\prime}\left(\nu_{\rho}\right) \circ \mu_{\gamma(\rho)}$.

To all of the above kinds of categories, there is a corresponding notion of subcategory: A subcategory $\mathcal{C}_{0}$ of a $\mathrm{C}^{*}-/ \mathrm{W}^{*_{-}} /$monoidal category $\mathcal{C}$ is a $\mathrm{C}^{*_{-}} / \mathrm{W}^{*_{-}} /$monoidal subcategory of $\mathcal{C}$ if it is a $\mathrm{C}^{*_{-}} / \mathrm{W}^{*_{-}} /$monoidal category with the same $\mathrm{C}^{*_{-}} / \mathrm{W}^{*_{-}} /$monoidal structure as $\mathcal{C}$. (As a consequence, a $\mathrm{C}^{*}-\mathrm{W}^{*}$-subcategory which is also a monoidal subcategory is a monoidal $\mathrm{C}^{*}-/ \mathrm{W}^{*}$-subcategory.) These conditions involve objects and morphisms, but they become particularly simple in the case of full subcategories: If $\mathcal{C}_{0} \subset \mathcal{C}$ is a full subcategory of a $\mathrm{C}^{*}-/ \mathrm{W}^{*}$-category $\mathcal{C}$, then it is automatically a $\mathrm{C}^{*}$ - $/ \mathrm{W}^{*}$-subcategory; if $\mathcal{C}_{0} \subset \mathcal{C}$ is a full subcategory of a monoidal category $\mathcal{C}$, then it is a monoidal subcategory of $\mathcal{C}$ if $\iota \in \mathcal{C}_{0}$ and if $\rho_{1} \cdot \rho_{2} \in \mathcal{C}_{0}$ for any two objects $\rho_{1}, \rho_{2} \in \mathcal{C}_{0}$.

For all of the above kinds of categories, there is also an appropriate kind of functor: While a general functor $F: \mathcal{C}_{1} \longrightarrow \mathcal{C}_{2}$ is only required to map objects (resp. morphisms) of $\mathcal{C}_{1}$ to objects (resp. morphisms) of $\mathcal{C}_{2}$ in a way which preserves the unit morphisms and the composition, a $\mathrm{C}^{*_{-}} / \mathrm{W}^{*_{-}} /$monoidal functor between two $\mathrm{C}_{-}^{*_{-}} / \mathrm{W}^{*_{-}} /$monoidal categories must in addition preserve the $\mathrm{C}^{*}-/ \mathrm{W}^{*}-/$ monoidal structure too. (In the $\mathrm{C}^{*}$ case, this means that $F$ must be linear and fulfil $F\left(t^{*}\right)=F(t)^{*}$; it is then automatically bounded: $\|F(t)\| \leq\|t\|$.) The definition of monoidal $\mathrm{C}^{*}-/ \mathrm{W}^{*}$-functors involves no additional conditions between the two structures. The simplest examples for these sorts of functors are given by the inclusion functors of a subcategory $\mathcal{C}_{0}$ into the ambient one $\mathcal{C}$ discussed in the previous paragraph. Another example (of some relevance to Chapter 3) are $\mathrm{C}^{*}$-functors between $\mathrm{C}^{*}$-categories of the type $\operatorname{Hom}(\mathcal{A}, \mathcal{B})$ : in a natural way, two unital $\mathrm{C}^{*}$-algebra homomorphisms $\alpha: \mathcal{A}_{0} \longrightarrow \mathcal{A}_{1}$ and $\beta: \mathcal{B}_{1} \longrightarrow \mathcal{B}_{2}$ yield a $\mathrm{C}^{*}$-functor $\operatorname{Hom}\left(\mathcal{A}_{1}, \mathcal{B}_{1}\right) \longrightarrow \operatorname{Hom}\left(\mathcal{A}_{0}, \mathcal{B}_{2}\right)$. We leave the details to the reader. For completeness, let us mention that the above functors acquire the prefix "iso-" if they have inverses of the same kind and "auto-" if in addition their range coincides with their domain.

For the sake of brevity, it seems advantageous to adopt the following specific (nonstandard) denomination for the sort of categories which will be dealt with very often in the sequel and in the main text. 
Definition: A monoidal $\mathrm{C}^{*}\left(\right.$ resp. $\left.\mathrm{W}^{*}\right)$-category with irreducible monoidal unit and closed under subobjects and finite direct sums will be called an $\mathrm{rC}^{*}\left(\right.$ resp. $\left.\mathrm{rW}^{*}\right)$-category.

(The letter " $\mathrm{r}$ " is intended to be a mnemonic inspired by the words "ring" and "reduction", the former recalling that the set of objects in such a category is indeed a (semi)ring, the latter standing for the fact that any object can be decomposed into irreducibles.)

\section{A.2 Conjugates, Left Inverses and Symmetry}

We will now recall the notions of conjugates, left inverses and of symmetry in such categories. All three items are related to the monoidal product, but none of them need exist in an arbitrary monoidal category. Nevertheless, they are not independent of each other; in particular, the existence of conjugates implies that of left inverses. In the application to superselection theory the crucial point is that the presence of a symmetry induces a certain converse of this statement: the existence of left inverses allows one to single out certain objects with "finite statistics" and, among them, a special class of "simple" objects and to show that the former have conjugates if only the latter do. (This will be reviewed in Section A.3.)

Let us begin with the notion of conjugates which is an important concept in the theory of monoidal categories. In the particular case of monoidal $\mathrm{C}^{*}$-categories, it is best introduced as follows:

Definition: Let $\mathcal{C}$ be a monoidal $\mathrm{C}^{*}$-category. Then two objects $(\rho, \bar{\rho})$ of $\mathcal{C}$ are said to be conjugate to each other if there exist morphisms $r: \iota \rightarrow \bar{\rho} \rho$ and $\bar{r}: \iota \rightarrow \rho \bar{\rho}$ such that

$$
\bar{r}^{*} \otimes \mathbf{1}_{\rho} \circ \mathbf{1}_{\rho} \otimes r=\mathbf{1}_{\rho} \quad \text { and } \quad r^{*} \otimes \mathbf{1}_{\bar{\rho}} \circ \mathbf{1}_{\bar{\rho} \otimes \bar{r}}=\mathbf{1}_{\bar{\rho}} .
$$

The pair $(r, \bar{r})$ is called a solution of the conjugate equations for $(\rho, \bar{\rho})$, and we will write $\mathcal{R}(\rho, \bar{\rho})$ for the set of all these solutions. The number $d(\rho):=\inf \{\|r\|\|\bar{r}\| \mid(r, \bar{r}) \in \mathcal{R}(\rho, \bar{\rho})\}$ is called the dimension of $\rho$. Objects which possess a conjugate are called finite dimensional, and the full subcategory of all finite dimensional objects will be denoted with $\mathcal{C}_{\text {f.d. }}$.

If it exists, such a conjugate $\bar{\rho}$ of a given $\rho$ is unique up to unitary equivalence; for a given pair $(\rho, \bar{\rho})$ of conjugate objects, the above morphisms $r$ and $\bar{r}$ determine each other uniquely. Obviously, $d(\rho)=d(\bar{\rho})$, and equivalent objects have the same dimension.

In the special context of $\mathrm{rC}^{*}$-categories, $\mathcal{C}_{\text {f.d. }}$ has been studied extensively in [37]. It was shown, in particular, that $\mathcal{C}_{\text {f.d. }}$ is an $\mathrm{rC}^{*}$-subcategory and that an object $\rho \in \mathcal{C}$ is finite dimensional iff it is a finite direct sum of irreducibles from $\mathcal{C}_{\text {f.d. }}$. Moreover, it was shown that the dimension $d$ is additive and multiplicative and that it takes its values in (a certain subset of) the interval $[1, \infty[$.

We will need some more specific notions (such as that of standard solutions and a certain conjugation on objects and morphisms) in the main text. In order to avoid too long a digression at this point, we postpone the summary of these properties to Section A.4.

For the purpose of identifying the finite dimensional objects from within a given $\mathrm{rC}^{*}$ category $\mathcal{C}$, the somewhat weaker notion of left inverses turns out to be extremely useful. This concept goes back to [7], but we will adopt here the more modern formulation of [37].

Definition: Let $\mathcal{C}$ be a monoidal $\mathrm{C}^{*}$-category and $\rho$ an object of $\mathcal{C}$. Then a family $\varphi=\left(\varphi_{\sigma, \tau}\right)_{\sigma, \tau \in \mathcal{C}}$ of bounded linear maps $\varphi_{\sigma, \tau}: I(\rho \sigma, \rho \tau) \longrightarrow I(\sigma, \tau)$ is called a left inverse of $\rho$ if the following conditions are fulfilled: 
1. $\varphi_{\sigma^{\prime}, \tau^{\prime}}\left(\mathbf{1}_{\rho} \otimes t \circ y \circ \mathbf{1}_{\rho^{\otimes}} s^{*}\right)=t \circ \varphi_{\sigma, \tau}(y) \circ s^{*}$ for all $t \in I\left(\tau, \tau^{\prime}\right), s \in I\left(\sigma, \sigma^{\prime}\right), y \in I(\rho \sigma, \rho \tau)$;

2. $\varphi_{\sigma \pi, \tau \pi}\left(y \otimes \mathbf{1}_{\pi}\right)=\varphi_{\sigma, \tau}(y) \otimes \mathbf{1}_{\pi} \quad$ for all $y \in I(\rho \sigma, \rho \tau)$;

3. $\varphi_{\iota, \iota}\left(\mathbf{1}_{\rho}\right)=\mathbf{1}_{\iota}$;

4. every map $\varphi_{\sigma, \sigma}, \sigma \in \mathcal{C}$ is positive.

The set of all left inverses of $\rho$ will be denoted with $\operatorname{LI}(\rho)$.

The existence of left inverses is necessary for the existence of a conjugate. More explicitly, if $(r, \bar{r}) \in \mathcal{R}(\rho, \bar{\rho})$, then every positive element $m \in I(\bar{\rho}, \bar{\rho})$ gives rise to a left inverse $\varphi \in \operatorname{LI}(\rho)$ by the formula $\varphi_{\sigma, \tau}(t)=\frac{1}{N} r^{*}{ }_{\otimes} \mathbf{1}_{\tau} \circ m \otimes t \circ r \otimes \mathbf{1}_{\sigma}$, where $N>0$ is the obvious normalisation constant. (Moreover, every element of $\operatorname{LI}(\rho)$ arises in this way, whence one can establish a bijection between $\operatorname{LI}(\rho)$ and the set of properly normalised positive elements of $I(\bar{\rho}, \bar{\rho})$.)

If the monoidal $\mathrm{C}^{*}$-category $\mathcal{C}$ is a $\mathrm{W}^{*}$-category, then the sets $\operatorname{LI}(\rho), \rho \in \mathcal{C}$ can be equipped with the pointwise $\mathrm{w}^{*}$-topology induced from each set $I(\sigma, \tau)$ of morphisms. Explicitly, a (generalised) sequence $\varphi^{(\alpha)} \in \operatorname{LI}(\rho)$ is defined to converge to some $\varphi \in \operatorname{LI}(\rho)$ iff

$$
\mathrm{w}^{*}-\lim _{\alpha} \varphi_{\sigma, \tau}^{(\alpha)}(t)=\varphi_{\sigma, \tau}(t) \quad \text { for all } t \in I(\rho \sigma, \rho \tau) \text { and all } \sigma, \tau \in \mathcal{C} .
$$

In the special case when the monoidal product is $\mathrm{w}^{*}$-continuous so as to comply with the definition of a monoidal $\mathrm{W}^{*}$-category, this topology has the following property important for the present purposes:

Lemma A.1 If $\mathcal{C}$ is a monoidal $W^{*}$-category and $\rho \in \mathcal{C}$, then $\operatorname{LI}(\rho)$ is compact in the pointwise $w^{*}$-topology.

Proof: $\operatorname{LI}(\rho)$ is, in a natural way, a subspace of $B_{1}:=\prod_{\sigma, \tau} B(\sigma, \tau)_{1}$, where $B(\sigma, \tau)_{1}$ denotes the unit ball in the set $B(\sigma, \tau)$ of all bounded linear maps from $I(\rho \sigma, \rho \tau)$ to $I(\sigma, \tau)$. Now if $\mathcal{C}$ is a $\mathrm{W}^{*}$-category, the unit ball of each $I(\sigma, \tau)$ is $\mathrm{w}^{*}$-compact, therefore it follows by the arguments of [3] that each $B(\sigma, \tau)_{1}$ is compact in the pointwise $\mathrm{w}^{*}$ topology, so by Tychonoff's theorem, $B_{1}$ is compact, too. Since the pointwise w*-topology is Hausdorff, the assertion follows if $\operatorname{LI}(\rho)$ is seen to be a closed subset. Thus, let $\varphi^{(\alpha)} \in$ $\operatorname{LI}(\rho)$ be a (generalised) sequence which converges to some $\varphi \in B_{1}$. Then it can be shown that $\varphi$ fulfils the four defining properties of left inverses: 1 and 2 follow from the corresponding properties of the $\varphi^{(\alpha)}$ because the composition $\circ$ and the map $y \longmapsto y \otimes \mathbf{1}_{\pi}$ are $\mathrm{w}^{*}$-continuous (in each entry). $\varphi_{\iota, \iota}\left(\mathbf{1}_{\rho}\right)=\mathbf{1}_{\iota}$ is trivial, and the positivity of each $\varphi_{\sigma, \sigma}$ follows from that of the maps $\varphi_{\sigma, \sigma}^{(\alpha)}$ and from the fact that the set of positive elements in the $\mathrm{W}^{*}$-algebra $I(\sigma, \sigma)$ is $\mathrm{w}^{*}$-closed.

The last notion to be introduced is that of a symmetry.

Definition: A symmetry in a monoidal $\mathrm{C}^{*}$-category $\mathcal{C}$ is a family $\epsilon=(\epsilon(\sigma, \tau))_{\sigma, \tau \in \mathcal{C}}$ of unitaries $\epsilon(\sigma, \tau): \sigma \tau \rightarrow \tau \sigma$ which satisfy

$$
\begin{gathered}
\epsilon(\sigma, \tau)^{*}=\epsilon(\tau, \sigma), \\
\epsilon(\iota, \rho)=\epsilon(\rho, \iota)=\mathbf{1}_{\rho}, \\
\epsilon(\sigma \rho, \tau)=\epsilon(\sigma, \tau) \otimes \mathbf{1}_{\rho} \circ \mathbf{1}_{\sigma} \otimes \epsilon(\rho, \tau)
\end{gathered}
$$


and, for any $t_{j}: \sigma_{j} \rightarrow \tau_{j}(j=1,2)$,

$$
\epsilon\left(\tau_{1}, \tau_{2}\right) \circ t_{1} \otimes t_{2}=t_{2} \otimes t_{1} \circ \epsilon\left(\sigma_{1}, \sigma_{2}\right) .
$$

A symmetry $\epsilon$ gives rise to some more structure. Namely, to any fixed $\rho \in \mathcal{C}$, there is associated in a canonical way a sequence $\epsilon_{\rho}^{(n)}: \mathbb{C P}_{n} \longrightarrow I\left(\rho^{n}, \rho^{n}\right), n \in \mathbb{N}$ of (unital $\mathrm{C}^{*}$-algebra) homomorphisms, $\mathbb{C P}_{n}$ denoting the group algebra of the permutation group $\mathbb{P}_{n}$, in such a way that ${ }^{2}$

$$
\epsilon_{\rho}^{(n)}(p) \otimes \epsilon_{\rho}^{(m)}(q)=\epsilon_{\rho}^{(n+m)}(p \times q) \quad \text { and } \quad \epsilon_{\rho}:=\epsilon_{\rho}^{(2)}\left(\begin{array}{ll}
1 & 2 \\
2 & 1
\end{array}\right)=\epsilon(\rho, \rho) .
$$

In particular, there belongs to any Young tableau $\alpha$ of size $n$ a projection $E_{\rho}^{(\alpha)} \in I\left(\rho^{n}, \rho^{n}\right)$. The task of determining the set of those Young tableaus $\alpha$ for which $E_{\rho}^{(\alpha)} \neq 0$ is usually referred to as the classification of the statistics of (the equivalence class of) $\rho$.

As a symmetry need not exist (nor need it be unique) in a given monoidal $\mathrm{C}^{*}$-category, we want to discuss briefly the following situation in which the existence of a symmetry can be established. It is an abstract version of what happens in quantum field theory. Accordingly, the proof of the ensuing lemma is essentially an argument from [7]. Let us thus assume that there is a symmetric binary relation $\perp$ on the set of objects of $\mathcal{C}$ with the following properties:

1. if $\rho_{1} \perp \rho_{2}$ then $\rho_{1} \rho_{2}=\rho_{2} \rho_{1}$;

2. if $t_{j}: \sigma_{j} \rightarrow \tau_{j}(j=1,2)$ and $\sigma_{1} \perp \sigma_{2}, \tau_{1} \perp \tau_{2}$, then $t_{1 \otimes t_{2}}=t_{2} \otimes t_{1}$;

3. there exist two full monoidal $\mathrm{C}^{*}$-subcategories $\mathcal{C}_{1}, \mathcal{C}_{2} \subset \mathcal{C}$ which are equivalent to $\mathcal{C}$ and such that $\mathcal{C}_{1} \perp \mathcal{C}_{2}$, i.e., such that $\rho_{1} \perp \rho_{2}$ for any pair of objects $\rho_{1} \in \mathcal{C}_{1}, \rho_{2} \in \mathcal{C}_{2}$.

Lemma A.2 If a symmetric binary relation $\perp$ with the stated properties exists, then the monoidal $C^{*}$-category $\mathcal{C}$ possesses a unique symmetry $\epsilon$ which satisfies $\epsilon\left(\rho_{1}, \rho_{2}\right)=\mathbf{1}_{\rho_{1} \rho_{2}}$ if $\rho_{1} \perp \rho_{2}$.

Proof: To define $\epsilon\left(\rho_{1}, \rho_{2}\right)$ for given objects $\rho_{1}, \rho_{2}$, choose some unitaries $u_{j}: \rho_{j} \rightarrow \hat{\rho}_{j}$ whose targets fulfil $\hat{\rho}_{1} \perp \hat{\rho}_{2}$ and let $\epsilon\left(\rho_{1}, \rho_{2}\right):=\left(u_{2} \otimes u_{1}\right)^{*}\left(u_{1} \otimes u_{2}\right)$. (This is possible by 3 , a well-defined unitary by 1 , and by 2 it can be seen to be independent of the choices involved.) Then $\epsilon\left(\rho_{2}, \rho_{1}\right)^{*}=\epsilon\left(\rho_{1}, \rho_{2}\right)$ follows immediately, as well as the triviality of $\epsilon\left(\rho_{1}, \rho_{2}\right)$ in the case that $\rho_{1} \perp \rho_{2}$, and the equation $\epsilon\left(\tau_{1}, \tau_{2}\right) \circ t_{1} \otimes t_{2}=t_{2} \otimes t_{1} \circ \epsilon\left(\sigma_{1}, \sigma_{2}\right)$ (for $t_{j}: \sigma_{j} \rightarrow \tau_{j}$ ) is verified using 2 . The check of the distributional law relies on 3 : Let $\rho_{1}, \rho_{2}, \rho \in \mathcal{C}$ be given. Choose a unitary $u: \rho \rightarrow \hat{\rho}$ such that $\hat{\rho} \in \mathcal{C}_{1}$ above, and choose unitaries $u_{j}: \rho_{j} \rightarrow \tau_{j}$ with $\tau_{j} \in \mathcal{C}_{2}$. Then $\epsilon\left(\rho_{j}, \rho\right)=\left(u \otimes u_{j}\right)^{*}\left(u_{j} \otimes u\right)$, and therefore $\epsilon\left(\rho_{1}, \rho\right) \otimes \mathbf{1}_{\rho_{2}} \circ \mathbf{1}_{\rho_{1}} \otimes \epsilon\left(\rho_{2}, \rho\right)$ can be computed to be $u^{*} \otimes u_{1}^{*} \otimes u_{2}^{*} \circ u_{1} \otimes u_{2} \otimes u$. Now since $u_{1} \otimes u_{2}$ : $\rho_{1} \rho_{2} \rightarrow \tau_{1} \tau_{2} \in \mathcal{C}_{2}$, the last expression equals $\epsilon\left(\rho_{1} \rho_{2}, \rho\right)$, which was the assertion. By choosing $\rho_{1}=\rho_{2}=\iota$ and making use of unitarity, one obtains $\epsilon(\iota, \rho)=\mathbf{1}_{\rho}=\epsilon(\rho, \iota)$, which completes the proof of existence. To see the uniqueness of $\epsilon$, let $\tilde{\epsilon}$ be another symmetry with the property that $\tilde{\epsilon}\left(\tilde{\rho}_{1}, \tilde{\rho}_{2}\right)=1_{\tilde{\rho}_{1} \tilde{\rho}_{2}}=\epsilon\left(\tilde{\rho}_{1}, \tilde{\rho}_{2}\right)$ if $\tilde{\rho}_{1} \perp \tilde{\rho}_{2}$. As one can choose for arbitrary $\rho_{1}, \rho_{2} \in \mathcal{C}$ objects $\hat{\rho}_{1}, \hat{\rho}_{2}$ with $\hat{\rho}_{1} \perp \hat{\rho}_{2}$ and unitaries $u_{j}: \rho_{j} \rightarrow \hat{\rho}_{j}$, it follows that $\tilde{\epsilon}\left(\rho_{1}, \rho_{2}\right)=u_{2}^{*} \otimes u_{1}^{*} \circ \tilde{\epsilon}\left(\hat{\rho}_{1}, \hat{\rho}_{2}\right) \circ u_{1} \otimes u_{2}=u_{2}^{*} \otimes u_{1}^{*} \circ \epsilon\left(\hat{\rho}_{1}, \hat{\rho}_{2}\right) \circ u_{1} \otimes u_{2}=\epsilon\left(\rho_{1}, \rho_{2}\right)$.

\footnotetext{
${ }^{2}$ Here, $p \times q$ is the obvious element of $\mathbb{P}_{n+m}$ associated to $p \in \mathbb{P}_{n}$ and $q \in \mathbb{P}_{m}$ via $\{1, \ldots, n+m\}=$ $\{1, \ldots, n\} \cup\{n+1, \ldots, n+m\}$; cf. Thm. 4.15 of [30]
} 


\section{A.3 Finite Statistics and the Existence of Conjugates}

Let $\mathcal{C}$ be a $\mathrm{rW}^{*}$-category and assume it to be equipped with a symmetry $\epsilon$ and to have left inverses. In this section, we will review the answer to the question under which conditions $\mathcal{C}$ even possesses conjugates and how to obtain them. The solution of this problem is due to Doplicher, Haag and Roberts [7] (cf. also [25]), and it is closely linked to the classification of statistics, which, in turn, is rendered possible by the very presence of left inverses. In view of these results, it is not an overstatement to say that, though being interesting structures on their own, a symmetry and left inverses acquire their real power only through their joint appearance.

To begin with, one identifies in the $\mathrm{rW}^{*}$-category $\mathcal{C}$ certain objects with finite statistics, that is, objects $\rho$ with the property that $\varphi_{\rho, \rho}\left(\epsilon_{\rho}\right) \neq 0$ for all $\varphi \in \operatorname{LI}(\rho)$. We will denote the full subcategory of these objects with $\mathcal{C}_{\text {f.s. }}$. (The remaining objects are said to have infinite statistics.)

A careful analysis then reveals that

1. $\mathcal{C}_{\text {f.s. }}$ is closed under subobjects and finite direct sums; in fact, $\mathcal{C}_{\text {f.s. }}$ consists exactly of those objects of $\mathcal{C}$ which are finite direct sums of irreducible objects with finite statistics;

2. $\mathcal{C}_{\text {f.s. }}$ is closed under the monoidal product;

3. Two additive and multiplicative functions on the objects of $\mathcal{C}_{\text {f.s. }}$,

$$
d: \rho \longmapsto d(\rho) \in \mathbb{N} \quad \text { and } \quad \kappa: \rho \longmapsto \kappa(\rho) \in\left\{t: \rho \rightarrow \rho \mid t^{*}=t, t^{2}=\mathbf{1}_{\rho}\right\},
$$

called the statistical dimension and the statistical phase, respectively, are uniquely defined by the requirement that $\varphi_{\rho, \rho}\left(\epsilon_{\rho}\right)=\frac{\kappa(\rho)}{d(\rho)}$ for any $\rho \in \mathcal{C}_{\text {f.s. }}$ and any "standard" (cf. below) left inverse $\varphi \in \operatorname{LI}(\rho)$.

We will not reproduce the proof of these assertions here, but content ourselves with three brief remarks:

- The $\mathrm{w}^{*}$-compactness of the sets $\operatorname{LI}(\rho)$ (which distinguishes the $\mathrm{W}^{*}$ from the mere $\mathrm{C}^{*}$ situation) enters into the proof of 1 . More precisely, it is used to show that an object of $\mathcal{C}$ which is an infinite direct sum necessarily has infinite statistics. If this conclusion can be drawn from other (more specific) properties, then all of the following remains valid in the $\mathrm{C}^{*}$ case.

- The proof of 2 is remarkably involved as it cannot, actually, be separated from that of 3. A major role in the proof of 2 and 3 is played by the so-called standard left inverses which are defined by the property that $\varphi_{\rho, \rho}\left(\epsilon_{\rho}\right)^{2} \in \mathbb{C} \cdot \mathbf{1}_{\rho}$. Their existence is trivial for irreducible objects and is easily established for reducible ones by a direct sum-like construction.

- Since $\kappa(\rho)$ is self-adjoint and of square $\mathbf{1}_{\rho}$, its spectral decomposition induces a (unique, up to unitary equivalence) decomposition $\rho=\rho_{\mathrm{B}} \oplus \rho_{\mathrm{F}}$ of any object $\rho$ into its bosonic and its fermionic part, where an object $\tau$ is called (purely) bosonic (resp. fermionic) iff $\kappa(\tau)=\mathbf{1}_{\tau}$ (resp. $\kappa(\tau)=-\mathbf{1}_{\tau}$ ). One thus obtains a $\mathbb{Z}_{2}$-grading on the semi-ring underlying $\mathcal{C}_{\text {f.s. }}$. 
The classification of statistics emerges together with the proof of 2 and 3 . Its final result can be expressed in terms of the numbers $b(\rho):=d\left(\rho_{\mathrm{B}}\right)$ and $f(\rho):=d\left(\rho_{\mathrm{F}}\right)$ and says that a Young tableau appears in $\left[\epsilon_{\rho}^{(n)}\right]_{n \in \mathbb{N}}$ iff its $(b(\rho)+1$ )-th row has length $\leq f(\rho)$ (or, equivalently, its $(f(\rho)+1)$-th column has length $\leq b(\rho))$. (Moreover, for $\rho$ with infinite statistics, i.e., $\rho \in \mathcal{C} \backslash \mathcal{C}_{\text {f.s. }}$, there is no restriction on the Young tableaus.)

Eventually, the existence of conjugates can be discussed. It is seen from [37] that an object of $\mathcal{C}$ having a conjugate has finite statistics[3], hence $\mathcal{C}_{\text {f.d. }} \subset \mathcal{C}_{\text {f.s. }}$, and that the dimension function on $\mathcal{C}_{\text {f.d. }}$ coincides with (the restriction of) the statistical dimension $d$ introduced in 3 above. Thus the infinity of the statistics is an obstruction to the existence of a conjugate. One would like this to be the only obstruction, i.e., $\mathcal{C}_{\text {f.d. }}=\mathcal{C}_{\text {f.s. }}$. While this need not be true in general, the important fact in this context is that it is sufficient to consider the simple objects only:

Definition: An object $\gamma \in \mathcal{C}_{\text {f.s. }}$ is called simple if it has statistical dimension $d(\gamma)=1$.

It follows from the algebraic properties of $d$ that the simple objects are irreducible and form a monoid in $\mathcal{C}_{\text {f.s. }}$. In particular, $\epsilon_{\gamma} \in\left\{ \pm \mathbf{1}_{\gamma^{2}}\right\}$ if $\gamma$ is simple. Moreover, if $\gamma$ has a conjugate $\bar{\gamma}$, then $\bar{\gamma}$ is simple too, and it is a monoidal inverse of $\gamma$, i.e., $\bar{\gamma} \cdot \gamma \cong \iota \cong \gamma \cdot \bar{\gamma}$.

The simple objects are of fundamental importance for the following reason:

Proposition A.3 Let all simple objects in $\mathcal{C}_{\text {f.s. }}$ have conjugates. Then every object $\rho \in$ $\mathcal{C}_{\text {f.s. }}$ has a conjugate.

We will not reproduce the complete proof of this proposition here but refer the reader to [25] where a proof in the present categorical language can best be found. As it is rather constructive and relies on almost all of the notions introduced above, we limit ourselves to a few sketchy comments:

- Since a conjugate for a finite direct sum can easily be obtained from the conjugates of its direct summands, it is sufficient to assume that $\rho$ is irreducible.

- Thus let $\rho \in \mathcal{C}_{\text {f.s. }}$ be irreducible with statistical dimension $D:=d(\rho)$. The analysis of the statistics of $\rho$ allows one to define certain subobjects $w_{\gamma}: \gamma \hookrightarrow \rho^{D}$ and $w_{\rho^{\prime}}: \rho^{\prime} \hookrightarrow \rho^{D-1}$. Among other properties, they have the dimensions $d(\gamma)=1$ and $d\left(\rho^{\prime}\right)=D$.

- Since $\gamma \in \mathcal{C}_{\text {f.s. }}$ is simple, by assumption it possesses a conjugate $\bar{\gamma} \in \mathcal{C}_{\text {f.s. }}$. Therefore the object $\bar{\rho}:=\rho^{\prime} \bar{\gamma}$ can be defined. It is easily seen that $\bar{\rho}$ has statistical dimension $D$ and that it is bosonic (resp. fermionic) iff $\rho$ is.

- As suggested by the notation, $\rho$ and $\bar{\rho}$ are conjugate. This is proved by checking that the pair $(r, \bar{r})$ of morphisms given by

$$
\bar{r}:=\sqrt{D}\left(\mathbf{1}_{\rho} \otimes w_{\rho^{\prime}}^{*} \circ w_{\gamma}\right) \otimes \mathbf{1}_{\bar{\gamma}} \circ \bar{u} \quad \text { and } \quad r:=D \varphi_{\iota, \bar{\rho} \rho}\left(\bar{r} \otimes \mathbf{1}_{\rho}\right)
$$

(where $\varphi \in \operatorname{LI}(\rho)$ and $\bar{u}: \iota \rightarrow \gamma \bar{\gamma}$ is a unitary) satisfies the corresponding conjugate equations.

\footnotetext{
${ }^{3}$ The argument is not very difficult: If $\rho$ has a conjugate $\bar{\rho}$ and $(r, \bar{r})$ is a solution of the conjugate equations then every left inverse $\varphi \in \operatorname{LI}(\rho)$ has the form $\varphi_{\sigma, \tau}(t)=r^{*} \otimes \mathbf{1}_{\tau} \circ m \otimes t \circ r \otimes \mathbf{1}_{\sigma}$ for some properly normalised positive $m \in I(\bar{\rho}, \bar{\rho})$. Evaluating this on $\epsilon_{\rho}$ yields $\varphi_{\rho, \rho}\left(\epsilon_{\rho}\right)=\mathbf{1}_{\rho} \otimes r^{*} \circ\left(\epsilon(\bar{\rho}, \rho) \circ m \otimes \mathbf{1}_{\rho} \circ r\right) \otimes \mathbf{1}_{\rho}$ (after some computation). Now this last expression vanishes iff $m$ vanishes, due to the conjugate equations satisfied by $(r, \bar{r})$. But $m=0$ is impossible, hence $\varphi_{\rho, \rho}\left(\epsilon_{\rho}\right) \neq 0$ for each left inverse $\varphi$ of $\rho$.
} 
- In the course of verifying $r^{*} \mathbf{1}_{\bar{\rho}} \circ \mathbf{1}_{\bar{\rho} \otimes \bar{r}}=\mathbf{1}_{\bar{\rho}}$ and $\bar{r}^{*} \otimes \mathbf{1}_{\rho} \circ \mathbf{1}_{\rho} \otimes r=\mathbf{1}_{\rho}$, it is convenient to establish the additional equations

$$
r^{*} r=D=\bar{r}^{*} \bar{r} \quad \text { and } \quad r= \pm \epsilon(\rho, \bar{\rho}) \circ \bar{r}
$$

the sign corresponding to the $\mathbb{Z}_{2}$-grade of $\rho$. More precisely, one can, in a first step, verify the first of the conjugate equations by reducing it to the identity $D^{2} \varphi_{\rho^{D-1}, \rho^{D-1}}\left(w_{\gamma} w_{\gamma}^{*}\right)=w_{\rho^{\prime}} w_{\rho^{\prime}}^{*}$ (which in turn relies on the definition of the objects $\gamma$ and $\rho^{\prime}$ and the representation theory of the permutation groups). In a second step, one can deduce $\bar{r}^{*} \bar{r}=D$ by applying some $\bar{\varphi} \in \operatorname{LI}(\bar{\rho})$ to this first conjugate equation. The rest of (A.1) then follows directly from the definition of $r$. In a third step, one uses the results of the two previous ones in order to show that $y:=\bar{r}^{*}{ }_{\otimes} \mathbf{1}_{\rho} \circ \mathbf{1}_{\rho} \otimes r$ satisfies $y^{*}=y=y^{2}$ and $\varphi_{\iota, \iota}(y)=1$ and infers from this that $y=\mathbf{1}_{\rho}$. That the proof of the two conjugate equations is not symmetric is of course due to the asymmetry in the definition between $r$ and $\bar{r}$.

We mention as an aside that the morphisms $r$ and $\bar{r}$ which identify $(\rho, \bar{\rho})$ as being conjugate to each other, occasionally are required to satisfy the relation $r=\epsilon(\rho, \bar{\rho}) \circ \bar{r}$ in addition to the conjugate equations. While this relation need not be satisfied with a given symmetry, it can always be achieved at the price of replacing that given symmetry with its bosonised version (often denoted with $\hat{\epsilon}$ ), cf. [2.9] for a detailed discussion. Note that $\hat{\epsilon}$ contains less information than $\epsilon$ : since $\varphi_{\rho, \rho}\left(\hat{\epsilon}_{\rho}\right)=\frac{\mathbf{1}_{\rho}}{d(\rho)}$ for any standard left inverse $\varphi \in \operatorname{LI}(\rho)$, the $\mathbb{Z}_{2}$-grading on $\mathcal{C}_{\text {f.s. }}$ is encoded in $\epsilon$, but not in $\hat{\epsilon}$.

As a last remark, we want to point out that - since the definition of objects with finite statistics and that of finite dimensional objects makes reference to other objects in the category under consideration - it may happen that an object $\rho \in \mathcal{C}$ having one of these properties looses that property when regarded as an object $\rho \in \mathcal{C}_{0}$ in some subcategory $\mathcal{C}_{0}$ of $\mathcal{C}$. (And even $\mathcal{C}_{0} \subset \mathcal{C}$ being a full subcategory does not exclude such a phenomenon.) In the relevant situations in the main text, however, such a danger does not occur, since in the inclusions of subcategories which appear there (especially in the "geometric" ones of the type $\Delta(X) \subset \Delta(Y)$ with $X \subset Y$ ), the (full) subcategory $\mathcal{C}_{0} \subset \mathcal{C}$ is usually equivalent to $\mathcal{C}$. Thus the necessary consistency is assured when Prop. A.3 is applied in Section 3.5.

\section{A.4 Standard Solutions and the Conjugation on Morphisms}

We collect here some facts concerning the so-called standard solutions of the conjugate equations and the conjugations $\dagger$ on the morphisms induced by them.

Let $\mathcal{C}$ be an $\mathrm{rC}^{*}$-category and let $\mathcal{C}_{\text {f.d. }}$ be the full subcategory of finite dimensional objects. Recall that the latter are, by definition, those objects $\rho$ which possess a conjugate $\bar{\rho}$ and that we denote with $\mathcal{R}(\rho, \bar{\rho})$ the set of all solutions of the conjugate equations. Thus, $(r, \bar{r}) \in \mathcal{R}(\rho, \bar{\rho})$ means that the morphisms $r: \iota \rightarrow \bar{\rho} \rho$ and $\bar{r}: \iota \rightarrow \rho \bar{\rho}$ satisfy

$$
\bar{r}^{*} \otimes \mathbf{1}_{\rho} \circ \mathbf{1}_{\rho} \otimes r=\mathbf{1}_{\rho} \quad \text { and } \quad r^{*} \mathbf{1}_{\bar{\rho}} \circ \mathbf{1}_{\bar{\rho} \otimes \bar{r}}=\mathbf{1}_{\bar{\rho}} .
$$

Such a solution is said to be normalised if $\|r\|=\|\bar{r}\|$.

The set $\mathcal{R}(\rho, \bar{\rho})$ may also be described as follows: 
Lemma A.4 The group $\operatorname{GL}(\rho):=\{a \in I(\rho, \rho) \mid a$ is invertible $\}$ acts on $\mathcal{R}(\rho, \bar{\rho})$ according to

$$
a \star(r, \bar{r}):=\left(\mathbf{1}_{\bar{\rho}}^{\otimes a} \circ r, a^{*-1} \otimes \mathbf{1}_{\bar{\rho}} \circ \bar{r}\right) .
$$

This action is ergodic and free, i.e., any element $\left(r^{\prime}, \bar{r}^{\prime}\right)$ of $\mathcal{R}(\rho, \bar{\rho})$ can be obtained from any other element $(r, \bar{r})$ in a unique way: for $a \in \operatorname{GL}(\rho)$, the relation $\left(r^{\prime}, \bar{r}^{\prime}\right)=a \star(r, \bar{r})$ is equivalent to either of

$$
a=\bar{r}^{*} \otimes \mathbf{1}_{\rho} \circ \mathbf{1}_{\rho} \otimes r^{\prime}, \quad a^{-1}=\bar{r}^{* *} \otimes \mathbf{1}_{\rho} \circ \mathbf{1}_{\rho} \otimes r .
$$

The straightforward verification of these assertions is left as an exercise to the reader.

As has already been mentioned in Appendix A.2, $\mathcal{C}_{\text {f.d. }}$ consists of all finite direct sums of irreducible objects and is closed under the monoidal product. The two semiring operations $\oplus$ and $\cdot$ on the objects of $\mathcal{C}_{\text {f.d. }}$ have their counterparts on the solutions of the conjugate equations:

- Let $\left(r_{1}, \bar{r}_{1}\right) \in \mathcal{R}\left(\rho_{1}, \bar{\rho}_{1}\right)$ and $\left(r_{2}, \bar{r}_{2}\right) \in \mathcal{R}\left(\rho_{2}, \bar{\rho}_{2}\right)$. Then $\left(r_{12}, \bar{r}_{12}\right) \in \mathcal{R}\left(\rho_{1} \rho_{2}, \bar{\rho}_{2} \bar{\rho}_{1}\right)$, where

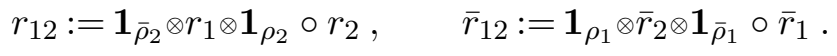

The pair $\left(r_{12}, \bar{r}_{12}\right)$ is called the product of $\left(r_{1}, \bar{r}_{1}\right)$ and $\left(r_{2}, \bar{r}_{2}\right)$.

- Let $\left(r_{j}, \bar{r}_{j}\right) \in \mathcal{R}\left(\rho_{j}, \bar{\rho}_{j}\right)$ (with $\left.j=1, \ldots, J\right)$. Let $\rho=\bigoplus \rho_{j}$ (resp. $\bar{\rho}=\bigoplus \bar{\rho}_{j}$ ) be the direct sums of the families of objects and $w_{j}: \rho_{j} \hookrightarrow \rho\left(\right.$ resp. $\left.\bar{w}_{j}: \bar{\rho}_{j} \hookrightarrow \bar{\rho}\right)$ the corresponding families of pairwise orthogonal isometries. Then $(r, \bar{r}) \in \mathcal{R}(\rho, \bar{\rho})$, where

$$
r:=\sum_{j} \bar{w}_{j} \otimes w_{j} \circ r_{j}, \quad \bar{r}:=\sum_{j} w_{j} \otimes \bar{w}_{j} \circ \bar{r}_{j} .
$$

The pair $(r, \bar{r})$ is called the direct sum (via the families $\left(w_{j}\right)$ and $\left(\bar{w}_{j}\right)$ ) of the solutions $\left(r_{j}, \bar{r}_{j}\right)$.

We notice for later use that the expressions $r^{*} r$ and $\bar{r}^{*} \bar{r}$ (which actually just are positive numbers in $I(\iota, \iota)=\mathbb{C}$ ) are well-behaved under these two operations: with the above notations, one has

$$
r_{12}^{*} r_{12}=r_{1}^{*} r_{1} r_{2}^{*} r_{2}, \quad \bar{r}_{12}^{*} \bar{r}_{12}=\bar{r}_{1}^{*} \bar{r}_{1} \bar{r}_{2}^{*} \bar{r}_{2}
$$

and

$$
r^{*} r=\sum_{j} r_{j}^{*} r_{j}, \quad \bar{r}^{*} \bar{r}=\sum_{j} \bar{r}_{j}^{*} \bar{r}_{j}
$$

As a particular consequence, products and direct sums of normalised solutions are normalised.

A very important class of solutions of the conjugate equations is that of standard solutions. Among the numerous equivalent ways in which they can be introduced, the following is suited for our purposes:

Definition: Let $(r, \bar{r}) \in \mathcal{R}(\rho, \bar{\rho})$. Then $(r, \bar{r})$ is called a standard solution if it is the direct sum (via suitable families of pairwise orthogonal isometries) of a family $\left(r_{j}, \bar{r}_{j}\right) \in$ $\mathcal{R}\left(\rho_{j}, \bar{\rho}_{j}\right)(j=1, \ldots, J)$ of normalised solutions, where the objects $\rho_{j}$ and $\bar{\rho}_{j}$ are irreducible, conjugate to each other, and such that $\rho \cong \bigoplus_{j} \rho_{j}$ and $\bar{\rho} \cong \bigoplus_{j} \bar{\rho}_{j}$. 
Appendix A. Some Category Theory

It is obvious from this definition that standard solutions are normalised and that the direct sum of standard solutions is standard again. It is much less obvious but nevertheless true that the class of standard solutions is also closed under the product; i.e., the product of two standard solutions is again standard. We refer the reader to [37, 40] for the proof of this statement, in the course of which several other properties and characterisations of standard solutions are derived. Let us just quote two of these characterisations (whose mutual equivalence is easily seen if it is taken into account that $r^{*} r=\|r\|^{2}$ and $\left.\bar{r}^{*} \bar{r}=\|\bar{r}\|^{2}\right)$ :

- Standard solutions are those normalised elements of $\mathcal{R}(\rho, \bar{\rho})$ for which the value of the function $(r, \bar{r}) \longmapsto\|r\|\|\bar{r}\|$ is minimal. (This minimal value is, by definition, the dimension $d(\rho)$.)

- $(r, \bar{r}) \in \mathcal{R}(\rho, \bar{\rho})$ is standard iff $r^{*} r=d(\rho)=\bar{r}^{*} \bar{r}$.

Because of the relations (A.2) and (A.3) this last characterisation shows that the additivity resp. multiplicativity of the dimension is equivalent to the closedness of the class of standard solutions under direct sums resp. products. (In view of the proof in [37], it would be cheating to replace the words "is equivalent to" with "proves"!)

As a last remark on the above definition, we notice that as far as irreducible objects $\sigma$ are concerned, there is no distinction between normalised and standard solutions, and that all standard solutions $(r, \bar{r}) \in \mathcal{R}(\sigma, \bar{\sigma})$ are equal up to a phase (cf. Lemma A.4). Correspondingly, it will not come as a surprise that the analogous freedom in the case of reducible objects $\rho$ only amounts to the multiplication with a unitary (cf. Lemma A.6 below).

In the main text (and for the proof of Lemma A.6) we need the notion of conjugation on morphisms:

Definition: Let $(r, \bar{r}) \in \mathcal{R}(\rho, \bar{\rho})$ be a solution of the conjugate equations. The two antilinear maps

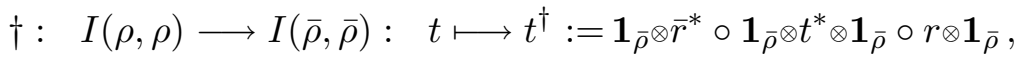

$$
\begin{aligned}
& \dagger: \quad I(\bar{\rho}, \bar{\rho}) \longrightarrow I(\rho, \rho): \bar{t} \longmapsto \bar{t}^{\dagger}:=\mathbf{1}_{\rho} \otimes r^{*} \circ \mathbf{1}_{\rho} \otimes \bar{t}^{*}{ }_{\otimes} \mathbf{1}_{\rho} \circ \bar{r} \otimes \mathbf{1}_{\rho}
\end{aligned}
$$

are called the conjugation induced by $(r, \bar{r})$.

One directly verifies the relations

$$
t^{\dagger \dagger}=t, \quad \mathbf{1}_{\rho}^{\dagger}=\mathbf{1}_{\bar{\rho}}, \quad(t \circ s)^{\dagger}=t^{\dagger} \circ s^{\dagger} .
$$

Another identity which one would tend to expect is the equality of $t^{\dagger^{*}}$ and $t^{* \dagger}$, but such a relation does not hold in general. For standard solutions, however, it does (but not only for standard solutions, as will be noticed after Lemma A.6):

Lemma A.5 Let $(r, \bar{r}) \in \mathcal{R}(\rho, \bar{\rho})$ and $\dagger$ be as above. If $(r, \bar{r})$ is standard, then $t^{\dagger^{*}}=t^{* \dagger}$ holds for all $t \in I(\rho, \rho)$.

Proof: By our definition of standard solutions, there exist pairs $\left(\rho_{j}, \bar{\rho}_{j}\right)$ of irreducible objects conjugate to each other, normalised solutions $\left(r_{j}, \bar{r}_{j}\right) \in \mathcal{R}\left(\rho_{j}, \bar{\rho}_{j}\right)$ and families of pairwise orthogonal isometries $w_{j}: \rho_{j} \hookrightarrow \rho\left(\right.$ resp. $\left.\bar{w}_{j}: \bar{\rho}_{j} \hookrightarrow \bar{\rho}\right)$ performing a decomposition $\rho=\bigoplus_{j} \rho_{j}$ (resp. $\left.\bar{\rho}=\bigoplus_{j} \bar{\rho}_{j}\right)$ such that $(r, \bar{r}) \in \mathcal{R}(\rho, \bar{\rho})$ is the direct sum of the family $\left(r_{j}, \bar{r}_{j}\right)$. It follows from the definition of $\dagger$ that one has for any $k, l$ :

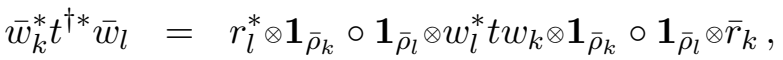

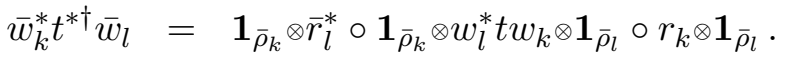


It is sufficient (and necessary) that the right-hand sides coincide. This is trivially the case when $\rho_{k}$ and $\rho_{l}$ are disjoint, since then $w_{l}^{*} t w_{k}=0$. In the other case, $\rho_{k}$ and $\rho_{l}$ are equivalent, and the same is true for $\bar{\rho}_{k}$ and $\bar{\rho}_{l}$. One may thus choose unitaries $u \in I\left(\rho_{k}, \rho_{l}\right)$ and $\bar{u} \in I\left(\bar{\rho}_{k}, \bar{\rho}_{l}\right)$ serving as basis elements in these 1-dimensional vector spaces. In particular, $w_{l}^{*} t w_{k}=T u$ for some complex number $T$. Moreover, one can use $u$ and $\bar{u}$ to turn $\left(r_{k}, \bar{r}_{k}\right)$ into a solution $\left(r_{l}^{\prime}, \bar{r}_{l}^{\prime}\right) \in \mathcal{R}\left(\rho_{l}, \bar{\rho}_{l}\right)$ by setting $r_{l}^{\prime}:=\bar{u} \otimes u \circ r_{k}$ and $\bar{r}_{l}^{\prime}:=u \otimes \bar{u} \circ \bar{r}_{k}$. A direct computation shows that

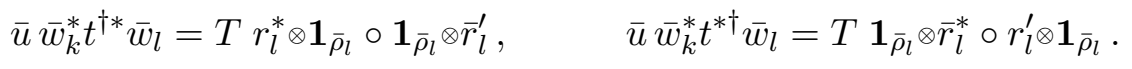

But since $\rho_{l}$ is irreducible and the solutions $\left(r_{l}^{\prime}, \bar{r}_{l}^{\prime}\right)$ and $\left(r_{l}, \bar{r}_{l}\right)$ are normalised, they differ only by a phase: $\left(r_{l}^{\prime}, \bar{r}_{l}^{\prime}\right)=\left(e^{i \xi} \mathbf{1}_{\rho_{l}}\right) \star\left(r_{l}, \bar{r}_{l}\right)=\left(e^{i \xi} r_{l}, e^{i \xi} \bar{r}_{l}\right), \xi \in \mathbb{R}$. Hence,

$$
\bar{u} \bar{w}_{k}^{*} t^{\dagger *} \bar{w}_{l}=T e^{i \xi} \mathbf{1}_{\bar{\rho}_{l}}=\bar{u} \bar{w}_{k}^{*} t^{* \dagger} \bar{w}_{l},
$$

which completes the proof.

Lemma A.5 guarantees that $\dagger$ maps self-adjoint (resp. unitary) elements to self-adjoint (resp. unitary) ones. It is also used in the proof of the next result, whose "only if" part may be interpreted as saying that the standard solutions are "as unique as possible" (in view of their characterisation by the minimality of $\|r\|\|\bar{r}\|$ and their nature as morphisms between different objects in a $\mathrm{C}^{*}$-category).

Lemma A.6 If $(r, \bar{r}) \in \mathcal{R}(\rho, \bar{\rho})$ be a standard solution and let $a \in \operatorname{GL}(\rho)$. Then $a \star(r, \bar{r})$ is standard iff a is unitary.

Proof: The "if" part is trivial since the unitary a can always be absorbed into the isometries $w_{j}: \rho_{j} \hookrightarrow \rho$ which perform a decomposition of $\rho$ into irreducibles. For the proof of the "only if" part, let us write $\left(r_{1}, \bar{r}_{1}\right):=(r, \bar{r})$ and $\left(r_{2}, \bar{r}_{2}\right):=a \star(r, \bar{r})$. According to Lemma A.4, $a \in \mathrm{GL}(\rho)$ satisfies $a=\bar{r}_{1}^{*} \mathbf{1}_{\rho} \circ \mathbf{1}_{\rho} \otimes r_{2}$ and $a^{-1}=\bar{r}_{2}^{*} \mathbf{1}_{\rho} \circ \mathbf{1}_{\rho} \otimes r_{1}$. We have to use the standard property of both $\left(r_{1}, \bar{r}_{1}\right)$ and $\left(r_{2}, \bar{r}_{2}\right)$ in order to show $a^{*}=a^{-1}$. To this end, we consider the direct sums $\sigma:=\rho \oplus \rho$ and $\bar{\sigma}:=\bar{\rho} \oplus \bar{\rho}$. We have two pairs $w_{1}, w_{2}$ and $\bar{w}_{1}, \bar{w}_{2}$ of isometries subject to the usual orthogonality and completeness relations. Let $\dagger$ denote the conjugation between $I(\sigma, \sigma)$ and $I(\bar{\sigma}, \bar{\sigma})$ induced by the direct sum of $\left(r_{1}, \bar{r}_{1}\right)$ and $\left(r_{2}, \bar{r}_{2}\right)$. Setting $t:=\bar{w}_{2} \bar{w}_{1}^{*} \in I(\bar{\sigma}, \bar{\sigma})$, one now verifies the identities

$$
w_{1}^{*} t^{* \dagger} w_{2}=\mathbf{1}_{\rho} \otimes r_{2}^{*} \circ \bar{r}_{1 \otimes} \mathbf{1}_{\rho}=a^{*}, \quad w_{1}^{*} t^{\dagger *} w_{2}=\bar{r}_{2}^{*} \otimes \mathbf{1}_{\rho} \circ \mathbf{1}_{\rho} \otimes r_{1}=a^{-1} .
$$

Now if $\left(r_{1}, \bar{r}_{1}\right)$ and $\left(r_{2}, \bar{r}_{2}\right)$ are standard, then their direct sum is standard too. By Lemma A.5, this implies $t^{* \dagger}=t^{\dagger *}$, whence $a^{*}=a^{-1}$.

Remark: It is instructive to compare the conjugations associated to different and not necessarily standard solutions of the conjugate equations for $(\rho, \bar{\rho})$. Let $(r, \bar{r})$ and $\left(r^{\prime}, \bar{r}^{\prime}\right)$ be related by $\left(r^{\prime}, \bar{r}^{\prime}\right)=a \star(r, \bar{r})$ and denote the respective conjugations with $\dagger$ and $\dagger^{\prime}$. They are easily found to fulfil $t^{\dagger^{\prime}}=a^{* \dagger} t^{\dagger}\left(a^{* \dagger}\right)^{-1}$ for all $t \in I(\rho, \rho)$.

In the case when $(r, \bar{r})$ is standard, one obtains from this (with the abbreviation $\left.\bar{a}:=a^{* \dagger}=a^{\dagger *}\right)$

$$
t^{* \dagger^{\prime}}=\bar{a} t^{* \dagger} \bar{a}^{-1}, \quad t^{\dagger^{*} *}=\left(\bar{a}^{*}\right)^{-1} t^{\dagger *} \bar{a}^{*}
$$


and because of $t^{* \dagger}=t^{\dagger *}$, it follows that for each $t \in I(\rho, \rho)$

$$
t^{* \dagger^{\prime}}=t^{\dagger^{\prime} *} \quad \Longleftrightarrow \quad \bar{a}^{*} \bar{a} t^{* \dagger}=t^{* \dagger} \bar{a}^{*} \bar{a} \quad \Longleftrightarrow a a^{*} t=t a a^{*} .
$$

Thus the identity $* \dagger^{\prime}=\dagger^{\prime} *$ holds iff $a a^{*}$ lies in the centre of the $\mathrm{C}^{*}$-algebra $I(\rho, \rho)$. Hence, unless the latter is a factor, there exist (normalised) solutions $\left(r^{\prime}, \bar{r}^{\prime}\right) \in \mathcal{R}(\rho, \bar{\rho})$ which are not standard but still fulfil $* \dagger^{\prime}=\dagger^{\prime} *$. In particular, the property $* \dagger=\dagger *$ does not characterise standard solutions. As a matter of fact, it can also be shown that it is conserved neither under products nor under direct sums.

Until now we have only considered the conjugation between the spaces $I(\rho, \rho)$ and $I(\bar{\rho}, \bar{\rho})$ of morphisms for fixed objects $\rho$ and $\bar{\rho}$. In many computations however (especially in Section 3.7), one needs the conjugation between pairs of spaces like $I(\sigma, \tau)$ and $I(\bar{\sigma}, \bar{\tau})$. Each such conjugation is given by a pair of solutions $\left(r_{\sigma}, \bar{r}_{\sigma}\right) \in \mathcal{R}(\sigma, \bar{\sigma})$ and $\left(r_{\tau}, \bar{r}_{\tau}\right) \in$ $\mathcal{R}(\tau, \bar{\tau})$ of the conjugate equations. In order to ensure that these conjugations have the expected behaviour under composition, the ${ }^{*}$-operation and under monoidal products, it must be ensured that the solutions $(r, \bar{r})$ involved match each other. Although this could be done in a more formal manner

Definition: Let $\left(r_{\sigma}, \bar{r}_{\sigma}\right) \in \mathcal{R}(\sigma, \bar{\sigma})$ and $\left(r_{\tau}, \bar{r}_{\tau}\right) \in \mathcal{R}(\tau, \bar{\tau})$ be standard solutions. Then the two antilinear maps

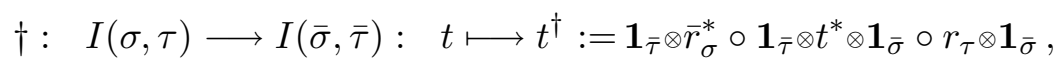

$$
\begin{aligned}
& \dagger: I(\bar{\sigma}, \bar{\tau}) \longrightarrow I(\sigma, \tau): \bar{t} \longmapsto \bar{t}^{\dagger}:=\mathbf{1}_{\tau} \otimes r_{\sigma}^{*} \circ \mathbf{1}_{\tau} \otimes \bar{t}^{*} \otimes \mathbf{1}_{\sigma} \circ \bar{r}_{\tau} \otimes \mathbf{1}_{\sigma}
\end{aligned}
$$

are called the conjugation induced by $\left(r_{\sigma}, \bar{r}_{\sigma}\right)$ and $\left(r_{\tau}, \bar{r}_{\tau}\right)$.

By computations analogous to the case discussed above, one then checks the following:

- The two maps $\dagger$ are inverse to each other, $t^{\dagger \dagger}=t$.

- The morphisms $t: \sigma \rightarrow \tau$ and $t^{\dagger}: \bar{\sigma} \rightarrow \bar{\tau}$ fulfil the following identity (determining them uniquely one from another):

$$
t^{\dagger} \otimes \mathbf{1}_{\sigma} \circ r_{\sigma}=\mathbf{1}_{\bar{\tau} \otimes t^{*} \circ r_{\tau}}
$$

- Let $\left(r_{\rho}, \bar{r}_{\rho}\right) \in \mathcal{R}(\rho, \bar{\rho}),\left(r_{\sigma}, \bar{r}_{\sigma}\right) \in \mathcal{R}(\sigma, \bar{\sigma})$ and $\left(r_{\tau}, \bar{r}_{\tau}\right) \in \mathcal{R}(\tau, \bar{\tau})$ be fixed. Then one has

$$
(t \circ s)^{\dagger}=t^{\dagger} \circ s^{\dagger}
$$

for all $s: \rho \rightarrow \sigma$ and $t: \sigma \rightarrow \tau$ if each of the three conjugations in this formula is induced by the obvious pair of solutions.

- Let $\left(r_{\sigma_{j}}, \bar{r}_{\sigma_{j}}\right) \in \mathcal{R}\left(\sigma_{j}, \bar{\sigma}_{j}\right)$ and $\left(r_{\tau_{j}}, \bar{r}_{\tau_{j}}\right) \in \mathcal{R}\left(\tau_{j}, \bar{\tau}_{j}\right)$ be fixed $(j=1,2)$. Then one has

$$
\left(t_{1} \otimes t_{2}\right)^{\dagger}=t_{2}^{\dagger} \otimes t_{1}^{\dagger}
$$

for all $t_{j}: \sigma_{j} \rightarrow \tau_{j},(j=1,2)$ if the conjugations on the right-hand side are induced by the obvious pairs of solutions and the one on the left-hand side is induced by the obvious pair of products of solutions.

\footnotetext{
${ }^{4}$ The naive approach which supposes that for each object $\rho$ some conjugate $\bar{\rho}$ and a solution $\left(r_{\rho}, \bar{r}_{\rho}\right) \in$ $\mathcal{R}(\rho, \bar{\rho})$ have been fixed once and for all will in general run into inconsistencies. A starting point for a rigorous approach seems to consist in considering a certain (monoidal $\mathrm{C}^{*}$ ) category whose objects are all quadruples $\left(\begin{array}{c}\rho \\ r\end{array} \overline{\bar{r}}\right)$ such that $(r, \bar{r}) \in \mathcal{R}(\rho, \bar{\rho})$ and a "projection" functor from that to $\mathcal{C}$. We refrain from spelling out the details.
} 
- Let $\dagger^{\prime}$ denote the conjugation induced by $a_{\sigma} \star\left(r_{\sigma}, \bar{r}_{\sigma}\right)$ and $a_{\tau} \star\left(r_{\tau}, \bar{r}_{\tau}\right)$, where the morphisms $a_{\sigma}: \sigma \rightarrow \sigma$ and $a_{\tau}: \tau \rightarrow \tau$ are invertible. Then one has for any $t \in I(\sigma, \tau)$

$$
t^{\dagger^{\prime}}=a_{\tau}^{* \dagger} \circ t^{\dagger} \circ\left(a_{\sigma}^{* \dagger}\right)^{-1}
$$

The assumption that the solutions are standard did not yet enter the discussion since it is only important for the relation between the conjugation $\nmid$ and the involution $*$. Things seem slightly more complicated now, because (after exchanging the roles of $\sigma$ and $\tau$ ) the solutions $\left(r_{\tau}, \bar{r}_{\tau}\right)$ and $\left(r_{\sigma}, \bar{r}_{\sigma}\right)$ induce two more conjugations $\dagger^{*}$, namely between the spaces $I(\tau, \sigma)$ and $I(\bar{\tau}, \bar{\sigma})$. A priori, $\dagger^{*}$ is different from the map $* \dagger *$ (which also goes between these two spaces), but since the solutions involved are standard, $\dagger^{*}$ and $* \dagger *$ actually coincide. (This may be seen by a direct sum argument like in the proof of Lemma A.5: the sum of $\left(r_{\sigma}, \bar{r}_{\sigma}\right)$ and $\left(r_{\tau}, \bar{r}_{\tau}\right)$ is a standard solution for $(\rho, \bar{\rho}):=(\sigma \oplus \tau, \bar{\sigma} \oplus \bar{\tau})$, and the conjugation $\ddagger$ between $I(\rho, \rho)$ and $I(\bar{\rho}, \bar{\rho})$ induced by it fulfils $\ddagger *=* \ddagger$. Since by construction $\ddagger$ reproduces $\dagger$ and $\dagger^{*}$ on the respective subspaces, this implies $\dagger^{*} *=* \dagger$, i.e. $\dagger^{*}=* \dagger *$.)

One can thus write $\dagger$ for $\dagger^{*}$ and obtains the formula $t^{\dagger *}=t^{* \dagger}$, which is to be read as a shorthand for saying that the following diagram of antilinear involutive maps commutes:

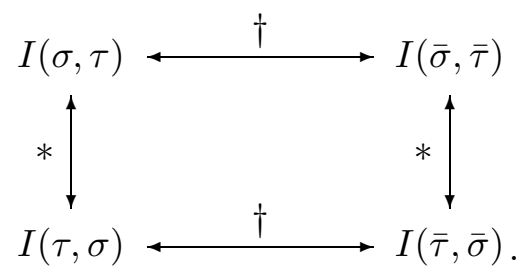





\section{Appendix B}

\section{A Homotopy Argument}

We give here the precise homotopy argument showing that charge transport cocycles on a simply connected group are determined uniquely by their values in a neighbourhood of the unit element. The setting is that of Section 3.7: $\tilde{G}$ is a simply connected Lie group and $\Delta$ is a $\mathrm{W}^{*}$-category carrying an action $\beta$ of $\tilde{G}$. Moreover in Part ii of the following lemma, Mor $\Delta$ is understood to be equipped with an "overall $\mathrm{w}^{*}$-topology" (i.e., with some topology which reproduces the $\mathrm{w}^{*}$-topology on each Banach space $I(\sigma, \tau)$ of morphisms as a subspace topology) with the property that the product of two (pointwise composable) continuous unitary Mor $\Delta$-valued functions is again continuous. The overall $\mathrm{w}^{*}$-topology induced by the map Mor $\Delta \longrightarrow \mathcal{B}(\mathcal{H}): t \longmapsto t$ obviously is of this kind.

\section{Lemma B.1}

i. Let $\rho \in \Delta$ and let $K \subset \tilde{G}$ be a connected neighbourhood of the unit element $\mathbf{1} \in \tilde{G}$. Let the function $\Gamma: K \longrightarrow$ Mor $\Delta$ have the following properties:

- for each $g \in K, \Gamma(g)$ is a unitary from $\rho_{g}$ to $\rho$;

- $\Gamma(g) \circ \beta_{g}\left(\Gamma\left(g^{\prime}\right)\right)=\Gamma\left(g g^{\prime}\right)$ if $\left\{g, g^{\prime}, g g^{\prime}\right\} \subset K$.

Then $\Gamma$ extends uniquely to a function $\Gamma: \tilde{G} \longrightarrow$ Mor $\Delta$ with the same two properties for arbitrary $g, g^{\prime} \in \tilde{G}$.

ii. If in addition $\Gamma: K \longrightarrow$ Mor $\Delta$ and each function $g \longmapsto \beta_{g}(t), t \in$ Mor $\Delta$ are continuous, then the extension $\Gamma: \tilde{G} \longrightarrow \operatorname{Mor} \Delta$ is continuous.

Proof: i. Let $g \in \tilde{G}$ be given. Then there exists (by a compactness argument) a sequence ${ }^{1}$ $g_{0}, g_{1}, \ldots, g_{J} \in \tilde{G}$ with $g_{0}=\mathbf{1}$ and $g_{J}=g$ such that $\delta_{j}:=g_{j-1}^{-1} g_{j} \in K$ for $j=1, \ldots, J$. Define a unitary $\Gamma(g) \in I\left(\rho_{g}, \rho\right)$ by

$$
\Gamma(g):=\Gamma\left(\delta_{1}\right) \circ \beta_{g_{1}}\left(\Gamma\left(\delta_{2}\right)\right) \circ \cdots \circ \beta_{g_{J-1}}\left(\Gamma\left(\delta_{J}\right)\right) .
$$

This expression exists since $\beta_{g_{j-1}}\left(\Gamma\left(\delta_{j}\right)\right) \in I\left(\beta_{g_{j-1}}\left(\beta_{\delta_{j}}(\rho)\right), \beta_{g_{j-1}}(\rho)\right)=I\left(\beta_{g_{j}}(\rho), \beta_{g_{j-1}}(\rho)\right)$, and it has to be shown that it depends on the product $g=\delta_{1} \cdots \delta_{J}$ only. Assuming without restriction that $K$ is closed under taking inverses, one has $\beta_{g^{\prime}}(\Gamma(\delta))^{*}=\beta_{g^{\prime} \delta}\left(\Gamma\left(\delta^{-1}\right)\right)$ for all $g^{\prime} \in \tilde{G}$ and $\delta \in K$. It is therefore sufficient to show that

$P\left(g_{1}, \ldots, g_{J}\right):=\Gamma\left(g_{1}\right) \circ \beta_{g_{1}}\left(\Gamma\left(g_{1}^{-1} g_{2}\right)\right) \circ \cdots \circ \beta_{g_{J-1}}\left(\Gamma\left(g_{J-1}^{-1} g_{J}\right)\right)=\mathbf{1}_{\rho} \quad$ whenever $\quad g_{J}=\mathbf{1}$.

\footnotetext{
${ }^{1}$ Intuitively, this sequence is of course to be regarded as a "discrete path consisting of steps in $K$ ". In the sequel, such paths will be composed and deformed.
} 
Thus assume that $g_{J}=\mathbf{1}$. Then there is a continuous loop $\gamma_{1}:[0,1] \longrightarrow \tilde{G}$ (based at $\mathbf{1} \in \tilde{G})$ and there exist real numbers $0=t_{0} \leq t_{1} \leq \cdots \leq t_{J}=1$ such that $\gamma_{1}\left(t_{j}\right)=g_{j}$ and $\gamma_{1}\left(\left[t_{j}, t_{j+1}\right]\right) \subset g_{j} K(j=0, \ldots, J-1)$. Since $\tilde{G}$ is simply connected, there exists a contracting homotopy $\gamma:[0,1] \times[0,1] \longrightarrow \tilde{G}$ from $\gamma_{1} \equiv \gamma(1, \cdot)$ to the trivial loop $\gamma_{0} \equiv \gamma(0, \cdot)=\mathbf{1}$. A compactness argument now shows that (possibly after changing $J$ into some $J^{\prime} \geq J$ and setting $g_{J+1}=\ldots=g_{J^{\prime}}=1$ ) one can choose numbers $0=s_{0} \leq s_{1} \leq$ $\cdots \leq s_{N}=1$ in such a way that $\gamma(s, t) \in \gamma\left(s_{n}, t_{j}\right) K$ for all $s \in\left[s_{n}, s_{n+1}\right], t \in\left[t_{j}, t_{j+1}\right]$. Setting $g_{n, j}:=\gamma\left(s_{n}, t_{j}\right)$ and $P_{n, j}:=P\left(g_{n, 1}, \ldots, g_{n, j}, g_{n-1, j+1}, \ldots g_{n-1, J}\right)$, one has

$$
\begin{aligned}
P_{0, J} & =P\left(g_{0,1}, \ldots, g_{0, J}\right)=\mathbf{1}_{\rho}, \\
P_{n, 0} & =P\left(g_{n-1,1}, \ldots, g_{n-1, J}\right)=P_{n-1, J}, \quad(n=1, \ldots, N), \\
P_{N, J} & =P\left(g_{N, 1}, \ldots, g_{N, J}\right)=P\left(g_{1}, \ldots, g_{J}\right) .
\end{aligned}
$$

Moreover, $P_{n, j-1}$ and $P_{n, j}$ only differ in their $j$ th and $(j+1)$ th factors:

$$
\begin{aligned}
P_{n, j-1} & =\cdots \circ \beta_{g_{n, j-1}}\left(\Gamma\left(g_{n, j-1}^{-1} g_{n-1, j}\right)\right) \circ \beta_{g_{n-1, j}}\left(\Gamma\left(g_{n-1, j}^{-1} g_{n-1, j+1}\right)\right) \circ \cdots, \\
P_{n, j} & =\cdots \circ \beta_{g_{n, j-1}}\left(\Gamma\left(g_{n, j-1}^{-1} g_{n, j}\right)\right) \circ \beta_{g_{n, j}}\left(\Gamma\left(g_{n, j}^{-1} g_{n-1, j+1}\right)\right) \circ \cdots .
\end{aligned}
$$

By the cocycle equation in $K$, the product of these factors equals $\beta_{g_{n, j-1}}\left(\Gamma\left(g_{n, j-1}^{-1} g_{n-1, j+1}\right)\right)$ in both cases, whence it follows that $P_{n, j-1}=P_{n, j}($ for $j=1, \ldots, J$ and $n=1, \ldots, N)$. An induction yields $P_{n, j}=\mathbf{1}_{\rho}$ for all $n, j$ and thus $P\left(g_{1}, \ldots, g_{J}\right)=\mathbf{1}_{\rho}$. This completes the proof that $\Gamma(g)$ is well-defined. With this result, the validity of $\Gamma(g) \circ \beta_{g}\left(\Gamma\left(g^{\prime}\right)\right)=\Gamma\left(g g^{\prime}\right)$ for arbitrary $g, g^{\prime} \in \tilde{G}$ is an immediate consequence of the above definition.

ii. To see the continuity of $\Gamma$ on $\tilde{G}$, pick some $\tilde{g} \in \tilde{G}$, set $\delta:=g \tilde{g}^{-1}$ and write the cocycle equation as $\Gamma(g)=\Gamma(\delta) \circ \beta_{\delta}(\Gamma(\tilde{g}))$. Notice that $\delta \in K$ iff $g \in K \tilde{g}$. By assumption, $\delta \longmapsto \Gamma(\delta)$ and $\delta \longmapsto \beta_{\delta}(t)$ (for any fixed $t$ ) are continuous on $K$. Taking into account that both $\Gamma(\delta)$ and $\beta_{\delta}(\Gamma(\tilde{g}))$ are unitary, it follows that their product is continuous as a function of $\delta$. Hence $g \longmapsto \Gamma(g)$ is continuous in the neighbourhood $K \tilde{g}$ of $\tilde{g}$. Since $\tilde{g}$ was arbitrary, this proves the continuity of $G$. 


\section{Appendix $\mathrm{C}$}

\section{Proof of Formula (4.4)}

We want to prove in this appendix the formula

$$
t_{1}^{*} V(x) t_{2} U_{I}(x)^{*}=\kappa U_{I}(x)\left(\bar{\rho}^{Y}\left(t_{2}^{*}\right) r\right)^{*} \bar{V}(x)^{*}\left(\bar{\rho}^{Y}\left(t_{1}^{*}\right) r\right) \quad \text { for every } x \in \bar{W}
$$

which is of crucial importance in Section 4.4, where the notation is explained.

This formula has been derived in the so-called field bundle formalism; namely in [8] for the case of compactly localised charges and (with the necessary technical changes regarding the field bundle) in [9] for the case of localisation in spacelike cones.

Here we give a slightly different proof in the language of symmetric monoidal $\mathrm{C}^{*}$ categories. It postpones the contact with $\left(\mathcal{H}_{I}, U_{I}\right)$ and with the specific situation of Section 4.4 until the very end. Accordingly, it is sufficient to assume the following for the time being:

- $\rho, \bar{\rho} \in \Delta(Y)_{\mathrm{f}, \mathrm{c}}$ are conjugate to each other with $(r, \bar{r}) \in \mathcal{R}(\rho, \bar{\rho})$ a standard solution of the conjugate equations.

- $t_{j}: \sigma_{j} \rightarrow \rho$ are morphisms with sources $\sigma_{j} \in \Delta(Y), j=1,2$.

- $\Gamma \in Z(\rho)$ and $\bar{\Gamma} \in Z(\bar{\rho})$ are conjugate to each other via the correspondence between $Z(\rho)$ and $Z(\bar{\rho})$ induced by $(r, \bar{r})$ (cf. Lemma 3.20$)$.

Notice that most of the subsequent computations (especially the proof of Lemma C.1) appear complicated only because we use the traditional 1-dimensional notation. They would be much more transparent in a 2-dimensional (graphical) notation. We also remind the reader of the convention regarding the monoidal product explained in on page 73 . (The convention is such that e.g. $r \otimes s t \circ u \equiv(r \otimes(s \circ t)) \circ u$. The compositions with explicitly written composition sign are to be evaluated last and thus indicate the coarsest substructure of a formula.)

For the sake of completeness, we begin with a general lemma which connects the symmetry $\epsilon=\epsilon^{Y}$, the statistical phase $\kappa_{\bar{\rho}}$ of $\bar{\rho}$ and the solution $(r, \bar{r}) \in \mathcal{R}(\rho, \bar{\rho})$.

Lemma C.1 Let $(r, \bar{r}) \in \mathcal{R}(\rho, \bar{\rho})$ be a standard solution. Then

$$
r^{*} \otimes \mathbf{1}_{\rho} \circ \kappa_{\bar{\rho} \otimes \epsilon_{\rho}} \circ r \otimes \mathbf{1}_{\rho}=\mathbf{1}_{\rho}
$$


Appendix C. Proof of Formula (4.4)

Proof: The morphism $\bar{r}$ determines a standard left inverse $\bar{\varphi}$ for $\bar{\rho}$. One therefore has from the definition of $\kappa_{\bar{\rho}}$ the identity $\kappa_{\bar{\rho}}=d(\bar{\rho}) \bar{\varphi}_{\bar{\rho}, \bar{\rho}}\left(\epsilon_{\bar{\rho}}\right)=\bar{r}^{*} \otimes \mathbf{1}_{\bar{\rho}} \circ \mathbf{1}_{\rho} \otimes \epsilon_{\bar{\rho}} \circ \bar{r} \otimes \mathbf{1}_{\bar{\rho}}$. This implies

$$
\begin{aligned}
& r^{*} \otimes \mathbf{1}_{\rho} \circ \kappa_{\bar{\rho} \otimes \epsilon_{\rho}} \circ r \otimes \mathbf{1}_{\rho}=\bar{r}^{*} \otimes r^{*} \mathbf{1}_{\rho} \circ \mathbf{1}_{\rho} \otimes \epsilon_{\bar{\rho}} \otimes \epsilon_{\rho} \circ \bar{r} \otimes r \otimes \mathbf{1}_{\rho}
\end{aligned}
$$

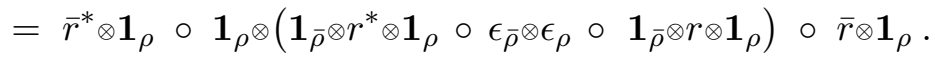

Now the term in the bracket can be rewritten as

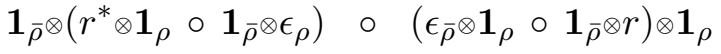

$$
\begin{aligned}
& \stackrel{(\bullet)}{=} \mathbf{1}_{\bar{\rho} \otimes}\left(\mathbf{1}_{\rho} \otimes r^{*} \circ \epsilon(\bar{\rho}, \rho) \otimes \mathbf{1}_{\rho}\right) \circ\left(\mathbf{1}_{\bar{\rho} \otimes \epsilon}(\rho, \bar{\rho}) \circ r \otimes \mathbf{1}_{\bar{\rho}}\right) \otimes \mathbf{1}_{\rho} \\
& =\mathbf{1}_{\bar{\rho} \rho \otimes r^{*}} \circ \mathbf{1}_{\bar{\rho} \otimes \epsilon}(\bar{\rho}, \rho) \epsilon(\rho, \bar{\rho}) \otimes \mathbf{1}_{\rho} \circ r \otimes \mathbf{1}_{\bar{\rho} \rho} \\
& =r \circ r^{*} \text {, }
\end{aligned}
$$

whence one obtains (postponing the justification of the equality marked with $(\bullet)$ )

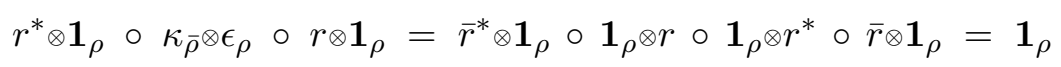

by the conjugate equations. To justify the transformation $(\bullet)$, one has to use the characteristic properties of the symmetry $\epsilon$ :

$$
r \otimes \mathbf{1}_{\bar{\rho}}=r \otimes \mathbf{1}_{\bar{\rho}} \circ \epsilon(\iota, \bar{\rho})=\epsilon(\bar{\rho}, \bar{\rho} \rho) \circ \mathbf{1}_{\bar{\rho} \otimes r}=\mathbf{1}_{\bar{\rho} \otimes \epsilon}(\bar{\rho}, \rho) \circ \epsilon(\bar{\rho}, \bar{\rho}) \otimes \mathbf{1}_{\rho} \circ \mathbf{1}_{\bar{\rho} \otimes r},
$$

which yields $\epsilon_{\bar{\rho} \otimes} \mathbf{1}_{\rho} \circ \mathbf{1}_{\bar{\rho} \otimes r}=\mathbf{1}_{\bar{\rho} \otimes \epsilon}(\rho, \bar{\rho}) \circ r \otimes \mathbf{1}_{\bar{\rho}}$. The other identity used in $(\bullet)$ is proved in a similar way.

Lemma C.1 can be viewed as a special case of the following one, for the proof of which it will be used.

Lemma C.2 Let $t_{j}: \sigma_{j} \rightarrow \rho(j=1,2)$. Then

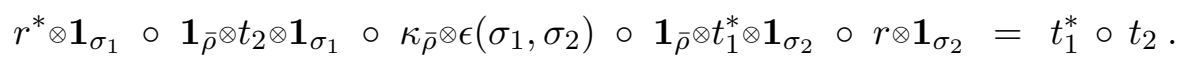

Proof: From the intertwining property of $\epsilon$ it follows that

$$
t_{2} \otimes \mathbf{1}_{\sigma_{1}} \circ \epsilon\left(\sigma_{1}, \sigma_{2}\right) \circ t_{1}^{*} \mathbf{1}_{\sigma_{2}}=\mathbf{1}_{\rho} \otimes t_{1}^{*} \circ \epsilon(\rho, \rho) \circ \mathbf{1}_{\rho} \otimes t_{2} .
$$

Taking the monoidal product of both sides with $\mathbf{1}_{\bar{\rho}} \circ \kappa_{\bar{\rho}} \circ \mathbf{1}_{\bar{\rho}}$ from the left and composing subsequently with $r^{*} \otimes \mathbf{1}_{\sigma_{1}}$ on the left and with $r \otimes \mathbf{1}_{\sigma_{2}}$ on the right, one obtains

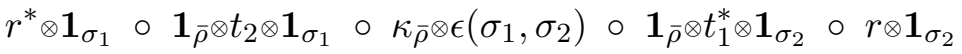

$$
\begin{aligned}
& =r^{*} \otimes \mathbf{1}_{\sigma_{1}} \circ \mathbf{1}_{\bar{\rho} \rho \otimes t_{1}^{*}} \circ \kappa_{\bar{\rho} \otimes \epsilon_{\rho}} \circ \mathbf{1}_{\bar{\rho} \rho \otimes t_{2}} \circ r \otimes \mathbf{1}_{\sigma_{2}} \\
& =t_{1}^{*} \circ r^{*} \otimes \mathbf{1}_{\rho} \circ \kappa_{\bar{\rho} \otimes \epsilon_{\rho}} \circ r \otimes \mathbf{1}_{\rho} \circ t_{2},
\end{aligned}
$$

which equals $t_{1}^{*} \circ t_{2}$ by Lemma C.1.

The only property of the objects $\sigma_{j}$ necessary for the above computation was that there is a common spacelike cone $Y$ in which $\sigma_{1}, \sigma_{2}$ and $\rho, \bar{\rho}$ are localised. This property still holds if each $\sigma_{j}$ is replaced with its image $\sigma_{j, x_{j}}=\beta_{x_{j}}\left(\sigma_{j}\right)$ under the translation $x_{j} \in \mathbb{R}^{1+s}$ (just replace $Y$ with $\left.\tilde{Y} \supset Y \cup\left(Y+x_{1}\right) \cup\left(Y+x_{2}\right)\right)$. The morphisms $\Gamma\left(x_{j}\right) \circ \beta_{x_{j}}\left(t_{j}\right)$ from $\sigma_{j, x_{j}}$ to $\rho$ can then be used instead of $t_{j}$. With these substitutions, Lemma C.2 yields: 


$$
\begin{aligned}
& \beta_{x_{1}}\left(t_{1}^{*}\right) \Gamma\left(x_{1}\right)^{*} \circ \Gamma\left(x_{2}\right) \beta_{x_{2}}\left(t_{2}\right)
\end{aligned}
$$

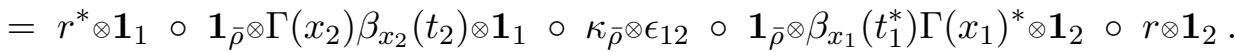

Here we are using the following abbreviations:

$$
\mathbf{1}_{j}:=\mathbf{1}_{\sigma_{j, x_{j}}}, \quad \mathbf{1}_{k l}:=\mathbf{1}_{k} \otimes \mathbf{1}_{l}, \quad \epsilon_{12}:=\epsilon\left(\sigma_{1, x_{1}}, \sigma_{2, x_{2}}\right) .
$$

The two leftmost and the two rightmost terms on the right-hand side of (C.2) can be simplified because of

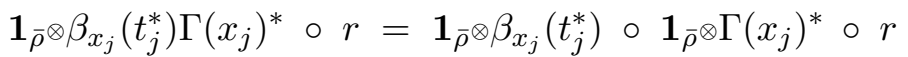

$$
\begin{aligned}
& =\mathbf{1}_{\bar{\rho}} \otimes \beta_{x_{j}}\left(t_{j}^{*}\right) \circ \bar{\Gamma}\left(x_{j}\right) \otimes \mathbf{1}_{\rho_{x_{j}}} \circ \beta_{x_{j}}(r)
\end{aligned}
$$

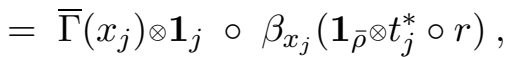

where the second equality uses (A.5) (for $r_{\tau} \equiv r, r_{\sigma} \equiv \beta_{x_{j}}(r), t \equiv \Gamma\left(x_{j}\right), t^{\dagger} \equiv \bar{\Gamma}\left(x_{j}\right)$ - see p. 84). One thus obtains from ( $\overline{\text { C.2 }}$ ) the following identity (valid for arbitrary $x_{j} \in \mathbb{R}^{1+s}$ ):

$$
\begin{aligned}
& \beta_{x_{1}}\left(t_{1}^{*}\right) \Gamma\left(x_{1}\right)^{*} \circ \Gamma\left(x_{2}\right) \beta_{x_{2}}\left(t_{2}\right)
\end{aligned}
$$

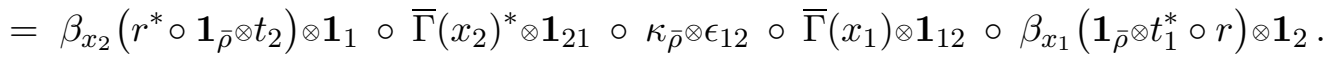

(Notice that the three terms in the middle could be written as $\bar{\Gamma}\left(x_{2}\right)^{*} \kappa_{\bar{\rho}} \bar{\Gamma}\left(x_{1}\right) \otimes \epsilon_{12}$, but that would not fit the present purposes.)

Next, we project the identity (C.4) to $\mathcal{B}\left(\mathcal{H}_{I}\right)$ via the map $t \longmapsto t$. Remembering that the morphism $\beta_{x}(t)$ is mapped to $\alpha_{x}(t)=\operatorname{Ad} U_{I}(x)(t)$ and using the notations

$$
\begin{gathered}
N\left(x_{1}, x_{2}\right):=\kappa_{\bar{\rho}} \bar{\rho}^{\tilde{Y}}\left(\epsilon\left(\sigma_{1, x_{1}}, \sigma_{2, x_{2}}\right)\right), \\
V(x):=\Gamma(x) U_{I}(x) \quad \text { and } \quad \bar{V}(x):=\bar{\Gamma}(x) U_{I}(x),
\end{gathered}
$$

we obtain (still for arbitrary $x_{j} \in \mathbb{R}^{1+s}$ )

$U_{I}\left(x_{1}\right) t_{1}^{*} V\left(x_{1}\right)^{*} V\left(x_{2}\right) t_{2} U_{I}\left(x_{2}\right)^{*}=U_{I}\left(x_{2}\right) r^{*} \bar{\rho}^{Y}\left(t_{2}\right) \bar{V}\left(x_{2}\right)^{*} N\left(x_{1}, x_{2}\right) \bar{V}\left(x_{1}\right) \bar{\rho}^{Y}\left(t_{1}^{*}\right) r U_{I}\left(x_{1}\right)^{*}$.

We can now return to the situation of Section 4.4. There one has $\sigma_{j} \in \Delta\left(X_{j}\right)$ for spacelike cones $X_{j} \subset Y$, and there is a wedge region $W$ such that $X_{1} \times\left(X_{2}+\bar{W}\right)$. Thus if $x_{2}-x_{1} \in \bar{W}$, then $\left(X_{1}+x_{1}\right) \times\left(X_{2}+x_{2}\right)$, implying that $\sigma_{1, x_{1}} \perp \sigma_{2, x_{2}}$ (in the notation of Section 3.4) and in particular $\epsilon\left(\sigma_{1, x_{1}}, \sigma_{2, x_{2}}\right)=\mathbf{1}_{12}=\mathbf{1}_{21}$. For these values of $x_{1}, x_{2}$, the operator $N\left(x_{1}, x_{2}\right)$ is thus given by $\kappa_{\bar{\rho}}$. The latter has the form $\kappa \mathbf{1}_{\mathcal{H}_{I}}$ with $\kappa \in\{ \pm 1\}$ if $\rho$ and $\bar{\rho}$ are irreducible (or just purely bosonic/fermionic, for that matter). Hence $N\left(x_{1}, x_{2}\right)=\kappa \mathbf{1}_{\mathcal{H}_{I}}$, and one therefore obtains formula (C.1) by setting $x_{1}=0$ and $x_{2}=x \in \bar{W}$.

Remarks: 1. The argument shows that formula (C.1) is independent of $\Gamma$ being the minimal element of $Z(\rho)$. Moreover, $\Gamma$ need not even be natural for (C.1) to be valid.

2. We could of course have worked with $x_{1}=0$ the whole time, but that would have hidden the symmetry present in the above formulae. 



\section{Appendix D}

\section{On Distributions Which Vanish in a Wedge Region}

This appendix is concerned with the Jost-Lehmann-Dyson (or wave equation) method for combining position and momentum space properties of a given tempered distribution in order to enlarge the set of points where this distribution is known to vanish.

The wave equation method belongs to the realm of real analysis but is closely related to methods (in particular techniques of analytic completion) which pertain to the theory of functions of several complex variables. We will comment briefly on the relation to these complex methods at the very end of this appendix.

Here we derive in particular (see Prop. D.6) the result used in Section 4.4 which says that a distribution vanishing in a wedge region $W$ is identically zero if the support of its Fourier transform is contained in a set of the form $G=\left(a_{+}+\overline{\mathbf{V}}_{+}\right) \cup\left(a_{-}+\overline{\mathbf{V}}_{-}\right)$with vectors $a_{ \pm} \in \mathbb{R}^{1+s}$ such that

$$
a_{+}-a_{-} \in \mathbf{V}_{+}+T_{W},
$$

$T_{W}$ being the subgroup of translations which leave $W$ invariant.

The wave equation method is not directly applicable unless $a_{+}-a_{-} \in \overline{\mathbf{V}}_{+}$, but as explained in the heuristic argument in Section 4.4, our generalisation will be obtained by considering suitable lower-dimensional situations and applying the method to these.

For the sake of consistency with Section 4.4 and for definiteness, we think of a distribution $f \in \mathcal{S}^{\prime}\left(\mathbb{R}^{1+s}\right)$ as being defined in position (Minkowski) space and of $\hat{f} \in \mathcal{S}^{\prime}\left(\mathbb{R}^{1+s}\right.$ ) on momentum space. We call supp $\hat{f}$ the spectrum of $f$.

\section{D.1 The JLD Correspondence}

The starting point of the wave equation technique is a bijection between distributions $f \in \mathcal{S}^{\prime}\left(\mathbb{R}^{1+s}\right)$ satisfying supp $\hat{f} \subset \overline{\mathbf{V}}=\overline{\mathbf{V}}_{+} \cup \overline{\mathbf{V}}_{-}$and solutions $F \in \mathcal{S}^{\prime}\left(\mathbb{R}^{1+(s+1)}\right)$ of the wave equation with an additional symmetry. This correspondence relies on the fact that the Minkowski spacetime $\mathbb{R}^{1+s}$ can be thought of as the $x_{s+1}=0$ subspace of the Minkowski spacetime $\mathbb{R}^{1+(s+1)}$; the latter is given the metric $x y=x_{0} y_{0}-x_{1} y_{1}-\cdots-x_{s} y_{s}-x_{s+1} y_{s+1}$, i.e., the additional direction is spacelike.

Thus via the embedding $\mathbb{R}^{1+s} \cong \mathbb{R}^{1+s} \times\{0\} \subset \mathbb{R}^{1+(s+1)}$, a point $x \in \mathbb{R}^{1+s}$ is identified with the point $(x, 0) \in \mathbb{R}^{1+(s+1)}$. Usually, no notational distinction will be necessary 
Appendix D. On Distributions Which Vanish in a Wedge Region

between points or subsets in $\mathbb{R}^{1+s}$ and their images under that embedding. As an example, we can write

$$
\breve{\mathbf{V}}_{ \pm} \cap\left(\mathbb{R}^{1+s} \times\{0\}\right)=\mathbf{V}_{ \pm}
$$

if $\breve{\mathbf{V}}_{ \pm}:=\left\{x \in \mathbb{R}^{1+(s+1)} \mid x^{2}>0, \pm x_{0}>0\right\}$ denotes the forward/backward light cone in $\mathbb{R}^{1+(s+1)}$.

Now let $\mathcal{O} \subset \mathbb{R}^{1+s}$ be an open double cone, i.e. $\mathcal{O}=\mathcal{O}_{a, b}:=\left(a+\mathbf{V}_{-}\right) \cap\left(b+\mathbf{V}_{+}\right)$, with $a, b \in \mathbb{R}^{1+s}, a-b \in \mathbf{V}_{+}$. Then the points $a, b \in \mathbb{R}^{1+(s+1)}$ define a double cone $\breve{\mathcal{O}} \subset \mathbb{R}^{1+(s+1)}$, namely $\breve{\mathcal{O}}=\breve{\mathcal{O}}_{a, b}=\left(a+\breve{\mathbf{V}}_{-}\right) \cap\left(b+\breve{\mathbf{V}}_{+}\right)$, and one has $\breve{\mathcal{O}} \cap\left(\mathbb{R}^{1+s} \times\{0\}\right)=\mathcal{O}$.

It is convenient to extend the mapping $\mathcal{O} \longmapsto \breve{\mathcal{O}}$ to arbitrary open subsets $G \subset \mathbb{R}^{1+s}$ :

$$
G \longmapsto \breve{G}:=\bigcup\{\breve{\mathcal{O}} \mid \mathcal{O} \text { is a double cone in } G\} \text {. }
$$

Then one still has $\breve{G} \cap\left(\mathbb{R}^{1+s} \times\{0\}\right)=G$.

Lemma D.1 The mapping $G \longmapsto \breve{G}$ has the following properties:

i. $G_{1} \subset G_{2}$ implies $\breve{G}_{1} \subset \breve{G}_{2}$.

ii. For arbitrary unions, one has $\left(\bigcup_{j} G_{j}\right)^{\iota} \supset \bigcup_{j} \breve{G}_{j}$.

iii. For finite intersections, one has $\left(\bigcap_{j} G_{j}\right)^{\iota} \subset \bigcap_{j} \breve{G}_{j}$.

iv. $\breve{G}=\bigcup\{\breve{\mathcal{O}} \mid \overline{\mathcal{O}} \subset G, \mathcal{O}$ a double cone $\}$.

v. If $G_{1} \subset G_{2} \subset G_{3} \subset \ldots$, then ii becomes $\left(\bigcup_{j} G_{j}\right)^{\cup}=\bigcup_{j} \breve{G}_{j}$.

vi. $(G+a)=\breve{G}+a$ for any translation $a \in \mathbb{R}^{1+s}$.

Proof: Property i is elementary and entails immediately ii and iii. Properties iv and vi can be reduced to the corresponding ones for double cones. Finally, the " $\subset$ " part of v not covered by ii follows as an application of iv to $G:=\bigcup_{j} G_{j}$, since any double cone $\mathcal{O}$ which fulfils $\overline{\mathcal{O}} \subset G$ is already contained in all but finitely many of the $G_{j}$ by a compactness argument.

Remark: We note that the inclusions in ii and iii are proper, in general. As an example for ii, let $\mathcal{O}$ be the double cone with vertices $( \pm R, \overrightarrow{0}), R>0$ and $A$ some (finite or infinite) set of translations in $\mathbb{R}^{1+s}$. Setting $G_{a}:=\mathcal{O}+a$, one has

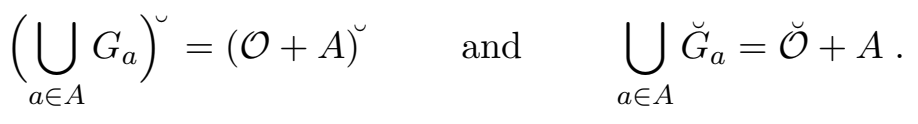

Now any $x \in \breve{\mathcal{O}}+A$ satisfies $\left|x_{s+1}\right|<R$, but if $\mathcal{O}+A$ contains a double cone $\tilde{\mathcal{O}}$ larger

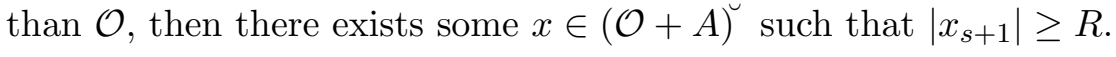

Property $\mathrm{v}$ of Lemma D.1 can be used to deduce that the map $(\breve{\cdot})$ maps the forward/backward light cones $\mathbf{V}_{ \pm}$of $\mathbb{R}^{1+s}$ to those of $\mathbb{R}^{1+(s+1)}$,

$$
\left(\mathbf{V}_{ \pm}\right)^{\breve{ }}=\breve{\mathbf{V}}_{ \pm}, \quad \breve{\mathbf{V}}=\breve{\mathbf{V}}_{+} \cup \breve{\mathbf{V}}_{-}
$$

which makes the notation coherent. Moreover, the notation can also consistently be extended to wedge regions defined (for given vectors $k_{ \pm} \in \partial \mathbf{V}_{ \pm}, k_{+} k_{-}<0$ ) by

$$
W_{k_{+}, k_{-}}:=\left\{x \in \mathbb{R}^{1+s} \mid k_{+} x<0, k_{-} x<0\right\}:
$$


if $\breve{W}_{k_{+}, k_{-}}$denotes the analogously defined wedge region in $\mathbb{R}^{1+(s+1)}$, then one has

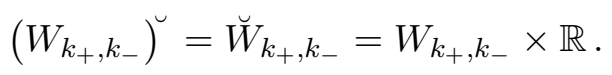

(To see this, notice that $W_{k_{+}, k_{-}}=\bigcup_{n \in \mathbb{N}} n \mathcal{O}_{k_{+}, k_{-}}$and that $0 \in \partial \mathcal{O}_{k_{+}, k_{-}}$. Therefore the first equality follows from Part $\mathrm{v}$ of Lemma D.1. The second equality is verified directly.)

As a last geometrical preparation regarding $\mathbb{R}^{1+(s+1)}$, it is necessary to introduce the reflection $\mathbb{R}^{1+(s+1)} \longrightarrow \mathbb{R}^{1+(s+1)}:\left(x_{0}, \ldots, x_{s}, x_{s+1}\right) \longmapsto\left(x_{0}, \ldots, x_{s},-x_{s+1}\right)$. Functions, distributions and subsets invariant under this reflection will be called symmetric. Occasionally, the term "symmetric" will also be applied to notions pertaining to the real line $\mathbb{R}$ if the latter is being considered as the subspace $\{0\} \times \mathbb{R} \subset \mathbb{R}^{1+s} \times \mathbb{R} \cong \mathbb{R}^{1+(s+1)}$.

With these notions, the aforementioned correspondence can now be formulated in the following proposition. We will refer to it as the JLD correspondence, the acronym standing for Jost-Lehmann-Dyson, since this correspondence is at the root of many mathematical facts associated with this combination of names.

Proposition D.2 A bijection between the spaces

$$
\left\{F \in \mathcal{S}^{\prime}\left(\mathbb{R}^{1+(s+1)}\right) \mid \square F=0, F \text { is symmetric }\right\} \quad \text { and } \quad\left\{f \in \mathcal{S}^{\prime}\left(\mathbb{R}^{1+s}\right) \mid \operatorname{supp} \hat{f} \subset \overline{\mathbf{V}}\right\}
$$

is given by the maps

$$
\begin{aligned}
& F \longmapsto f: f(\cdot)=F(\cdot, 0) \\
& f \longmapsto F: F(\cdot, \sigma)=\left(p \longmapsto \check{f}(p) \cos \left(\sigma \sqrt{p^{2}}\right)\right)
\end{aligned}
$$

Moreover, if $G \subset \mathbb{R}^{1+s}$ is open and if $f$ and $F$ correspond to each other, then one has

$$
\left.f\right|_{G}=0 \quad \text { iff }\left.\quad F\right|_{\breve{G}}=0
$$

and

$$
\operatorname{supp} \hat{F}=(\operatorname{supp} \hat{f} \times \mathbb{R}) \cap \partial \breve{\mathbf{V}}
$$

Proof: We refrain from repeating the proof of the first part of this theorem here since it can be found in the literature, e.g. Thm. III.4.2 in [15] (also see Chapter 4 in [41]). We recall however that $\square F=0$ implies that $\sigma \longmapsto F(\cdot, \sigma)$ is a smooth function from the real line $\mathbb{R}$ to the space $\mathcal{S}^{\prime}\left(\mathbb{R}^{1+s}\right)$ of distributions; in particular the expression $F(\cdot, 0)$ is well-defined.

The second part, too, is well known, but in view of its importance for the following, we will sketch a proof. Thus let $G \subset \mathbb{R}^{1+s}$ be open and assume $\left.F\right|_{\breve{G}}=0$. Then since $f=$ $\left.F\right|_{\mathbb{R}^{1+s} \times\{0\}}$ and $\breve{G} \cap\left(\mathbb{R}^{1+s} \times\{0\}\right)=G$, it follows that $\left.f\right|_{G}=0$. Conversely, assume $\left.f\right|_{G}=0$. By the definition of $\breve{G}$, it has to be shown that $\left.F\right|_{\breve{\mathcal{O}}}=0$ for any double cone $\mathcal{O} \subset G$. Now if $\mathcal{O}$ is such a double cone, then it follows from $\left.f\right|_{\mathcal{O}}=0$ that all partial derivatives $D^{\alpha} f$ $\left(\alpha=\left(\alpha_{0}, \ldots, \alpha_{s}\right)\right.$ a multi-index) of $f$ vanish throughout $\mathcal{O}$. Therefore, since $F$ satisfies the wave equation, all partial derivatives of the form $D^{\beta} F$ vanish throughout $\mathcal{O} \times\{0\}$ if the last component $\beta_{s+1}$ of the multi-index $\beta=\left(\beta_{0}, \ldots \beta_{s}, \beta_{s+1}\right)$ is even, and the same is trivially true if $\beta_{s+1}$ is odd since $F$ is symmetric. By Asgeirsson's lemma (Prop. 4.7 in [41]), these properties imply that $F$ vanishes in $\breve{\mathcal{O}}=(\mathcal{O} \times\{0\})^{\prime \prime}$.

Finally, the assertion about the supports in momentum space can be seen as follows. 
Appendix D. On Distributions Which Vanish in a Wedge Region

First notice that for test functions $\varphi_{1} \in \mathcal{S}\left(\mathbb{R}^{1+s}\right)$ and $\varphi_{2} \in \mathcal{S}(\mathbb{R}), \varphi_{2}$ symmetric, one has $\hat{F}\left(\varphi_{1} \otimes \varphi_{2}\right)=\int d p \hat{f}(p) \varphi_{1}(p) \varphi_{2}\left(\sqrt{p^{2}}\right)$. Now let $A_{1} \subset \mathbb{R}^{1+s}$ and $A_{2} \subset \mathbb{R}$ be open sets, $A_{2}$ symmetric. Then one has the following equivalent statements:

$$
\begin{aligned}
& \left(A_{1} \times A_{2}\right) \cap \operatorname{supp} \hat{F}=\emptyset \\
\Longleftrightarrow & \hat{F}\left(\varphi_{1} \otimes \varphi_{2}\right)=0 \text { for any } \varphi_{j} \text { as above with } \operatorname{supp} \varphi_{j} \subset A_{j}, j=1,2 \\
\Longleftrightarrow & \operatorname{supp} \hat{f} \cap A_{1} \cap\left\{p \in \overline{\mathbf{V}} \mid \sqrt{p^{2}} \in A_{2}\right\}=\emptyset \\
\Longleftrightarrow & \left(A_{1} \times A_{2}\right) \cap(\operatorname{supp} \hat{f} \times \mathbb{R}) \cap \partial \breve{\mathbf{V}}=\emptyset .
\end{aligned}
$$

Since $A_{1}$ and $A_{2}$ are arbitrary, this implies $\operatorname{supp} \hat{F}=(\operatorname{supp} \hat{f} \times \mathbb{R}) \cap \partial \breve{\mathbf{V}}$.

Remark and Notations: If $\pi: \partial \breve{\mathbf{V}} \rightarrow \overline{\mathbf{V}}$ denotes the canonical projection, then the relation between the spectra of $f$ and $F$ can be expressed as $\operatorname{supp} \hat{F}=\pi^{-1}(\operatorname{supp} \hat{f})$. Also notice that symmetric subsets of $\partial \breve{\mathbf{V}}$ are uniquely determined by their images under $\pi$. A particularly important class of symmetric subsets of $\partial \breve{\mathbf{V}}$ will be those of the form $\partial \breve{\mathbf{V}} \cap\left(\mathbb{R}^{1+s} \times I\right)$, where $I \subset \mathbb{R}$ is a symmetric open set. Their images under $\pi$ are the sets $\tilde{I}:=\left\{p \in \overline{\mathbf{V}} \mid \sqrt{p^{2}} \in I\right\}$, i.e., unions of hyperboloids with mass parameter in $I$. We will denote with $\tilde{I}_{ \pm}:=\tilde{I} \cap \overline{\mathbf{V}}_{ \pm}$the upper/lower part of $\tilde{I}$.

With the above notations, we obtain from the JLD correspondence a proposition about the spectral properties of distributions $f \in \mathcal{S}^{\prime}\left(\mathbb{R}^{1+s}\right)$ vanishing in a wedge region. It is in the same spirit as Thm. 6.3 of $[8]$.

Proposition D.3 Let $W \subset \mathbb{R}^{1+s}$ be a wedge region and let the distribution $f \in \mathcal{S}^{\prime}\left(\mathbb{R}^{1+s}\right)$ satisfy

$$
\operatorname{supp} \hat{f} \subset \overline{\mathbf{V}} \quad \text { and }\left.\quad f\right|_{W}=0 .
$$

Then one has for any open symmetric set $I \subset \mathbb{R}$ the equivalence

$$
\operatorname{supp} \hat{f} \cap \tilde{I}_{+}=\emptyset \quad \Longleftrightarrow \quad \operatorname{supp} \hat{f} \cap \tilde{I}_{-}=\emptyset .
$$

Proof: Due to the symmetry of the problem, it is sufficient to show one implication, say " $\Rightarrow$ ". Thus, assuming that $\operatorname{supp} \hat{f} \cap \tilde{I}_{+}=\emptyset, f$ corresponds via Prop. D.2 to a distribution $F \in \mathcal{S}^{\prime}\left(\mathbb{R}^{1+(s+1)}\right)$ with the properties

$$
\left.F\right|_{\breve{W}}=0 \quad \text { and } \operatorname{supp} \hat{F} \subset\left(\left(\mathbb{R}^{1+s} \backslash \tilde{I}_{+}\right) \times \mathbb{R}\right) \cap \partial \breve{\mathbf{V}} \subset\left(\left(\mathbb{R}^{1+s} \times(\mathbb{R} \backslash I)\right) \cap \partial \breve{\mathbf{V}}_{+}\right) \cup \partial \breve{\mathbf{V}}_{-} .
$$

Now let $u \in \mathcal{S}(\mathbb{R})$ be a symmetric (i.e., even) test function with supp $\hat{u} \subset I$ and consider the distribution $F_{u} \in \mathcal{S}^{\prime}\left(\mathbb{R}^{1+(s+1)}\right)$ defined by $\hat{F}_{u}=\hat{F} \cdot\left(\mathbf{1}_{\mathbb{R}^{1+s}} \otimes \hat{u}\right)$. Then the two abovementioned properties of $F$ imply for $F_{u}$ :

$$
\left.F_{u}\right|_{\breve{W}}=0 \quad \text { and } \quad \operatorname{supp} \hat{F}_{u} \subset \overline{\mathbf{V}_{-}} .
$$

(The assertion about momentum space follows from $\operatorname{supp} \hat{F}_{u} \subset \operatorname{supp} \hat{F} \cap\left(\mathbb{R}^{1+s} \times I\right) \subset \partial \breve{\mathbf{V}}_{-}$, whereas the one about position space is due to the fact that $F_{u}$ is the convolution of $F$ with the distribution $(x, \sigma) \longmapsto \delta(x) u(\sigma)$ and to the fact that $\breve{W}=W \times \mathbb{R}$ is invariant under translations in the $\sigma$-direction.) Because the support of $\hat{F}_{u}$ is contained in the cone $\overline{\mathbf{V}}_{-}$, the distribution $F_{u}$ is the boundary value of a function analytic in the tube $\mathbb{R}^{1+(s+1)}+i \breve{\mathbf{V}}_{-}$. Therefore it follows from $\left.F_{u}\right|_{\breve{W}}=0$ that $F_{u}$ vanishes identically. Varying $u$ now leads to $\operatorname{supp} \hat{F} \cap\left(\mathbb{R}^{1+s} \times I\right)=\emptyset$, which is equivalent to $\operatorname{supp} \hat{f} \cap \tilde{I}=\emptyset$ via Prop. D.2. 


\section{D.2 Criteria Making $f$ Vanish}

From now on, we work in the physical Minkowski space $\mathbb{R}^{1+s}$ only. We consider distributions $f \in \mathcal{S}^{\prime}\left(\mathbb{R}^{1+s}\right)$ which vanish in a wedge region $W \subset \mathbb{R}^{1+s}$ and look for conditions on supp $\hat{f}$ which entail that $f$ vanishes. A rather trivial one is expressed in Lemma D.4. It follows from Prop. D.3 and is insensitive to the choice of $W$. It will be used below to derive less trivial ones which of course do depend on $W$.

Lemma D.4 Let $f \in \mathcal{S}^{\prime}\left(\mathbb{R}^{1+s}\right)$ be a distribution which vanishes in some wedge region. If there exist $a_{ \pm} \in \mathbb{R}^{1+s}$, $a_{+}-a_{-} \in \mathbf{V}_{+}$, such that $\operatorname{supp} \hat{f} \subset\left(a_{+}+\mathbf{V}_{+}\right) \cup\left(a_{-}+\mathbf{V}_{-}\right)$, then one has $f=0$.

Proof: Denote with $f_{ \pm}$the distributions obtained from $f$ by translations by $-a_{ \pm}$in momentum space, i.e., $\hat{f}_{ \pm}(p)=\hat{f}\left(p+a_{ \pm}\right)$. Setting $q:=a_{+}-a_{-}$, one has

$$
\operatorname{supp} \hat{f}_{ \pm} \subset \mathbf{V}_{ \pm} \cup\left(\mathbf{V}_{\mp} \mp q\right), \quad \operatorname{supp} \hat{f}_{\mp}=\operatorname{supp} \hat{f}_{ \pm} \pm q .
$$

We now show by induction on $k$ that $\operatorname{supp} \hat{f}_{ \pm} \cap k \tilde{I}=\emptyset$ for all $k \in \mathbb{N}$. Here $I$ is the symmetric interval $I:=]-\sqrt{q^{2}},+\sqrt{q^{2}}[$ :

For $k=1$, one has $\operatorname{supp} \hat{f}_{ \pm} \cap \tilde{I}_{\mp} \subset\left(\mathbf{V}_{\mp} \mp q\right) \cap \tilde{I}_{\mp}=\mp\left(\left(\mathbf{V}_{+}+q\right) \cap \tilde{I}_{+}\right)=\emptyset$. Since $f_{ \pm}$vanish on the same wedge region as $f$, Prop. D.3 implies $\operatorname{supp} \hat{f}_{ \pm} \cap \tilde{I}=\emptyset$.

Now let $k \in \mathbb{N}$ be arbitrary. Then

$$
\begin{aligned}
& \operatorname{supp} \hat{f}_{ \pm} \cap k \tilde{I}_{ \pm}=\emptyset \\
\Longrightarrow & \operatorname{supp} \hat{f}_{ \pm} \subset\left(\mathbf{V}_{ \pm} \backslash k \tilde{I}_{ \pm}\right) \cup\left(\mathbf{V}_{\mp} \mp q\right) \\
\Longrightarrow & \operatorname{supp} \hat{f}_{\mp} \subset\left(\left(\mathbf{V}_{ \pm} \backslash k \tilde{I}_{ \pm}\right) \pm q\right) \cup \mathbf{V}_{\mp} \\
\Longrightarrow & \operatorname{supp} \hat{f}_{\mp} \cap \mathbf{V}_{ \pm} \subset\left(\mathbf{V}_{ \pm} \backslash k \tilde{I}_{ \pm}\right) \pm q \subset \mathbf{V}_{ \pm} \backslash(k+1) \tilde{I}_{ \pm} \\
\Longrightarrow & \operatorname{supp} \hat{f}_{\mp} \cap(k+1) \tilde{I}_{ \pm}=\emptyset,
\end{aligned}
$$

where the properties (D.1) of $\hat{f}$ have been used in the first two implications and the geometry of $\tilde{I}_{ \pm}$in the fourth line. Prop. D.3 now implies supp $\hat{f}_{\mp} \cap(k+1) \tilde{I}=\emptyset$, which completes the induction. As $\bigcup_{k \in \mathbb{N}} k \tilde{I}=\overline{\mathbf{V}}$, this means $\operatorname{supp} \hat{f}_{ \pm}=\emptyset$, hence $f=0$.

In order to formulate the nontrivial generalisation of this lemma, we need some terminology and notations in the context of wedge regions.

Notation: Any (open) wedge region has the form $W=w+W_{k_{+}, k_{-}}$with $w \in \mathbb{R}^{1+s}$ and with $k_{ \pm} \in \partial \mathbf{V}_{ \pm}$satisfying $k_{+} k_{-}<0$. It defines a two-dimensional timelike plane $\mathcal{M}_{\|}(W):=\operatorname{span}\left\{k_{+}, k_{-}\right\}$through the origin. Its orthogonal complement', the spacelike plane $\mathcal{M}_{\perp}(W):=\left\{y \in \mathbb{R}^{1+s} \mid y k_{ \pm}=0\right\}$, coincides exactly with the group of translations which leave the region $W$ invariant. (The set $w+\mathcal{M}_{\perp}(W)=\bar{W} \cap \overline{W^{\prime}}$ is just the edge of $W$; it can be characterised equivalently as the largest affine subspace contained in $\partial W$.) Notice that $W$ induces an isomorphism $\mathbb{R}^{1+s} \cong \mathcal{M}_{\|}(W) \oplus \mathcal{M}_{\perp}(W)$; we will denote the image of $y \in \mathbb{R}^{1+s}$ with $\left(y_{\|}, y_{\perp}\right)$.

The following proposition embodies the idea that the set of known zeros of $\hat{f}$ can be enlarged by scanning momentum space with (fattened) two-dimensional planes parallel to $\mathcal{M}_{\|}(W)$

\footnotetext{
${ }^{1}$ As a mnemonic for not confusing $\mathcal{M}_{\|}$and $\mathcal{M}_{\perp}$, think of the two bars $\|$ as standing for $\mathcal{M}_{\|}$being two-dimensional.
} 
Appendix D. On Distributions Which Vanish in a Wedge Region

Proposition D.5 Let $f \in \mathcal{S}^{\prime}\left(\mathbb{R}^{1+s}\right)$ be a distribution which vanishes in the wedge region $W$, and let $\mathcal{N} \subset \mathbb{R}^{1+s}$ be an open set invariant under the translations in $\mathcal{M}_{\|}(W)$. If there exist $a_{ \pm} \in \mathbb{R}^{1+s}, a_{+}-a_{-} \in \mathbf{V}_{+}$, such that $\operatorname{supp} \hat{f} \cap \mathcal{N} \subset\left(a_{+}+\mathbf{V}_{+}\right) \cup\left(a_{-}+\mathbf{V}_{-}\right)$, then $\left.\hat{f}\right|_{\mathcal{N}}=0$.

Proof: The set $\mathcal{N}$ has the form $\mathcal{N}=\mathcal{M}_{\|}(W) \times \mathcal{N}_{\perp}$, where $\mathcal{N}_{\perp}$ is open in $\mathcal{M}_{\perp}(W)$. Let $u_{\perp}: \mathcal{M}_{\perp}(W) \rightarrow \mathbb{C}$ be a test function with compact support in $\mathcal{N}_{\perp}$, and let the smooth function $u: \mathbb{R}^{1+s} \rightarrow \mathbb{C}$ be defined by $u(p):=u_{\perp}\left(p_{\perp}\right)$. Then the distribution $\hat{f} u \in \mathcal{S}^{\prime}\left(\mathbb{R}^{1+s}\right)$ satisfies

$$
\operatorname{supp}(\hat{f} u) \subset \operatorname{supp} \hat{f} \cap \mathcal{N} \subset\left(a_{+}+\mathbf{V}_{+}\right) \cup\left(a_{-}+\mathbf{V}_{-}\right) .
$$

The invariance of $u$ under $\mathcal{M}_{\|}(W)$ now implies that $\hat{f} u$ is the Fourier transform of the convolution product $f *\left(\delta_{\|} \otimes \check{u}_{\perp}\right)$ (where $\left(\delta_{\|} \otimes \check{u}_{\perp}\right)(x)=\delta^{(2)}\left(x_{\|}\right) \check{u}_{\perp}\left(x_{\perp}\right)$ ), which is a distribution with support in

$$
\operatorname{supp} f+\operatorname{supp}\left(\delta_{\|} \otimes \check{u}_{\perp}\right) \subset\left(\mathbb{R}^{1+s} \backslash W\right)+\mathcal{M}_{\perp}(W)=\mathbb{R}^{1+s} \backslash W .
$$

Thus $(\hat{f} u)^{\swarrow}$ also vanishes in $W$, so $\hat{f} u=0$ by Lemma D.4. Since $u$ was arbitrary (within the above specifications), this yields the assertion.

A criterion on supp $\hat{f}$ which makes $f$ vanish is now obvious: if (a set $G$ known to contain) $\operatorname{supp} \hat{f}$ can be covered with sets $\mathcal{N}$ such as in Prop. D.5, then that proposition can be invoked locally. We formalise as follows the relevant property of $G$ :

Definition: Let $\mathcal{M}_{\|}$be a two-dimensional timelike plane in $\mathbb{R}^{1+s}$. A set $G \subset \mathbb{R}^{1+s}$ is said to have property $\operatorname{lts}\left(\mathcal{M}_{\|}\right)$iff every $p \in G$ has an open neighbourhood $\mathcal{N}_{p}$ invariant under $\mathcal{M}_{\|}$such that

$$
G \cap \mathcal{N}_{p} \subset\left(a_{+}(p)+\mathbf{V}_{+}\right) \cup\left(a_{-}(p)+\mathbf{V}_{-}\right)
$$

with suitable $a_{ \pm}(p) \in \mathbb{R}^{1+s}, a_{+}(p)-a_{-}(p) \in \mathbf{V}_{+}$. (The letters lts stand for "locally timelike separated", since in restriction to each $\mathcal{N}_{p}$, the set $G$ is the union of two timelike separated subsets.)

In view of this definition and the preceding remarks, the proof of the following proposition is straightforward (and therefore omitted):

Proposition D.6 Let $f \in \mathcal{S}^{\prime}\left(\mathbb{R}^{1+s}\right)$ be a distribution which vanishes in the wedge region $W$. If $\operatorname{supp} \hat{f} \subset G$ where the set $G$ has property $\operatorname{lts}\left(\mathcal{M}_{\|}(W)\right)$, then $f$ vanishes identically.

In view of Prop. D.6 it is important to know examples of sets $G$ which have property $\operatorname{lts}\left(\mathcal{M}_{\|}\right)$for some given timelike 2-plane $\mathcal{M}_{\|}$. Trivially, $G=\left(a_{+}+\overline{\mathbf{V}}_{+}\right) \cup\left(a_{-}+\overline{\mathbf{V}}_{-}\right)$with $a_{+}-a_{-} \in \mathbf{V}_{+}$has this property, regardless of the choice of $\mathcal{M}_{\|}$. Moreover, the union of all translates of $G$ by vectors orthogonal to $\mathcal{M}_{\|}$still satisfies $\operatorname{lts}\left(\mathcal{M}_{\|}\right)$, and for this union only the component of $\left(a_{+}-a_{-}\right)$parallel to $\mathcal{M}_{\|}$is relevant. We state this as a geometrical lemma:

Lemma D.7 Let $\mathcal{M}_{\|}$be a timelike 2-plane in $\mathbb{R}^{1+s}$ and $\mathcal{M}_{\perp}$ its orthogonal complement. Let $a_{ \pm} \in \mathbb{R}^{1+s}$ fulfil $\left(a_{+}-a_{-}\right)_{\|} \in \mathbf{V}_{+}$. Then the set $G:=\left(\left(a_{+}+\overline{\mathbf{V}}_{+}\right) \cup\left(a_{-}+\overline{\mathbf{V}}_{-}\right)\right)+\mathcal{M}_{\perp}$ has property $\operatorname{lts}\left(\mathcal{M}_{\|}\right)$. 
Proof: After a translation by $-\frac{1}{2}\left(a_{+}+a_{-}\right)$we can assume that $a_{ \pm}= \pm c$, where $c_{\|} \in \mathbf{V}_{+}$. We then have to show that $\left(c+\overline{\mathbf{V}}_{+}+\mathcal{M}_{\perp}\right) \cup-\left(c+\overline{\mathbf{V}}_{+}+\mathcal{M}_{\perp}\right)$ has property $\operatorname{lts}\left(\mathcal{M}_{\|}\right)$. Let $q:=c_{\|}$and $K:=\left\{v \in \mathcal{M}_{\perp} \mid v^{2}>-\frac{q^{2}}{4}\right\}$. For any $p \in \mathbb{R}^{1+s}$, let $a_{ \pm}(p):=p_{\perp} \pm \frac{q}{2}$ and $\mathcal{N}_{p}:=p_{\perp}+K+\mathcal{M}_{\|}$. Then $a_{+}(p)-a_{-}(p)=q \in \mathbf{V}_{+}$, and since $K$ is an open neighbourhood of 0 in $\mathcal{M}_{\perp}, \mathcal{N}_{p}$ is an open, $\mathcal{M}_{\|}$-invariant neighbourhood of $p$. It remains to show that

$$
\pm\left(c+\overline{\mathbf{V}}_{+}+\mathcal{M}_{\perp}\right) \cap \mathcal{N}_{p} \subset a_{ \pm}(p)+\mathbf{V}_{ \pm} .
$$

Since $\pm c-a_{ \pm}(p)= \pm c_{\perp} \pm q-p_{\perp} \mp \frac{q}{2} \in \pm \frac{q}{2}+\mathcal{M}_{\perp}$ and $\mathcal{N}_{p}-a_{ \pm}(p)=K+\mathcal{M}_{\|}$, this is equivalent (for either sign) to

$$
\left(\frac{q}{2}+\overline{\mathbf{V}}_{+}+\mathcal{M}_{\perp}\right) \cap\left(K+\mathcal{M}_{\|}\right) \subset \mathbf{V}_{+} .
$$

This last relation is verified in a direct computation: Let $y \in\left(\frac{q}{2}+\overline{\mathbf{V}}_{+}+\mathcal{M}_{\perp}\right) \cap\left(K+\mathcal{M}_{\|}\right)$. Then $y \in \frac{q}{2}+\overline{\mathbf{V}}_{+}+\mathcal{M}_{\perp}$ implies $y_{\|} \in \frac{q}{2}+\overline{\mathbf{V}}_{+}$, hence $y_{\|}^{2} \geq \frac{q^{2}}{4}$. On the other hand, $y \in K+\mathcal{M}_{\|}$ implies $y_{\perp} \in K$, hence $y_{\perp}^{2}>-\frac{q^{2}}{4}$. This means that $y^{2}=y_{\|}^{2}+y_{\perp}^{2}>0$, i.e. $y \in \mathbf{V}_{+} \cup \mathbf{V}_{-}$. Together with $y_{\|} \in \overline{\mathbf{V}}_{+}$, this implies $y \in \mathbf{V}_{+}$.

Notice that the set $G$ considered in Lemma D.7 has an alternative geometric description in terms of wedges. Let $\pm W$ denote the two wedge regions whose edge is $\mathcal{M}_{\perp}$ and let $\mathcal{O}$ be the double cone with vertices $\left(a_{ \pm}\right)_{\|}$. Then

$$
\left(\left(a_{+}+\overline{\mathbf{V}}_{+}\right) \cup\left(a_{-}+\overline{\mathbf{V}}_{-}\right)\right)+\mathcal{M}_{\perp}=\mathbb{R}^{1+s} \backslash(\mathcal{O}+(W \cup-W))
$$

\section{D.3 Further Generalisations}

The methods of the previous section admit several quite obvious generalisations. Although these are not needed for obtaining results with relevance to physics in the main text, we want to put some of them on record here since they might turn out to be useful in other contexts.

To this end, it is convenient to slightly reformulate Prop. D.5 in a way which formalises more directly that it constitutes a method of improving the known localisation of supp $\hat{f}$. For a subset $G \subset \mathbb{R}$ and a timelike 2 -plane $\mathcal{M}_{\|}$let us define

$$
r_{\mathcal{M}_{\|}}(G):=G \backslash \bigcup \mathcal{N}
$$

where the union runs through all open sets $\mathcal{N} \subset \mathbb{R}^{1+s}$ invariant under $\mathcal{M}_{\|}$and such that $G \cap \mathcal{N} \subset\left(a_{+}+\mathbf{V}_{+}\right) \cup\left(a_{-}+\mathbf{V}_{-}\right)$for suitable $a_{ \pm} \in \mathbb{R}^{1+s}, a_{+}-a_{-} \in \mathbf{V}_{+}$. We will also write $r_{W}(G):=r_{\mathcal{M}_{\|}(W)}(G)$ if $W$ is a wedge region. With this notation, Prop. D.5 takes the following form:

Proposition D.8 Let $f \in \mathcal{S}^{\prime}\left(\mathbb{R}^{1+s}\right)$ be a distribution which vanishes in the wedge region $W$. If $\operatorname{supp} \hat{f} \subset G$, then $\operatorname{supp} \hat{f} \subset r_{W}(G)$.

\section{Remarks:}

1. $r_{W}(G)=\emptyset$ iff $G$ has property $\operatorname{lts}\left(\mathcal{M}_{\|}(W)\right)$; thus Prop. D.8 reproduces Prop. D.6.

2. $r_{W}(G)$ is closed if $G$ is closed; 
3. $r_{W}$ is contracting: $r_{W}(G) \subset G$;

4. $r_{W}$ is isotonous: $r_{W}\left(G_{1}\right) \subset r_{W}\left(G_{2}\right)$ if $G_{1} \subset G_{2}$;

5. $r_{W}$ is not idempotent: there are sets $G$ such that $r_{W}\left(r_{W}(G)\right) \subsetneq r_{W}(G)$, as will be seen in the next example.

Example: We will exhibit a set $G$ such that $r_{W}(G) \neq \emptyset$ but $r_{W}\left(r_{W}(G)\right)=\emptyset$. For definiteness consider in $\mathbb{R}^{1+s}=\mathbb{R}^{1+2}$ the wedge $W=\left\{x \in \mathbb{R}^{1+2}\left|x^{1}>\right| x^{0} \mid\right\}$, so $\mathcal{M}_{\|}(W)=$ $\left\{x \in \mathbb{R}^{1+2} \mid x^{2}=0\right\}$. Denote with $C:=\mathcal{M}_{\|}(W) \cap \mathbf{V}_{+}=\left\{x \in \mathbb{R}^{1+2}\left|x^{0}>\right| x^{1} \mid, x^{2}=0\right\}$ the forward light cone in $\mathcal{M}_{\|}(W)$. Now let $G:=G_{1} \cup G_{2} \cup G_{3}$, where each of the sets $G_{j}$ is of the form $G_{j}:=\left(\ell_{j}^{+}+C\right) \cup\left(\ell_{j}^{-}-C\right)$ with the (half-) lines $\ell_{j}^{ \pm}$given by

$$
\begin{aligned}
& \ell_{1}^{ \pm}=\{( \pm 1,+2, y) \mid y>0\}, \\
& \ell_{2}^{ \pm}=\{( \pm 1,-2, y) \mid y<0\}, \\
& \ell_{3}^{ \pm}=\{( \pm 3,0, y) \mid y \in \mathbb{R}\} .
\end{aligned}
$$

Since $G \cap\left\{x \in \mathbb{R}^{1+2} \mid \pm x^{2}>0\right\}=G_{1,2}$ and $G_{1,2}$ have property $\operatorname{lts}\left(\mathcal{M}_{\|}(W)\right)$, one readily sees that $r_{W}(G)=G_{3} \cap \mathcal{M}_{\|}(W)$ and $r_{W}\left(r_{W}(G)\right)=\emptyset$.

The above set $G$ is open, but there also exist closed sets with the property in question, e.g. the set $\left(\left(\overline{G_{1}} \cup \overline{G_{2}}\right) \cap B\right) \cup \overline{G_{3}}$, where $B:=\left\{x \in \mathbb{R}^{1+2}|| x^{2}|| x^{0} \mid \geq 1\right\}$.

The straightforward generalisation of Prop. D.8 now consists in applying it to situations where more information in position space is available in order to obtain more information in momentum space. More precisely, let us assume that $f \in \mathcal{S}^{\prime}\left(\mathbb{R}^{1+s}\right)$ vanishes in a set which contains the union of several wedges, i.e., $\left.f\right|_{\cup \mathcal{L}}=0$, where $\mathcal{L}$ is some collection of wedge regions. By applying Prop. $\mathbb{D} .8$ to each $W \in \mathcal{L}$, one obtains in this case from $\operatorname{supp} \hat{f} \subset G$ the stronger information $\operatorname{supp} \hat{f} \subset r_{\mathcal{L}}(G)$, where $r_{\mathcal{L}}(G):=\bigcap_{W \in \mathcal{L}} r_{W}(G)$. Since this argument can be iterated, it follows that $\operatorname{supp} \hat{f}$ is contained in each set in the following sequence:

$$
G \supset r_{\mathcal{L}}(G) \supset r_{\mathcal{L}}^{2}(G) \supset \ldots \supset r_{\mathcal{L}}^{\infty}(G):=\bigcap_{k \in \mathbb{N}} r_{\mathcal{L}}^{k}(G)
$$

Summarising, one has

Proposition D.9 Let $f \in \mathcal{S}^{\prime}\left(\mathbb{R}^{1+s}\right)$ be a distribution which vanishes in $\bigcup \mathcal{L}$, where $\mathcal{L}$ is some collection of wedge regions. If $\operatorname{supp} \hat{f} \subset G$, then $\operatorname{supp} \hat{f} \subset r_{\mathcal{L}}^{\infty}(G)$.

The properties of the map $r_{\mathcal{L}}^{\infty}$ follow from those of $r_{W}: r_{\mathcal{L}}^{\infty}(G)$ is closed if $G$ is closed. Like $r_{W}, r_{\mathcal{L}}^{\infty}$ is contracting and isotonous, and due to the infinite iteration involved, it is also idempotent.

Proposition D.9 seems to be the best result obtainable when only the information encoded in Lemma D.4 is being used. But as the latter is quite a weak consequence of Prop. D.3, the above results can be improved considerably by replacing $r_{\mathcal{M}_{\|}}$with some map $\tilde{r}_{\mathcal{M}_{\|}}$which still makes the analogue of Prop. D.8 true and which incorporates the full geometrical content of Prop. D.3. More specifically, this can be done as follows:

Definition: Let $W$ be a wedge region. A set $\mathcal{N} \subset \mathbb{R}^{1+s} \cong \mathcal{M}_{\|}(W) \times \mathcal{M}_{\perp}(W)$ of the form $\mathcal{N}=\left(\mathcal{M}_{\|}(W) \times \mathcal{N}_{\perp}\right) \cap(b+\tilde{I})$, where $\mathcal{N}_{\perp}$ is an open subset of $\mathcal{M}_{\perp}(W), b \in \mathbb{R}^{1+s}$ 
and where $\tilde{I}$ arises from a symmetric open set $I \subset \mathbb{R}$ as defined before Prop. D.3, will be called suitable ${ }^{2}$ for $(G, W)$, where $G$ is some subset of $\mathbb{R}^{1+s}$, if

- $G \cap\left(\mathcal{M}_{\|}(W) \times \mathcal{N}_{\perp}\right) \subset b+\overline{\mathbf{V}}$ and

- $G \cap\left(\mathcal{M}_{\|}(W) \times \mathcal{N}_{\perp}\right) \cap\left(b+\tilde{I}_{ \pm}\right)=\emptyset \quad$ for at least one of the two signs.

Moreover let $\tilde{r}_{W}(G):=G \backslash \bigcup \mathcal{N}$, where $\mathcal{N}$ runs through all sets suitable for $(G, W)$.

By mimicking the proof of Prop. D.4, one now obtains the following improvement of Proposition D.8:

Proposition D.10 Let $f \in \mathcal{S}^{\prime}\left(\mathbb{R}^{1+s}\right)$ be a distribution which vanishes in the wedge region $W$. If $\operatorname{supp} \hat{f} \subset G$, then $\operatorname{supp} \hat{f} \subset \tilde{r}_{W}(G)$.

Proof: We write $\mathcal{M}_{\|}:=\mathcal{M}_{\|}(W)$ and $\mathcal{M}_{\perp}:=\mathcal{M}_{\perp}(W)$. Let $\mathcal{N}=\left(\mathcal{M}_{\|} \times \mathcal{N}_{\perp}\right) \cap(b+\tilde{I})$ be suitable for $(G, W)$. Then we have to show that $\left.\hat{f}\right|_{\mathcal{N}}=0$. Choose a test function $u_{\perp}: \mathcal{M}_{\perp} \rightarrow \mathbb{C}$ with compact support in $\mathcal{N}_{\perp}$ and define the smooth function $u: \mathbb{R}^{1+s} \rightarrow \mathbb{C}$ by $u(p):=u_{\perp}\left(p_{\perp}\right)$. Then the distribution $\hat{f} u$ satisfies

$$
\operatorname{supp}(\hat{f} u) \subset G \cap\left(\mathcal{M}_{\|} \times \mathcal{N}_{\perp}\right) \subset b+\overline{\mathbf{V}},
$$

and since $\mathcal{N}$ is suitable for $(G, W)$ one has for at least one of the two signs

$$
\operatorname{supp}(\hat{f} u) \cap\left(b+\tilde{I}_{ \pm}\right) \subset G \cap\left(\mathcal{M}_{\|} \times \mathcal{N}_{\perp}\right) \cap\left(b+\tilde{I}_{ \pm}\right)=\emptyset .
$$

Now the same convolution argument as in the proof of Prop. D.5 shows that $(\hat{f} u)^{\swarrow}$ vanishes in $W$. Therefore Prop. D.3 can be applied (after a trivial translation by $-b$ in momentum space) and yields $\operatorname{supp}(\hat{f} u) \cap(b+\tilde{I})=\emptyset$. Since $u$ was arbitrary within the above specifications, one obtains supp $\hat{f} \cap\left(\mathcal{M}_{\|} \times \mathcal{N}_{\perp}\right) \cap(b+\tilde{I})=\emptyset$, i.e., $\operatorname{supp} \hat{f} \cap \mathcal{N}=\emptyset$, which was to be shown.

The algebraic and topological properties of $\tilde{r}_{W}$ are similar to those of $r_{W}$. With the obvious definitions of $\tilde{r}_{\mathcal{L}}$ and $\tilde{r}_{\mathcal{L}}^{\infty}$ one can therefore state the following result:

Proposition D.11 Let $f \in \mathcal{S}^{\prime}\left(\mathbb{R}^{1+s}\right)$ be a distribution which vanishes in $\bigcup \mathcal{L}$, where $\mathcal{L}$ is some collection of wedge regions in $\mathbb{R}^{1+s}$. If $\operatorname{supp} \hat{f} \subset G$, then $\operatorname{supp} \hat{f} \subset \tilde{r}_{\mathcal{L}}^{\infty}(G)$.

Let us end this appendix with a brief remark on the connection of the above results with the theory of functions of several complex variables. There the problem of combining position and momentum space information of a given distribution corresponds to a problem of analytic continuation. More precisely, the zeros of $\hat{f}$ appear as certain real boundary points ("coincidence points") of the holomorphy envelope $H(\mathcal{R})$ of a certain subset $\mathcal{R} \subset$ $\mathbb{C}^{(1+s)}$ (of complexified momentum space) which in turn is determined by the data $\mathbb{R}^{1+s} \backslash G$ and $\bigcup \mathcal{L}$ (in the above notations). The problem of analytic continuation then consists in finding (a suitably large subset of) $H(\mathcal{R})$, given $\mathcal{R}$. While this analytic technique is potentially very powerful, it turns out to be quite difficult in practice, especially if it leads (as in the present cases) to so-called oblique (and possibly flat) Edge of the Wedge situations (cf. [42]). We thus believe that the wave equation techniques presented here have the advantage of being geometrically simpler, although their results are weaker in general. There are in fact strong indications that our sets $\mathbb{R}^{1+s} \backslash \tilde{r}_{\mathcal{L}}^{\infty}(G)$ are always contained in the set of coincidence points of $H(\mathcal{R})$. We hope to clarify some of these issues in future work.

\footnotetext{
${ }^{2}$ Although bearing some superficial geometric resemblance, this notion should definitely not be confused with that of "admissible" hyperboloids which play an important role for the JLD theorem.
} 



\section{Bibliography}

[1] Muon $(g-2)$ collaboration. Precise measurement of the positive muon anomalous magnetic moment, hep-ex/0102017.

[2] R.F.Streater, A.S. Wightman. PCT, Spin and Statistics, and All That, Benjamin 1964.

[3] R. Haag. Local Quantum Physics, 2nd edition, Springer 1996.

[4] D. Buchholz. Algebraic Quantum Field Theory, monograph still to be written, meanwhile see [3, 43, 44, 45].

[5] K. Kraus, L. Polley, G. Reents. Models for Infrared Dynamics: I. Classical Currents, Ann. Inst. H. Poincaré A 26 (1977) 109-162.

[6] D. Buchholz. The Physical State Space of Quantum Electrodynamics, Commun. Math. Phys. 85 (1982) 49-71.

[7] S. Doplicher, R. Haag, J.E. Roberts. Local Observables and Particle Statistics I, Commun. Math. Phys. 23 (1971) 199-230.

[8] S. Doplicher, R. Haag, J.E. Roberts. Local Observables and Particle Statistics II, Commun. Math. Phys. 35 (1974) 49-85.

[9] D. Buchholz, K. Fredenhagen. Locality and the Structure of Particle States, Commun. Math. Phys. 84 (1982) 1-54.

[10] R. Haag, D. Kastler. An Algebraic Approach to Quantum Field Theory, J. Math. Phys. 5 (1964) 848-861.

[11] C. d'Antoni. Technical Properties of the Quasi-Local Algebra, in "The Algebraic Theory of Superselection Sectors. Introduction and Recent Results", 248-258; ed.: D. Kastler, World Scientific 1989.

[12] H.-J. Borchers. Translation Group and Spectrum Condition, Commun. Math. Phys. 96 (1984) 1-13.

[13] H.-J. Borchers, D. Buchholz. The Energy-Momentum Spectrum in Local Field Theories with Broken Lorentz-Symmetry, Commun. Math. Phys. 97 (1985) 169-185.

[14] H.-J. Borchers. Locality and covariance of the spectrum, Fizika 17 (1985) 289-304.

[15] H.-J. Borchers. Translation Group and Particle Representations in Quantum Field Theory, Lecture Notes in Physics 40, Springer 1996. 
[16] W. Kunhardt. On infravacua and superselection theory, J. Math. Phys. 39 (1998) 3589-3599 — hep-th/9704212.

[17] W. Kunhardt. On infravacua and the localization of sectors, J. Math. Phys. 39 (1998) 6353-6363 - math-ph/9806003.

[18] R. Wanzenberg. Energie und Präparierbarkeit von Zuständen in der lokalen Quantenfeldtheorie, Diploma thesis, Hamburg University (1987).

[19] D. Buchholz, S. Doplicher. Exotic Infrared Representations of Interacting Systems, Ann. Inst. H. Poincaré 40 (1984) 175-184.

[20] K. Fredenhagen, J. Hertel. Unpublished manuscript (1979).

[21] D. Buchholz, M. Porrmann. How Small is the Phase Space in Quantum Field Theory?, Ann. Inst. H. Poincaré 52 (1990) 237-257.

[22] D. Buchholz, P. Jacobi. On the Nuclearity Condition for Massless Fields, Lett. Math. Phys. 13 (1987) 313-323.

[23] F. Hars. Infravakua in der Quantenelektrodynamik, Diploma thesis, Hamburg University (1993).

[24] R.V. Kadison, J.R. Ringrose. Fundamentals of the Theory of Operator Algebras, Vol. 2: Advanced Theory, Academic Press 1986.

[25] J.E. Roberts. Lectures on Algebraic Quantum Field Theory, in "The Algebraic Theory of Superselection Sectors. Introduction and Recent Results", 1-112; ed.: D. Kastler, World Scientific 1989.

[26] D. Guido, R. Longo, J.E. Roberts, R. Verch. Charged Sectors, Spin and Statistics in Quantum Field Theory on Curved Spacetimes, Rev. Math. Phys. 13 (2001) 125-198 — math-ph/9906019.

[27] J.J. Bisognano, E.H. Wichmann. On the Duality Condition for Quantum Fields, J. Math. Phys. 17 (1976) 303-321.

[28] J.C. Baez, J. Dolan. From finite sets to Feynman diagrams, in "Mathematics Unlimited. 2001 and Beyond", vol. 1, 29-50; eds.: B. Engquist, W. Schmid, Springer 2001 — math.QA/0004133.

[29] S. Doplicher, J.E. Roberts. A new duality theory for compact groups, Invent. Math. 98 (1989) 157-218.

[30] S. Doplicher, J.E. Roberts. Why There is a Field Algebra with a Compact Gauge Group Describing the Superselection Structure in Particle Physics, Commun. Math. Phys. 131 (1990) 51-107.

[31] R.V. Kadison. The trace on finite operator algebras, Proc. Am. Math. Soc. 12 (1961) 973-977.

[32] S. Sakai. Operator algebras in dynamical systems, Encyclopedia of mathematics and its applications 41, Cambridge University Press 1991. 
[33] S. Doplicher, J.E. Roberts. Endomorphisms of $C^{*}$-algebras, cross products and duality for compact groups, Ann. Math. 130 (1989) 75-119.

[34] D. Buchholz, S. Doplicher, G. Morchio, J.E. Roberts, F. Strocchi. A Model for Charges of Electromagnetic Type, in "Operator Algebras and Quantum Field Theory", 647659; eds.: S. Doplicher et al., International Press, Cambridge 1997 — hep-th/9705089.

[35] D. Buchholz, S. Doplicher, G. Morchio, J.E. Roberts, F. Strocchi. Quantum Delocalization of the Electric Charge, Ann. Phys. 290 (2001) 53-66 — hep-th/0011015.

[36] L. Hörmander. The Analysis of Linear Partial Differential Operators I, Spriger 1990.

[37] R. Longo, J.E. Roberts. A Theory of Dimension, K-Theory 11 (1997) 103-159.

[38] P. Ghez, R. Lima, J.E. Roberts. $W^{*}$-categories, Pac. J. Math. 120 (1985) 79-109.

[39] S. McLane. Categories for the Working Mathematician, 2nd edition, Springer 1998.

[40] J.E. Roberts. The Statistical Dimension, Conjugation and the Jones Index, Rev. Math. Phys. 7 (1995) 631-643.

[41] N.N. Bogolubov, A.A. Logunov, A.T. Oksak, I.T. Todorov. General Principles of Quantum Field Theory, Kluwer 1990.

[42] J. Bros, H.Epstein, V.Glaser, R. Stora. Quelques aspects globaux des problèmes d'Edge-of-the-Wedge, in "Hyperfunctions and Theoretical Physics"; Lecture Notes in Mathematics 449, Springer 1975.

[43] D. Buchholz. Current Trends in Axiomatic Quantum Field Theory, in "Quantum Field Theory, Proceedings Tegernsee 1998"; eds.: P. Breitenloher, D. Maison, Lecture Notes in Physics 558, Springer 2000 - hep-th/9811233.

[44] D. Buchholz, R. Haag. The Quest for Understanding in Relativistic Quantum Physics, J. Math. Phys. 41 (2000) 3674-3697 — hep-th/9910243.

[45] D. Buchholz. Algebraic Quantum Field Theory: A Status Report - math-ph/0011044.

[46] A. Herdegen. Asymptotic Algebra for Charged Particles and Radiation, J. Math. Phys. 37 (1996) 100-120. 



\section{Acknowledgements}

I would like to express my deep gratitude to Prof. D. Buchholz for giving me the opportunity to work in this interesting area of mathematical physics, for his patient support, his constant interest in this work, and for many useful hints and pieces of advice.

I would also like to thank Prof. H. Roos for his kind willingness to co-supervise this thesis; his careful reading of the final manuscript lead to the elimination of several annoying misprints.

Finally, financial support from the Deutsche Forschungsgemeinschaft ("Graduiertenkolleg Theoretische Elementarteilchenphysik" at Hamburg University) during the years 1994-1997 is gratefully acknowledged. 



\section{Lebenslauf}

\section{Persönliche Daten:}

Name: $\quad$ Walter Kunhardt

Geburtsdatum: 13/09/1967

Geburtsort: Berchem-Ste--Agathe (Brüssel, Belgien)

Staatsangeh.: deutsch

\section{Schulbildung:}

09/73 - 07/85: Besuch der Europäischen Schule Brüssel II

08/07/1985: Abitur

\section{Studium:}

10/85 - 07/94: Studium der Physik an der Universität Hamburg

04/88: $\quad$ Vordiplom

07/94: Diplom; Diplomarbeit bei Prof. D. Buchholz:

"Über die universelle Algebra der lokalen Translationsoperatoren in der Algebraischen Quantenfeldtheorie"

08/94 - 09/97: Stipendiat des Graduiertenkollegs "Theoretische Elementarteilchenphysik" der Universität Hamburg; Beginn der Arbeit an der vorliegenden Dissertation.

seit 10/97: Wissenschaftlicher Mitarbeiter am Institut für Theoretische Physik der Universität Göttingen; Fertigstellung der Dissertation "On Infravacua and the Superselection Structure of Theories with Massless Particles", Betreuer: Prof. D. Buchholz. 